Portland State University

PDXScholar

Fall 12-9-2015

\title{
The Development of Personal Resources in the
} Academic Domain: Age Differences in the Evolution of Coping and Perceived Control and the Process Structures that Facilitate Academic Engagement

\author{
Teresa Marie Greene
}

Portland State University

Follow this and additional works at: https://pdxscholar.library.pdx.edu/open_access_etds

Part of the Psychology Commons

Let us know how access to this document benefits you.

\section{Recommended Citation}

Greene, Teresa Marie, "The Development of Personal Resources in the Academic Domain: Age Differences in the Evolution of Coping and Perceived Control and the Process Structures that Facilitate Academic Engagement" (2015). Dissertations and Theses. Paper 2632.

https://doi.org/10.15760/etd.2628

This Dissertation is brought to you for free and open access. It has been accepted for inclusion in Dissertations and Theses by an authorized administrator of PDXScholar. Please contact us if we can make this document more accessible: pdxscholar@pdx.edu. 
The Development of Personal Resources in the Academic Domain: Age Differences in the Evolution of Coping and Perceived Control and the Process Structures that Facilitate Academic Engagement

by

Teresa Marie Greene

A dissertation submitted in partial fulfillment of the requirements for the degree of

Doctor of Philosophy

in

Systems Science: Psychology

Dissertation Committee: Ellen A. Skinner, Chair

Wayne Wakeland

Thomas A. Kindermann

Pat Burk

Portland State University

2015 
(C) 2015 Teresa Marie Greene 
Abstract i

\begin{abstract}
Studies investigating the development of perceived control and coping in the academic domain generally adopt an individual differences approach, reporting meanlevel changes in these and associated constructs. Very few studies attempt to chart the process by which these personal resources exert individual and combined influences on academic outcomes, such as motivation and achievement, in light of normative developmental changes. Further, a consideration of reciprocal influences of these constructs on developmental changes and the contribution of social partners to these processes is not common.

Conceptualized from a systems perspective, this study integrates these different approaches in a longitudinal inquiry into the development of perceived control and coping, the impact of coping on academic engagement and achievement, and how support from the context shapes, and is subsequently shaped by, student behavior. An action-theoretic model is used to describe the hypothesized relationships, deriving from Deci \& Ryan's (1985) self-determination theory, and incorporating a flexible framework of coping as functionally similar yet structurally distinct strategies, defined as action-regulation under stress (Skinner, Edge, Altman, Sherwood, 2003; Skinner \& Zimmer-Gembeck, 2007). Four ways of coping are examined, divided into two categories: mastery coping, comprising problem-solving and information-seeking, and helplessness coping, comprising escape and confusion ways of coping. Contextual support is conceptualized as teacher provision of
\end{abstract}


Abstract ii

structure, involvement, and autonomy support. Engagement, as a motivational resource that leads to increased achievement, comprises both behavioral and emotional aspects of engagement. A tri-partite formulation of perceived control is used (Skinner, Chapman, \& Baltes, 1988a), comprising means-end (strategy), agency (capacity), and generalized control beliefs.

Data collected during one year of a four-year longitudinal study from 665 students in grades four and six, and fifty-three of their teachers, were used for this investigation. Normative developmental differences were examined through comparisons of mean-level shifts in each of the model constructs; regression -based analyses tested for age differences over time in the process structure of the model. Reciprocal influences of coping and engagement on teacher support and perceived control, and of engagement and achievement on coping, were also tested for age differences.

Results highlight the normative developmental changes that occur in these constructs during middle childhood, and indicate that the pattern of these changes is largely consistent with expectations; however, the process structure of the model relating the constructs of interest was found to be stable over time, with only one significant age difference detected: the influence on mastery coping of means-end control beliefs for effort. All other relationships tested did not differ significantly as children get older. Discussion focuses on evidence provided by the results of age trends in the developmental processes believed to be the drivers of change in the study 


\begin{abstract}
iii
constructs. Implications for the study of coping, regulatory processes, and features of the educational context, as they relate to the development of children's coping and control resources, are explored, with suggestions for the direction future research in these areas might take.
\end{abstract}


Dedication iv

\section{DEDICATION}

To Jesus Christ, into whose arms I run, who has given me every good gift and wills each breath I take, and by whose grace I am able to endure.

To my beautiful children, Zack and Sarah - you are the brightest stars in my darkest nights. You are now incredible, amazing adults and your light continues to shine out as a beacon to others. You are the reason I began this journey, and the strength that has sustained me through to the end.

To Irene Marshall and Anne Moore, the teachers of my youth who believed I could do and be anything I wanted to, and encouraged me to never give up.

And to the memory of my dear father, my friend, my confidante, my unfailing rock. who was so excited and incredibly proud that one of his kids was actually going to go this far with her education, and who couldn't wait to see the day she would walk the stage to accept her degree. I know he'll be watching me from above.

James Richard Kemp

$1939-2014$

May he rest in peace in the eternal light of heaven's glory. 


\section{ACKNOWLEDGMENTS}

I would like to offer my heartfelt thanks to the members of my dissertation committee for the support and insights they've provided to this project. To Ellen Skinner, my committee chair, mentor, advocate and source of inspiration, I can never adequately express my deep, deep gratitude for your support and caring compassion, or my true admiration of your endless brilliance. I have learned so much from you and no doubt will continue to long beyond this achievement. Thank you for never giving up on me. To Thomas Kindermann, thank you for your quiet and gentle way of pointing me in a better direction, and for your always positive support and encouragement. Your advice has always been spot on and just what was needed. To Wayne Wakeland, thank you for the many, many, inspiring and thought-provoking conversations about systems, and for always, always, always being upbeat and positive. You have truly been a bright spot in the landscape of my education. To Pat Burk, my friend and mentor in both academic and professional pursuits, I appreciate so much your amazing insights and knowledge. You have added a deep and enriching element to my thought process, and I look forward to many more generative conversations.

My deepest thanks goes to my family - my incredible husband, Greg, whose love, support, and patience has been epic. Your sacrifices over these long years have not gone unnoticed or unappreciated. You are an amazing friend and companion, and I am blessed that you are in my life. Thank you for all you do and are. 
To my parents, Jim and Dorene Kemp, for always being so embarrassingly proud of me, and for always pushing me to be the best I could be, even when I didn't want to be or couldn't see the value of such a thing! Without your belief in me and constant support, I never could have done this. Thanks for being my mom and dad!

To my children, who put up with all the hours of study, time spent being bored at the University while I did "stuff," and the ridiculous number of paper stacks that continued to multiply exponentially throughout our home, I thank you for your patience and excitement at mom doing something big. The emergence of your own love and pursuit of learning has been ample reward.

A big thank you to my friends, travel partners, and comrades in scholarship, Linda Newton-Curtis, Carrie Furrer, Gwen Marchand, and Tatiana Snyder: Your friendship, encouragement, warm memories, and your individual brilliance have made the journey so much more enjoyable. You are all such outstanding people and I am blessed to have known you.

I'd also like to acknowledge that data used in this study were collected through the support of the W.T. Grant Foundation, from Research Grant No. HD19914 from the National Institute of Child Health and Human Development, and from Training Grant No. 527594 from the National Institutes of Mental Health. I offer my most sincere thanks to the administrators, teachers, parents, and students of the Brockport School District for their gracious participation in that project. 


\section{TABLE OF CONTENTS}

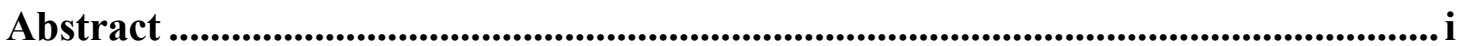

Dedication ................................................................................................................... iv

Acknowledgments......................................................................................................... v

List of Tables.......................................................................................................... xiv

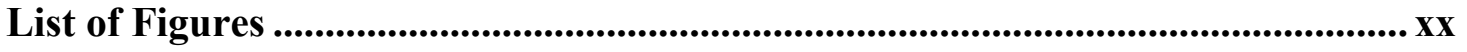

Chapter 1: Introduction ....................................................................................... 1

Perceived Control........................................................................................... 2

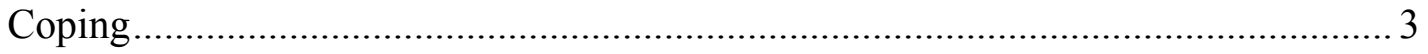

Personal Resources and Motivation...................................................................... 5

A Process-Oriented, Developmental Perspective .................................................... 6

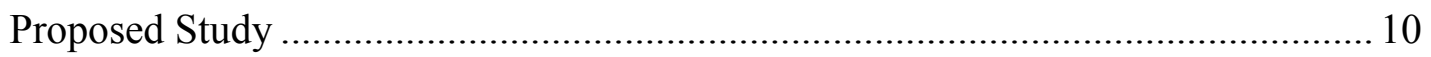

Chapter 2: Literature Review ................................................................... 13

Literature Review: Part 1 ....................................................................... 13

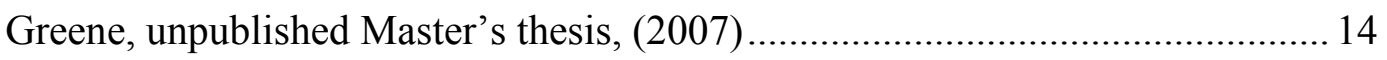

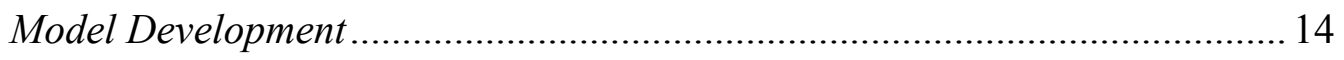

Conceptualization and Justification of Constructs .................................... 17

Study Goals and Hypothesized Model .......................................................... 30

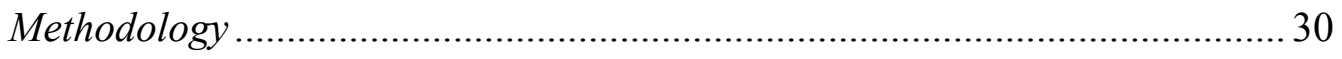

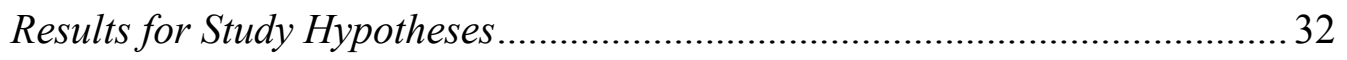




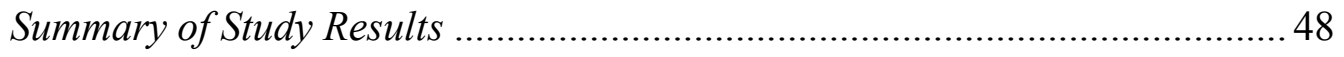

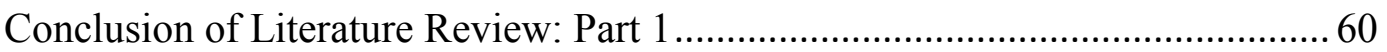

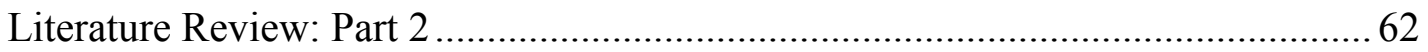

Normative Age Differences in Changes in Perceived Control, Coping, and

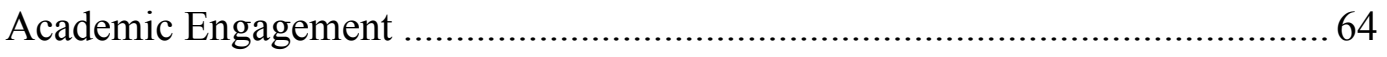

Normative Development of Perceived Control .......................................... 64

Skinner, Zimmer-Gembeck, and Connell, 1998...................................... 64

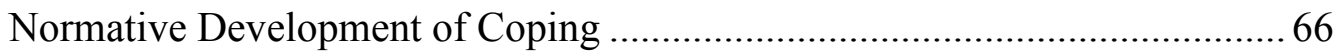

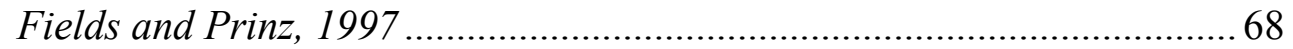

Compas, Connor-Smith, Saltzman, Thomsen, and Wadsworth, 2001 ........ 70

Skinner and Zimmer-Gembeck, 2007 ................................................. 71

Zimmer-Gembeck and Skinner, 2011 ............................................... 75

Normative Development of Engagement ................................................. 84

Fredericks, Blumenfeld, and Paris, 2004 ........................................... 84

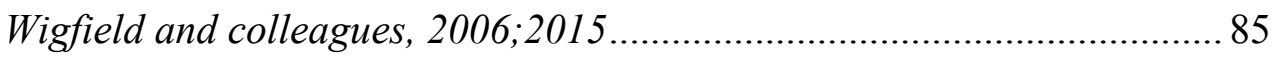

Summary of Normative Development of Target Constructs ........................ 86

Changes in Process Links Between Perceived Control, Coping, and Academic

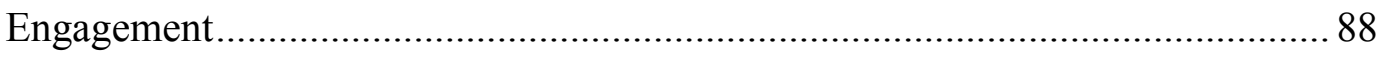

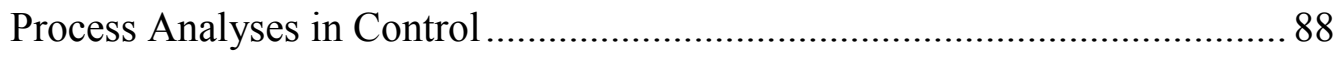




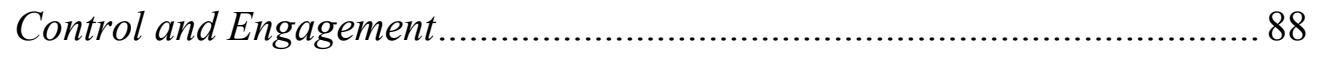

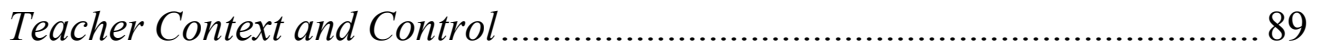

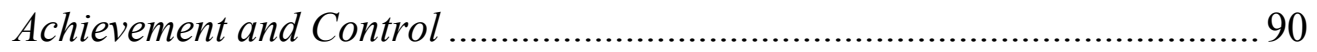

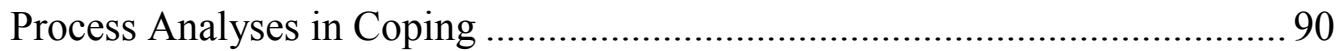

Skinner and Zimmer-Gembeck, 2011 .................................................... 91

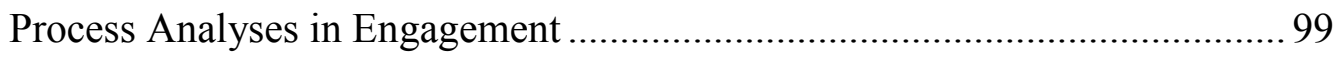

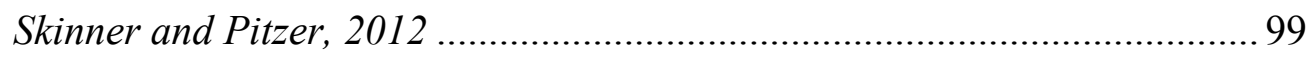

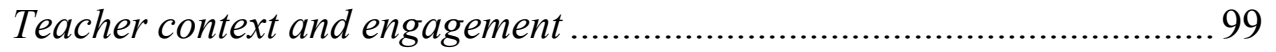

Coping and engagement ............................................................ 100

Reciprocal influences .................................................................. 101

Summary of Process Links Research .................................................. 102

Conclusions from the Literature Review: Part 2 2........................................... 104

Chapter 3: Purpose of Study ..................................................................................... 106

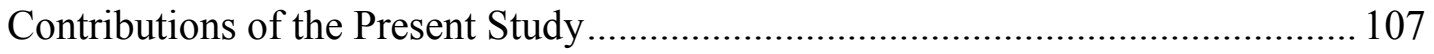

Research Question 1: Coping, Engagement, and Achievement ............................ 111

Coping Predicting Engagement............................................................. 112

Coping Predicting Changes in Engagement................................................ 113

Engagement Predicting Coping ......................................................... 114 


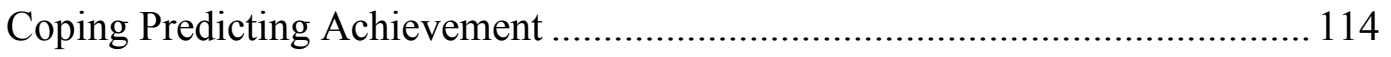

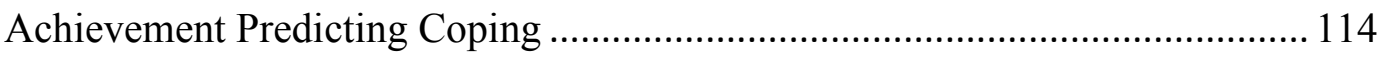

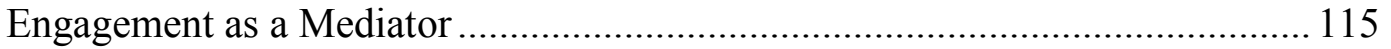

Research Question 2: Coping and Control ........................................................... 116

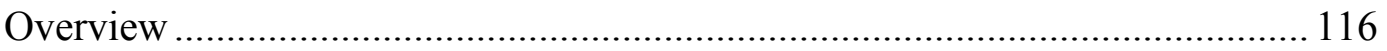

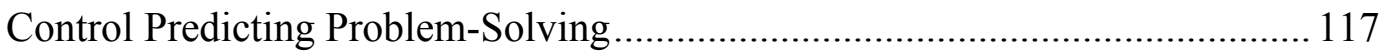

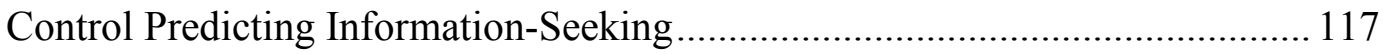

Control Predicting Escape ............................................................................ 118

Control Predicting Confusion ...................................................................... 119

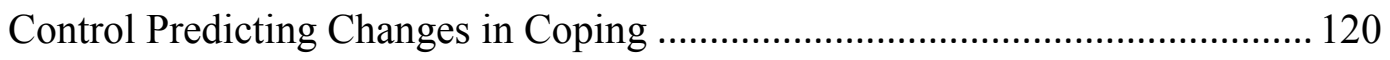

Research Question 3: Contextual Support and Coping ......................................... 122

Teacher Support Related to Coping, Concurrently ............................................. 122

Teacher Support Predicting Changes in Coping .............................................. 124

Research Question 4: Feedback Effects of Coping and Engagement ..................... 124

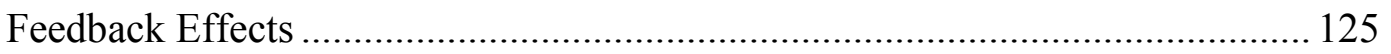

Chapter 4: Methods................................................................................................................ 131

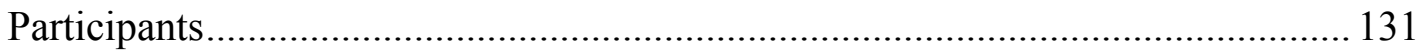

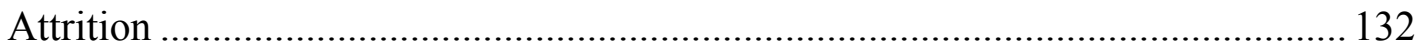




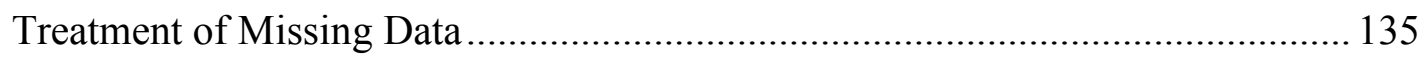

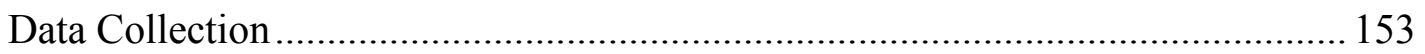

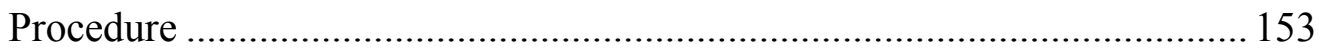

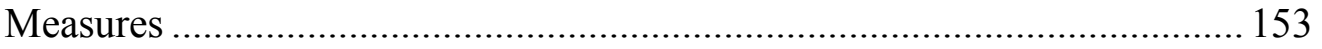

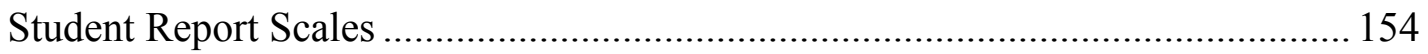

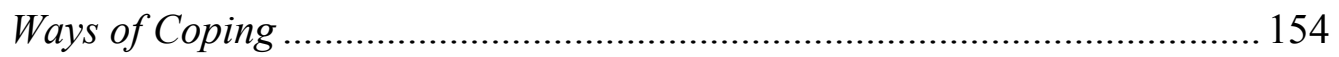

Self-System Measures: Perceived Control ................................................. 155

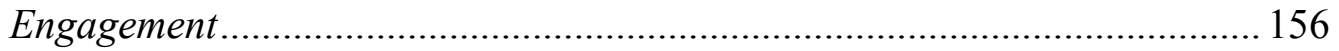

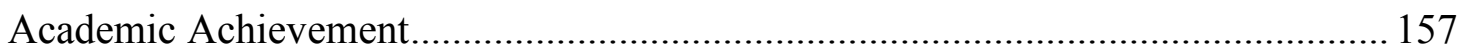

Teacher Motivational Support........................................................................... 157

Teacher Report of Provision of Structure ………………............................ 157

Teacher Report of Involvement ………………………............................ 158

Teacher Report of Provision of Autonomy Support ...................................... 159

Chapter 5: Results ................................................................................................................ 159

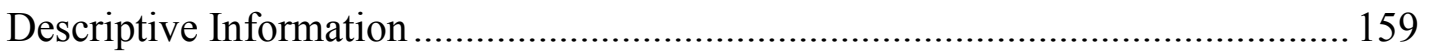

Scale Properties and Descriptive Statistics .................................................... 159

Interpretation of Means and Mean-level Differences ......................................... 162

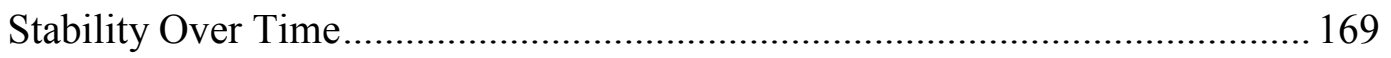

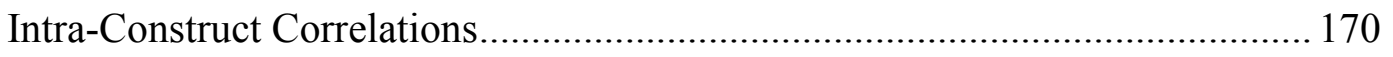




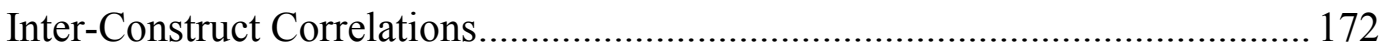

Assessment of Potential Multicollinearity .................................................... 174

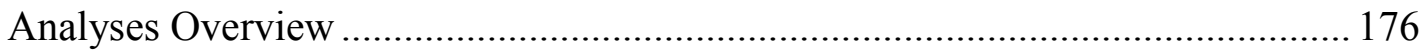

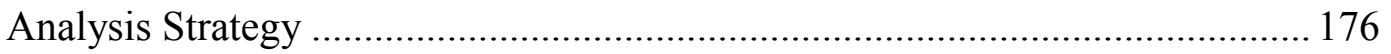

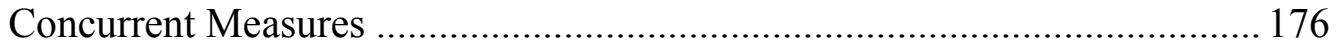

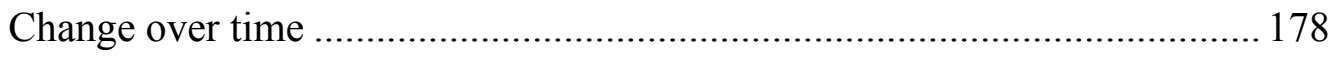

Mediational Analyses................................................................... 180

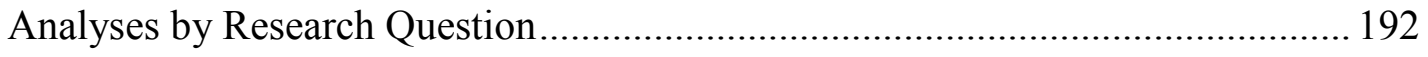

Research Question 1: Coping, Engagement, and Achievement ....................... 192

Coping Predicting Engagement......................................................... 195

Coping Predicting Changes in Engagement ............................................. 195

Feedback Effects - Engagement Predicting Coping................................... 196

Correlations - Coping and Achievement................................................ 197

Coping Predicting Achievement ............................................................... 198

Feedback Effects - Achievement Predicting Coping ................................. 199

Engagement as a Mediator .................................................................. 200

Research Question 2: Coping and Control ................................................... 206

Components of Control Related to Coping, Concurrently ........................... 207 
Control Aggregates Predicting Changes in Coping Over Time 212

Research Question 3: Contextual Support and Coping ................................... 215

Teacher Support Related to Coping, Concurrently ................................... 216

Teacher Support Predicting Changes in Coping .......................................... 218

Research Question 4: Feedback Effects of Coping and Engagement ............... 219

Feedback Effects of Coping on Control................................................... 220

Feedback Effects of Engagement on Control........................................ 221

Feedback Effects of Coping on Total Teacher Support ............................. 222

Feedback Effects of Engagement on Total Teacher Support...................... 222

Chapter 6: Discussion............................................................................. 281

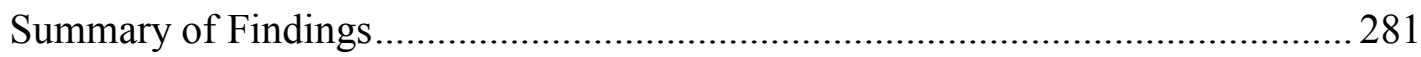

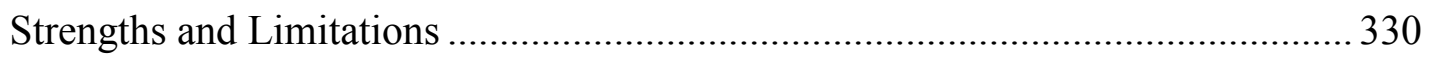

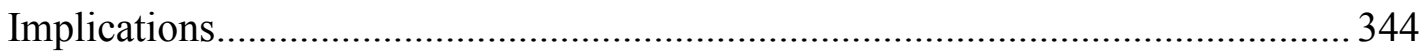

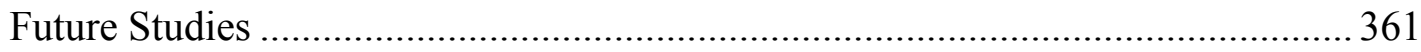

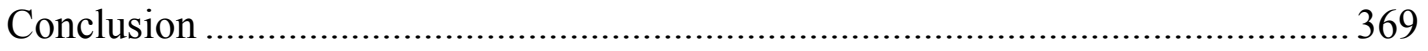

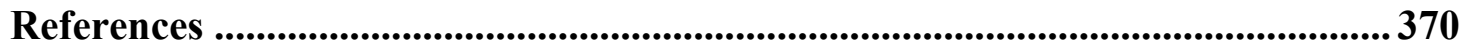

Appendix A: Questionnaire Items and Item Numbers ................................... 389

Appendix B: Formulas for Computing Aggregate Control Constructs ............... 396

Appendix C: Results Graphs........................................................................................... 398 
List of Tables xiv

\section{LIST OF TABLES}

Table 1: Summary of Results for All Confirmed Hypotheses for Greene (2007) ..34-38

Table 2: Summary of hypothesized results for Research Question 1 115

Table 3: Summary of hypothesized results for Research Question 2 121

Table 4: Summary of hypothesized results for Research Question 3 124

Table 5: Summary of hypothesized results for Research Question 4 127

Table 6: Summary of Hypotheses $128-130$

Table 7: Number of Students Assessed at Each Grade Level at Each Measurement Point for Year 2 132

Table 8: Properties of Measurement Scales, Descriptive Statistics, and Stabilities Full Sample $224-225$

Table 9: Properties of Measurement Scales, Descriptive Statistics, and Stabilities $4^{\text {th }}$ Grade Sample $226-227$

Table 10: Properties of Measurement Scales, Descriptive Statistics, and Stabilities $6^{\text {th }}$ Grade Sample $228-229$

Table 11: Intra-construct Correlations: Components of Perceived Control - Full

Sample 230 
Table 12: Intra-construct Correlations: Components of Perceived Control $-4^{\text {th }}$ Grade Sample 231

Table 13: Intra-construct Correlations: Components of Perceived Control $-6^{\text {th }}$ Grade

Sample 232

Table 14: Intra-construct Correlations: Components of Engagement, Coping, and Teacher Support - Full Sample. 233

Table 15: Intra-construct Correlations: Components of Engagement, Coping, and Teacher Support $-4^{\text {th }}$ Grade Sample

Table 16: Intra-construct Correlations: Components of Engagement, Coping, and Teacher Support $-6^{\text {th }}$ Grade Sample 235

Table 17: Inter-construct Correlations - Full Sample 236

Table 18: Inter-construct Correlations $-4^{\text {th }}$ Grade Sample .................................. 237

Table 19: Inter-construct Correlations $-6^{\text {th }}$ Grade Sample ................................. 238

Table 20: Age Differences in Construct Means by Time of Measurement ........239-240

Table 21: Age Differences in Fall-to-Spring Construct Correlations $241-246$

Table 22: Age Differences in Amount of Change from Fall-to-Spring in Construct Means 247

Table 23: Age Differences in the Influence of Ways of Coping on Engagement (Spring) 
Table 24: Age Differences in the Contribution of Ways of Coping to Changes in Engagement from Fall-to-Spring - Mastery Coping 249

Table 25: Age Differences in the Contribution of Ways of Coping to Changes in Engagement from Fall-to-Spring - Helplessness Coping 250

Table 26: Age Differences in the Feedback Effects of Engagement on Ways of Coping 251

Table 27: Age Differences in the Influence of Ways of Coping on Achievement ..... 252

Table 28: Age Differences in the Feedback Effects of Achievement on Ways of Coping 253

Table 29: First and Second Stage Mediation Paths - Engagement as a Mediator of the Effects of Coping on Achievement: Problem-Solving (Fall) 254

Table 30: First and Second Stage Mediation Paths - Engagement as a Mediator of the Effects of Coping on Achievement: Problem-Solving (Spring) 255

Table 31: First and Second Stage Mediation Paths - Engagement as a Mediator of the Effects of Coping on Achievement: Information-Seeking (Fall) 256

Table 32: First and Second Stage Mediation Paths - Engagement as a Mediator of the Effects of Coping on Achievement: Information-Seeking (Spring) .... 257

Table 33: First and Second Stage Mediation Paths - Engagement as a Mediator of the Effects of Coping on Achievement: Escape (Fall) 258

Table 34: First and Second Stage Mediation Paths - Engagement as a Mediator of the Effects of Coping on Achievement: Escape (Spring). 259 
Table 35: First and Second Stage Mediation Paths - Engagement as a Mediator of the Effects of Coping on Achievement: Confusion (Fall) 260

Table 36: First and Second Stage Mediation Paths - Engagement as a Mediator of the Effects of Coping on Achievement: Confusion (Spring)..... 261

Table 37: Significance Test of the Indirect Effect of Coping on Achievement, as Mediated Through Engagement 262

Table 38: Moderation of the Indirect Effect of Coping on Achievement, as Mediated Through Engagement 263

Table 39: Control Aggregates Predicting Ways of Coping - Mastery Coping 264

Table 40: Control Aggregates Predicting Ways of Coping - Helplessness Coping . 265

Table 41: Control Components (Strategy) Predicting Ways of Coping - ProblemSolving (Fall) 266

Table 42: Control Components (Strategy) Predicting Ways of Coping - InformationSeeking (Fall) 267

Table 43: Age Differences in the Contribution of Maximum Control to Changes in Coping from Fall-to-Spring - Mastery Coping ..... 268

Table 44: Age Differences in the Contribution of Maximum Control to Changes in Coping from Fall-to-Spring - Helplessness Coping 269

Table 45: Age Differences in the Contribution of Teacher Support to Ways of Coping - Mastery Coping 270 
List of Tables xviii

Table 46: Age Differences in the Contribution of Teacher Support to Ways of Coping

- Helplessness Coping.

271

Table 47: Age Differences in the Contribution of Teacher Support to Changes in

Coping from Fall-to-Spring - Mastery Coping

Table 48: Age Differences in the Contribution of Teacher Support to Changes in

Coping from Fall-to-Spring - Helplessness Coping

Table 49: Age Differences in the Feedback Effects of Ways of Coping on Changes in Total Strategy Beliefs - Mastery Coping 274

Table 50: Age Differences in the Feedback Effects of Ways of Coping on Changes in Total Strategy Beliefs - Helplessness Coping..... 275

Table 51: Age Differences in the Feedback Effects of Ways of Coping on Changes in Total Capacity Beliefs - Mastery Coping 276

Table 52: Age Differences in the Feedback Effects of Ways of Coping on Changes in Total Capacity Beliefs - Helplessness Coping..... 277

Table 53: Age Differences in the Feedback Effects of Ways of Coping on Changes in Total Teacher Support-Mastery Coping 278

Table 54: Age Differences in the Feedback Effects of Ways of Coping on Changes in

Total Teacher Support-Helplessness Coping 279

Table 55: Age Differences in the Feedback Effects of Engagement on Changes in Perceived Control and Total Teacher Support 280 
Table 56: Significant Differences in the Amount of Change in Fall to Spring Mean Scores for Strategy Beliefs.................................................................... 288

Table 57: Significance Differences in the Amount of Change in Fall to Spring Mean Scores for Coping Measures 293

Table 58: Significant Differences in the Amount of Change in Fall to Spring Mean Scores for Engagement

Table 59: Significant Differences in the Amount of Change in Fall to Spring Mean Scores for Teacher Support 296

Table 60: Structural Relationships and Developmental Trends for Research Question 1 304

Table 61: Structural Relationships and Developmental Trends for Research Question 2 . 308

Table 62: Structural Relationships and Developmental Trends for Research Question 3.

Table 63: Structural Relationships and Developmental Trends for Research Question 4 ..... 320 


\section{LIST OF FIGURES}

Figure 1: General Process Model....................................................................... 7

Figure 2: Self-Systems Model of Motivational Development (SSMMD),

(Connell, 1990, Connell \& Wellborn, 1991) ......................................... 15

Figure 3: The SSMMD with coping inserted into the action sequence. .................... 18

Figure 4: Full process model hypothesized for Master's thesis (2007); dotted lines indicate potential feedback pathways .................................................. 31

Figure 5: Final process model for Problem-Solving coping (Greene, 2007).............. 51

Figure 6: Final process model for Information-Seeking coping (Greene, 2007) ........ 54

Figure 7: Final process model for Escape coping (Greene, 2007) .......................... 57

Figure 8: Final process model for Confusion coping (Greene, 2007) ...................... 59

Figure 9: General process model that informs the formulation of research questions for the present study. Solid lines represent feedforward effects, dotted lines represent feedback effects

Figure 10: Causal loop diagram depicting dynamic feedforward and feedback relationships between ways of coping and engagement 112

Figure 11: Causal loop diagram depicting dynamic feedback effects of ways of coping and engagement on Teacher Support and Perceived Control.

Figure 12: Model for analyses to test age differences in the relationship between concurrent measures 
Figure 13: Model for analyses to test age differences in the prediction of change over time

Figure 14: Four steps to establish a meditational relationship (Baron \& Kenny, 1986)

Figure 15: Path model for simple mediation 185

Figure 16: Path model for a simple moderator model 186

Figure 17: Path model for multi-group interaction model depicting interaction terms for both the IV and the moderator; adapted from Preacher, Rucker, and Hayes, 2007 188

Figure 18: Specific pathways of the overall model addressed by Research Question 1 192

Figure 19: Grade * Coping interaction graphs for mastery ways of coping in the fall predicting spring Engagement when Achievement is in the model

Figure 20: Specific pathways of the overall model addressed by Research Question 2 206

Figure 21: Grade * Control interaction graphs for Total Strategy Beliefs in the fall predicting mastery ways of coping, concurrently. 210

Figure 22: Specific pathways of the overall model addressed by Research Question 3 215

Figure 23: Specific pathways of the overall model (bolded) addressed by Research Question 4 
Figure 24: Capacity Beliefs comparison graph - between and within grade differences. 286

Figure 25: Strategy Beliefs comparison graph - between and within grade differences..... 287

Figure 26: Generalized control beliefs comparison - between and within grade differences.....

Figure 27: Ways of Coping comparison graph - between and within grade differences.....

Figure 28: Engagement comparison graph -- between and within grade differences 293

Figure 29: Teacher Support comparison graph - between and within grade differences..... 295

Figure 30: Coping predicting Engagement; $6^{\text {th }}$ graders doing better than $4^{\text {th }}$ on PS \& $I S$. 305

Figure 31: Coping predicting changes in Engagement; $4^{\text {th }}$ graders doing more poorly on Escape. 305

Figure 32: Feedback effects of Engagement on Coping; $4^{\text {th }}$ graders doing better than $6^{\text {th }}$ overall 306

Figure 33: Total Strategy Beliefs predicting Coping; $6^{\text {th }}$ grade doing better than $4^{\text {th }}$ except on Escape 310

Figure 34: Strategy Belief components predicting Problem-Solving.... 312 
List of Figures $\quad$ xxiii

Figure 35: Strategy Belief components predicting Information-Seeking ................. 312

Figure 36: Teacher Support predicting ways of coping ....................................... 316

Figure 37: Teacher Support predicting changes in ways of coping. ....................... 316

Figure 38: Coping predicting changes in Total Strategy Beliefs; PS positively predicts

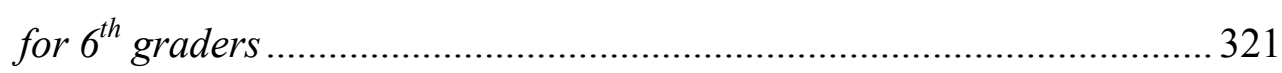




\section{CHAPTER 1: INTRODUCTION}

The personal resources a child brings to the classroom are instrumental to whether or not that child engages with academic tasks and is able to sustain engagement over time, particularly in the face of setbacks and challenges. For the purposes of the present study, personal resources are defined as internal processes, perceptions, repertoires of patterns of action, and other phenomena located within the individual student that are accessed and employed by the student in the service of achieving desired goals or avoiding undesired outcomes.

Research in the academic domain has demonstrated a strong and positive association between classroom engagement and academic achievement, and has identified many different student-level factors that exert positive and negative influences on engagement that can be conceptualized as personal resources (Fredericks, Blumenfeld, \& Paris, 2004; Wigfield \& Eccles, 2002; Wigfield, Eccles, Schiefele, Roeser, \& Davis-Kean, 2006; Wigfield, Eccles, Fredericks, Simpkins, Roeser, \& Shiefele, 2015). Two such influences that have been investigated as personal resources and found to be influential in promoting engagement and achievement are perceived control and coping strategies (Compas, 1987; Greene, 2007; Wigfield \& Eccles, 2002; Wolchik \& Sandler, 1997). A solid understanding of the processes by which these personal resources develop and the factors that influence that development is important for parents, teachers, and other adults who play a significant role in the lives of children, all of whom are in a position to shape 
children's academic experience and foster an intrinsic love of and motivation for learning.

Perceived Control. A long tradition of research in the area of control has yielded a wealth of information about different conceptualizations of control, with an understanding of control processes evolving through increasingly more complex and integrated conceptualizations. Beginning with studies regarding locus of control (Barling, 1982; Greene, 1985; Prawat, 1976; Prawat, Grissom, \& Parish, 1979), the control field moved through the development of attribution theory (Ames \& Archer, 1988; Andrews \& Debus, 1978; Pomerantz \& Saxon, 2001; Stipek \& Mason, 1987), understanding of the learned helplessness phenomenon (Butkowsky \& Willows, 1980; Chapin \& Dyck, 1976; Craske, 1985; Dweck, 1975; Dweck \& Reppuci, 1973; Fowler \& Peterson, 1981; Medway \& Venino, 1982), of perceived competence (Boggiano, Main \& Katz, 1988; Bouffard, Boisvert, \& Vezeau, 2003; MacIver, Stipek, \& Daniels, 1991; Phillips, 1984; Spinath \& Spinath, 2005; Wong, Weist, \& Cusick, 2002) and self-efficacy (Bong, 2001; Bouffard, Boileau, \& Vezeau, 2003; Bouffard-Bouchard, Parent, \& Laravee, 1991), and currently focuses on the construct of perceived control (Lopez, 1999; Patrick, Skinner, \& Connell, 1993; Skinner, Chapman, \& Baltes, 1988a; Skinner, Wellborn, \& Connell, 1990; Skinner, Zimmer-Gembeck, \& Connell, 1998; Stipek \& Weisz, 1981; for an in-depth discussion of the diversity among control constructs, see Skinner, 1996; for a chronological review of control research in the academic domain, see Greene, 2007). 
In the academic domain, the self-system process most salient for the student is the competence system, with the multiple aspects of perceived control the most often used measures of the strength and functioning of that self-system (Connell, 1990; Connell \& Wellborn, 1991). Perceived control is a tri-partite construct formulated from an action-theoretic perspective that integrates the primary features of the preceding control constructs (Skinner, et al., 1988a). A considerable body of research has presented convincing evidence supporting the assertion that perceived control as a construct is positively related to gains in academic achievement (Findley \& Cooper, 1983; Kalachstein \& Nowicki, 1997; Nowicki \& Strickland, 1973; Nolen-Hoksema, Girgus, \& Seligman, 1986; Sink, Barnett, \& Hixon, 1991). Furthermore, research relating specific components of control with a student's ability to engage with challenging academic tasks, or re-engage productively when faced with a failure or setbacks, is becoming more prevalent (Skinner, et al., 1998).

Coping. The field of coping has made repeated attempts to sort out the complex questions of conceptualization, classification, and measurement, with little progress made over its relatively short history. A promising framework has been suggested by Skinner and her colleagues (Skinner, Edge, Altman, \& Sherwood, 2003) that categorizes ways of coping into "families" of functionally similar, but structurally different strategies. This conceptualization allows for the possibility that coping strategies may be a resource that develops over time to maintain the functional 
outcome of the coping effort, but also allows for behavioral manifestations that are age-graded and developmentally appropriate.

For example, the activities of observation and researching a topic are both coping strategies that may equally accomplish the outcome of exercising control over one's context by gathering information about that context. In the framework proposed by Skinner et al. (2003), these strategies are classified into a family called information-seeking, yet one response may be more appropriate to a small child, while the other is a more appropriate response for an adult. Both adults and children engage in periods of observing the world around them, such as in the use of social referencing to gauge what an appropriate action or response to a particular situation may be. However, children use this strategy far more frequently than adults, who are more apt to use instrumental means of gathering information, such as utilizing technology to access and sort through large amounts of information. By the same token, one certainly would not expect to see a toddler "Googling" on his computer, as this is a method most commonly used by adults to search the vast wealth of information available through the Internet. Having noted this age-related distinction in strategy use, both responses can still be discussed under the same organizing principle of “information-seeking."

With respect to the competence system, Skinner et al. (2003) identified four families of coping responses that serve as markers of this system: problem-solving, information-seeking, escape, and confusion. Examples of problem-solving include 
strategizing, self-talk, self-encouragement, and hypothesis-testing, in addition to direct problem-solving. The information-seeking family includes responses such as monitoring, planning, and strategies for building a knowledge base regarding contingencies in a given situation. Escape coping includes responses that assist a person to withdraw from, avoid, or put off an undesirable event and include activities such as daydreaming, physical withdrawal, overt and covert avoidance tactics, pessimism, procrastination, and self-handicapping. Examples of confusion coping include helplessness, discouragement, panic, and self-doubt.

Personal resources and motivation. Engagement as a motivational construct is multi-faceted in nature (Fredericks, et al., 2004; Skinner, Furrer, Marchand, \& Kindermann, 2008; Skinner, Kindermann, \& Furrer, 2009; Skinner \& Pitzer, 2012). Skinner and Wellborn (1994), in a motivational model positing the fulfillment of three fundamental psychological needs as critical to the initiation and maintenance of motivated behavior, conceptualized classroom engagement as academic motivation, comprising two complementary components, behavior and emotion. Behavioral engagement addresses the extent to which a student exerts effort, persists at a task or problem, and is attentive to the situation. Emotional engagement is evidenced through the display or experience of emotions such as worry, anger, happiness, boredom, or sadness when faced with academic tasks. As a marker for the competence system, perceived control is posited in this model to support engagement when control is internal, and success is attributable to controllable causes, whether internal or external, 
and failure to internal, controllable causes. Perceived control is thought to be counterproductive to motivated behavior when control is perceived to be primarily external, and success is attributable to unknown or uncontrollable causes. The effects of perceived control on engagement are mediated by the coping strategies activated in the student by the input received from the context as well as experiences of effectiveness that either build up or deplete his or her internal resources.

A process-oriented, developmental perspective. A considerable amount of the research in the fields of control and coping has focused on identifying different facets of each, their antecedents, consequences, and synergistic effects (Cain \& Dweck, 1995; Causey \& Dubow, 1992; Diener \& Dweck, 1978; 1980; Mantzicopoulos, 1990; 1997; Obach, 2003; Skinner \& Wellborn, 1991, as cited in Skinner, 1992; Urdan, Midgley, \& Anderman, 1998). Many of these studies have invoked process models to frame the investigation of these relationships. In general, these models are based on theories that posit ways in which perceived control influences children's actions in the classroom, including their engagement with school tasks and how they cope with difficulties and failures. In turn, engagement and coping are thought to shape children's actual performance, as indicated by their subject grades and standardized test scores (Bouffard \& Vezeau, 1998; Greene, Miller, Crowson, Duke, \& Akey, 2004; Midgley \& Urdan, 1995; Miller, Greene, Montalvo, Ravindran, \& Nichols, 1996; Pokay \& Blumenfeld, 1990; Wolters, 2004) Other process models explicate theories about the social antecedents of perceived control, including involvement, structure, 
and autonomy support, provided by teachers and parents in the classroom and home environments (Connell, Spencer \& Aber, 1994; Marchand \& Skinner, 2007;

Oettingen, Little, Lindenberger, \& Baltes, 1994; Schneewind, 1995).

One such general process model underlying the more specific models tested in previous research consistent with these theories is presented in Figure 1. Elaboration of the components of this model to more specific constructs has generated studies that support the process links described by this model, identifying the specific features of each construct that activates the next step in the process.

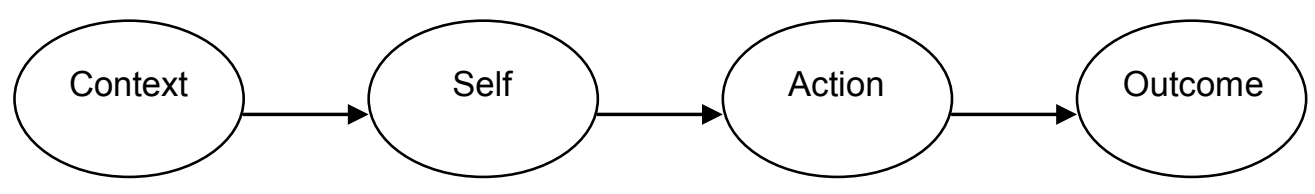

Figure 1: General process model.

For example, the specific components of teachers, parents, and peers have been investigated as features of the social context that shape the self-systems of the individual (Connell, 1990; Furrer \& Skinner, 2003; Marchand \& Skinner, 2007). Likewise, the specific components of perceived control have been examined as features of the self most closely linked to the motivated action of engagement with school tasks (Skinner et al., 1998). It is this type of investigation and specification that has led to the particular conceptualizations of the main constructs of interest to this study, namely, the differentiated view of perceived control, the hierarchical 
structure of the families of ways of coping, and the multi-faceted nature of engagement as a motivational construct.

Given this observation, this body of research as a whole can be critiqued on three major points. First, many of the studies that claim to be process-oriented focus on the relationships described by concurrent correlations, which do not directly examine the basic tenet of a process approach, namely, how one construct influences changes over time in another construct. Correlations, whether concurrent, partial, or semi-partial, are a necessary step in confirming process links, and are consistent with the proposed direction of a particular link. However, they are also consistent with the proposition that links may function in the opposite direction, as correlations do not prove causation, but rather, imply an association. For example, most studies investigating the relationship between control and engagement find a correlation between the two constructs and interpret it as control exerting an influence on engagement; however, it could also be interpreted as engagement having an influence on control, as some few studies have shown (e.g., Walls \& Little, 2005).

Experimental and intervention studies have provided support for the causal effects of the context on perceived control (Craske, 1985; Dweck, 1975; Dweck \& Reppucci, 1973), thus confirming the existence of a relationship in the proposed direction between these constructs. However, studies conducted in a naturalistic setting, such as the classroom, can provide additional support for the process by which these variables influence one another by examining the effects of one variable on 
changes in its hypothesized consequence over time, as in the case of academic progress from the beginning of the school year to the end. This is the approach taken by the current study.

Second, the dynamics of process described by feedforward and feedback effects may create cycles that are the basis for changes in mean levels of key constructs, as are noted in conjunction with changes in age, suggesting possible developmental trends. Whenever children of different ages are included in a study, mean level differences between ages (or grades, as a proxy for age) should always be examined to identify developmental differences in constructs so these cyclical processes can be detected and explored further. In this way, a study can make a valuable contribution to the explanation of the mechanisms by which the constructs of interest exert their influence on development, providing useful insights for future research. This approach is also incorporated in the present study.

Third, the idea of developmental shifts leads to the consideration of the possibility that age-related changes are not just limited to the mean levels of the constructs, but that the process structure itself may also be subject to change. As a child matures, it is possible that the process links themselves operate in different ways. For example, the social context may have a stronger influence on coping when children are young, whereas self-system processes may play a bigger role as children enter middle school. Most process models assume that the processes are stable over 
any developmental changes in the mean levels of the variables. This assumption is directly examined in the present study.

Present study. In a study conducted for this researcher's unpublished Master's thesis (Greene, 2007), a model derived from the general process model depicted in Figure 1 was tested. This study investigated the strength and direction of the links between supportive actions of classroom teachers (context), perceived control (self), coping (action), and student engagement and achievement (outcomes). Four different models were obtained, one for each of four ways of coping; two ways of coping believed to promote positive outcomes (problem-solving and information-seeking) referred to as "mastery-oriented" coping, and two ways believed to frustrate them (confusion and escape), referred to as "helplessness" coping. The present study continues the investigation of these models with an examination of how the personal resources of perceived control and coping develop over time, and how the process structure of the models previously obtained changes with that development.

Specifically, the present study addresses two primary goals. The first goal is to examine mean-level age differences in the constructs of interest, both concurrently and over time as normative development progresses. The second goal of the study is to identify developmental differences in the process structure of the models previously obtained in this researcher's thesis work by testing for the moderating effect of grade on the robustness of the direct and mediational relationships between the constructs, as well as the reciprocal feedback and feedforward cycles. Pursuit of this line of inquiry 
could yield valuable information regarding the inter-individual differences and intraindividual changes of students with respect to the personal resources important to academic endeavors, and how those differences impact the way motivational processes unfold over time to promote or undermine subsequent academic outcomes.

The literature review supporting these goals unfolds in two parts within Chapter 2. In the first part of the chapter, an extensive review of this researcher's unpublished thesis study and subsequent findings is provided, including a presentation of the four models obtained. The hypotheses of the present study were informed by and extend the prior study.

The second part of the chapter presents a review of the research conducted to examine developmental differences in the constructs relevant to the present study; this part proceeds in two sub-sections. The first sub-section describes studies that have sought to shed light on the normative progression of development of each of the three constructs of interest individually. The second sub-section discusses the few studies that have undertaken the task of examining the developmental changes in how these constructs relate to each other over time, taking into consideration the simultaneous developmental changes within the constructs themselves.

A description of the objectives of this study is presented in Chapter 3, and includes a more detailed explanation of the research questions, hypotheses, and how this investigation contributes to an understanding of the developmental progression of the personal resources of perceived control and coping. The study methodology, 
measures, and procedures used for data collection and preparation of the data for analysis are presented in Chapter 4, and Chapter 5 presents the technical details and results for each analysis conducted to explore the data and test the study hypotheses. The sixth and final chapter summarizes the study foundations, goals, hypotheses, and results, and concludes with a discussion of its strengths, limitations, implications, and how future research formulated from a systems perspective can continue this line of inquiry in a fruitful manner. 


\section{CHAPTER 2: LITERATURE REVIEW}

The literature review for the present study is divided into two parts. The first part presents an in-depth review of this researcher's unpublished Master's thesis study that provides the conceptualization of constructs and analytic groundwork for the present study. The second part continues the review by exploring the current literature in the specific areas of interest to the present study: the normative development of perceived control and coping, and the developmental changes that occur over time in the relationships among these and the other relevant constructs. Together these two parts will offer a solid foundation upon which the present study may proceed. The first part of this review explaining this researcher's previous work, which provides the starting point for the formulation of the current study's hypotheses, begins here.

Literature Review: Part 1.

Very few studies to date have sought to integrate distinct programs of research in the areas of coping, perceived control, and academic motivation in such a way as to facilitate a process-oriented investigation of how the combined influences of personal resources may operate concurrently and over time to promote or frustrate engagement in the classroom. Presented here in the first part of this literature review is a detailed summary of one such study that defines the launching point for the goals of the present investigation. The results of this study are essential to the formulation of the hypotheses of the present study regarding developmental outcomes. 
Greene, unpublished Master's thesis, 2007. In a study conducted for this researchers's Master's thesis, which was designed to examine the individual and combined contributions of the personal resources of coping and perceived control to children's academic motivation in the classroom, a model was constructed and tested that was derived from the motivational framework depicted in Figure 2. The study examined concurrent, feedforward, and feedback effects among these constructs: perceived control, contextual support (conceptualized as different forms of support from the teacher), ways of coping (mastery-oriented ways and helplessness ways), academic motivation (conceptualized as engagement), and academic achievement. A discussion of the development of the model that was tested is provided next, followed by an explanation of the conceptualization of the constructs depicted in the model, which have been adopted for the present study. The review continues with an account of the specific findings obtained in this study, summarized in Table 1 in that section, and a discussion of the four different models that resulted.

\section{Model Development}

As used in previous studies (Furrer \& Skinner, 2003; Marchand \& Skinner, 2006), this researcher's unpublished thesis study utilized the Self-Systems Motivational Model of Development (SSMMD) as an integrative framework for constructing a specific model of relationships to be tested (Connell, 1990; Connell \& Wellborn, 1991; Skinner \& Wellborn, 1994). This model is an action-theoretic model derived from a motivational model conceived earlier by researchers in the area of self- 
determination theory, which is predicated on the assumption of three basic psychological needs: relatedness, competence, and autonomy (Deci \& Ryan, 1985). These three needs are integral components of the self-system, the satisfactions of which are assumed to be critical to the initiation and maintenance of motivated behavior. Figure 2 presents the SSMMD, illustrating how researchers expanded on the more general model presented in Figure 1 in the Introduction of the present study.

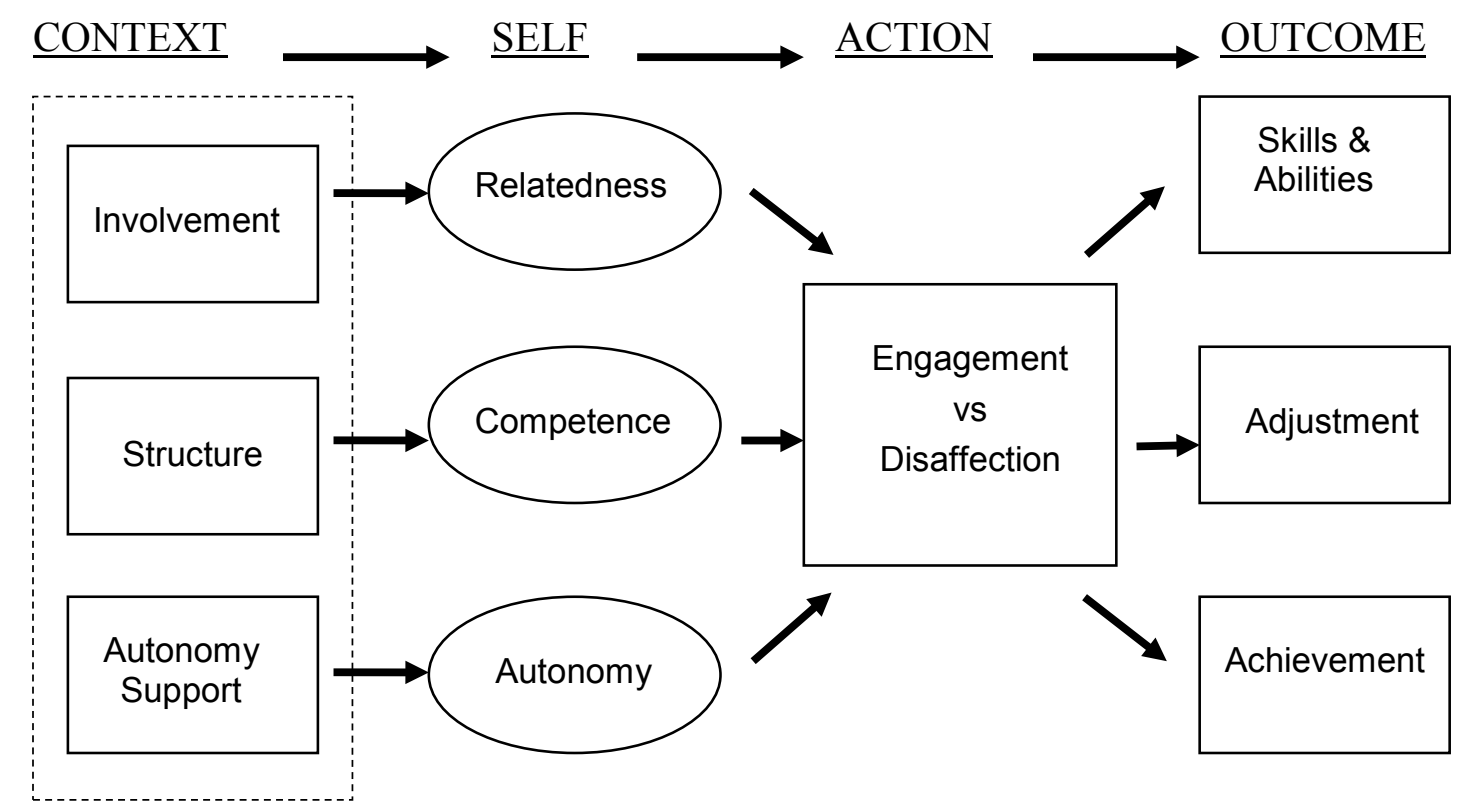

Figure 2: Self-Systems Model of Motivational Development (SSMMD) (Connell, 1990; Connell \& Wellborn, 1991).

Integration of the constructs of interest. This model provides a unique approach for examining each of the constructs of interest to this study and offers a theoretical basis for hypothesizing about their potential relationships. Characteristics of the social context are posited to influence the fulfillment of three fundamental 
psychological needs through fostering children's self-system perceptions and building capacity within these important personal resources. More specifically, when the context provides structure, the competence system is strengthened. When the context offers warmth and involvement, relatedness is fostered, and when the context promotes a variety of choices that support the emergence of a person's authentic self, the autonomy system is bolstered.

In the model depicted in Figure 2, increased capacity in the self-systems is posited to facilitate increases in motivation and subsequently more positive outcomes. Engagement is the motivational construct of interest in both this researcher's unpublished Master's thesis study and the present study, which is conceptualized as having both behavioral and emotional components. This understanding of engagement as a multi-faceted construct is consistent with the action-theoretic basis of the SSMMD and is particularly useful in examining coping as a mechanism through which facilitation of motivation can occur. The action-theoretic perspective suggests that children's responses to challenges in the classroom can be broken down into the categories of emotion, behavior, and attention (orientation), thereby providing a basis for correspondence between the components of engagement and the components of coping as action-regulation. Given this underlying theoretic base, the SSMMD provides a convenient structure for incorporating coping into the model.

The working definition of coping adopted by the present study defines coping as "an organizational construct that describes how people regulate their own behavior, 
emotion, and motivational orientation under conditions of psychological distress" (Skinner \& Wellborn, 1994, p. 112). By adopting this definition, the potential for inclusion of coping, as a regulatory, personal resource that acts as the gateway for students to connect with the behavioral and emotional components of motivation under the distress of academic challenges and setbacks, can be realized.

The theoretical basis of the SSMMD further suggests that regulation of behavior, emotion, and orientation is activated through an appraisal process whereby resource levels of the self-systems are assessed. As the self-system most closely associated with the academic domain, the competence system is assessed through appraisals of the resource of perceived control. This appraisal process is hypothesized to be the force that moves a child into action, or as the models under investigation in this researcher's unpublished Master's thesis study posit, into the selection and implementation of particular coping strategies. In this way the SSMMD provides an excellent framework for investigating the process linkages between these constructs that may facilitate engagement and achievement in the face of academic demands.

Figure 3 illustrates how the model accommodates the inclusion of coping as part of the action sequence with engagement.

\section{Conceptualization and justification of constructs}

The conceptualization for each of the four main constructs in this researcher's unpublished Master's thesis study was based on previous research exploring, examining, testing, and reviewing each. A brief overview of the theoretical basis for 
each construct and supporting investigations, where reviewed in the thesis study, are given here.

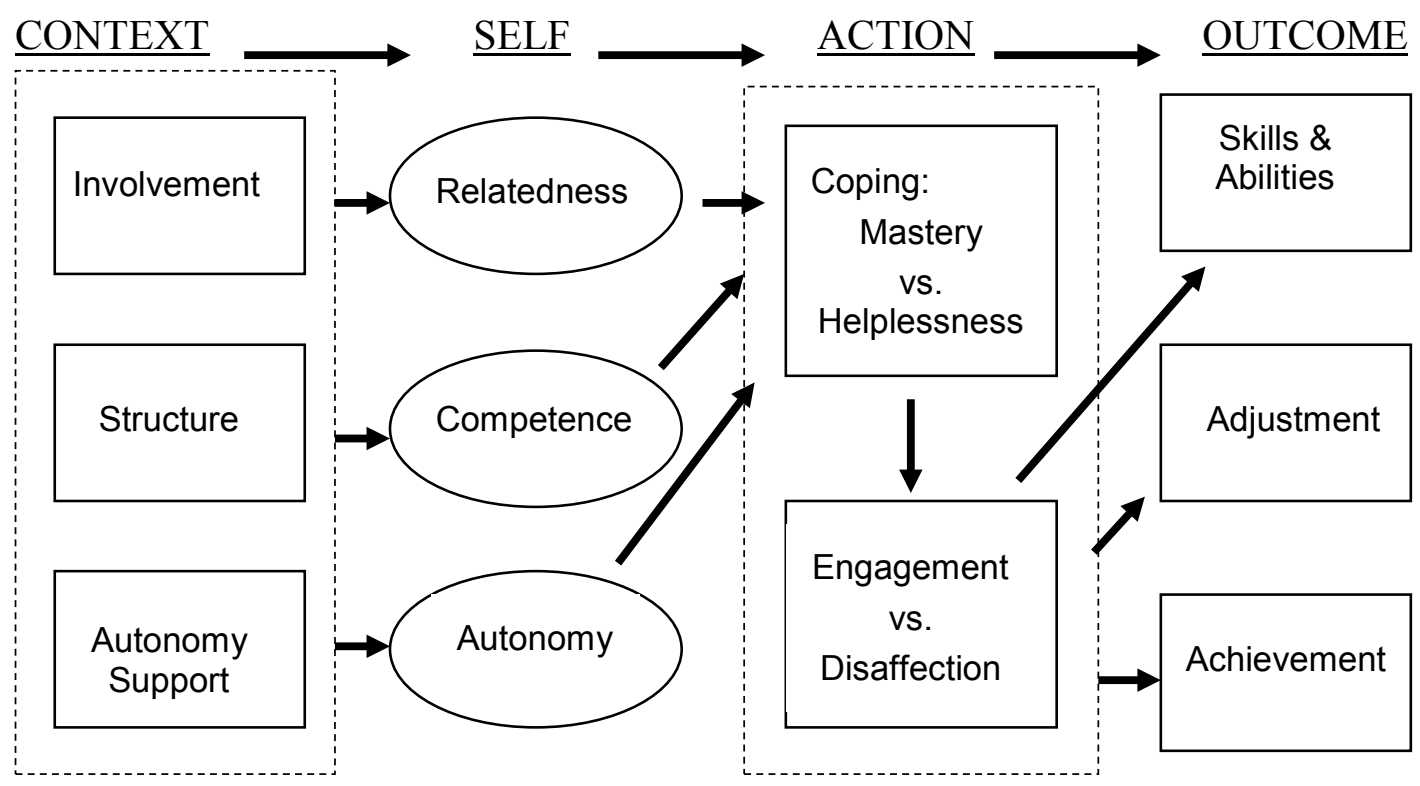

Figure 3: The SSMMD with coping inserted into the action sequence.

Perceived control. A robust and decades long history of research in the control field, particularly in the academic domain, has yielded a rich literature chronicling the development of understanding of the various facets of control. After several emergent constructs had risen, been tested, and given way to new constructs, a final conceptualization was advanced that has now held the notable position of the latest conceptualization of control for over two decades. This conceptualization of control - perceived control—is tripartite in structure, and combines facets of its predecessors into one construct that has proven exceptionally valuable in applications involving processes in the academic domain. 
Formulated from an action-theoretic perspective, wherein action, defined as goal-directed intentional behavior, is the unit of analysis, rather than responses, Skinner and her colleagues (1988a), proposed that a person can construct sets of beliefs about the relationships between the functional components embodied by all the other control constructs, namely agents, means, and ends, and that these belief sets are instrumental in guiding subsequent action and influencing performance outcomes. These three sets of beliefs, when taken together, form the multi-faceted construct of perceived control.

Control beliefs are generalized expectancies about the extent to which a person can produce desired outcomes and avoid undesired ones, and are functionally equivalent to perceived competence. Agency beliefs refer to beliefs a person has regarding whether he or she has access to specific means for achieving desired outcomes and avoiding undesired ones, and is similar to self-efficacy. Means-ends beliefs are beliefs about what means lead to specific outcomes, and is most analogous to locus of control. For clarity, these belief sets are more commonly referred to as generalized control beliefs, capacity beliefs, and strategy beliefs, respectively.

These belief sets can work together synergistically to produce action and influence subsequent outcomes, particularly through the interaction of capacity and strategy beliefs. Strategy beliefs comprise 5 beliefs (ability, effort, powerful others, luck, and unknown causes) and are like locus of control in that they refer to the location of the contingent reinforcement. The internal-external distinction is preserved 
with ability and effort on the internal end, and powerful others, luck, and unknown causes on the external end. The experience of a successful outcome becomes the contingent reinforcement for employing the most effective strategy for success, which either arises from within the person (internal location), or is located in a source external to the individual. Capacity beliefs are like self-efficacy beliefs in that they refer to an assessment made by the person about the access they have to 4 specific means (strategies) for effecting successful outcomes: ability, effort, powerful others, and luck.

Strategy and capacity beliefs can also work independently from one another in that each operates in reference to persons in general, while the preceding control constructs were person and context specific. This means that a person's generalized control beliefs are not held to the rules of logic; one can believe he or she has a high degree of control in any given situation without stopping to consider the means required for success, or whether he or she has access to those means. Likewise, one can believe certain means will lead to a desired outcome, yet have no confidence in his or her ability to enact the necessary strategy. Conversely, one may believe he or she has access to a variety of means while at the same time believe none of those means will be effective in achieving the desired outcome. In this way, the distinction among the three facets of the construct is preserved and analytic confounding of their influences can be avoided. 
In the literature review for this researcher's unpublished Master's thesis study, six previous studies that had examined some aspect of this construct were summarized. Four of these studies were conducted by Skinner and her colleagues, three of which were summarized in total. The fourth study was a large, extensive inquiry resulting in the publication of a monograph, and only a portion of this study was discussed.

In the first of these studies, Skinner and her colleagues tested and confirmed the validity and reliability of an instrument to measure all three sets of beliefs (Skinner, Chapman, \& Baltes, 1988a). Their methodology included testing against established measures of other control constructs to confirm hypothesized similarities and distinctions between the subscales of the new conceptualization and the preceding constructs. All hypotheses were supported as expected.

The next study in this set explored the influence of perceived control on academic motivation, its mediational role between contextual support and engagement in the classroom, and possible reciprocal effects (Skinner, Wellborn, \& Connell, 1990). Findings supported not only the hypothesis that the distinct facets of perceived control contribute differentially to engagement, consistent with previous research involving other constructs of control, but also hypotheses regarding its relationship with contextual support. Additional analyses tested the interaction of strategy and capacity beliefs for effects on engagement. Results showed that ability and effort contribute in distinct ways to a student's level of engagement, specifically suggesting 
that efficacy judgments are most important with respect to the effects of beliefs about effort on engagement, while controllability is the most influential dimension of ability beliefs. Testing of the full path model yielded support for reciprocal effects among model components.

The third study conducted by Skinner and her colleagues incorporated the selfsystem process of autonomy into the model to test for the differential effects of perceived control and autonomy on engagement (Patrick, Skinner, \& Connell, 1993). Both constructs predicted engagement, and both contributed uniquely to the variance in engagement. No interaction effects between them were found. Additional analyses using the interaction scores of strategy and capacity beliefs to predict engagement yielded confirming evidence for the findings of previous research testing these same relationships.

In a landmark study, Skinner, Zimmer-Gembeck, and Connell (1998) tested this conceptualization of perceived control and its effects on academic motivation in a longitudinal study using aggregate measures of perceived control to represent different types of control profiles. The items for perceived control were aggregated to yield two composite scores representing the extremes of positive and negative control. A third aggregation was created from these two to represent the most powerful combination of beliefs thought to maximally promote control. Analyses using these three constructs yielded results indicating that for all age groups those control profiles wherein the child held positive expectancies for success, believed that certain strategies would lead 
to achievement of desired goals, and that he or she could access those strategies were positively and significantly related to engagement. Conversely, the control profile wherein the child indicated her or she did not know what strategies would lead to the achievement of desired outcomes, or else believed that certain strategies would lead to success and that he or she did not have access to those strategies were negatively and significantly related to engagement.

Analyses using the components of perceived control yielded results suggesting that when children feel they have access to a variety of strategies, or that effort will lead to positive outcomes, they are more likely to remain engaged in the classroom in the face of challenging tasks and events. Conversely, when children don't know what strategies lead to success, or don't believe they have access to the strategies that are effective in achieving desired goals, they are more likely to disengage with their classroom tasks, or may not even try to engage at all.

Other programs of research have used this construct in different motivational models and obtained complementary results. Lopez (1999) conducted a study that incorporated goal orientations as a motivational process that may be facilitated by perceived control. Only capacity beliefs and control expectancies were included from the perceived control construct. Ability Capacity Beliefs were found to be positively related to a mastery goal orientation and negatively related to a performance goal orientation. Control expectancies were not related to either. 
Walls and Little (2005) used the constructs for Effort and Ability Capacity Beliefs in a mediational analysis to test whether these components of perceived control would act as mediators of the effects of self-regulated motivation on achievement. Effort Capacity Beliefs were found to be mediators between motivation and achievement, but Ability Capacity Beliefs were not. This study provided a unique perspective in that it tested the effects of motivation on control, rather than control on motivation, and offered some evidence in support of further investigation of the reciprocal effects of motivation and control.

Coping. Researchers in the field of coping have long been challenged by the lack of consensus about a central classification system for the myriad ways of coping that have been tested and explored over the years. In a survey of over 100 different assessments used to measure ways of coping with different types of stressors, more than 400 different "ways" were noted (Skinner, Edge, Altman, \& Sherwood, 2003). To address this problem and provide a consistent framework for conceptualizing and measuring coping, these authors suggested the concept of "families" of coping that would serve to group ways of coping together into families of functionally similar responses.

This framework is based on an action-theoretic definition of coping as action regulation under stress. This definition implies coping is a goal-directed, organized response to stressful transactions with the environment, comprising components of desire, behavior, emotion, and orientation (Skinner \& Wellborn, 1997). Desire 
represents the goal objective, with behavior, emotion, and orientation as the subjects of regulation. In order to achieve an adaptive outcome from a stressful transaction with the environment, these elements need to be managed in a coordinated fashion. All three objects of regulation represent distinct, yet related, ways of coping, which, when taken together, form the construct of "action" as conceptualized by action theories.

In this researcher's unpublished Master's thesis study, as well as in the present study, the competence system is the self-system of interest, and control is the target of desire, or the goal objective. Behaviors are oriented towards detecting contingencies in the environment that facilitate experiences of control, emotions are persistent and determined, and the orientation is focused on learning how to produce desired outcomes and avoid undesired ones. Coping serves an adaptive function in the competence system in facilitating the necessary coordination of these responses and the individual's actions with the contingencies available in the environment. In the framework of coping families devised by Skinner and her colleagues (2003), the overarching ways of coping that organize the components of coping as action into families of functionally homogenous responses are characterized as either adaptive or maladaptive. These terms, adaptive and maladaptive, translate into mastery-oriented or helplessness-oriented in relation to the competence system. In this framework, the families most salient to the competence system are problemsolving and information-seeking (adaptive, or mastery-oriented), and escape and 
confusion (maladaptive, or helplessness-oriented). Other "ways" of coping that belong to these families appear throughout the literature, and several studies for each family were reviewed. Four studies were reviewed for problem-solving that included strategizing, problem-solving and hypothesis testing; five studies for informationseeking were reviewed and included the activities of monitoring, planning, and information-seeking; three studies were reviewed for escape that included procrastination, denial, and lack of effort; for the confusion family, which includes helplessness, discouragement, panic, and self-doubt, only one study was reviewed, which focused on the antecedents and consequences of helplessness.

The review of these studies noted that for responses associated with the two mastery-oriented families of ways of coping (problem-solving and informationseeking), positive relationships were found with perceptions of control, levels of academic engagement and student achievement. Children with high perceptions of control were more active and successful in their problem-solving efforts, and sought information about their tasks and ways to complete them more frequently. In turn, they were also more deeply engaged with their work, and enjoyed higher levels of achievement in the classroom, suggesting a mediating role for coping between control and academic outcomes.

For the responses associated with the helplessness families of ways of coping (escape and confusion), just the opposite was true. Children with low control tended to deal with more feelings of self-doubt, negative affect, helplessness, and a tendency 
to avoid challenge. In turn, negative relationships were demonstrated for the helplessness ways of coping with levels of academic engagement and student achievement.

Engagement. Over the past several years, the construct of engagement has been of increasing interest to researchers in the area of motivation, particularly that of academic motivation (Wigfield et al., 2006; Wigfield et al., 2015). While there is consensus regarding the multi-dimensionality of the construct, and even about what types of responses those dimensions encompass (behavioral, emotional, and cognitive), there is still considerable debate about what the specific indicators of each dimension are (Fredericks, Blumenfeld, \& Paris, 2004).

The conceptualization of engagement has been approached from a variety of theoretical traditions, depending on the domain of interest. In keeping with the underlying theoretical base of the SSMMD, engagement in this researcher's unpublished Master's thesis is conceptualized from an action-theoretic perspective, as a motivational force comprised of behavior, emotion, and orientation that is persistent, enthusiastic, and directed towards the goal of mastery of academic material.

Recent research has explored the dimensionality of the engagement construct and confirmed a multi-dimensional structure comprising two distinct types of dimensions. The first dimension distinguishes engagement from disaffection. Disaffection is not the absence of engagement, but rather an active state of being in its own right that describes a negative tenor of the behaviors, emotions, and orientations 
in play for the person at the time. Within each of these dimensions a further distinction can be made in that behavior can be distinguished from emotion. Therefore, the engagement construct can be broken down into behavioral engagement, behavioral disaffection, emotional engagement, and emotional disaffection. These dimensions can be combined in a variety of ways to characterize a student's engagement according to the specific questions being addressed (Skinner et.al., 2009).

This conceptualization of engagement is particularly attractive to educational researchers as it reflects the types of interactions believed to lead to actual learning, and subsequently, higher levels of achievement. Moreover, it is a concept that is potentially changeable, subject to influence by any number of social partners or intervention efforts. It also reflects the opposite of high quality interactions of this nature, which is easily recognizable as disaffection. This state is marked by apathy, withdrawal, and disinterest in the activities of learning (Connell \& Wellborn, 1991; Skinner, et al., 2008).

The engagement scales in this researcher's Master's thesis study were formulated so that the items for disaffection were subtracted from those for engagement, yielding two composite scores, one for behavior and one for emotion. These were then combined into one score to represent a student's level of overall engagement. The psychometric properties of this conceptualization have been tested in previous research and found to be satisfactory (Skinner \& Belmont, 1993; Furrer \& Skinner, 2003; Skinner et al., 2009). 
Contextual Support. The action-theoretic perspective assumes interaction between the person and his or her social context; the quality of these interactions is believed to influence the quality of subsequent engagement with challenges presented by the environment. As specified by the SSMMD, the support provided by the context can either build capacity or diminish it in a person's self-system perceptions. When the context provides warmth and acceptance, perceptions of relatedness are enhanced. When the context provides structure, perceptions of control are strengthened, and when the context provides choices that support a person's true sense of self, the perception of having the freedom to express oneself (autonomy), and the courage to do so, is fostered.

In the academic domain, specifically the classroom, the teacher is the primary social partner representing the context, providing these crucial supports to help students build their internal resources. This type of contextual support is believed to help activate a desire to pursue mastery of academic material, a desire often referred to as motivation, or in this conceptualization, high quality engagement.

Teacher support is measured as provision of involvement, structure, and autonomy support. The measures are used independently and as a combined construct to represent overall contextual support. Previous research has tested the measures used and found them to be adequate (Skinner \& Belmont, 1993). 
Study goals and hypothesized model

While the theoretical models that informed this researcher's unpublished Master's thesis depicted all three self-system process, the study was focused on the personal resource of perceived control, the widely accepted marker of the competence system; hence, only the competence system was incorporated in the hypothesized model to be tested. All forms of contextual support, regardless of which self-system they were targeted towards, were utilized to provide a fuller picture of the influences impacting the competence system.

The study goals were four-fold: 1) to examine the relationships among coping, engagement, and achievement, including the mediational role of engagement in the relationship between coping and achievement; 2) to investigate the direction and strength of the relationships between facets of perceived control and coping, examining whether and how they shape each other over time; 3) to explore the impact of contextual factors, such as the provision of various types of support from the child's teacher, on a child's exercise of coping resources; and 4) to explore feedback effects of children's coping and engagement on subsequent levels of their predictors (perceived control and contextual support). Each of these goals gave rise to a set of hypothesized relationships to be tested, represented by the model depicted in Figure 4 .

\section{Methodology}

Sample characteristics and data collection. Data for this study were collected in the course of a 4-year longitudinal study from students and their teachers in grades 
3 through 7 enrolled in a rural sub-urban school district in the northeast area of the United States. Administrative data were also collected in the form of standardized test scores for a subset of children in the sample. After data cleaning procedures the final data set used for analysis contained information for approximately 1000 students and 53 teachers. Self-report measures of perceived control, coping, and both behavioral and emotional engagement and disaffection were collected from the students through surveys administered during the course of a school day, in the fall of the year, and then again in the spring of the same academic year. Measures of perceived teacher support were also obtained from the students at both time points. Teachers were surveyed regarding their perceptions of their students' engagement behaviors, and their own provision of support, in both fall and spring.

\section{$\underline{\text { CONTEXT }} \underline{\text { SELF }} \underline{\text { ACTION }}$ OUTCOME}

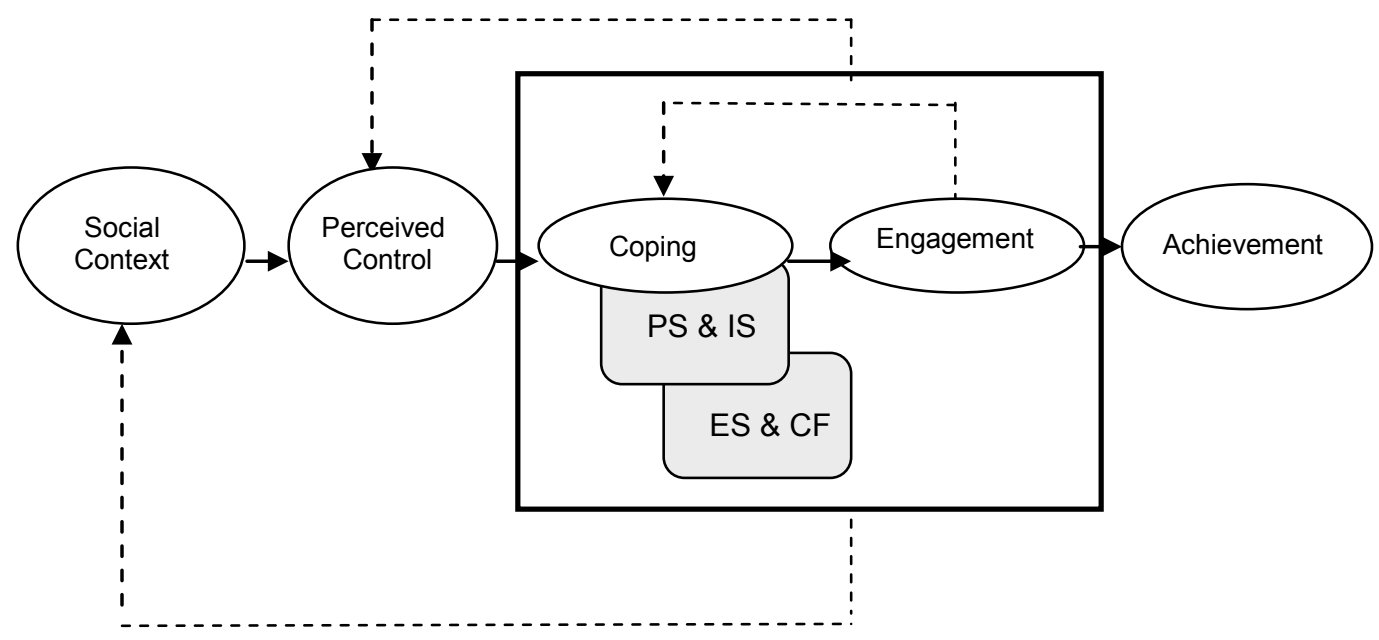

Figure 4: Full process model hypothesized for Master's thesis; dotted lines indicate potential feedback pathways. 
Preliminary analyses. Concurrent relationships among the constructs were examined, as well as construct stability over time. Of particular interest was an absence of a strong relationship between certain ways of coping and achievement. Only Information-Seeking in the spring, and Escape coping in the fall were related to achievement. All constructs were found to be sufficiently stable; somewhat of concern was the high stability of the combined Engagement construct from fall to spring. It was noted that high stability of constructs over time may interfere with the detection of changes in those constructs.

Results for Study Hypotheses

Multiple regression analyses were conducted to test each set of hypotheses, corresponding to the goals of the study and the pathways of the hypothesized model. The results of these analyses are summarized in Table 1, and discussed here, according to the study goals previously listed.

\section{Goal 1: Coping, Engagement, and Achievement}

This set of hypotheses aimed to determine the strength and direction of the relationships between these constructs, including the potential role of Engagement as a mediator between ways of coping and Achievement.

Correlations. Examination of relationships among these elements demonstrated a positive link between both ways of mastery coping and all engagement constructs, and a negative link between both ways of helpless coping and all engagement constructs, at both the fall and spring time points. Overall Engagement 
was positively related to Achievement; however, as previously noted, only Information-Seeking in the spring, and Escape in the fall showed a significant link with Achievement.

Coping predicting changes in Engagement. Using the overall Engagement construct as well as the individual component constructs (Behavioral Engagement and Emotional Engagement), and after controlling for levels of these constructs in the fall, hierarchical regression analyses supported a predictive relationship for the helpless ways of coping to changes in these constructs in the spring. Specifically, Escape negatively predicted Behavioral Engagement only, while Confusion negatively predicted overall Engagement. No predictive relationships were found for the mastery ways of coping to changes in any of the engagement constructs. These results suggest that while students who are prone to using ways of coping indicative of helplessness are more likely to have lower levels of subsequent classroom engagement, children who typically employ mastery-oriented ways of coping are already well engaged with their class work and are not likely to experience an increase in that engagement due to the coping strategies they use.

Reciprocal effects. Conversely, overall Engagement in the fall predicted changes in all ways of coping in the spring, after controlling for levels of ways of coping in the fall. This suggests that initial levels of classroom engagement are important to which ways of coping a student will employ to negotiate academic challenges and setbacks, with low levels of initial Engagement leading to subsequent 


\begin{tabular}{|c|c|c|c|c|c|c|c|c|c|}
\hline 1. & Correlations & & & & & & & & \\
\hline \multirow{2}{*}{1.1} & \multirow{2}{*}{ Perceived Control with Coping } & \multicolumn{2}{|c|}{ Problem-Solving } & \multicolumn{2}{|c|}{ Information-Seeking } & \multicolumn{2}{|c|}{ Escape } & \multicolumn{2}{|c|}{ Confusion } \\
\hline & & Fall & Spring & Fall & Spring & Fall & Spring & Fall & Spring \\
\hline \multirow[t]{11}{*}{$1.1 a$} & Control Components & & & & & & & & \\
\hline & Strategy: Effort & + & + & + & + & & - & + & + \\
\hline & Ability & & + & & - & + & + & + & + \\
\hline & Powerful Others & - & - & - & - & + & + & + & + \\
\hline & Luck & - & - & - & - & + & + & + & + \\
\hline & Unknown & - & - & - & - & + & + & + & + \\
\hline & Capacity: Effort & + & + & + & + & - & - & - & - \\
\hline & Ability & + & + & + & + & - & - & - & - \\
\hline & Powerful Others & + & + & + & + & - & - & - & - \\
\hline & Luck & + & + & + & + & - & - & - & - \\
\hline & Generalized Control Beliefs & + & + & + & + & - & - & - & - \\
\hline \multirow[t]{6}{*}{$1.1 b$} & Control Aggregates & & & & & & & & \\
\hline & Total Strategy & - & - & - & - & + & + & + & + \\
\hline & Total Capacity & + & + & + & + & - & - & - & - \\
\hline & Promote & + & + & + & + & - & - & - & - \\
\hline & Undermine & - & - & - & - & + & + & + & + \\
\hline & CONMAX & + & + & + & + & - & - & - & - \\
\hline
\end{tabular}




\begin{tabular}{|c|c|c|c|c|c|c|c|c|c|}
\hline \multicolumn{10}{|c|}{$\begin{array}{l}\text { Table } 1 \\
\text { Summary of Results for All Confirmed Hypotheses for Greene (2007), cont'd }\end{array}$} \\
\hline 1.0 & \multicolumn{9}{|l|}{ Correlations } \\
\hline \multirow[t]{6}{*}{$1.1 c$} & \multirow{2}{*}{$\begin{array}{l}\text { Control Interactions } \\
\text { (Strategy X Capacity) }\end{array}$} & \multicolumn{2}{|c|}{ Problem-Solving } & \multicolumn{2}{|c|}{ Information-Seeking } & \multicolumn{2}{|c|}{ Escape } & \multicolumn{2}{|c|}{ Confusion } \\
\hline & & Fall & Spring & Fall & Spring & Fall & Spring & Fal & Spring \\
\hline & Effort & + & + & + & + & - & - & + & + \\
\hline & Ability & + & + & - & - & + & + & + & + \\
\hline & Powerful Others & - & - & - & - & + & + & + & + \\
\hline & Luck & - & - & - & - & + & + & + & + \\
\hline \multirow{5}{*}{1.2} & Fno & \multicolumn{2}{|c|}{ Problem-Solving } & \multicolumn{2}{|c|}{ Information-Seeking } & \multicolumn{2}{|c|}{ Escape } & \multicolumn{2}{|c|}{ Confusion } \\
\hline & Engagement witn coping & Fall & Spring & Fall & Spring & Fall & Spring & $\mathrm{Fal}$ & Spring \\
\hline & Overall Engagement & + & + & + & + & - & - & - & - \\
\hline & Behavioral & + & + & + & + & - & - & - & - \\
\hline & Emotional & + & + & + & + & - & - & - & - \\
\hline \multirow{6}{*}{1.3} & \multirow{2}{*}{ Teacher Support with Coping } & \multicolumn{2}{|c|}{ Problem-Solving } & \multicolumn{2}{|c|}{ Information-Seeking } & \multicolumn{2}{|c|}{ Escape } & \multicolumn{2}{|c|}{ Confusion } \\
\hline & & Fall & Spring & Fall & Spring & Fall & Spring & Fal & Spring \\
\hline & Overall Teacher Support & + & + & + & + & - & - & - & - \\
\hline & Provision of Structure & & & + & & & & & \\
\hline & Provision of Involvement & + & + & + & + & - & - & - & - \\
\hline & Provision of Autonomy Support & + & + & + & + & - & - & - & - \\
\hline 1.4 & Achievement with Coping & & & & + & & - & & \\
\hline 1.5 & Engagement with Achievement & Ove & & & havioral & + & Em & & \\
\hline
\end{tabular}




\begin{tabular}{|c|c|c|c|c|c|c|c|c|c|c|c|}
\hline \multicolumn{12}{|c|}{$\begin{array}{l}\text { Table } 1 \\
\text { Summary of Results for All Confirmed Hypotheses for Greene (2007), cont'd }\end{array}$} \\
\hline 2.0 & \multirow{2}{*}{\multicolumn{2}{|c|}{$\begin{array}{l}\text { Unique Effects } \\
\text { Control }\end{array}$}} & \multicolumn{2}{|c|}{$\begin{array}{l}\text { Problem- } \\
\text { Solving }\end{array}$} & \multicolumn{2}{|c|}{$\begin{array}{l}\text { Information- } \\
\text { Seeking }\end{array}$} & \multicolumn{2}{|c|}{ Escape } & \multicolumn{2}{|c|}{ Confusion } & \multirow{2}{*}{ Comments } \\
\hline 2.1 & & & Fall & Spring & Fall & Spring & Fall & Spring & Fall & Spring & \\
\hline \multirow{5}{*}{$2.1 a$} & \multirow{5}{*}{$\begin{array}{l}\text { Control } \\
\text { Aggregates }\end{array}$} & Total Strategy & + & + & & & + & + & + & + & \multirow{2}{*}{$\begin{array}{l}\text { Despite being correlated, } \\
\text { each makes a unique } \\
\text { contribution to Prob-Solv. }\end{array}$} \\
\hline & & Total Capacity & + & + & + & + & - & - & - & - & \\
\hline & & Promote & + & + & + & + & - & - & & & Strength increases $\mathrm{w} /$ time. \\
\hline & & Undermine & & & - & - & - & - & + & + & Strongest for Escape. \\
\hline & & CONMAX & & & & & & & & & \\
\hline \multirow{5}{*}{$2.1 b$} & \multirow{5}{*}{$\begin{array}{l}\text { Strategy } \\
\text { Components }\end{array}$} & Effort & + & + & + & + & & & + & + & \multirow{6}{*}{$\begin{array}{l}\text { Contributors to } \\
\text { Information-Seeking } \\
\text { become more } \\
\text { differentiated and } \\
\text { influential with age; } \\
\text { external more influential } \\
\text { than internal. }\end{array}$} \\
\hline & & Ability & & & & - & & & + & + & \\
\hline & & Powerful Others & - & - & - & - & + & + & + & + & \\
\hline & & Luck & & & & & + & + & + & + & \\
\hline & & Unknown Causes & - & - & - & - & + & + & + & + & \\
\hline \multirow{4}{*}{$2.1 c$} & \multirow{4}{*}{$\begin{array}{l}\text { Capacity } \\
\text { Components }\end{array}$} & Effort & + & + & + & + & - & - & - & - & \\
\hline & & Ability & + & + & + & + & - & - & - & & \\
\hline & & Powerful Others & & & + & + & - & - & & & \\
\hline & & Luck & & & + & & - & - & - & - & \\
\hline \multirow{5}{*}{$2.1 d$} & \multirow{5}{*}{$\begin{array}{l}\text { Control } \\
\text { Interactions }\end{array}$} & Effort & + & + & + & + & - & - & + & + & \\
\hline & & Ability & & & + & + & - & - & - & - & \\
\hline & & Powerful Others & - & - & - & - & - & - & & & \\
\hline & & Luck & & & & & - & - & + & + & \\
\hline & & Unknown Causes & & & & & - & - & + & + & \\
\hline
\end{tabular}

Note: $+=$ positive correlation $;-=$ negative correlation; blank space $=$ no significant association 


\begin{tabular}{|c|c|c|c|c|c|c|c|c|c|c|c|}
\hline \multicolumn{12}{|c|}{$\begin{array}{l}\text { Table } 1 \\
\text { Summary of Results for All Confirmed Hypotheses for Greene (2007), cont'd }\end{array}$} \\
\hline \multirow[t]{2}{*}{2.2} & \multirow{2}{*}{\multicolumn{2}{|c|}{ Teacher Support }} & \multicolumn{2}{|c|}{$\begin{array}{l}\text { Problem- } \\
\text { Solving }\end{array}$} & \multicolumn{2}{|c|}{$\begin{array}{l}\text { Information- } \\
\text { Seeking }\end{array}$} & \multicolumn{2}{|c|}{ Escape } & \multicolumn{2}{|c|}{ Confusion } & \multirow[t]{2}{*}{ Comments } \\
\hline & & & Fall & Spring & Fall & Spring & Fall & Spr & Fall & Spring & \\
\hline \multirow{3}{*}{$2.2 a$} & \multirow{3}{*}{$\begin{array}{l}\text { Support } \\
\text { Components }\end{array}$} & Structure & & & & & & & & & \\
\hline & & Involvement & & & & & & & & & \\
\hline & & $\begin{array}{l}\text { Autonomy } \\
\text { Support }\end{array}$ & + & + & + & + & - & - & - & - & \\
\hline 3.0 & \multicolumn{2}{|c|}{ Predictors of Change } & \multicolumn{3}{|c|}{ Problem-Solving } & \multicolumn{3}{|c|}{ Information-Seeking } & \multicolumn{2}{|c|}{ Escape } & Confusion \\
\hline \multirow{8}{*}{3.1} & \multirow{8}{*}{$\begin{array}{l}\text { In coping, } \\
\text { from fall to } \\
\text { spring }\end{array}$} & Coping in the fall & \multicolumn{3}{|c|}{+} & \multicolumn{3}{|c|}{+} & \multicolumn{2}{|c|}{+} & + \\
\hline & & Total Strategy & \multicolumn{3}{|c|}{-} & \multicolumn{3}{|c|}{-} & \multicolumn{2}{|c|}{+} & + \\
\hline & & Total Canacitv & \multicolumn{3}{|c|}{+} & \multicolumn{3}{|c|}{+} & \multicolumn{2}{|c|}{-} & - \\
\hline & & Promote & \multicolumn{3}{|c|}{+} & \multicolumn{3}{|c|}{+} & \multicolumn{2}{|c|}{-} & - \\
\hline & & Undermine & \multicolumn{3}{|c|}{-} & \multicolumn{3}{|c|}{-} & \multicolumn{2}{|c|}{+} & + \\
\hline & & CONMAX & \multicolumn{3}{|c|}{+} & \multicolumn{3}{|c|}{+} & \multicolumn{2}{|c|}{-} & - \\
\hline & & Teacher Support & \multicolumn{3}{|c|}{+} & \multicolumn{3}{|c|}{+} & & 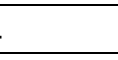 & \\
\hline & & Engagement & & + & & & + & & & . & - \\
\hline
\end{tabular}

Note: $+=$ positive correlation; $-=$ negative correlation; blank space = no significant association 


\begin{tabular}{|c|c|c|c|c|c|c|c|c|c|c|}
\hline \multicolumn{11}{|c|}{$\begin{array}{l}\text { Table } 1 \\
\text { Summary of Results for All Confirmed Hypotheses for Greene (2007), cont'd }\end{array}$} \\
\hline 3.0 & \multicolumn{2}{|c|}{ Predictors of Change } & \multirow{2}{*}{$\begin{array}{c}\text { Teacher } \\
\text { Support } \\
+\end{array}$} & \multirow{2}{*}{$\begin{array}{c}\text { Provision of } \\
\text { Involvement }\end{array}$} & \multirow{2}{*}{$\begin{array}{c}\text { Provision of } \\
\text { Autonomy } \\
+\end{array}$} & \multirow{2}{*}{$\begin{array}{c}\begin{array}{c}\text { Total } \\
\text { Strategy }\end{array} \\
+\end{array}$} & \multirow{2}{*}{$\begin{array}{c}\begin{array}{c}\text { Total } \\
\text { Capacity }\end{array} \\
+\end{array}$} & \multirow{2}{*}{$\begin{array}{c}\text { Promote } \\
+\end{array}$} & \multirow{2}{*}{$\begin{array}{c}\text { Undermine } \\
+\end{array}$} & \multirow{2}{*}{$\begin{array}{c}\text { CONMAX } \\
+\end{array}$} \\
\hline \multirow{6}{*}{3.2} & \multirow{6}{*}{$\begin{array}{l}\text { In control } \\
\text { and teacher } \\
\text { support, } \\
\text { from fall to } \\
\text { spring }\end{array}$} & Fall Construct & & & & & & & & \\
\hline & & Engagement & + & & & - & + & + & - & + \\
\hline & & Prob-Solving & & + & + & & & & & \\
\hline & & Info-Seeking & + & & & - & & + & - & \\
\hline & & Escape & & - & - & + & - & - & + & - \\
\hline & & Confusion & - & & & + & - & - & + & - \\
\hline & & & & rall Engageme & & Beh & vioral & & Emotior & \\
\hline \multirow{5}{*}{3.3} & \multirow{5}{*}{$\begin{array}{l}\text { In } \\
\text { Engagement } \\
\text { from fall to } \\
\text { spring } \\
\text { (feedback } \\
\text { effect)s }\end{array}$} & $\begin{array}{l}\text { Fall } \\
\text { Engagement }\end{array}$ & & & & & & & & \\
\hline & & Prob-Solving & & & & & & & & \\
\hline & & Info-Seeking & & & & & & & & \\
\hline & & Escape & & & & & & & & \\
\hline & & Confusion & & - & & & & & - & \\
\hline
\end{tabular}

Note: + = positive correlation $;-=$ negative correlation; blank space $=$ no significant association 
use of helpless ways of coping, and higher initial levels of Engagement leading to the use of mastery ways of coping.

Engagement as a mediator. A mediation model was tested to determine whether Engagement in the fall mediated the relationship between the two ways of coping that were noted as related to Achievement (Escape in the fall, InformationSeeking in the spring). Engagement was found to fully mediate the effects of both of these ways of coping on achievement.

\section{Goal 2: Coping and Perceived Control}

The link between the various aspects of perceived control and each way of coping were tested next. Correlations were examined first to determine direction and strength of relationships, followed by a series of regression equations to identify the predictive contributions of components of perceived control to ways of coping concurrently and over time. The pattern of relationships across all four ways of coping was similar, but variations in the combination of control components related to and predictive of each way of coping were noted.

Correlations. An examination of concurrent relationships between components of control and ways of coping showed that for Generalized Control beliefs, all Capacity beliefs, Effort Strategy beliefs, and the aggregate measures that represent profiles of control beliefs that Promote control and represent maximized control, positive relationships were noted with the mastery ways of coping, and negative relationships with the helpless ways of coping. One exception to this pattern was that 
Effort Strategy beliefs was positively related to Confusion. For all ways of coping, all Capacity beliefs and Generalized Control beliefs demonstrated notably stronger relationships than Effort Strategy beliefs, suggesting that all Capacity and Generalized Control beliefs have more influence in the development and promotion of mastery coping.

The Ability Strategy beliefs component was negatively related to InformationSeeking and positively related to both helpless ways of coping. A weak, positive relationship was also found with Problem-Solving. These relationships suggest that when a child believes that ability is necessary for success in school, he or she will be more likely to engage in maladaptive ways of coping than to seek out more information.

The relationships found for all the interaction terms for Strategy and Capacity beliefs follow the same pattern as the component relationships, with those terms indicative of attribution for success to internal causes related positively to mastery ways of coping and negatively to helpless ways of coping, and those terms indicative of attribution for success to external causes negatively related to mastery ways of coping and positively related to helpless ways of coping.

Escape coping showed the strongest correlations with all components of control except Effort and Ability Strategy beliefs, suggesting that a positive sense of control is particularly important to not giving up in the face of challenges or setbacks. In general, those children who have a perception of high internal control and low 
external control tend to use more mastery-oriented ways of coping, and those children whose perceptions tend to a profile of low internal control and high external control are, conversely, more likely to employ helpless ways of coping.

Predictive control components. A variety of techniques were employed to identify the predictive relationships between the components of control and the four ways of coping. Simultaneous regressions were conducted to identify the unique concurrent contributors to the variance in each way of coping; simultaneous backwards elimination regressions were used to identify for which known causes the interaction of strategy and capacity beliefs were uniquely related and predictive. Hierarchical regressions using all five of the aggregate measures of control were conducted to determine whether control predicts changes in each way of coping over time, from fall to spring, after controlling for the specific way of coping in the fall. For clarity, and due to the differential combinations of control components related to each way of coping, results are discussed here for each way of coping individually.

Problem-Solving. In the fall, predictive relationships were noted for both Total Strategy and Total Capacity beliefs to Problem-Solving, although Total Capacity beliefs was a considerably stronger predictor. These relationships, in spite of high correlations between the two aggregate control constructs, suggest that each makes a significant, unique contribution to the variance in this mastery way of coping. Within the strategy beliefs set, Powerful Others and Unknown Strategy Beliefs were negatively predictive of Problem-Solving, while Effort Strategy Beliefs was positively 
predictive. Within the capacity beliefs set, Effort and Ability Capacity Beliefs were both positively predictive. The aggregate measure that represents a control profile that Promote(s) control, and the interaction term for Effort also predicted positively to Problem-Solving in the fall, and the interaction term for Powerful Others predicted negatively. The same pattern of predictive relationships was noted for ProblemSolving in the spring. Problem-Solving in the fall was significantly related to Problem-Solving in the spring, yet all aggregate measures of control were predictive of changes in Problem-Solving from fall to spring, after accounting for the stability of this construct over time.

Information-Seeking. The pattern of predictive relationships was slightly different for Information-Seeking. In both the fall and spring, only Total Capacity beliefs was a positive predictor of Information-Seeking; Total Strategy beliefs did not show a predictive relationship. Within the strategy beliefs set, the same components that predicted Problem-Solving (Effort, Powerful Others, and Unknown Strategy Beliefs) were also predictive of Information-Seeking, in the same directions. In the spring, all the strategy beliefs were predictive, suggesting that as children get older, contributors to the variance in this way of coping become more differentiated and more influential. All capacity beliefs were positive predictors of Information-Seeking in the fall and spring, except for Luck Capacity Beliefs. The strength of prediction by Powerful Others Capacity Beliefs showed a marked decline. The same aggregate measure for the control profile that Promote(s) control that predicted Problem-Solving 
also predicted positively to Information-Seeking in the fall, along with the interaction terms for both Effort and Ability. The interaction term for Powerful Others also negatively predicted Information-Seeking in the fall. In the spring, these aggregate measures were joined by the aggregate measure representing the control profile that Undermine(s) a sense of control, which demonstrated a negative predictive relationship. Information-Seeking in the fall was significantly related to InformationSeeking in the spring, yet all aggregate measures of control were predictive of changes in Information-Seeking from fall to spring, after accounting for the contribution of Information-Seeking in the fall.

Escape. For this way of coping, Total Strategy beliefs was positively predictive, and Total Capacity beliefs negatively predictive, in both the fall and spring. Powerful Others, Luck, and Unknown Strategy Beliefs were all positive predictors at both time points, while all capacity beliefs in both fall and spring, were negative predictors, except for Powerful Others Capacity Beliefs in the spring. This reflects the strong correlations noted earlier, and supports the notion that external causes for success are more influential in promoting this way of coping. The aggregate measures representing control profiles that Promote and Undermine control showed strong negative and positive predictive relationships, respectively, with Escape coping in both the fall and spring. The strength of negative prediction by the Promote aggregate was noted to be increasing over time. The interaction terms for Effort and Ability predicted negatively, and the interaction terms for Powerful Others and Luck predicted 
positively, at both time points. Escape in the fall was significantly related to Escape in the spring, yet all aggregate measures of control were predictive of changes in Escape from fall to spring, after accounting for the contribution of Escape in the fall.

Confusion. The same pattern of prediction for Total Capacity and Total Strategy beliefs that was seen for Escape coping was noted for Confusion. All strategy beliefs except Powerful Others Strategy Beliefs were positively predictive of Confusion in the fall, with the same pattern noted in the spring, except that Luck Strategy Beliefs also dropped from the mix. The strong correlations of Effort, Ability, and Luck Capacity Beliefs with Confusion coping were reflected in the predictive relationships demonstrated at both the fall and spring time points, with the exception of Ability Capacity beliefs, which did not demonstrate a significant relationship with Confusion coping in the spring. The aggregate construct representing the control profile that Undermine(s) control, as well as the interaction terms for Effort and Luck were positive predictors of this way of coping at both time points, while the interaction term for Ability was a negative predictor in both fall and spring. Confusion in the fall was significantly related to Confusion in the spring, yet all aggregate measures of control were predictive of changes in Confusion over time after accounting for the contribution of Confusion in the fall.

\section{Goal 3: Coping and contextual factors}

To examine the linkages between contextual factors, i.e., forms of teacher support, and individual ways of coping, correlations were first examined for 
significant relationships, and then a series of multiple regressions conducted to determine which ways of coping could be predicted by concurrent measures of Teacher Support. These were followed by hierarchical regressions to test the predictive power of different forms of teacher support on changes in ways of coping over time.

Correlations. The construct for overall Teacher Support, as well as the components of Provision of Involvement and Provision of Autonomy were related to all ways of coping in both the fall and the spring, positively to the mastery ways of coping and negatively to the helpless ways of coping. Provision of Structure was related positively to Information-Seeking and negatively to Escape, but only in the fall. Consistent with correlations obtained throughout this study, the relationship of all variables with Escape was slightly stronger than other ways of coping at both time points.

Concurrent contributors. Multiple regressions tested the concurrent relationships between individual ways of coping and overall Teacher Support, Provision of Involvement, and Provision of Autonomy Support. Provision of Structure was not included in the models, due to the lack of significant correlations previously obtained. Only Provision of Autonomy Support predicted each way of coping when the other components of teacher support were included in the model. Teacher support predicting changes in coping. To test whether overall Teacher Support was predictive of changes in ways of coping from fall to spring, 
hierarchical regressions were conducted, with the fall measure of the particular way of coping being predicted included in the first step of the regression. For each way of coping, that way of coping in the fall was significantly related to coping in the spring. Teacher Support positively predicted changes in both mastery ways of coping, and negatively predicted changes in only Escape coping in the spring, over and above the variance accounted for by each way of coping in the fall.

Because Confusion coping was not predicted by provision of overall Teacher Support, the relationship was further examined by testing the individual components of teacher support for predictive power. Once again, no significant relationships for Provision of Involvement or Provision of Autonomy Support were found with Confusion, indicating this way of coping is not influenced by the provision of teacher support.

\section{Goal 4: Feedback effects of Engagement and coping}

The final set of analyses focused on identifying feedback relationships that would indicate that Engagement or the ways of coping shape the characteristics of their antecedents over time. Using the variables collected at the spring timepoint, overall Teacher Support, and the control aggregate representing a profile of maximum control, CONMAX, were regressed on Engagement and each individual way of coping to determine these reciprocal pathways in hierarchical regressions, accounting for the contribution of Teacher Support and CONMAX in the fall. 
Teacher Support. Engagement and Information-Seeking positively predicted changes in Teacher Support; Confusion negatively predicted changes, and both Problem-Solving and Escape were not related to changes in Teacher Support. These findings suggest that students who are more engaged with their school work and actively seek out additional information to complete their tasks are more likely to elicit additional support from their teachers.

To further explore the non-significant findings for Problem-Solving and Escape, the regressions were repeated using the individual components of Provision of Involvement and Provision of Autonomy Support in place of overall Teacher Support. Escape negatively predicted changes in both of these components of teacher support. Problem-Solving positively predicted changes in Provision of Involvement, but had no relationship with Provision of Autonomy Support.

Control. In spite of the high stability of the relationship between the fall and spring measures of the aggregate control constructs, Engagement still positively predicted, and the helpless ways of coping negatively predicted, changes in CONMAX. The mastery ways of coping were not related. To further explore these findings, the component aggregates for CONMAX representing the control profiles that Promote and Undermine control, and the aggregates for the total effects of the capacity and strategy components were substituted in the analyses for CONMAX. The same pattern of relationships as was found in the previous regressions for Engagement and the helpless ways of coping were noted. Information-Seeking 
showed a positive relationship with changes in the aggregate that represents the control profile that Promote(s) control, and a negative relationship with changes in the aggregate that represents the control profile that Undermine(s) control and the Total Strategy aggregate, but no relationship with changes in the Total Capacity aggregate. These findings suggest that control resources are in fact shaped over time by how a student copes with academic challenges, especially by a student's level of engagement, and when the student resorts to the helpless ways of coping.

\section{Summary of Study Results}

Students who are more engaged with their academic activities and who typically use more mastery ways of coping, particularly Information-Seeking, are more likely to receive increased teacher support over time, and build up their personal resources regarding perceived control. These students also enjoy increases in engagement over time, and more easily re-engage with academic tasks when faced with challenges or setbacks. Students who use Information-Seeking coping in particular may enjoy higher levels of achievement as well. Conversely, students who have a hard time staying engaged and who tend to employ Escape or Confusion as a means of coping with academic challenges and setbacks (e.g., giving up) are more likely to experience less support from their teachers over time, which contributes to the steady erosion of their internal resource of perceived control. Additionally, the use of Escape coping is particularly damaging, and is more likely to lead to increased disaffection over time, and lower levels of achievement. 


\section{Individual models for ways of coping}

The components of perceived control were found to be related to each of the four ways of coping according to different patterns. Differences in the relationships between coping and the other constructs of interest were also noted. These results led to the formulation of four separate models, one for each way of coping, to illustrate these different patterns of influence. These models are depicted in Figures 5-8. Double headed solid arrows indicate associations between variables (correlations), single headed arrows indicate predictive paths, and dotted arrows indicate feedback pathways. The bolded names of the control components indicate those that were most strongly related to the coping construct. The presentation of each model is accompanied by a summary of the most notable features of that model.

Problem-Solving. Four things are of particular interest in the Problem-Solving coping model (Figure 5). First, the findings that Problem-Solving coping did not show an association with Achievement, nor did it predict changes in Engagement over time were contrary to expectations. This researcher surmised the strong correlations found between Problem-Solving and Engagement arose from the strong feedback effect of Engagement on changes in Problem-Solving coping.

Second, strong relationships evidenced for Effort Strategy Beliefs and Effort and Ability Capacity Beliefs indicate the importance that effort and ability play in the initiation and maintenance of Problem-Solving coping. Moreover, the strong relationships found for the interaction score for Effort clarifies this association further, 
in that when a child believes effort is an effective strategy for success, and believes he or she is capable of exerting sufficient effort, the use of Problem-Solving coping is likely to increase. Additionally, both the aggregate measures for Total Strategy and Total Capacity positively predicted changes in Problem-Solving coping from fall to spring, further supporting the strong influence of control beliefs on this way of coping. Conversely, Powerful Others Strategy Beliefs was negatively associated with Problem-Solving coping, and the interaction term for Powerful Others negatively predicted changes in Problem-Solving coping from fall to spring, indicating that when children believe that their success is under the control of other people who have authority over them, and that they are not capable of influencing those people, Problem-Solving coping tends to decrease.

Third, Provision of Involvement and Provision of Autonomy Support appear to be particularly important in facilitating Problem-Solving coping in a student. The aggregate measure for overall Teacher Support showed a strong positive association with Problem-Solving coping, and when the aggregate was broken down into its component subscales and tested individually, the measures of Provision of Involvement and Provision of Autonomy Support showed the same positive association. Additionally, Provision of Autonomy Support positively predicted changes in Problem-Solving coping from fall to spring, suggesting that when a teacher is sensitive to a child's preferences regarding participation in and completion of 


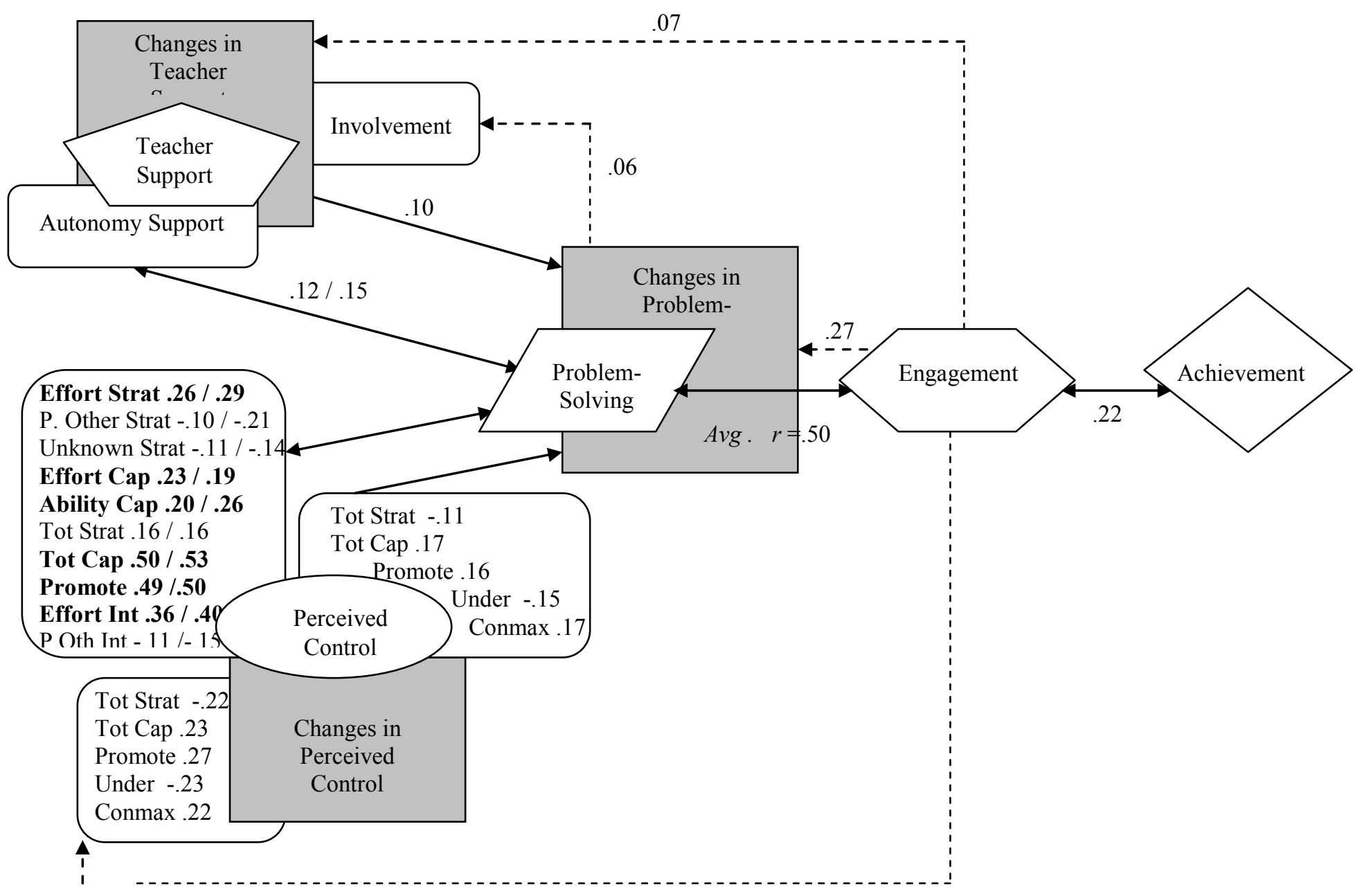

Figure 5: Final process model for Problem-solving coping (Greene, 2007). 
academic tasks, the use of Problem-Solving coping is likely to increase.

Finally, in addition to the feedback pathway noted from Engagement to Problem-Solving coping, a feedback pathway was confirmed from Problem-Solving coping to Provision of Involvement, indicating that children who frequently use Problem-Solving as a way of coping with academic challenges tend to receive increasing amounts of involvement from their teachers. Interestingly, a feedback pathway was not found from Problem-Solving coping to any of the control aggregates, suggesting that the use of Problem-Solving coping does not add to a child's sense of control.

Information-Seeking. The results for this model of coping were very similar to those obtained for Problem-Solving coping, and are depicted in Figure 6. While most of the same pathways were confirmed, three major differences emerged. First, unlike Problem-Solving coping, Information-Seeking coping in the spring was associated with Achievement. Therefore, a mediational model could be tested to determine the extent to which Engagement mediates the effects of Information-Seeking coping on Achievement. Results indicated that the effects of Information-Seeking coping on Achievement are fully mediated by Engagement.

Second, the specific control components associated with and predictive of Information-Seeking coping differed slightly from those for Problem-Solving coping. While Powerful Others Strategy Beliefs was associated with Problem-Solving coping, it was Unknown Strategy Beliefs that showed a strong, negative association with 
Information-Seeking coping. Powerful Others Capacity Beliefs also showed a similar relationship. This is a particularly bad combination of associations, as it indicates that a child who does not know what strategies may lead to success, also may not feel he or she has the capacity to influence powerful others in their life to help them discover those strategies. Additionally, the interaction score for Powerful Others was negatively predictive of Information-Seeking coping, suggesting that when a child believes powerful others in his or her life are in control of success, and he or she is not able to influence those powerful others to help him or her succeed, that child is less likely to engage in Information-Seeking coping activities whereby the help that is needed could be accessed. This researcher suggested these findings highlight the relational nature of Information-Seeking coping, as opposed to the activities of Problem-Solving coping, which represent a more solitary effort.

The third major difference between the models for the mastery-oriented ways of coping is in the feedback pathways evident for each. Problem-Solving coping showed no feedback effects on control, whereas Information-Seeking coping predicted changes in the control aggregates that Promote and Undermine control. This suggests that children who engage in Information-Seeking coping have a stronger sense of selfefficacy than those who don't, and that Information-Seeking coping is more influential than Problem-Solving coping in shaping a child's control resources over time.

Information-Seeking coping also predicted changes in overall Teacher Support, suggesting this way of coping exerts a more global influence than Problem-Solving 


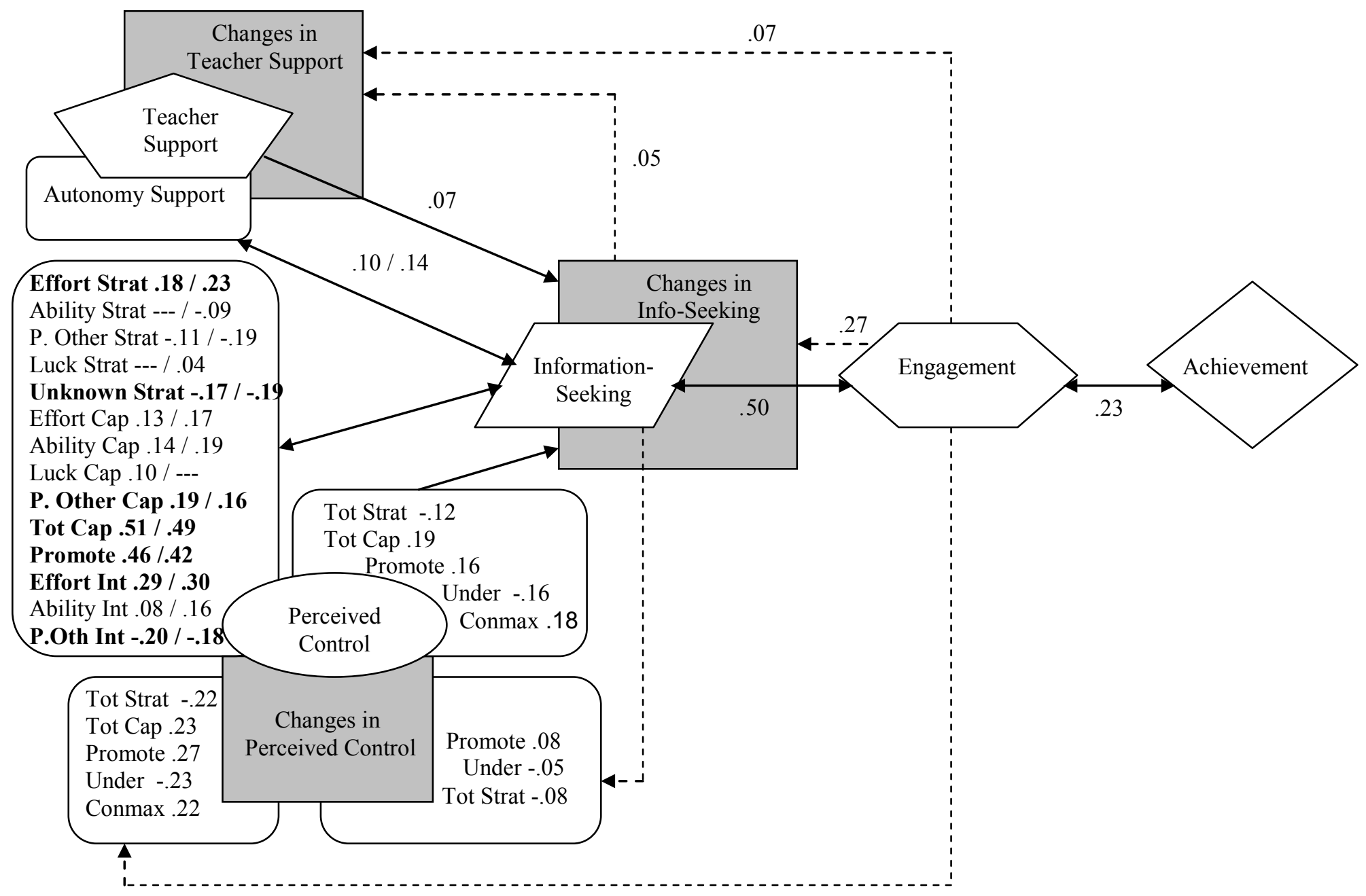

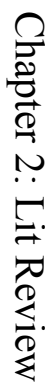

Figure 6: Final process model for Information-Seeking coping (Greene, 2007). 
coping in shaping teachers' responses to children's individual ways of coping. This is probably a reflection of the relational nature of Information-Seeking coping activities, as this researcher suggested earlier. Because of the close similarities in the models for each of these ways of coping, this researcher suggested that the mastery ways of coping are similar in their functions and effects.

Escape. The model for Escape coping, shown in Figure 7, evidences some of the strongest relationships and pathways of all the ways of coping, forewarning of the decidedly negative consequences of a consistent pattern of Escape coping. Escape coping was negatively related to both Engagement and Achievement, suggesting there may be a mediational role for Engagement in the relationship between Escape coping and Achievement. When a mediational model was tested, Engagement was found to fully mediate the effects of Escape coping on Achievement, just as was found for Information-Seeking coping. Escape coping also predicted changes in components of Engagement from fall to spring, specifically Behavioral Engagement. This relationship appears to be reciprocal, in that Engagement shows a feedback effect on Escape coping, suggesting that for behaviorally disaffected children, Escape may be a coping strategy of choice.

The most pronounced difference between the mastery-oriented models of coping and the Escape coping model is in the relationships with the components of control. In fact, all the components of control except Effort and Ability Strategy Beliefs were strongly related to Escape coping in the expected directions, suggesting 
that when a child attributes success to external, uncontrollable, or unknowable causes, increases in Escape coping are likely. The strongest predictor of Escape coping was the control profile representing the combination of control beliefs that Undermine control. The control aggregate for Total Capacity Beliefs and the aggregate representing a control profile that maximizes control were both strongly negatively related to changes in Escape coping, and the Undermine control aggregate was positively related to changes in Escape coping. This highlights how children who come to the classroom with a low sense of control or low confidence in their capabilities to master academic challenges are at risk for getting caught in a negative amplification cycle whereby these low levels of personal resources lead to increases in the use of Escape and withdrawal coping strategies, fostering increases in disaffection, which then feed back into their sense of low control and use of Escape coping strategies. Ultimately, learning and achievement suffer and it is very difficult for the child to break free from the effects of the negative amplification cycle.

Teacher Support was found to be an influential negative predictor of Escape coping, both concurrently and over time. Results for the teacher support constructs were similar to those of the mastery-oriented ways of coping, and in the expected direction.

Feedback effects for Escape coping were also strongest of all the models of coping. Escape coping predicted changes in all the control aggregates, and of particular interest, in both Provision of Involvement and Provision of Autonomy 


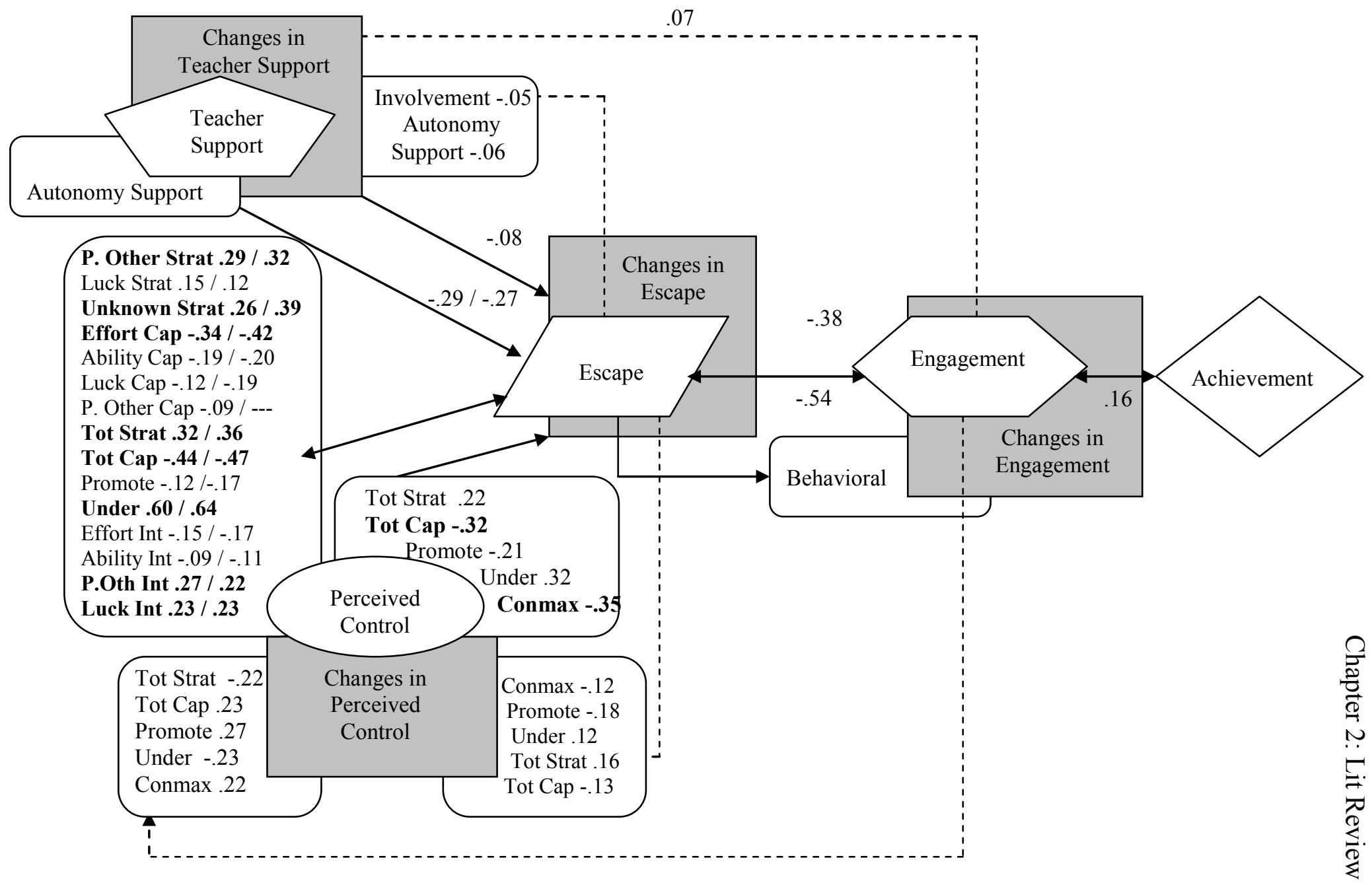

Figure 7: Final process model for Escape coping (Greene, 2007). 
Support; the mastery-oriented ways of coping only predicted changes in overall Teacher Support. This suggests that when teachers are less involved with their students and more coercive about classroom activities, children are more likely to evidence increases in Escape coping strategies.

Confusion. The confirmed pathways for Confusion coping closely mirror those of the Escape coping model and are illustrated in Figure 8. Three notable exceptions stand out. First, Confusion coping was not related to achievement as Escape coping was; therefore, a mediational model involving Engagement could not be tested.

Second, Effort Strategy Beliefs was positively related to Confusion coping, rather than in the expected negative direction, as was found for Escape coping. This researcher interpreted this surprising finding as suggesting a moderating role for effort in the relationship between Unknown Strategy Beliefs and the helpless ways of coping. It was argued that since Effort Strategy Beliefs were related positively to both Problem-Solving and Confusion coping, but not at all to Escape coping, it is possible that an inverse process involving Problem-Solving and Confusion coping is occurring. If Problem-Solving Efforts go unrewarded, the negative relationship of Unknown Strategy Beliefs with Problem-Solving may begin to increase in strength, triggering a parallel increase in the negative influence of Effort Capacity Beliefs, as the student may begin to question his or her capacity to exert sufficient effort. Left unchecked, this cycle is likely to eventually lead a child into Escape coping and withdrawal. 


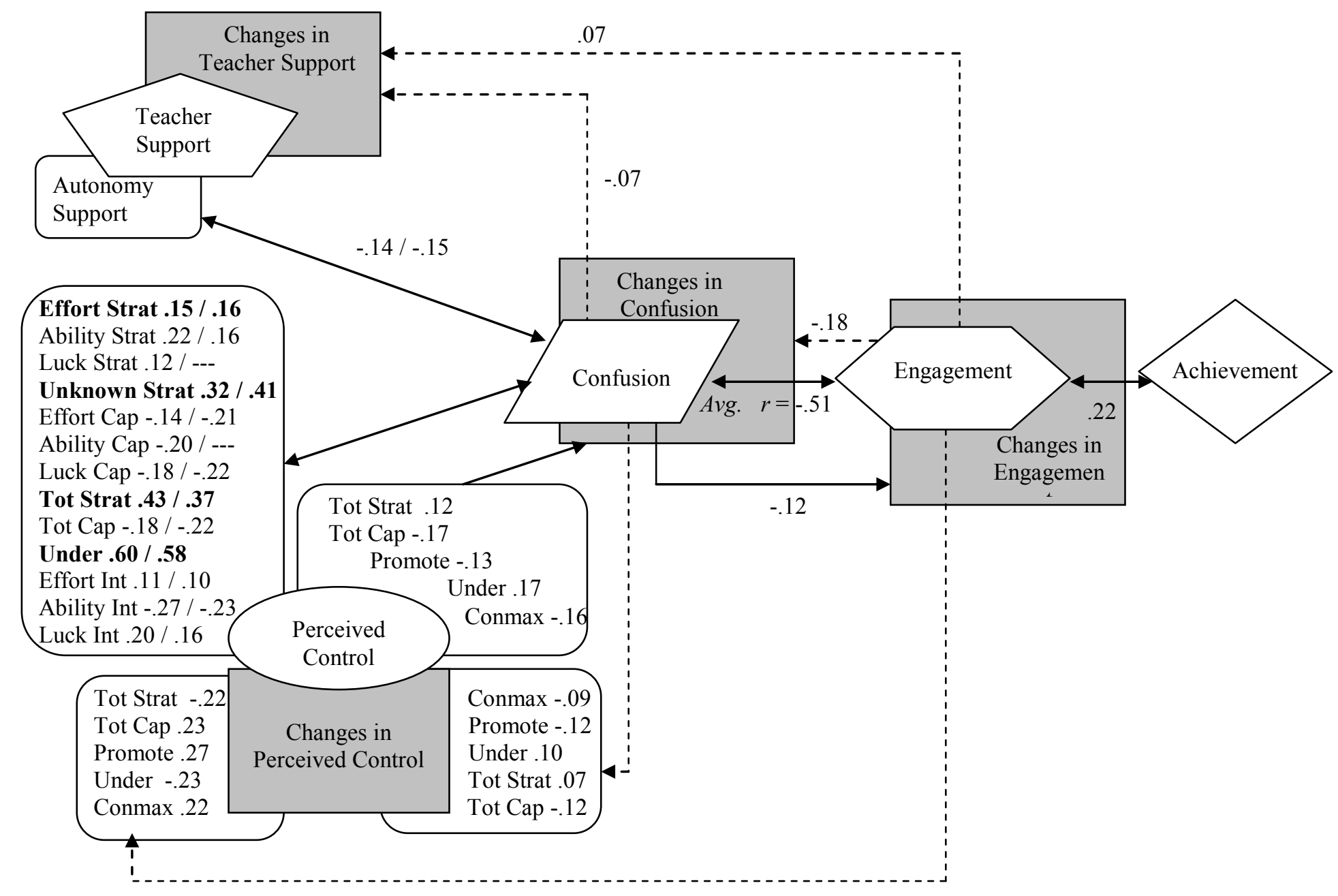

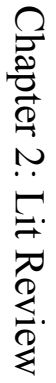

Figure 8: Final process model for Confusion coping (Greene, 2007). 
However, when Effort Strategy Beliefs are strong, this may have a moderating effect on the negative influence of Unknown Strategy Beliefs, and in turn, negative Effort Capacity Beliefs. The ultimate outcome then, when Effort Strategy Beliefs are strong, is that strong Unknown Strategy Beliefs will more likely lead a child from Problem-Solving coping to Confusion coping rather than to Escape behavior.

The third difference is of less impact, although worth noting. The feedback pathway from Confusion coping to Teacher Support showed prediction of changes in overall Teacher Support, whereas Escape coping predicted to Provision of Involvement and Provision of Autonomy only. All other relationships and predictive paths confirmed for Escape coping with Teacher Support, and the feedback pathways confirmed for Escape coping with control, were also confirmed for Confusion coping Conclusion of Literature Review: Part 1

In 2007 as part of an unpublished Master's thesis study, this researcher tested and confirmed a general process model applicable to the academic domain, illustrating the relationships between contextual support, components of perceived control, ways of coping, engagement, and academic achievement. A process study of this nature, situating coping within this context, has not been conducted previously. Key findings resulting from this inquiry have important implications for the present study.

One key finding that carries forward to the present study is that the process links of the model function differentially for each way of coping. Four models, one for each way of coping, were specified to illustrate these differential relationships. 
Different facets of control were found to facilitate mastery-oriented and helplessness ways of coping, and within that distinction, there were further differences in the components of control that distinguish between each of the mastery-oriented ways of coping and each of the helplessness ways of coping. Additionally, different patterns of control predicted changes in each of the ways of coping from fall to spring, as did contextual support. Unexpectedly, ways of coping were not strongly related to achievement.

Differential relationships between ways of coping and Engagement were also found. Mastery-oriented coping was found to facilitate Engagement, while helplessness coping showed deleterious effects on a child's motivation. While Problem-Solving and Information-Seeking showed similar relationships with Engagement, the relationships of Escape and Confusion coping showed differential effects, reflecting the different control profiles that contribute to each.

In addition to feedforward effects, feedback effects were also tested. All ways of coping except Problem-Solving showed feedback effects on control and contextual support, illustrating how the personal resources of coping and control shape each other over time and are influential in eliciting on-going support from teachers. Engagement also showed strong feedback effects on contextual support, coping, and control, but the reciprocal effects of coping on changes in Engagement included only the helplessness ways of coping. These findings provide strong evidence to suggest that coping and control are important to a child's motivational system and the elements 
that support it, and prompt the next logical question for investigation, which is how these relationships and processes change as a function of age. It is this question that is taken up by the present study.

Another key feature of this study that has importance for the current study is the conceptualization of coping according to the hierarchical model of coping families proposed by Skinner et al. (2003). Previous studies of coping effects or ways of coping have focused on maladaptive coping, rarely suggesting that there may be ways in which people cope that have benefits beyond the immediate dispatch of negative circumstances. By employing this framework, this researcher was able to detect differential antecedents and outcomes of adaptive (mastery-oriented) versus maladaptive (helplessness) ways of coping. These findings and the use of such a framework offer support for the existence of positive coping, or ways of managing one's reactions to stressors that allows for the growth and strengthening of internal resources. How this process unfolds over time is of interest to the present study, and the hierarchical framework of families of coping provides a viable platform from which to launch such an investigation.

\section{Literature review: Part 2}

The literature review in the next part of this chapter proceeds in two distinct sections, with each section corresponding to one of the stated goals of this study. In the first section, a review of the literature exploring developmental differences in perceived control, coping, and engagement is undertaken. These studies make an 
important contribution to the foundation of theory and research from which specific hypotheses in the present study have been formulated about the mechanisms by which the constructs of interest exert their influence on development. This section is modeled as a discussion of the normative development of the three target constructs, each in turn. Findings are summarized before moving to the second section of this part of the literature review.

Offered in the second section of this part of the chapter is a review of the few studies that have examined developmental differences in the constructs of interest in relation to changes in the hypothesized process links between them. While the total sum of this literature is small, it is sufficient to confirm the notion that age-related changes may not be limited to just the mean levels of the constructs. It is possible that the process structure itself may be subject to change. For example, the social context may have a stronger influence on coping when children are young, whereas selfsystem processes may play a bigger role as children enter middle school. Most process models assume that the processes are stable over any developmental change in the mean levels of the variables. The present study tests hypotheses that directly challenge this assumption, and which are suggested by the literature reviewed in the second section of this chapter. 
Normative age differences and changes in perceived control, coping, and academic engagement

The collective understanding in each of these fields of how and why development proceeds has evolved at widely disparate rates. Each field has grappled with issues that have either propelled the field towards a clear description and explanation of the developmental mechanisms at work (as in the case of perceived control), or stymied advancement to the point of bringing the field to a near halt (as has occurred in the field of coping). This section reviews selected articles in each field that describe the progress made towards an understanding of developmental trends, from both a descriptive and explanatory perspective, as well as the issues that have contributed to the lack of progress in some fields.

Normative Development of Perceived Control

Skinner, Zimmer-Gembeck, and Connell, 1998. The field of control is the most well-defined field with respect to development and age-related changes in various forms of control. This landmark study conducted by Skinner and her colleagues has provided the field with a definitive description of how the development of perceived control normatively proceeds. Using a latent growth curve modeling approach, growth curves were computed for the composite construct representing maximum perceived control. These analyses showed that perceived control increases steadily from third to fifth grade, peaks and begins to decline in fifth and sixth grade, and sharply declines between sixth and seventh grade. These results describe the 
normative progression of the development of overall perceived control for children in the academic domain in grades three through seven.

This same type of analysis was also conducted for each of the individual control beliefs of the tri-partite conceptualization, to better understand how different facets of perceived control develop over time. Results indicated that overall, control beliefs and capacity beliefs start out at higher levels than strategy beliefs, and remain as such. Both sets of beliefs showed relative stability until the end of $5^{\text {th }}$ grade, at which time they began to decline slightly. Within capacity beliefs, all beliefs except Luck exhibited this pattern, which began to decline more sharply between $4^{\text {th }}$ and $5^{\text {th }}$ grade. Capacity for Powerful Others began to decline about the same time, but not as sharply as capacity for Luck. Capacity for Effort showed the most decline over time, beginning between $5^{\text {th }}$ and $6^{\text {th }}$ grade.

Strategy beliefs were generally low to begin with, except for strategy for Effort, which began high and increased slightly over time. Ability was the next highest strategy belief and was relatively stable over time. Strategy beliefs for Powerful Others and Luck decreased until grade 5, when they began to increase slightly. The most interesting changes in strategy beliefs were for Unknown strategies, which decreased (improved) in $3^{\text {rd }}$ and $4^{\text {th }}$ grade, but began increasing (getting worse) at the beginning of $5^{\text {th }}$ and each subsequent grade. During fifth grade, a slight decrease was noted, but the pattern of increase returned at the beginning of $6^{\text {th }}$ 
grade and continued through $7^{\text {th }}$ grade, suggesting that doubt about how to be academically successful accompanies school transitions.

The development of perceived control can be expected to follow a predictable pattern as a child moves through childhood into adolescence. The findings described here outline the normative development of the components of perceived control in children in grades 3 through 7. Overall, perceived control declines over time, with a sharp decrease experienced at the transition to middle school. Young children tend to overestimate the amount of objective contingency between their actions and subsequent outcomes, and this tendency is reflected in the high levels of capacity beliefs that are present until the end of middle childhood. At this time, capacity beliefs become more realistic and children understand they do not have as much control over uncontrollable causes (Luck and Powerful Others) as they once thought. This decline in belief about the controllability of uncontrollable factors is accompanied by slight increases in the belief in the power these factors may have over success, as strategy beliefs for uncontrollable causes tend to increase over time. This trend, in conjunction with high strategy beliefs for Ability may contribute to the erosion of a child's belief in their ability to exercise effective effort, which declines sharply at the middle school transition.

Normative Development of Coping

Developmental changes in ways of coping are not well understood despite twenty years of studying coping in children and adolescents. Disagreement about how 
to conceptualize and measure coping has stalled progress towards a comprehensive description of the development of coping (Skinner \& Zimmer-Gembeck, 2009). The majority of the empirical work has focused on individual differences in specific, predetermined ways of coping and their concurrent connections to adaptive and maladaptive outcomes; however, consensus around which ways of coping are most developmentally appropriate for which age groups has not been reached, contributing to the current problem of multiple approaches to studying coping which cannot yet benefit from each other. For example, on one hand, there are many studies that investigate the ways of coping for particular age ranges, yielding descriptive information about what types of coping strategies are most prevalent at what ages. On the other hand, many studies are conducted comparing across age ranges, but when different ways of coping are used from study to study, this makes comparisons between studies is nearly impossible (Fields \& Prinz, 1997).

In the absence of a framework that defines a common core of ways of coping that are developmentally age-graded, integration of these multiple approaches to describe normative developmental trajectories of coping processes is not possible. Skinner and Zimmer-Gembeck (2007) sum up this dilemma in a recent review of the work in the field: "These studies, because they utilize a wide variety of partially overlapping coping categories and a wide variety of largely unselected age groups and gaps, have proven difficult to integrate” (Skinner \& Zimmer-Gembeck, 2007, p. 16). 
Attempts have been made to interpret the work to date, and to speculate about what normative development in coping might look like, in spite of the disparity in samples and methodology. Four reviews have summarized the available literature and offer comment on apparent developmental trends (Fields \& Prinz, 1997; Compas, et al., 2001; Skinner \& Zimmer-Gembeck, 2007; Zimmer-Gembeck \& Skinner, 2011).

Fields and Prinz, 1997. In a review of over 40 studies, Fields and Prinz (1997) attempted to outline developmental trends noted in the literature. The studies they reviewed were classified into three types: descriptive and taxonomic studies, age group studies, and those investigating the coping-adjustment relationship. Descriptive and taxonomic studies rely on checklists of possible ways of coping that children endorse in relation to specific stressors. These studies are more descriptive than developmental, as they are concerned only with what strategies are used by what age groups and do not engage the types of analyses that would provide information on how strategy use changes over time. Age group studies compare and contrast strategy use in different age groups. These studies yield descriptive information about what strategies are most prevalent at what ages, but are still more descriptive than explanatory, as they cannot specify the mechanisms through which one strategy declines while another emerges as the child moves from one age group to the next. Adjustment studies are not concerned so much with development as they are with describing how differences in strategy use result in differential outcomes in adjustment and well-being; an individual differences approach. 
After evaluating the selected studies, the authors offer a synthesis of the findings that describes apparent trends in development from preschool through young adolescence. Overall, a progression is noted that begins with concrete, problemfocused and / or avoidance strategies in the preschool years, moving to more emotionfocused, approach, and cognitively based strategies as a child matures. Instrumental action declines in favor of strategies aimed at emotion-regulation, while reliance on social support steadily declines over time, shifting from adults to peers and back to adults again. Differentiation of coping skills increases over time, with an accompanying ability to match appropriate coping skills to specific stressors.

Of particular interest to the present study are the changes that occur in the shift between late school age (9-10) and adolescence (12-13). During this shift, more complex cognitive strategies come online, and children continue to differentiate their coping repertoire, acquiring new strategies as they age. Behavioral avoidance strategies have declined, possibly supplanted by cognitive avoidance tactics; approach strategies, such as direct problem-solving, continue to be frequently employed. Children at this juncture rely on social support less and less, but their preferred social resource shifts once more from peers back to adults. As children cross into adolescence, their use of and capacity for cognitive strategies continues to increase, while the overall variety of coping strategies employed begins to decrease. Adolescents also become more proficient at matching their coping efforts to particular 
stressors, most likely due to advances in cognitive development and the emergence of formal operations.

Ultimately, Fields and Prinz (1997) concluded that while some developmental trends seem to be evident, no definitive conclusions can be made, due to a variety of conceptual and methodological issues. They advocate the use of caution when integrating study findings, citing the competing goals of defining the normative development of coping processes, and linking coping strategies to adjustment outcomes as just one of many issues that prevents the coping field from making progress on any front.

Compas, Connor-Smith, Saltzman, Thomsen, \& Wadsworth, 2001. Compas, et.al. (2001) reviewed a variety of issues in the field of coping and evaluated over 60 studies to assess progress with respect to the relationship between coping and adjustment. These authors were particularly interested in the extent to which the findings from these studies could be compared with respect to adjustment outcomes, although the importance of a developmental synthesis was recognized. A summary of developmental trends noted in those few studies identified as developmental was outlined, largely congruent with the more detailed synthesis offered by Fields and Prinz (1997). However, these authors also make clear the difficulties inherent in a true developmental synthesis, stating:

Differences in coping as a function of age (or developmental level) are also important to consider. Similarities and differences in coping as a function of age 
should help to define the developmental course of coping...However, in spite of the fundamental importance of understanding age effects and individual differences in coping, research in these areas has been disappointing, primarily as a result of problems in the conceptualization and measurement of coping. Because of the limitations of research in these areas, we have not addressed them in this review. (Compas, et al., 2001, p. 81)

The limitations mentioned are discussed at length in the article and encompass the issue of categorization schemes that not only overlap in conceptualization and definition, but which were established based on the work on adult coping, offering no provision to accommodate developmental changes. Also discussed are the disparities found from study to study in measurement instruments, assessment methods, and statistical issues, rendering the body of empirical findings non-comparable. Furthermore, the authors point out that the majority of studies done in the field of coping are cross-sectional in design, which precludes the determination of direction of influence, and therefore, of development.

Skinner and Zimmer-Gembeck, 2007. Expanding on the notion that the coping field is slowly converging on a general definition of coping as "regulation under stress," Skinner and Zimmer-Gembeck (2007) first present a framework that integrates dual process models of regulation with the hierarchical family model of ways of coping (Skinner et al., 2003). This integration highlights coping as a coordinating concept for specific subsystems of regulatory processes that target behavior, emotion, 
attention, and cognition, in that when these subsystems work together synergistically, they produce the actions described by coping. Furthermore, by invoking the hierarchical family model of coping, a framework is provided that is flexible enough to accommodate differences in the manifestations of the combination of regulatory subsystems that reflect age-graded, developmentally appropriate and functionally similar responses to stress.

Using this integrated, multi-level framework, these authors evaluated 44 studies of coping that reported age differences. To compare studies and identify normative trends in the development of coping responses, these authors had to resort to an item-by-item analysis of the subscales used to measure coping in each of the studies to identify ways of coping that are comparable according to the hierarchical family model of coping. Ways of coping were coded according to the families, and particular times of developmental transitions were noted.

From a general survey of the developmental literature on regulation, 5 developmentally critical age periods where shifts in regulatory strategies are most prominent emerged: infancy and toddlerhood (to age 2), middle childhood (age 5 to 7), late childhood to early adolescence (age 10 to 12), early to middle adolescence (age 12 to 16 ) and middle to late adolescence (age 16 to 22). Different regulatory subsystems are implicated at different age periods and for different manifestations of action, accompanied by different facilitating contributions from a variety of social partners. 
For the age period of interest to the present study (mid-to-late childhood), these authors identified a developmental pattern that includes an increase in cognitive coping, making it possible for children to intentionally coordinate their coping efforts with the actions of others. They also noted that as children move into adolescence, meta-cognitive capacities emerge, allowing children to include future concerns, others' reactions, and long-term impacts in their selection and implementation of coping strategies.

Support-seeking. A more detailed examination of study findings for this age period involving specific ways of coping revealed that patterns in coping responses are largely context specific. In particular, support-seeking was found to follow the same pattern as described by Fields and Prinz (1997), where children in middle childhood tend to seek out peers more than adults, but as they make the transition to adolescence, adults become increasingly important once again. This trend seems to be most prevalent when children are dealing with uncontrollable stressors, and as children move farther along the continuum of adolescent development, they become better able to identify those situations where adult support would be most helpful.

Problem-solving. With respect to problem-solving, when problem-solving was measured as cognitive activity rather than direct instrumental action aimed at changing the situation, age related increases were found, particularly between middle childhood and early adolescence, increasing steadily throughout the adolescent period. When problem-solving was measured as instrumental action, or in conjunction with 
behavioral problem-solving and other factors (e.g., ambition, commitment), agerelated declines were detected, suggesting that the more cognitively advanced forms of coping are most strongly associated with older age groups.

Escape. When children were asked to recall their own experience of a stressful situation, declines in the endorsement of escape coping were found in relation to increasing age, particularly for those children over the age of 12 . One exception to this was found for adolescent children coping with cancer, where increases in escape coping were detected. When children were asked to report on coping with particular domains of stress rather than their own specific situations, no age differences were found.

Distraction. Comparable to the age-related increases found in cognitively based problem-solving, cognitive distraction was found to follow a similar pattern of increase as children got older, particularly for those children moving from middle childhood to adolescence, and continuing through early adolescence. Interestingly, behavioral distraction was also found to increase with age rather than decline, as might be expected as cognitive capacity emerges. This highlights the increasing capacity of children to selectively employ coping strategies based on the nature of the stressor as they get older. During the transition to adolescence, more use of other strategies such as cognitive restructuring, blaming others for problems, and self-reliance was also noted, although studies utilizing scales for these ways of coping were sparse, and caution in interpreting these results is recommended. 
These findings suggest that further examination of how relationships between variables change during these critical periods may lead to a process model that holds promise for understanding the development of coping capacity. However, until issues previously discussed are resolved, a comprehensive description of developmental trends in coping will continue to be elusive. Nonetheless, the development of coping for children moving into late childhood and across the transition to adolescence can be expected to follow a general pattern characterized by an increase in cognitively based strategies with a corresponding decrease in behavioral means of coping. Additionally, as children mature, the ability to select coping responses appropriate to the stressor becomes more refined, and the repertoire of coping strategies available remains welldifferentiated.

Zimmer-Gembeck and Skinner, 2011. In an update to their 2007 review, these authors continue the discussion of challenges in the field, outline the benefits of a truly developmental body of research on coping, and apply their multi-level framework of coping to the task of integrating findings from 58 studies seeking to comment on the development of coping. Their efforts confirmed the most evident transition points for the age groups previously identified: 1) infancy and toddlerhood (about age 2); 2) ages 5 to $7 ; 3$ ) late childhood to early adolescence (about ages 10 to 12 ); 4) early to middle adolescence (about ages 14 to 16); and 5) middle to late adolescence ( about ages 18 to 22). The present study is most interested in the results of integrating findings for the third age group, late childhood to early adolescence. 
Using the hierarchical families framework of coping to organize the categories of coping reported in each of the studies, all categories were coded into the lowerorder ways of coping most closely suggested by the families framework. Then, findings within each family of coping were ordered as a function of age in order to note developmental windows where processes suggested by theories of regulation might be more clearly recognized. Next, they separated "pure" scales (measurement scales that tapped coping from only one family) from scales that mixed measurement of ways of coping from multiple families, or also measured other constructs within the same scale. Method of assessment was taken into account, as were other factors related to coping at different ages.

From an analysis of correlation patterns among ways of coping in studies reporting age differences, conclusions were drawn about the qualitative age-related shifts in coping that might represent developmental trends for each of the developmental periods identified. Results confirmed the observation from the previous review that the most frequently used ways of coping come mainly from four families: problem-solving, support-seeking, distraction, and escape. However, they also noted more frequent use of coping from additional families: accommodation, selfreliance, submission, and opposition.

Patterns for each of the families of coping were reported from two perspectives: first, for those studies where "pure" measures were used, and second, for studies that used mixed measures of coping. Patterns noted in these authors' previous 
review were confirmed, with additional information gleaned about the situationspecific nature of some ways of coping, in addition to the difference in results when "pure" measures are used compared to combinations of ways of coping from different families.

Problem-Solving. When pure measures of problem-solving activity were used, problem-solving was seen to increase for self-identified stressors or when multiple stressors were encountered. This increase was seen beginning with middle childhood and extending into young adulthood. When the stressor was of an uncontrollable nature (e.g., parental cancer), no age differences or decreases were noted. When measures of problem-solving also included measures of support-seeking, a difference between problem-solving with instrumental versus emotion-focused support-seeking emerged. For children in early to middle childhood, the use of problem-solving with instrumental support-seeking was seen to increase, while problem-solving with emotion-focused support-seeking led to decreases or no age differences for this same age group. Problem-solving activity in combination with other cognitive forms of coping led to increases with age, while the inclusion of emotion-focused coping strategies or escape coping led to no age differences, or decreases during adolescence.

Distraction. Very young children were found to make frequent use of behavioral distraction as a means of coping with stressful situations, which increased with age as children's ability to move to different locations of their own volition increased. When behavioral distraction was distinguished from cognitive distraction 
in studies using pure measures, behavioral distraction was found to be the most commonly used way of coping for children beginning at early ages, and enduring throughout childhood and adolescence, thus no age differences were found in most studies, although a few did report increases across these age periods. In studies focusing only on cognitive distraction, increases were reported for children across early and middle childhood (ages 5 to 11) for situations involving uncontrollable medical circumstances, and when behavioral distraction was not an option. When behavioral distraction was an option, cognitive distraction showed no increases with age. For studies that measured both behavioral and cognitive distraction together, the type of stressor seemed to be key to which form of distraction was used, and whether that behavior increased or not across age groups. When the stressor was seen as uncontrollable or a serious matter, distraction was seen to increase if it was not already the most common way of coping reported. When the stressor was more general, interpersonal, or self-identified, no age differences were found. In addition, behavioral distraction was more often actually used than cognitive distraction, by all age groups.

For studies that combined measures of distraction with accommodation or strategies for emotion-management, increases during adolescence were found primarily when distraction was measured as cognitive distraction; when distraction was measured as behavioral, results were stable. When combined with avoidance coping strategies, increases were found during younger age periods (ages 4 to 10) 
when the goal of coping was to avoid problems, while decreases were seen in older children (age 7 and older) when avoidance was aimed at escape.

Support-seeking. Support-seeking was found to be the most commonly used strategy for dealing with distress by young children, as would be expected from theories of attachment. Moving into the range of childhood, in studies using pure measures of support-seeking, decreases were found for the early to middle childhood age group (ages 5 to 12), particularly for stressors involving achievement, peers, selfidentified issues, and uncontrollable circumstances. No age differences were found when compared to adolescence. When measures of support-seeking were combined with measures of adaptive problem-solving, increases were found during age periods where problem-solving is expected to increase, but decreased if the stressor was noted as an interpersonal problem, pain, or uncontrollable. When combined with emotionfocused coping strategies, decreases were seen all across middle childhood to middle adolescence.

A consideration of the source of support revealed very few studies that distinguish between support-seeking from peers versus support-seeking from adults. However, in the few studies that did specify support-seeking from peers, it was observed that the preference for the source of support followed patterns established by other researchers (Fields \& Prinz, 1997; Skinner \& Zimmer-Gembeck, 2007) in that young children most often seek support from adult caretakers and attachment figures, which shifts to peers as children enter later childhood and early adolescence, and then 
shifts back again to adults as children move into middle and later adolescence. Older adolescents also exhibit a tendency to seek out advice and support from trusted adults and professionals in fields pertinent to their growing ability to plan for the future.

Escape. Escape coping was found to be the most common maladaptive way of coping used all across the age ranges; however, in comparison to other ways of coping, in general, it was not very commonly used in childhood or adolescence. Most studies using pure measures of escape measured cognitive forms of escape, and overall, decreases or no age differences were found for all age ranges. One exception to this pattern where increases were found was for the late childhood and early adolescence age ranges, although reported use of escape was still low. When combined with other forms of maladaptive coping, decreases across age groups became more evident, and began at an early age (about age 4). Again, the only findings of increases in escape and maladaptive coping were for early adolescents, particularly in the academic domain. When escape was measured along with distraction, it was more likely that increases would be found, although the instance of decreases, primarily during adolescence, was still more prevalent.

Accommodation, self-reliance, submission, and opposition. In this updated review, additional families of coping were noted in the studies examined, and patterns of developmental progression for these families of coping were outlined. Accommodation includes strategies such as cognitive restructuring, positive self-talk, and focusing on the positive aspects of a situation. Self-reliance includes self- 
regulation of emotions, keeping feelings to oneself, and accepting responsibility for problems and solutions. Submission encompasses rumination, worry, and giving up, and opposition encompasses aggression and blaming others. Of these, children and adolescents most often reported using strategies of accommodation, but measurement of this and the other families of coping were most often combined with measures of other ways of coping.

Age related increases were found for accommodation strategies across a wide range of ages (age 4 through adulthood) when combined with measures of problemsolving, distraction, social support-seeking, and emotion regulation. When measured as a pure strategy or as a minimizing strategy rather than a positive self-talk strategy, no age differences were found from early childhood through late adolescence (ages 4 to 20). Self-reliance was rarely measured as a pure measure, and was usually combined with emotion expression or regulation strategies. Older children were found to make more frequent use of this way of coping, with increases found for children in middle adolescence. Submission as a way of coping was diverse in its measurement; when measured as rumination, no clear pattern was discernible; when measured as worry, showed increases from late childhood through adulthood; when measured as giving up, was found to be rare, but stable when detected, in late childhood and early adolescence, increasing slightly as children transitioned into adolescence. Opposition measured as aggression in response to stress was found to decline from early to middle childhood and remained relatively low until early adolescence, when verbal forms of 
aggression were noted on the rise in response to peer conflict and school problems. No age differences were found across middle to late adolescence in opposition in response to family stressors, peer problems, and self-identified stressors.

Coping profiles. These authors make the argument that there is a difference between studying profiles of coping and conducting research on individual differences in specific ways of coping. They argue that the study of coping profiles is what will develop the body of research that is needed to advance the developmental study of coping. To illustrate their point, they integrate the findings of their review of studies according to families of coping into specific, age-graded profiles of coping. Of particular interest to the present study are the profiles of coping for children in middle childhood and early adolescence.

In sum, children in middle childhood exhibit an increasing differentiation in cognitive forms of coping as development of cognitive capacities continues to unfold. Cognitive forms of problem-solving and attention-regulation develop and are added to instrumental strategies, and maladaptive forms of coping are seen to decrease. With an expanding ability to match environmental contingencies with available resources, they become increasingly more selective in their support-seeking activities. Preference for social partners is seen to increase for adults at early stages, then shift from adults to peers, although adults are still preferred when the target of social support is for emotion-focused purposes, which is seen to decline. As children get 
older, they are increasingly more independent and self-reliant, and their coping repertoire becomes increasingly more sophisticated.

As children enter adolescence, instrumental forms of coping increasingly give way to more cognitive based forms of coping, such as planning and cognitive problem-solving. As their coping repertoire continues to diversify, they rely on both cognitive and behavioral distraction tactics when needed. Support-seeking activity shifts back towards adults and professionals they interact with as they become increasingly able to consider potential life pathways. Regulation processes become more complex and sophisticated, with accommodation and self-reliance becoming more prevalent as means of intentionally managing emotions and cognition. Coping becomes more flexible as adolescents become more proficient in recognizing specific features of stressful situations and matching their coping efforts to effectively manage them. In contrast to increasing flexibility, however, is evidence of more entrenched patterns of typical coping responses, as earlier forms of coping do not completely disappear. While earlier forms of coping may decrease in favor of more sophisticated strategies based in emerging capacities, it is possible that those earlier forms of coping may become established, default responses, particularly in situations of extreme stress, regardless of whether they are ultimately the most adaptive response. There is, however, evidence to suggest that when faced with extreme stress, the coping responses learned early in life are in fact the most adaptive response in the moment. One additional observation pertinent to the present study is that in early adolescence, 
there is a noted increase in less adaptive ways of coping, as children in this transition phase are trying to manage stressors arising from internal processes as well as from external sources, and the rate at which early adolescents are able to negotiate this transition and the vulnerabilities it brings varies widely.

\section{Normative Development of Engagement}

Unlike the coping field, engagement researchers seem to be in general agreement about the conceptualization of engagement as a construct. Most researchers accept engagement as a multidimensional construct, comprising three different types: behavioral, emotional, and cognitive. However, issues around specific definitions of these three types still exist, and convergence on appropriate measures for each type has not occurred, making it difficult to identify the specific mechanisms that facilitate engagement and allow researchers to chart its development across a child's school career.

Fredericks, Blumenfeld and Paris, 2004. These issues are discussed in greater detail in a review of the state of the field by these authors, who illustrate how, from study to study, the construct has not been conceptualized in a consistent manner. Behavioral, emotional, and cognitive engagement have been defined broadly, and no one measure encompasses all three types, or all facets of each type. Additionally, while some studies have measured two of these types of engagement together, most investigations look at only one type of engagement, in isolation from the others. This precludes an examination of the synergistic (additive or compensatory) effects of all 
facets of engagement operating simultaneously that would be possible in a processoriented analysis.

Wigfield and colleagues, 2006; 2015. In spite of these challenges, researchers continue to work towards defining normative processes of development in children's academic engagement. The research that has been done in the larger field of achievement motivation has been instrumental in defining engagement and laying the foundation for an expanding research agenda. Wigfield and his colleagues periodically review the research concerning the development of achievement motivation; in their review of 2006, they organize their discussion around three primary themes: theoretical perspectives, the development of motivation, and the socialization of motivation (Wigfield, et al., 2006). In the section addressing development, Eccles work with person-environment fit models is cited, describing how engagement typically suffers a sharp decline across the transition to middle school (Eccles \& Midgley, 1989; Eccles, et al., 1993).

In the most recent installment of this review, the discussion moves to the exploration of a series of questions that explore the most influential developmental process that underlie achievement motivation and student engagement (Wigfield et al., 2015). These authors reiterate the developmental pattern of engagement outlined in their 2006 review: engagement typically suffers a sharp decline as children get older and move towards the transition to middle school. They also initiate a discussion of how associated declines in students' motivational beliefs and values, including their 
beliefs about the causes of success, and their own capacity to employ those causes to their benefit, are related to declines in engagement. Their conclusions correspond with the predictable patterns of development in control beliefs and associated outcomes on engagement as found by Skinner et al. (1998).

The normative developmental trajectory of academic engagement for children in grades 3 through 7 has been established by Skinner, et al. (1998) in their study regarding the development of perceived control. Using the same latent growth curve approach described previously, growth curve estimates were calculated using mean level differences in children's engagement as reported by both students and teachers. Growth curves were calculated individually for each set of reporters, and were largely consistent, showing that engagement starts out in $3^{\text {rd }}$ grade at a relatively high level and remains steady through $5^{\text {th }}$ grade, when it begins to decline over the transition into middle school. This finding is consistent with the previous research of Eccles and her colleagues (Eccles \& Midgley, 1989; Eccles, et.al., 1993), and the hypotheses formulated for the present study reflect similar expectations.

\section{Summary of normative development of target constructs}

Definitive description of the normative progression of development has been accomplished with respect to perceived control. Such progress in the field of coping has been slow to emerge, due to the lack of consensus on categories of coping and appropriate methods for measuring coping at different ages. Promising frameworks and theoretical integrations have been advanced over the past decade, however, and 
general descriptions of developmental trends in functionally similar groupings of coping strategies have been outlined. There are still many issues to be resolved, and a comprehensive body of research that establishes the normative developmental patterns for coping is not yet a reality, although attempts at integrating the work to date have demonstrated the utility of promising methodologies and clearly set the direction for future work.

In the field of engagement, the problems are less perplexing, and there is substantial evidence that points to the normative declining pattern of engagement as described by the reviews of Wigfield and his colleagues $(2006 ; 2015)$. Outstanding issues in the operationalization and concurrent measurement of all facets of engagement, including how to integrate aspects of engagement such cognitive, psychological, and academic with behavioral and emotional engagement, must be addressed in future work to solidify an understanding and definitive description of the developmental progression of engagement as a holistic construct. It is encouraging that the field is becoming more aware of and interested in extending the scope of research in engagement through the consideration of topics such as re-engagement, the neurophysiological basis of the underlying processes of motivation, and reciprocal influences of the myriad factors that shape engagement and motivation (Skinner \& Pitzer, 2012; Wigfield et al., 2015). Advances in understanding these related topics will move the field of engagement to a whole new level in the future, opening exciting 
opportunities for practitioners in the field of education to be more effective in their efforts to inspire students in the endeavor of learning.

Changes in process links between perceived control, coping, and academic

engagement

Process analyses in control. In the landmark study of Skinner and her colleagues (1998), the general process model depicted in Figure 1 was used to specify a model from which the normative progression of development for perceived control was explored and documented. In their study, the specification of that model was teacher as context, perceived control as self, engagement as action, and academic achievement as outcome. An investigation of the predictors of change in the links of the process links relating these constructs, and how they operate differently for different age groups, yielded a description of changes in the process structure. The following paragraphs describe the findings for the processes analyses examining the different links between control and the other components in the model.

Control and engagement. Regression analyses testing age-graded models of 2year trajectories of engagement were compared to detect age interactions in the process link between components of control and engagement. Results indicated that the process does in fact differ between younger children (3rd and 4th grade), and older children (6th and 7th grade). The control profiles that predict the slope of the trajectory of engagement did not differ between the groups (high capacity beliefs predict better trajectories, high maladaptive profiles predict worse trajectories, and 
changes in positive control profiles predict changes in the trajectory). However, the types of control beliefs important to each age group were different. Strategy beliefs were found to be more important to establishing the direction of the trajectory for younger children than for older, and changes in capacity beliefs were found to be more important for older children than for younger children. These results suggest that the process by which control influences engagement operates differently at different ages. A more fine-grained analysis that investigated the specific control beliefs in each case was conducted to pinpoint what type of strategy or capacity belief was responsible for the age differences found. Through this analysis, these authors were able to describe the specific control profiles that operate at different ages, and demonstrate how, as children age, fewer variables are able to exert an influence on the shape of their engagement trajectories, highlighting the importance of paying close attention to the development of children's self-system processes at an early age.

Teacher context and control. These same analyses were conducted for the link between teacher context and perceived control, with teacher context as the predictor. Results indicated that with respect to control beliefs and capacity beliefs, the prediction was the same for both age groups, although the strength of the relationship in all cases was stronger for younger children than for older. With respect to strategy beliefs, however, an interesting difference between the two groups emerged. For uncontrollable strategies (Powerful Others, Ability, and Unknown causes), the relationships were significant at the $p>.001$ level for the younger children, compared 
to either non-significant relationships or significance at the $p>.01$ level for the older children. These results demonstrate how the context is more important at an early age for the development of adaptive uncontrollable strategy beliefs.

Achievement and control. Parallel analyses were conducted using academic achievement as the independent variable and perceived control as the dependent variable to test for feedback effects of performance on control. Results indicated that for younger children, prior performance was most strongly related (negatively) to the development of Unknown strategy beliefs, suggesting that their beliefs that they do not know what it takes to be successful are increasingly confirmed by their previous poor performance. For older children, prior performance unexpectedly was most strongly associated (positively) with capacity for Powerful Others, suggesting that as children get older, they increasingly associate good performance with getting teachers to like them. Effects were stronger for older children than for younger children, indicating that individual performance is increasingly more important to children's perceived control as they get older.

Process analyses in coping. This kind of analysis in the field of coping is rarely attempted. The review articles cited previously that describe the issues in the field highlight the reasons why the coping field has not been ready to approach the integrated study of process and development with respect to coping capacity. A search of the literature returns very few studies that incorporate coping and control in the academic domain, let alone from a developmental, process-oriented perspective. 
Skinner and Zimmer-Gembeck (2007) and Zimmer-Gembeck and Skinner (2011) provide insight to the field on what still must be done in order to approach such a task, and illustrate the utility of the hierarchical family framework of coping in achieving that outcome (Skinner et al., 2003). In another article of 2011, these authors take the next step towards that end, and tackle the question of the process link between perceived control and coping.

Skinner and Zimmer-Gembeck, 2011. In a brief introduction, these authors present evidence supporting the notion that control, whether objective or subjective, and the experience of exercising control, is a crucial determinant of how people face stressful situations, and the degree of success they have in effectively meeting the demands stressful encounters present. Having established this link between stress and the experience of control, they then suggest that normative developmental shifts in perceived control might be useful as markers of related normative shifts in the development of coping behavior. The proceeding discussion revolves around three key themes: how mastery oriented and helplessness coping change in form across developmental age periods, how the development of perceived control contributes to shifts in coping as people age, and how coping may exert reciprocal influences on and shape the development of perceived control.

Shifts in mastery and helplessness coping. When coping is considered as an outcome of a particular profile of control, these authors suggest that changes in that control profile can help identify changes in the form of coping across age transitions, 
as the link between control and the function that coping serves is believed to endure. This notion is best illustrated by a look at the development of control and coping in infancy. Infants are hard-wired to detect action-outcome contingencies in the environment and are genetically and temperamentally predisposed to react to stressors by initiating a test of those contingency relationships. Their earliest actions involve exploring whether the environment (caregivers) is responsive to their needs by providing warmth, nourishment, and caring, or is hostile, neglectful, and indifferent. These early experiences with the environment set the stage for the development of control expectancies that will amplify over time with repeated experience. When their signals of distress are met with needed comfort, they come to expect that meeting their needs is under the contingent control of their own initiation of distress signals. Caregivers serve as co-regulators in the process of coping, and a mastery-orientation in the infant is strengthened.

Conversely, when their signal of distress is repeatedly met with neglect or hostility, this sets up an expectation of non-contingency, which leads to a helplessness orientation over time. Moreover, repeated experience of non-contingency and a persistent sense of helplessness diminishes infants' ability to detect future contingencies, thereby reinforcing a negative pattern of coping and control behaviors.

During middle childhood, which is the most relevant age period to the present study, the experience of control is influential in shaping the selection of problemsolving strategies, which are beginning to shift to more cognitive forms of problem- 
solving as cognitive capacities continue to develop. If a child's control profile has been shaped by iterative experiences of non-contingency, then it may be difficult for the child to correctly identify an effective problem-solving solution, and precludes the possibility that they can produce evidence of their own effectiveness that would disconfirm their beliefs about the extent of control they are able to exercise. As these negative experiences with coping and control replicate, a helplessness orientation continues to gain a foothold and can be particularly difficult for social partners to intervene in, given that coping strategies are shifting to more cognitive based forms that may be more difficult to detect. In the classroom, for example, students are not usually free to just get up and physically leave the room, and therefore must resort to mental withdrawal of effort or daydreaming if they are prone to escape behavior. Teachers may not be fully aware that this process is occurring and may not respond in ways that successfully bring students back to a point of reengagement with their academic tasks.

Perceived control and coping. In the next section of this chapter, the authors review the composition of perceived control in relation to the functions its components serve in an action sequence, such as a coping episode, and relate the features of the development of control to the process of coping at different ages. Beliefs that come prior to the initiation of a coping action are considered regulatory beliefs. Beliefs that come after a coping action that make sense of what happened during the coping episode are considered interpretive beliefs. Generalized control beliefs regulate action 
and help a person launch a coping effort. Strategy and capacity beliefs are interpretive beliefs and help explain why a particular outcome was achieved, or a desired outcome was not achieved, and the role the person had in producing the end result.

These sets of beliefs can be combined at levels that are optimal, and facilitate mastery experiences, or can be at levels that are not adaptive and lead to helplessness. An optimal control profile is one where generalized control is high, beliefs about the causes of success focus on effort, and there is strong confidence in one's capacities. This profile has been found to be strongly related to achievement outcomes in the academic domain in previous research by these authors (Skinner et al., 1998). A control profile where generalized control is low, strategies for success focus on external, uncontrollable causes such as luck or powerful others, and where confidence in one's capacities is low is a profile commonly associated with a helplessness orientation.

The developmental course of perceived control is well established. Young children generally have a high sense of personal control and high confidence in their own abilities, which often is not supported by the objective indicator of actual performance, and an undifferentiated view of what causes success. The intensity of a young child's own wishes and desires fuels their belief in their own effectiveness and bolsters their general sense of control. Over time, changes in these conditions occur as children come to understand the various factors that can have a causal effect on outcomes. They come to realize that it takes more than just personal force in some 
cases to achieve a desired outcome, and no amount of wishing it true will make it happen. Through this process, the strength of the belief in their own effectiveness begins to wane, as they become better able to evaluate their actual performance and adjust their expectations about their capacities. As the process of differentiating causes of success continue, and as effort is recognized as independent of effort, capacity beliefs decline, uncertainty about the causes and controllability of success arise, and the notion that anything can be accomplished through personal force is adjusted to be more realistic.

Embedded in these change processes are three well-documented shifts in the development of control that may serve as markers for shifts in the development of coping. First, the differentiation of causes of success includes distinguishing between the self and others as the causal agent that produces outcomes. This process typically occurs during the second year of life, and may be responsible for the characteristic need of the toddler to attempt tasks on his own, without the help of an adult. This shift marks a move to more self-reliant, independent coping by the child, although at young ages, before a history of competent independent action has been built up, children need patient caregivers standing by to provide instrumental help if called upon, in order for the child to have a successful experience of independence. When done with care and sensitivity to the child's needs, and intentional feedback attributing success to effort and strategy, and not to ability or other fixed traits, a successful history of these types of coping episodes can lead not only to increased confidence in one's capacities, 
but also to a healthy sense of being able to access powerful others when needed, and the role that they have in assisting with coping. Caregiving actions that are intrusive, coercive, and are not tolerant of the child's need for independent action risk leading a child to passivity, anger, and resistance. Caregiving that is neglectful leaves the child in a state of being overwhelmed and can lead to confusion, discouragement, and anxiety. These negative responses engender a helplessness orientation that is likely to amplify over time.

The second major shift in perceived control that may signal concomitant shifts in coping is the development of the ability for social comparison. This shift reflects the adjustment in a child's control profile towards a more realistic view of their own capacities and emerges in middle childhood. Evaluating personal performance against the performance of others and noting a level of performance that seems to be the norm, as well as judging where one's own performance falls in reference to that norm helps a child in the differentiation process of causes, particularly effort and ability. An understanding of the relationship of task characteristics to performance also provides valuable information about one's capacities in reference to others, as objectively difficult tasks should results in poorer performance for everyone, while performance that is falling behind may signal a need for more effort. When this ability becomes refined, coping efforts are more effective, as children can better gauge the requirements of a situation and the resources needed to effectively master it. The flip side of this advancement, however, is that for children who regularly lag behind their 
peers in performance can easily become discouraged, devalue their own competence, and fall into helplessness behavior. Social partners and contexts, particularly the classroom context, can help a child avoid the pitfalls associated with social comparison by focusing on the task characteristics and levels of effort required for mastery, and not on capacities needed, and by adopting learning orientations that help a child focus on improvement in their performance compared to their own past performance, rather than performance orientations that result in winners and losers. The typical practices in education of assigning grade rankings and grouping children by ability can inadvertently send the wrong message to students who tend to struggle in comparison to their peers.

The third development in perceived control that may serve as a signal of shifts in coping behavior is the further differentiation of the concept of ability. As the cognitive ability to infer meaning from disparate information comes online at the transition from middle childhood to early adolescence, an understanding of the inverse relationship between effort and ability emerges. Ability, as an unseen capacity is observed only via an inferential process of tracking a pattern of successful performance on normatively difficult tasks with low effort expenditure. Children come to realize that smart children do not need to expend a high level of effort to achieve the same outcome as other children who try harder. This sets up a dangerous causal chain of thinking for a child though, in that the next logical conclusion they come to is that if extra effort is expended to achieve a result, and in the end they fail, 
then they must not be very smart. This is a seriously damaging conclusion for a child's capacity beliefs, particularly if they believe that ability is a necessary cause of success. A pattern of repeated failures can cause children to adopt a helplessness orientation when faced with certain challenges, and can even extend to a more global state if left unaided. Younger children, who have not yet attained the capacity for inferential thought or the differentiation of effort and ability, associate success with being "good" and failure with being "bad." Hence, the effect of repeated failure for younger children is extremely damaging to their budding sense of self.

The next effort for these authors was to illustrate the reciprocal nature of coping and control. The cumulative history of control experience and the effect that has had on shaping control beliefs includes coping episodes as experiences of control. Those scenarios where a stressor was successfully dealt with strengthen a positive control profile, whereas those scenarios where coping efforts were less effective or maladaptive contribute to declines in perceptions of control. A particularly difficult coping scenario to process positively is one where the stressor is objectively uncontrollable. Work in the areas of secondary control and coping appraisals has shown that when people turn their focus to secondary goals that do offer a measure of controllability, and interpret the experience of non-contingency as an opportunity to learn something useful for future efforts, a feedback loop from coping to control is created that strengthens control beliefs rather than damages them. Social partners who 
help children focus their thought process on learning from mistakes and failures can benefit children's strivings towards an adaptive coping orientation.

Process analyses in engagement. Studies conducting process analyses between engagement and constructs of the motivational model used in the current study, aside from perceived control, are difficult to locate, as this type of investigation has not really taken hold in the research community as of yet. A book chapter written by Skinner and Pitzer, 2012, is presented here, as it was written as a guide to the motivational model and provides a discussion of what research that has been done in the field of engagement can contribute to future studies that seek to adopt a process oriented approach to examining the complex interplay of influences between model constructs involving engagement.

Skinner and Pitzer, 2012. In a chapter devoted to the explanation of student engagement as a motivational construct situated within a multi-level model of positive youth development, these authors walk the reader through the same motivational model as utilized in the present study, calling upon evidence from research that supports the influence of various forces that shape the development of engagement. Of particular relevance to the present study are the discussions of teachers as context, coping as everyday resilience, and the reciprocal influence of engagement on these important components in the motivational model.

Teacher context and engagement. These authors cite a variety of previous work that supports the proposition that teachers can facilitate motivational gains for 
students through supportive interactions that provide for their self-system needs. They suggest that pedagogical caring is supportive of the need for relatedness, optimal structure is supportive of the need for competence, and autonomy support scaffolds self-determined action, and cite research that has demonstrated all three forms of support to be important in shaping motivation and subsequent achievement. The quality of interactions then that teachers have with their students has an important influence on a student's level of engagement. When teachers offer learning activities that have inherent interest, are fun, and yet appropriately challenging, students are encouraged to follow their own interests and goals, and benefit when teachers also provide clear feedback or instruction on how to attain those goals. In addition, classroom contexts that promote the relevance and value of learning, even when academic tasks are not intrinsically motivating, foster gains in autonomy and selfregulated learning, which deepens the well of motivation a child can draw from in subsequent encounters with challenging academic work.

Coping and engagement. Coping in the academic domain is referred to by these authors as resilience, sometimes studied as everyday resilience, or academic buoyancy, and is characterized as a set of resources a student can access to help them bounce back after a setback or failure and to reengage with academic work. Taken together in its various forms, coping as a repertoire of behaviors can either be adaptive, allowing for effective management of stressors and continued engagement with challenging circumstances, or maladaptive, leading to a state of helplessness and 
disaffection from academic challenges. The nature of a child's coping profile, that is, the most common pattern of response to stressful academic circumstances, also exerts an influence on a student's level of engagement.

Reciprocal influences. Motivational dynamics are discussed by these authors as the feedforward and feedback loops among features of the self, context, actions and outcomes in the model that create amplification cycles that build engagement.

Supportive interactions with teachers promote gains in self-system perceptions, which are manifested in a child's control profile and which contribute to effective coping and result in high-quality engagement. High-quality engagement reinforces self-system perceptions and control beliefs of competence for the student, as well as reinforces teacher efforts at providing appropriate contextual support, and so the cycle continues. The amplification effect can work in reverse as well; when contextual supports are coercive or neglectful, or when control profiles focus on person-characteristics and uncontrollable causes of success, doubts about one's capacities increase and can deplete self-system resources, igniting maladaptive responses to stress and challenge, which in turn causes a student to withdraw from academic tasks. These effects will feed back onto the other components of the motivational system in negative ways to cause an amplification of disaffection that may become cyclical. Several studies are cited that capture snapshots of different parts of this process to support the notion of reciprocal influences. 
One area of particular importance to note is that of the feedback effects of a student's level of engagement on teacher motivation. Teachers are subject to the same self-system needs and processes outlined by the motivational model as students are, and student engagement provides information to them about their level of competence as a teacher. As such, teachers' own control and coping profiles may be shaped by student engagement, resulting in less than optimal responses when students are disaffected and unresponsive to teacher attempts to help them reengage with challenging tasks. This only serves to further undermine student engagement, thus setting up another amplification cycle of negative effects.

Summary of Process Links Research. The adoption of a process perspective requires particular attention to the dynamical nature of the constituent constructs and variables, and the interactions among them. Consideration not only of how variables and their relationships with each other change over time, but also of how changes in mean-levels of the variables (normative developmental trends) are related to changes in the way they interact (process-links) is critical to an investigation of process. Few actual studies in the literature approach the study of control, coping, or engagement from this perspective. Those few studies that do employ this perspective were reviewed, as well as articles discussing the underlying mechanisms of process at work. The general process model previously presented has been explored in sufficient detail with respect to control to allow for an understanding of how and when different facets of control become important for the facilitation of engagement, and 
how contextual factors promote or inhibit those relationships. The processes involved in the development of coping have not been investigated to the same degree; however, considerable work has been documented that integrates the research conducted in areas related to coping, and outlines an agenda for a productive line of inquiry to build the body of developmental research necessary to move the study of coping forward. Of particular interest and utility is the integrative work of Skinner and her colleagues $(2007 ; 2011 ; 2012)$, that attempts to outline how research efforts might approach studying the development of coping, by using the shifts in the development of perceived control as markers that signal shifts in the form of coping across developmental periods. The field has also been introduced to a promising framework for the conceptualization and measurement of coping based on process and developmentally oriented analyses that may ultimately lead to a clear picture of how coping capacity develops. Engagement as a robust construct that facilitates academic achievement continues to be explored as a motivational resource that energizes a child's relationship with the learning process and provides the necessary feedback information to amplify positive processes that produce desired outcomes. The process models used in this study, combined with the kinds of analyses illustrated here, hold great potential to further an understanding of children's motivational development in the academic domain. 
Conclusions from Literature Review: Part 2

Two main conclusions can be drawn from this review. First, more research from an integrated, developmental perspective is needed to support definitive conclusions regarding the direction of influence between the constructs of control, coping, and motivation. Longitudinal studies exploring reciprocal relationships have been sparse, and the findings from those studies that have hypothesized relationships of this type that include coping have not been consistent. This question merits further inquiry.

Second, a process approach is necessary to understand the complex dynamics of development and motivation. Understanding the disparate information imparted by analyses of individual differences, mean-level differences, and age interactions is essential to explicating process models that can accommodate the dynamic processes that direct the emergence, growth, and decline of capacities that are appropriate to the contextual demands presented at different ages, and which feed into the motivational system.

Progress towards this type of understanding in each field has occurred at different rates, with different levels of success. The field of control has been well defined with respect to these processes, while the fields of coping and engagement are still struggling to differing degrees to resolve issues of conceptualization and measurement. Definition of an integrative framework that can accommodate developmental shifts in coping behavior has been challenging and elusive, although 
recent research and documentation of carefully thought out integrations of bodies of research has provided promising possibilities. The present study seeks to employ a process-oriented, integrative model and framework that brings together previous research and theory in the control and coping fields, and which holds potential for contributing to the work that will advance the agenda for a developmental study of coping beyond current limitations. 


\section{CHAPTER 3: PURPOSE OF STUDY}

Considerable research has been conducted to investigate the relationships between children's perceptions of their abilities, what it takes to succeed in school, and how those beliefs influence motivation and academic achievement. Most researchers agree, based on the results of this research, that self-system perceptions (relatedness, competence, and autonomy) are critical to the formation and execution of motivated behavior in the academic domain. Substantial investigation of the impact of social partners on the development of achievement related beliefs and behaviors has also been conducted, yielding support for the notion that teachers' interactions with students are an important factor in the development of self-system perceptions, and subsequently, motivational outcomes of engagement and learning. Additionally, research on the role of coping behaviors in motivational processes in the academic domain has recently been conducted, with preliminary evidence suggesting that coping is a personal resource and an important piece of the motivational puzzle, warranting further investigation.

However, while these findings are important to the understanding of academic motivation and achievement processes, no systematic integration of these constructs has been undertaken to discern the combined and reciprocal effects of these factors over time that also incorporates an examination of how normative developmental changes influence the process relationships among them to effect differential outcomes. Using the same integrative, process-oriented approach employed in this 
researcher's unpublished Master's thesis study (Greene, 2007), which yielded the four models of coping depicting the relationships among the constructs of interest, the present study seeks to extend this approach to include an examination of how the process relationships of the component constructs in those models change when normative developmental changes in those constructs are accounted for.

Contributions of the present study. The present study is formulated to address existing gaps in the literature on the development of motivational processes, and to contribute in several important ways to the collective effort of the research community to understand the factors that influence children's academic outcomes. First, this study will contribute to the literature on children's coping by demonstrating the utility of a developmentally-graded system for the conceptualization and measurement of coping behavior. The hierarchical family model of coping as formulated by Skinner and her colleagues (Skinner et al., 2003), is a promising framework for conceptualizing coping from a developmental perspective. The present study relies on this framework for its conceptualization and measurement of children's coping, illustrating how this framework can allow researchers to detect and chart the developmental progression of the ways in which children cope with challenges and threats in the academic domain.

Second, the present study continues to build support for an action-theoretic motivational model that promotes the definition of coping as action-regulation under stress. The conceptualization of coping as a personal resource that connects a child 
with the motivational processes necessary to maintain or reestablish engagement with academic activities in the face of challenges and setbacks has been gaining attention, and research to date has yielded promising results that warrant further exploration. The previous work of Skinner \& Wellborn (1994; 1997), as well as this researcher's unpublished Master's thesis, adopts a developmental conceptualization of coping as a personal resource that can be shaped by interactions with features of the context (teacher support), which children may draw upon to negotiate challenging circumstances. This conceptualization of coping is situated within the framework of the Self-Systems Model of Motivational Development depicted in Figure 2, as proposed by Connell (1990), and Connell and Wellborn (1991). By incorporating a developmental focus in the research design, the present study seeks to provide continuing support to move this conceptualization towards wider acceptance by the research community as a productive framework for the investigation of children's coping processes, particularly those of a developmental nature.

Third, this study contributes to the understanding of how interactions with teachers and the type of support they offer their students can influence the developmental path of internal resources (coping and control), and how those interactions feed back over time to shape subsequent teacher behavior. An understanding of how this process impacts a students' engagement and learning in the classroom has been elevated to the level of national concern through federal policy and specific funding efforts to enhance teacher effectiveness (e.g, No Child Left 
Behind, Race to the Top grant program). As noted earlier, substantial research has been devoted to investigating the student-teacher relationship; however, understanding how that relationship changes with the development of the student has not been pursued with the same level of urgency. The present study seeks to examine whether development does in fact play an important role in the exchange over time between teachers and their students, and to explore how that exchange may foster or hinder positive outcomes for students. In addition, and of particular importance, is establishing an understanding of how the reciprocal influence of student behaviors and outcomes on teachers and their subsequent interactions with their students changes with child development, a line of questioning the present study includes in its design.

Lastly, the present study seeks to make a contribution to methodological considerations for future investigations by demonstrating how a process-oriented, developmental approach to exploring the relationships among the constructs of interest can yield information that cannot be obtained by other popular research designs. The model tested is a dynamic model, formulated from a systems perspective that takes into account feedforward, feedback, and reciprocal cycles. The study design is longitudinal, allowing for detection of within-subject developmental changes as well as age-graded inter-individual differences. When applied to an investigation of changes in the process structure that relates the constructs of interest in this study, a more robust understanding of children's motivational processes can be obtained. 
Previous studies, even those of a longitudinal nature, have not incorporated all these features in a systematic and fruitful manner.

Several research questions have been formulated that the present study is designed to address. The next section presents these questions, along with the expected outcomes of the planned analyses. The research questions are informed by and address the relationships depicted by the process model diagram presented in Figure 9. Expected results for specific hypotheses for each research question are summarized graphically in Tables 2-5, which are presented individually after each question. All hypotheses are summarized in words and presented together in Table 6 at the end of this chapter.

\section{$\underline{\text { CONTEXT }} \underline{\text { SELF }} \underline{\text { ACTION }}$ OUTCOME}

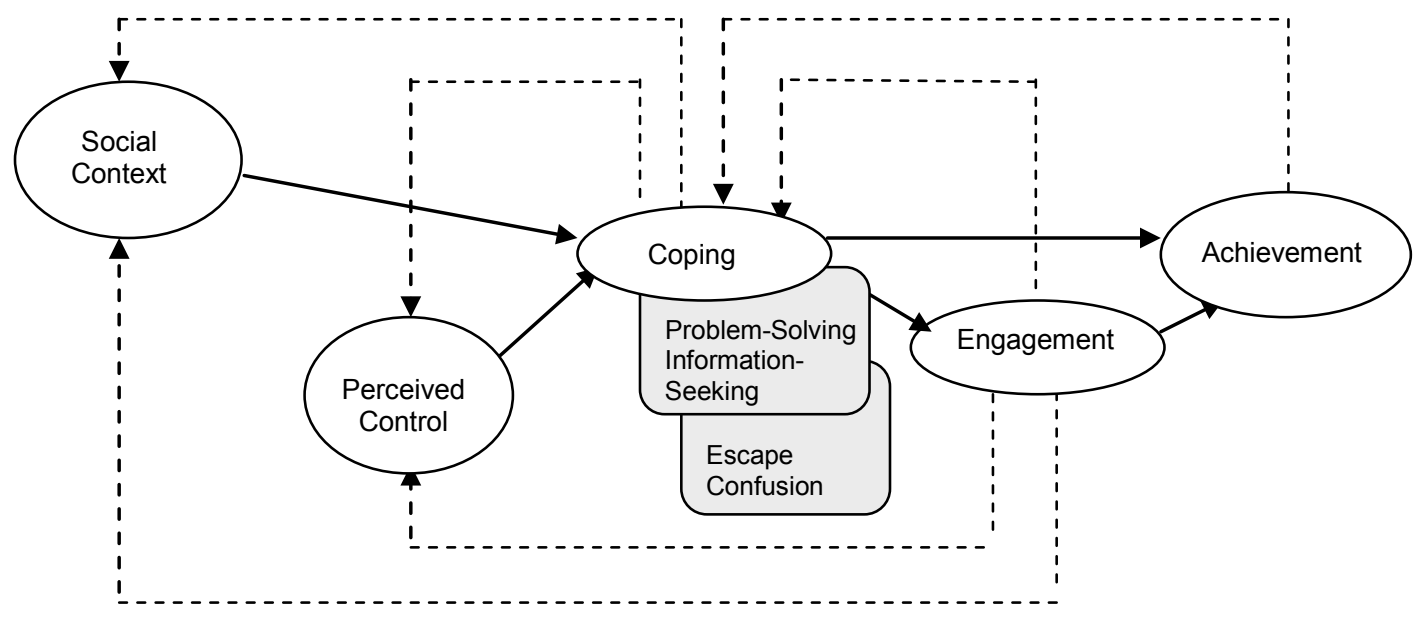

Figure 9: General process model that informs the formulation of research questions for the present study. Solid lines represent feedforward effects, dotted lines represent feedback effects. 


\section{Research Questions}

1. Are there age-graded differences in the relationships between ways of coping (mastery-oriented and helplessness), engagement, and academic achievement?

This set of hypotheses investigates how the mastery/ helplessness dimensions of coping influence academic engagement and achievement, and how changes in academic engagement over time differ according to age (measured by a proxy variable indicating the students' grade level at the time of measurement). Feedback effects of engagement and achievement on subsequent coping are tested, as well as a model of engagement as a mediator of the effects of coping on achievement. In general, children who employ mastery forms of coping such as Problem-Solving and Information-Seeking tend to sustain higher levels of engagement over time, and should ultimately experience a greater degree of overall achievement. Some support for the hypothesis that engagement acts as a mediator of the effects of some forms of coping on achievement was noted by this researcher's unpublished Master's thesis work, with higher levels of engagement found to promote subsequent increases in the use of mastery coping (Greene, 2007). However, it is unclear whether the strength of these relationships remains constant over time, or exhibit variations that correspond with normative developmental changes.

\section{1a) Coping and Engagement.}

This set of hypotheses tests both the feedforward effects of coping on engagement, and on changes in engagement, and the feedback effects of 
engagement on ways of coping. These relationships are illustrated using a causal loop diagram, shown in Figure 10. Causal loop diagrams are used to depict the dynamical nature of the relationships among variables. Figure 10 shows how increases in mastery coping lead to increases in overall engagement, which further strengthens mastery coping; conversely, increases in helplessness coping lead to decreases in overall engagement, which serves to strengthen helplessness coping. Both loops are reinforcing loops, as indicated by the small circle in the middle marked with " $\mathrm{R}$ " for reinforcing.

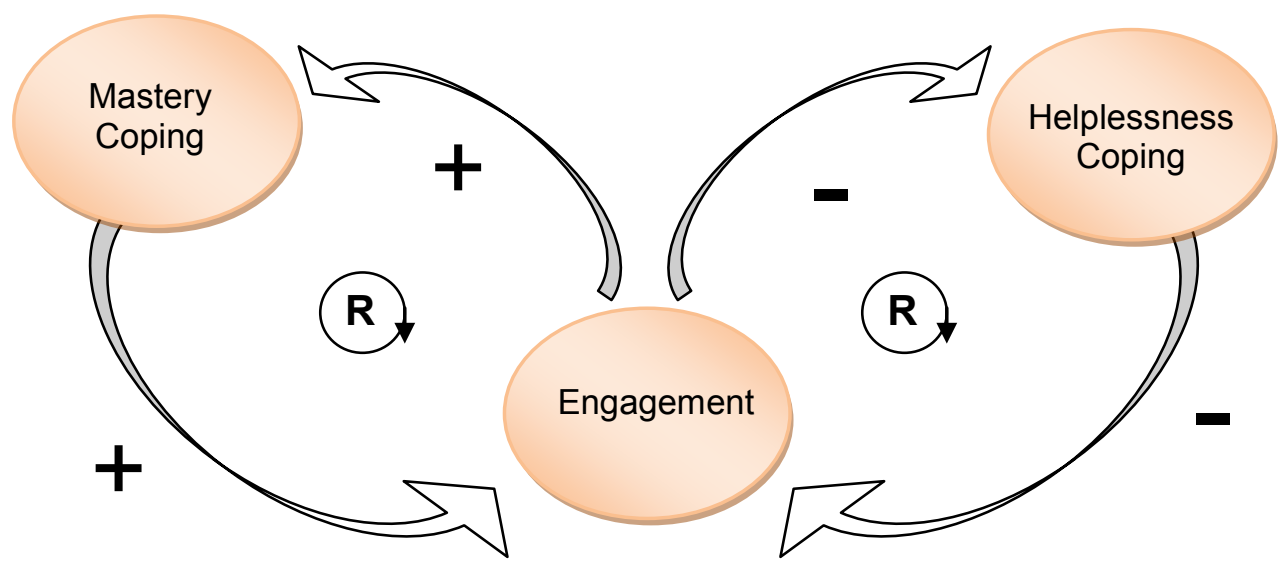

Figure 10: Causal loop diagram depicting dynamic feedforward and feedback relationships between ways of coping and engagement.

Coping predicting Engagement. In this researcher's unpublished Master's thesis work, a general pattern was noted between ways of coping and Engagement wherein mastery coping was positively related to Engagement, and helplessness coping was negatively related. In the current study, the relationship between coping and Engagement for all age groups is expected to 
follow this same general pattern. In addition, the relationship for both ways of mastery coping and for confusion coping with Engagement is expected to be stronger for older children than for younger children, based on trends identified in the literature that more cognitive forms of coping emerge as children age (Fields \& Prinz, 1997; Skinner \& Zimmer-Gembeck, 2007; Zimmer-Gembeck \& Skinner, 2011). Also based on these trends, the relationship between Escape coping and Engagement is expected to be stronger for younger children, as younger children tend to use more avoidance forms of coping than older children.

Coping predicting changes in Engagement. With respect to coping in the fall predicting changes in Engagement in the spring, no prediction is expected for the mastery ways of coping for any age group. For the helplessness ways of coping, it is expected that Escape coping will predict changes in Engagement for younger children only, and Confusion coping will predict changes in Engagement for older children only, over and above the effects of levels of Engagement in the fall. These expectations are based both on the coping trend information noted by previous researchers (Fields \& Prinz, 1997; Skinner \& Zimmer-Gembeck, 2007; Zimmer-Gembeck \& Skinner, 2011), and the results obtained by this researcher in her unpublished Master's thesis study, wherein no prediction was found for the mastery ways of coping, 
but helplessness ways of coping did predict changes in subsequent Engagement.

Engagement predicting coping. It is expected that for all age groups, Engagement in the fall will positively predict mastery coping, and negatively predict helplessness coping in the spring. Additionally, the influence of Engagement on mastery ways of coping and for Confusion coping is expected to be stronger for older children, while the influence of Engagement on Escape coping is expected to be stronger for younger children. These expectations follow the same pattern of coping trends identified by Fields \& Prinz (1997) and Skinner \& Zimmer-Gembeck, (2011).

\section{1b) Coping and Achievement.}

Coping predicting achievement. It is expected that InformationSeeking coping in the spring will be positively associated, and Escape coping at both time points will be negatively associated with Achievement for all grades, but the influence of Information-Seeking on Achievement will be stronger for older children, and the influence of Escape coping on Achievement will be stronger for younger children, reflecting the ways of coping noted to be most prevalent in each age group.

Achievement predicting coping. Achievement is expected to predict only Escape coping, and this relationship is expected to be significant only for younger children. Escape coping is a coping strategy that is more often used 
by younger children (Fields \& Prinz, 1997; Skinner \& Zimmer-Gembeck, 2007; Zimmer-Gembeck \& Skinner, 2011), and in this researcher's unpublished Master's thesis study (Greene, 2007), was the only way of coping predicted by Achievement.

1c) Engagement as a mediator in the relationship between coping and achievement.

Based on the findings previously obtained by this researcher that Engagement mediates the effects of Information-Seeking on Achievement, and Escape on Achievement, it is expected that Engagement will fully mediate the effects of these ways of coping on achievement for all ages.

\begin{tabular}{|l|l|}
\hline $\begin{array}{l}\text { Table } 2 \\
\text { Summary of hypothesized results for Research Question } 1\end{array}$ \\
\hline $\begin{array}{l}\text { Effects Stronger for ... } \\
\text { Older children }\end{array}$ & \multicolumn{1}{c|}{ Younger children } \\
\hline $\begin{array}{l}\text { Mastery coping } \rightarrow \text { Engagement: }(+) \\
\text { Confusion } \rightarrow \text { Engagement: }(-)\end{array}$ & Escape $\rightarrow$ Engagement : (-) \\
\hline \begin{tabular}{l} 
Confusion $\rightarrow \Delta$ Engagement: $(-)$ \\
\hline $\begin{array}{l}\text { Engagement } \rightarrow \text { Mastery coping: }(+) \\
\text { Engagement } \rightarrow \text { Confusion : }-\end{array}$
\end{tabular} & Engagement $\rightarrow$ Escape: $(-)$ \\
\hline $\begin{array}{l}\text { Information Seeking (Spring) } \rightarrow \\
\text { Achievement: }(+)\end{array}$ & $\begin{array}{l}\text { Escape (Fall \& Spring) } \rightarrow \\
\text { Achievement: }(-)\end{array}$ \\
\hline $\begin{array}{l}\text { No Age Differences: } \\
\text { Coping } \rightarrow \text { Engagement } \rightarrow \text { Achievement: Full mediation }\end{array}$ \\
\hline
\end{tabular}

Note: $(+)=$ positive relationship; (-) = negative relationship; $\Delta=$ change in; $\rightarrow=$ prediction 
2. Do the unique and predictive relationships between the components of perceived control and coping change with age?

Consistent with previous research, perceived control as a tri-partite construct is measured as the indicator of the strength of the competence system, which is generally accepted as the self-system most closely-related to academic outcomes. The general expectation is that some components of perceived control are significant predictors of coping efforts across all ages, accounting for unique amounts of variance in coping over and above the amount of variance accounted for by other components of control included in the model, while others are predictive only for specific age groups and for specific ways of coping. These patterns of prediction for each way of coping may vary in strength by age group from one way of coping to another as well.

To assist in identifying the differential contributions of the components of perceived control in predicting ways of coping, these hypotheses will first be examined using the Total Strategy and Total Capacity belief sets, which combine beliefs about all the causal categories. When one of these belief sets is found to be significantly related to a way of coping, the components comprising the total will be tested to determine which of the components is the strongest predictor.

Overview. In general it is expected that Total Strategy beliefs will be most strongly related to ways of coping for younger children, and Total Capacity beliefs will be most strongly related to ways of coping for older children, as found by 
previous research and given that internal control tends to increase with age (Skinner, et al., 1998; Nowicki \& Strickland, 1973; Weisz \& Stipek, 1982).

Hypotheses regarding age-related differences for the Total Strategy and Total Capacity beliefs relating to specific ways of coping are presented next, organized according to each way of coping. Specific hypotheses regarding the components of control are offered as follow-up analyses where there is an expectation that the total aggregate will show a significant relationship. These hypotheses have been formulated based on the pattern of relationships found by this researcher in her unpublished Master's thesis, and on findings regarding the development of perceived control reported by Skinner, et.al., (1998) as described in Part 2 of the literature review for the present study.

\section{Control predicting Problem-Solving.}

Total Strategy Beliefs are not expected to show any age differences in the their connection with Problem-Solving, but Total Capacity Beliefs will show stronger relationships for younger children than for older children, given the decreasing strength of capacity beliefs over time, and that perceived control overall tends to decline over time.

Individual Capacity Beliefs. Effort and Ability Capacity Beliefs are expected to be more strongly related to Problem-Solving for younger children than for older children, as the strength of these capacity beliefs tends to decline over time. Additionally, younger children tend to overestimate the amount of 
objective contingency between their actions and subsequent outcomes, contributing to high levels of capacity beliefs, particularly Effort and Ability. Control predicting Information-Seeking.

Only Total Capacity Beliefs are expected to be related to InformationSeeking coping, according to the same pattern as expected for the individual components, with the relationship being stronger for older children than for younger children.

Individual Capacity Beliefs. Even though capacity beliefs tend to decline over time, it is expected that each capacity belief in the set (Effort, Ability, Powerful Others, and Luck) will be related to Information-Seeking coping more strongly for older children than for younger children. This expectation is based on the tendency of older children to engage more frequently in this coping strategy, due to its relational nature and older children's increasing preference for adult input. Control predicting Escape.

Given the curvilinear pattern of normative age changes in external strategy beliefs, it is expected that Total Strategy beliefs will not show age-related differences in the relationship with Escape coping. Total Capacity beliefs, however, are expected to be more strongly related for younger children than for older children, given that capacity beliefs are stronger for younger children and decline over time. 
Individual Capacity Beliefs. Given that younger children are higher on capacity beliefs than older children and engage more frequently in avoidance behaviors, it is expected that each of the components of Total Capacity beliefs will be more strongly related to Escape coping for younger children than for older children.

\section{Control predicting Confusion.}

Both Total Strategy and Total Capacity belief sets are expected to be more strongly related to Confusion coping for older children than for younger children, based on the tendency of older children to exhibit Confusion coping more often than younger children.

\section{Individual Strategy Beliefs. As the Powerful Others Strategy Beliefs} construct was not previously found to be related to Confusion coping in this researcher's unpublished Master's thesis, this strategy belief is not expected to be related to Confusion coping at any age. The other four strategy beliefs, however, (Effort, Ability, Luck, and Unknown) are expected to be more strongly related to Confusion coping for older children than for younger children, as older children tend not only to have higher strategy beliefs in these areas, but also to engage in more cognitive forms of coping, such as Confusion coping, versus avoidance behaviors, like Escape. 
Individual Capacity Beliefs. Likewise, three of the four capacity beliefs (Effort, Ability, and Luck) are expected to be more strongly related to Confusion coping for older versus younger children.

\section{Control predicting changes in coping.}

In this researcher's unpublished Master's thesis study, only the control aggregates were used to account for additional variance in the ways of coping in the spring, over and above that which was accounted for by ways of coping in the fall. For each way of coping except Escape, the amount of additional variance accounted for was very small, ranging from 1 to 3 percent. In the case of Escape coping, that range was a little larger, from 4 to 7 percent. Given that there is so little variance to be accounted for in Problem-Solving, Information-Seeking, and Confusion, age differences in changes in these ways of coping may be difficult to detect. Having noted this, these hypotheses will be tested in the present study using a profile of control beliefs referred to as CONMAX, which is the combination of control beliefs that represents maximum control. This aggregate has shown to be a strong and robust predictor in previous studies and may carry enough power to detect very small age-related differences in the changes that occur in ways of coping over time.

No specific hypotheses are offered for Problem-Solving, InformationSeeking, and Confusion coping. For Escape coping, it is expected that the CONMAX aggregate will be negatively related to additional variance in 
Escape coping in the spring after controlling for Escape coping in the fall for younger children, but not for older children, due to the powerful effects of a maladaptive control profile on Escape coping noted by previous researchers as children get older.

\section{Table 3}

Summary of hypothesized results for Research Question 2

\begin{tabular}{|l|l|}
\hline $\begin{array}{c}\text { Effects Stronger for ... } \\
\text { Older children }\end{array}$ & \multicolumn{1}{c|}{ Younger children } \\
\hline $\begin{array}{l}\text { Total Capacity (Effort, Ability, Powerful } \\
\text { Others, Luck) } \rightarrow \\
\text { Information Seeking: (+) }\end{array}$ & $\begin{array}{l}\text { Total Capacity (Effort, Ability) } \\
\text { Problem Solving: (+) }\end{array}$ \\
\hline $\begin{array}{l}\text { Total Strategy (Effort, Ability, Luck, } \\
\text { Unknown) } \rightarrow \\
\text { Confusion: (+) } \\
\begin{array}{l}\text { Total Capacity (Effort, Ability, Luck) } \\
\text { Confusion: (-) }\end{array}\end{array}$ & $\begin{array}{l}\text { Total Capacity (Effort, Ability, } \\
\text { Powerful Others, Luck) } \\
\text { Escape: (-) }\end{array}$ \\
\hline
\end{tabular}

Note:(+) = positive relationship; $(-)=$ negative relationship; $\Delta=$ change in; $\rightarrow=$ prediction 
3. Do the unique and predictive relationships between support provided by the social context and coping change with age?

Using the Self-Systems Model of Motivational Development (SSMMD) as a framework, which provides for the effects of contributions from the social context, interactions with the teacher are hypothesized to either help or hinder a child in his or her mastery-related behaviors. Motivational support includes provision of structure, involvement, and autonomy support by teachers, consistent with previous research based on the SSMMD (Skinner \& Belmont, 1993). The SSMMD further suggests that this contribution from the social context is predictive of a child's coping strategies. The following hypotheses outline the expected age-related differences in the prediction of coping strategies by Teacher Support.

While perceived control is not tested in the present study as a mediator of this relationship, hypotheses are based largely on the direction of influence reported by previous research for the relationship between Teacher Support and components of perceived control, because very little previous research has been done exploring the age-related differences of the influence of Teacher Support on the selection of coping strategies on which to base these predictions.

Teacher Support related to ways of coping, concurrently. The relationship between Teacher Support and ways of coping is expected to show a different pattern of age-related differences for each way of coping. With respect to 
Problem-Solving, prediction is expected to be stronger for younger children, due to the impact of Teacher Support on external strategy beliefs as noted by Skinner et.al., (1998), and the expectation that these strategy beliefs have a strong influence on Problem-Solving for younger children.

The opposite pattern of age differences is expected for InformationSeeking. Given that Teacher Support has demonstrated an important influence on Capacity Beliefs for older children, and older children hold stronger beliefs than do younger children about the influence of Powerful Others on subsequent success, it is expected that Teacher Support will be a stronger predictor of Information-Seeking coping for older children.

This researcher's unpublished Master's thesis work highlighted how Escape coping may ultimately be a more detrimental response for older children than for younger children. Given the strong negative association between Teacher Support and Escape coping, it may be that the lack of intervention by the teacher in a child's Escape tendencies may have a negative reinforcing effect as children age. Based on the evidence suggesting a maladaptive control profile is particularly devastating for children over time, prediction for the influence of Teacher Support on Escape is expected to be stronger for older children than for younger children. The prediction of Confusion coping by Teacher Support is expected to follow the same pattern as for Escape coping, although the strength of the associations is expected to be somewhat weaker. 
Teacher Support predicting changes in ways of coping. Consistent with previous unpublished findings of this researcher's Master's work, Teacher Support is expected to predict changes in all ways of coping except Confusion, with higher levels of Teacher Support associated with increases in masteryoriented coping and decreases in Escape coping. However, these findings indicated prediction of a very small amount of change in coping from fall to spring $\left(\Delta \mathrm{R}^{2} \cong .01\right)$; hence, no age differences in the prediction of those changes in coping are expected. Based on the lack of association previously noted by for the relationship between Teacher Support and Confusion coping, no prediction for either age group is expected.

\begin{tabular}{|l|l|}
\hline $\begin{array}{l}\text { Table } 4 \\
\text { Summary of hypothesized results for Research Question } 3\end{array}$ \\
\hline $\begin{array}{l}\text { Effects Stronger for .... } \\
\text { Older children }\end{array}$ & \multicolumn{1}{c|}{ Younger children } \\
\hline & $\begin{array}{l}\text { Teacher Support } \rightarrow \\
\text { Problem Solving: }(+)\end{array}$ \\
\hline $\begin{array}{l}\text { Teacher Support } \rightarrow \\
\text { Information-Seeking: }(+)\end{array}$ & \\
\hline $\begin{array}{l}\text { Teacher Support } \rightarrow \\
\text { Escape \& Confusion: }(-) \\
\text { (weaker for Confusion) }\end{array}$ & \\
\hline $\begin{array}{l}\text { No Age Differences: } \\
\text { Teacher Support } \rightarrow \Delta \text { Coping }\end{array}$ & \\
\hline
\end{tabular}

Note: $(+)=$ positive relationship; $(-)=$ negative relationship $; \Delta=$ change in; $\rightarrow=$ prediction 
4. Do the reciprocal effects of coping and engagement on components of perceived control and teacher support differ by age?

Feedback effects. Previous analyses conducted for this researcher's unpublished Master's thesis indicated reciprocal effects of ways of coping and Engagement on the perceived control aggregates and Teacher Support, with higher levels of Engagement and mastery-oriented coping associated with increases in Teacher Support and perceptions of control over time, and lower levels of Engagement and higher levels of helplessness coping associated with decreases in Teacher Support and perceptions of control over time. Figure 11 presents a causal loop diagram to illustrate the dynamical feedback relationship between coping and engagement and perceived control and teacher support, with mastery and helplessness coping depicted separately. Both loops are reinforcing loops. One the left-hand side, increases in Engagement and mastery coping lead to increases in Teacher Support and Perceived Control, which subsequently strengthen both Engagement and mastery coping. On the right-hand side, increases in helplessness coping lead to decreases in Teacher Support and Perceived Control, which subsequently strengthens the helplessness coping response. 


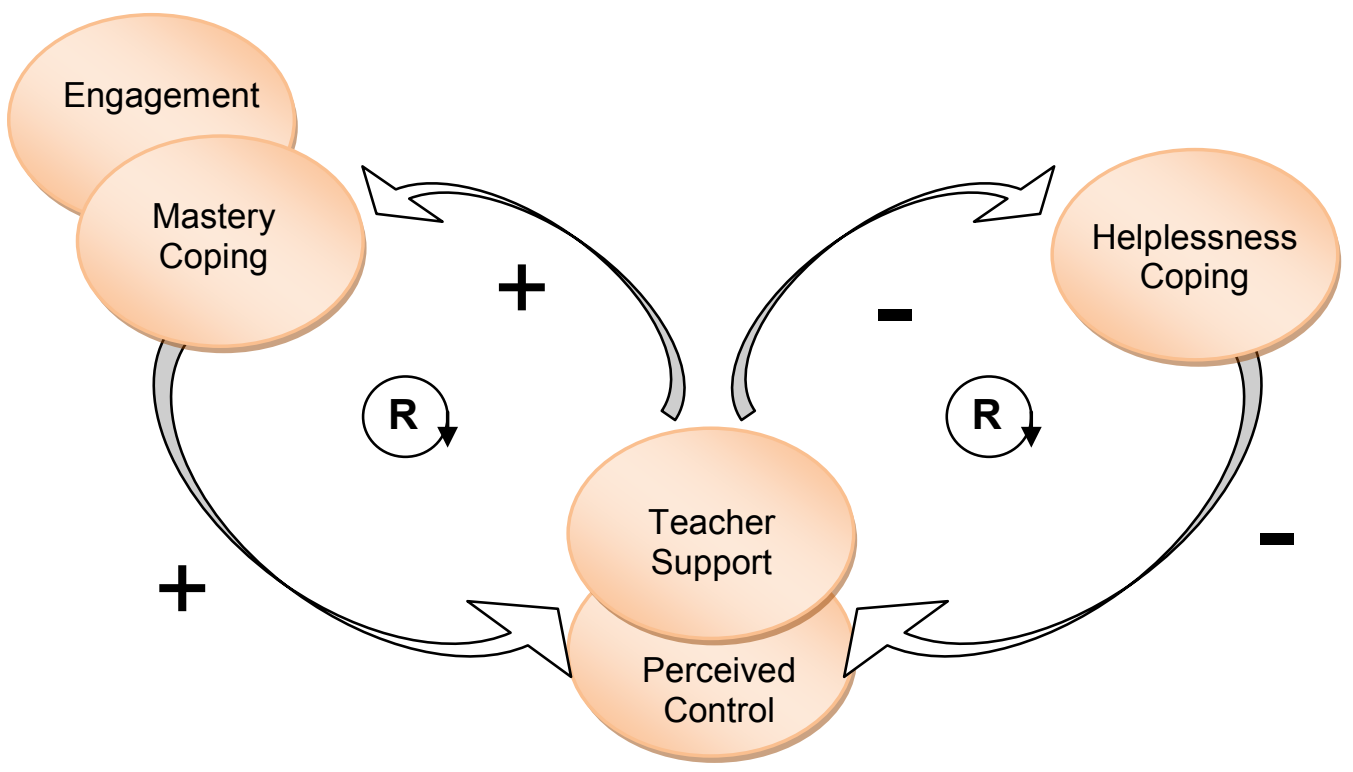

Figure 11: Causal loop diagram depicting dynamic feedback effects of ways of coping and engagement on Teacher Support and Perceived Control.

An evaluation of the age-related differences in these patterns is expected to yield results indicating stability of the general structure of these relationships across age groups. It is expected that cycles involving Engagement that demonstrate a positive feedback loop may be seen as children get older, as Engagement has been shown to increase over time until the transition to middle school. As children age, higher levels of Engagement are expected to facilitate increases in Teacher Support and control.

The feedback effects of coping on Teacher Support and control are expected to follow a differential pattern for each dependent variable. As children get older, increases in Teacher Support are expected with higher 
levels of the mastery ways of coping, and decreases are expected with higher levels of the helplessness ways of coping. For control, only higher levels of Information-Seeking will be related to increases in control as children get older. Problem-Solving was not related to changes in control and is not expected to demonstrate any age-related effects on changes in control. The helpless ways of coping are expected to demonstrate an inverse relationship to changes in control, with higher levels of the use of helplessness coping accounting for decreases in control as children get older.

If obtained, these results confirm the reciprocal effects model and provide evidence of amplification cycles of interaction between these constructs.

\section{Table 5}

Summary of hypothesized results for Research Question 4

Effects Are Stronger As Children Get Older...

Engagement $\rightarrow \Delta$ Teacher Support \& $\Delta$ Control: $(+)$

Mastery Coping $\rightarrow \Delta$ Teacher Support: $(+)$

Helpless Coping $\rightarrow \Delta$ Teacher Support: (-)

Information-Seeking $\rightarrow \Delta$ Control: (+)

Helpless Coping $\rightarrow \Delta$ Control: (-)

Note: $(+)=$ positive relationship; $(-)=$ negative relationship $; \Delta=$ change in;

$\rightarrow=$ prediction 


\section{Summary of Hypotheses}

\section{Age Differences in Relationships Between Coping, Engagement, and Achievement}

H1a1. Coping will predict Engagement, with stronger effects for:

- $6^{\text {th }}$ grade for:

- Mastery coping (positive prediction)

- Confusion coping (negative prediction)

- $4^{\text {th }}$ grade for:

- Escape coping (negative prediction)

H1a2. Only the helpless ways of coping will predict changes in Engagement, with stronger effects for:

- $6^{\text {th }}$ grade for:

○ Confusion coping (negative prediction)

- $4^{\text {th }}$ grade for:

○ Escape coping (negative prediction)

H1a3. Engagement will predict coping, with stronger effects for:

- $6^{\text {th }}$ grade for prediction of:

- Mastery coping (positive prediction)

- Confusion coping (negative prediction)

- $4^{\text {th }}$ grade for prediction of:

$\circ$ Escape coping (negative prediction)

H1b1. Information-Seeking and Escape coping will predict Achievement, with stronger effects for:

- $6^{\text {th }}$ grade for:

$\circ$ Information-Seeking in the spring (positive prediction)

- $4^{\text {th }}$ grade for:

$\circ$ Escape coping in the fall and spring (negative prediction)

H1b2. Achievement will have reciprocal effects only on Escape coping, with stronger effects for $4^{\text {th }}$ graders (negative association).

H1c1. Engagement will fully mediate the effects of ways of coping on Achievement, with no age differences detected.

Table 6: Summary of Hypotheses. 


\section{Summary of Hypotheses}

\section{Age Differences in the Effects of Perceived Control on Coping}

H2a. Control belief sets will predict Problem-Solving, with no age differences for:

- Total Strategy Beliefs predicting Problem-Solving (negative prediction) and stronger effects for:

- $4^{\text {th }}$ grade for:

- Total Capacity Beliefs predicting Problem-Solving (positive prediction)

- Capacity components of Effort and Ability significantly related

$\mathrm{H} 2 \mathrm{~b}$. Control belief sets will predict Information-Seeking, with no age differences for:

- Total Strategy Beliefs predicting Information-Seeking (negative prediction)

and stronger effects for:

- $6^{\text {th }}$ grade for:

○ Total Capacity Beliefs predicting Information-Seeking (positive prediction)

○ All Capacity components significantly related

H2c. Control belief sets will predict Escape, with no age differences for:

- Total Strategy Beliefs predicting Escape (positive prediction) and stronger effects for:

- $4^{\text {th }}$ grade for:

- Total Capacity Beliefs predicting Escape (negative prediction)

- All Capacity components significantly related

H2d. Control belief sets will predict Confusion, with stronger effects for:

- $6^{\text {th }}$ grade for:

- Total Strategy Beliefs predicting Confusion (positive prediction)

- All Strategy components except Powerful Others significantly related

- Total Capacity Beliefs predicting Confusion (negative prediction)

- All Capacity components except Powerful Others significantly related

H2e. Maximum control (CONMAX) will negatively predict changes in Escape for $4^{\text {th }}$ grade only. No prediction for $6^{\text {th }}$ grade is expected. 


\section{Summary of Hypotheses}

\section{Age Differences in the Effects of Teacher Support on Ways of Coping}

H3a. Teacher Support will positively predict Problem-Solving, with stronger effects for $4^{\text {th }}$ grade.

H3b. Teacher Support will positively predict Information-Seeking, with stronger effects for $6^{\text {th }}$ grade.

H3c. Teacher Support will negatively predict Escape and Confusion, with stronger effects for $6^{\text {th }}$ grade, but the association for Confusion will be weaker than for Escape.

H3d. No age differences will be found in the prediction of changes in any way of coping by Teacher Support.

\section{Age Differences in the Feedback Effects of Children's Coping and Engagement on Teacher Support and Perceived Control}

H4a. Engagement will feed back onto Teacher Support and control beliefs, with stronger effects for $6^{\text {th }}$ grade.

H4b. Coping will feed back onto Teacher Support, with effects stronger for $6^{\text {th }}$ grade.

H4c. All ways of coping except Problem-Solving will feed back onto control beliefs, with effects stronger for $6^{\text {th }}$ grade.

Table 6: Summary of Hypotheses, cont'd. 


\section{CHAPTER 4: METHODS}

Participants. Data for this study were obtained from a four-year longitudinal study on children's coping and motivation in the classroom conducted in a ruralsuburban school district in upstate New York. Data were collected every fall (October) and spring (May) of the academic year for three consecutive years. All students in the school district attended one school. Elementary students were taught in traditional self-contained classrooms; middle school began with $6^{\text {th }}$ grade, where students were with homeroom teachers for a significant portion of the day.

During the first year of data collection, children in grades 3 through 5 and their teachers participated. These children were followed in year 2, who were then in grades 4 through 6 , and a second wave of third graders was recruited. Additional $4^{\text {th }}$ and $5^{\text {th }}$ graders who had not previously participated but whose teachers became willing to participate were also recruited. As a result, year 2 data were collected from the wave 1 students, who were then in grades 4 through 6 , and the wave 2 students, who were in grades 3 through 5 . In year 3, data collection was conducted with both waves of students, with a grade span of grades 4 through 7 .

The fall and spring measurement points from year 2 were chosen for this study because this year provides the most complete data for the coping variables. During year 2, data were collected from approximately 900 children in grades 3 through 6 and 53 of their teachers. Table 7 indicates the specific numbers of students surveyed by grade at each time point. The sample was nearly equally divided by gender and was 
predominantly Caucasian, with less than 5\% identifying as non-white. Parents' socioeconomic status, determined by level of education and occupation, ranged between lower-middle and middle class.

\section{Table 7}

Number of Students Assessed at Each Grade Level at Each Measurement Point for Year 2

\begin{tabular}{|c|c|c|}
\hline \multirow{2}{*}{ Student Grade } & \multicolumn{2}{|c|}{$\underline{\text { Year } 2}$} \\
\hline & Fall & Spring \\
\hline 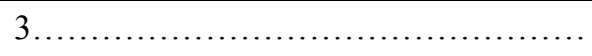 & 132 & 118 \\
\hline …................... & 333 & 318 \\
\hline ............ & 161 & 148 \\
\hline $6 \ldots \ldots \ldots \ldots$ & 342 & 312 \\
\hline Total & 968 & 896 \\
\hline
\end{tabular}

Developmentally, the transition to middle school in $6^{\text {th }}$ grade is accompanied by notable normative shifts in both physiological and psychological processes. Because this study seeks to identify age differences in strategies for coping and perceptions of control, age comparison analyses should compare a younger group against the students in the $6^{\text {th }}$ grade group. Balanced age groups are desirable for these analyses, and so this study used the $4^{\text {th }}$ grade group as the younger age group and the $6^{\text {th }}$ grade group as the older age group, excluding the $3^{\text {rd }}$ and $5^{\text {th }}$ grade students. The groups used for this study are indicated in Table 7 with the bolded boxes around the number of subjects. This yields a preliminary data set of 675 students.

Attrition. Given that students were surveyed at more than one time point using the same instrument, an opportunity for the introduction of positive bias to the data 
existed. An analysis using a 2 X 2 MANOVA model (a 2 between [grade groups: 4, 6] by 2 within [attendance status: present in spring, not present] model) was performed on 8 scales to determine whether there are any significant differences in the means of those scale scores between students who provided data in both the fall and spring, and those who provided data only in the fall. The eight scales chosen for this analysis included the two control aggregate measures (Total Strategy Beliefs and Total Capacity Beliefs), all four coping scales, the aggregate engagement scale, and the aggregate teacher support scale. The aggregate scales were used instead of the individual scales to minimize the test-wise error rate.

All 675 students provided at least some data in the fall, and twenty-two $4^{\text {th }}$ grade students and fifty-four $6^{\text {th }}$ grade students were either absent or did not respond at all in the spring. The eight scales used as the dependent variables for this analysis were examined to determine whether the assumptions of MANOVA would be met with respect to distributional properties. All scales were found to largely conform to a normal distribution and be within acceptable limits (skewness statistic less than 1.00, kurtosis statistic less than 2.5, histograms relatively symetric). Box's M test for homogeneity of covariances was significant $(\mathrm{p}=.011)$, indicating the protection against a Type I error normally afforded by the MANOVA procedure may not extend to the univariate tests should the multivariate test show a significant main effect for any of the independent variables. Tabachnick \& Fidell (1996) note that Box's M test is highly sensitive to tiny differences; when sample sizes are large and cell sizes are 
equal, it can safely be ignored. Cell sizes are not equal in this analysis, however, so an alpha level of $.006(.05 / 8$ DVs) will be used to protect against a Type 1 error. Levene's test of homogeneity of variance was not significant for any variable, indicating the conditions for the MANOVA test with respect to homogeneity of variances was met.

As another strategy to guard against inflated alpha error rates, Pillai's criterion, which is more robust than the Wilk's criterion, was used to evaluate the omnibus test, and was significant for the main effect of grade, Pillai's $\Lambda=.06, F=2.741,(8,330)$, $\mathrm{p}<.01$, parital $\eta^{2}=.06$, but was not significant for the main effects of attendance status, Pillai's $\Lambda=.041, F=1.714,(8,330), \mathrm{p}<.09$, parital $\eta^{2}=.04$, or the grade * attendance interaction, Pilla's $\Lambda=.041, F=1.017,(8,330), \mathrm{p}<.42$, parital $\eta^{2}=.02$. Power to detect the effect of grade was .935; to detect the effect of attendance, power was .752 , and power to detect the effect of the grade * attendance interaction was .474 .

Standard procedure when a significant main effect is found for an IV is to interpret the between-subjects univariate tests to further understand the multivariate result. However, grade differences are the outcome of interest of the full study, not the attrition analysis, and a significant main effect of grade provides no additional useful information in the consideration of the effects of attrition. The IV of interest for the attrition analysis is attendance status, and main effects for neither attendance status nor grade * attendance status were found. Based on this finding, the univariate 
results are not interpreted. The conclusion regarding the effects of attrition on differences in scale scores is that the responses of students who responded in the fall only do not differ significantly from those of students who responded in both the fall and spring.

\section{Treatment of Missing Data}

Mechanisms of missingness. Any longitudinal study is most likely plagued by the condition of incompleteness, in that some data for some participants at one or more time points may be missing. Decisions about how to address this condition in subsequent analyses must be made to ensure the integrity of the study and that bias in the resulting population estimates is minimized. The first consideration in addressing the condition of missing data is to determine the nature of the missingness. Three mechanisms that can produce missing data that are commonly described by practitioners are 1) missing completely at random (MCAR), 2) missing at random (MAR), and 3) missing not at random, (MNAR).

Missing data that is MCAR can be thought of as being missing due purely to chance. More precisely, data are MCAR if the probability of a missing response is independent of all measured and unmeasured characteristics of the study participants; the missing data is not related to either observed variables, or unobserved variables. Data are rarely MCAR, especially in longitudinal studies.

Data that are MAR may be missing due to planned features of the study design (e.g., not surveying particular groups at every time point), or may be missing 
depending on other observed characteristics of the study participants, but not on some underlying characteristic of the missing variables themselves. For example, a study that relates academic performance to a set of variables that includes income and prior educational attainment may have a large percentage of data missing for the income variable. If less educated individuals tend not to report their income, then whether income is missing or not may be dependent on level of education, as captured by the prior educational attainment variable, not on the income variable itself, and the mechanism of missingness is considered MAR. If however, the tendency to report income is dependent on level of income (high or low), then whether income data is missing or not depends on an underlying characteristic of income itself, and this pattern of missingness would fall into the third category of mechanisms of missingness, MNAR.

Data that are MNAR, or that are non-ignorable, are related to the value that would have been observed had it been reported. All types of missing data are problematic and can lead to biased estimates of the true population parameters if not properly accounted for. MNAR data is particularly problematic, however, given that under this mechanism the data that are missing are related to the underlying values of the variables of interest and are unknowable, and there are currently few accessible statistical means to correct for this condition. The current procedures being used to address the MNAR condition are complicated and involve constructing a model of the missingness mechanism, which is subject to misspecification and carries no guarantee 
that the resulting estimates will not still be biased, or any more precise than those obtained through procedures used under MAR conditions, even when data are MNAR.

When data are MCAR or MAR, robust statistical procedures have been identified, such as maximum - likelihood estimation (ML), using an expectationmaximization (EM) algorithm, or multiple imputation (MI), all of which provide adequate correction to sample estimates that minimize bias in estimates of population parameters (Graham \& Schafer, 1999; Schafer \& Graham, 2002; Graham, 2009). Missing values analysis. In the current study, because student participation was based on attendance (students who were present on the day the questionnaires were administered participated), missing data is expected to be at least missing at random (MAR). However, while there are simple procedures available to test the assumption that data are MCAR, there is no concrete method of distinguishing between data that is MAR and data that is MNAR. Missing data experts point out that because data that are MCAR are rare, and that the assumption that data are MAR versus MNAR is untestable, the goal of any missing values analysis should focus not on labeling the data as one type versus another, but on determining whether there exists a violation of MAR status big enough to threaten the validity of the study (Graham, 2009).

Under this advice, a careful examination of the data to arrive at a reasoned conclusion regarding the existence and size of this violation was undertaken and included assessing the maximum percentage of missing data for each data source 
(student, 24\%, teacher, 11\%, achievement indicators, 64\%), examining graphical patterns of missing data, and examining differences in parameter estimates between variables in differing patterns of missingness, e.g., variable pairs where one variable is missing versus present, and scale scores where complete cases exist versus partial cases versus cases with imputed values obtained via EM procedures, according to the recommendations of Graham (2009). Based on the small numbers of instances where parameter estimates significantly differed according to missingness patterns, in conjunction with the non-significant findings of the attrition analysis, and reassured by the simulation research that demonstrates erroneous acceptance of the MAR assumption often results in only minor impact on parameter estimates (Collins, Shafer \& Kam, 2001; Demirtas, Freels \& Yucel, 2008), a determination was made that there is insufficient evidence to suggest that the potential violation of the MAR assumption for this study is large enough to invalidate treatment of the data as MAR. Subsequent to this conclusion, missing data could be imputed via EM or MI methods, according to the recommendations of Schafer and Graham (2002). This lead to a new set of decisions to consider, beginning with a decision as to which method to use.

Methods of data imputation. Schafer and Graham (2002) published a review of missing data procedures in which they evaluated the results and limitations of a variety of techniques, both old and relatively new. After a thorough review of procedures ranging from list-wise and pair-wise deletion, to mean replacement, to single-regression imputation, and ending with simulation studies of both ML 
estimation and MI procedures, they strongly recommended that researchers apply either ML estimation or MI procedures, as these methods provide the least biased estimates and are currently considered by missing data experts as state of the art techniques. Note that in ML estimation using EM procedures, a single imputed dataset is produced, whereas with MI, multiple complete datasets of plausible value estimates is the end product. The data set produced by the EM procedure is a complete-case dataset, and can be analyzed using any popular statistics package, like any other dataset would be. The multiple datasets produced by the MI procedure can also be analyzed with any popular statistics package, just like the EM dataset, but analyses are run on all imputed datasets individually, and the results for each dataset pooled to arrive at a single set of results for the analysis. Computations and rules for pooling estimates from MI datasets were specified by Rubin (1987), and are referred to by practitioners as "Rubin's Rules."

In a subsequent review article, Graham (2009) offered a contrast of the two procedures, and recommendations as to the conditions under which one method is more appropriate than the other. He states that the best choice for missing data treatment accomplishes three things: 1) properly accounts for uncertainty introduced by missing values, 2) produces appropriate standard errors so as to minimize bias, and 3) preserves the sample size and prevents loss of information.

In his discussion of ML estimation and the EM algorithms that produce missing value and parameter estimates, Graham (2009) points out that the key to 
unbiased imputation is restoring the variance lost in regression-based single imputation. The EM algorithms for ML estimation add back an error term to the value estimates, but all iterations are based on a single initial draw from the population of interest (the original data), and yield standard errors that are too small. Moreover, those standard errors are typically not readily available as output, making further analysis and error corrections to imputed values difficult. For this reason, Graham (2009) cautions that a single imputed data set from an EM procedure is not a good choice for analyses involving hypothesis testing. Rather, he recommends that single imputed EM datasets be used only for parameter reporting (e.g., means, standard deviations, correlations), or data quality exploration, such as calculation of coefficient alpha, exploratory factor analysis, or other analyses that do not rely on standard errors.

In contrast, MI simulates multiple random draws from the population through a process known as data augmentation (DA; Tanner \& Wong, 1987). Data augmentation allows for simulation of multiple draws from the population and imputation of multiple complete datasets, of which only every $n t h$ dataset will be retained, to ensure that the datasets that are retained approximate random draws as closely as possible. Data augmentation is a member of the Markov-Chain Monte Carlo (MCMC) family, and as such, all the information from one step of DA is contained in the previous step, making parameter estimates from two consecutive draws more similar than would be expected by chance. Thus, the farther away from each other two draws are, the more dissimilar they will be, and thereby better 
approximate a random nature. This feature of MI produces larger standard errors than are produced by the EM algorithms of ML estimation, which is more suitable for use in hypothesis testing (Graham, 2009). For additional detail on the DA procedure, see Graham, Cumsille, \& Elek-Fisk (2003).

While both ML estimation via EM procedures and MI techniques offer the distinct advantage of preserving the relational properties between variables, there is a very important difference between the output that each produce, grounded in the difference in how each procedure defines convergence. In ML estimation using an EM algorithm, convergence occurs when the differences in the parameter estimates from one iteration to the next stabilize and change so little from iteration to iteration that they are said to have converged to a single set of values. In MI, because new sample draws are made at each iteration, from which parameters are estimated, the parameters themselves never converge to a single set. In MI, convergence occurs when the distribution of parameters stabilizes and yields an essentially unchanging set of values from one iteration to the next, after which retention of datasets begins. The benefit of this is that the imputed missing values themselves are not important; they can be values that are not necessarily consistent with the original metric of the variables, because the distributional properties of the original dataset are preserved and reflected in each of the retained datasets, thereby reducing bias and increasing power (Peugh \& Enders, 2004). Another important point in comparing ML estimation to MI methods is that MI assumes multivariate normality. To test this assumption on 
the data for the current study, Small's omnibus test of multivariate normality (Small, 1980) was performed on the dataset that was specified for the imputation model (as described in sections below). The test was significant, $\chi^{2}=4607.733 d f=644$, $\mathrm{p}=.000$, indicating the dataset is not multivariate normal. However, simulation studies conducted by multiple teams of missing data researchers (Schafer \& Graham, 1999; Collins, et al., 2001; Demirtas, et al., 2008) offer ample evidence that MI results obtained under substantial violations of this assumption are still quite robust with a minimum level of bias.

Imputation of missing values. Given that the analyses for the present study rely heavily on multiple regression for hypothesis testing, the decision was made to use MI to impute the datasets for analysis, even though the test of multivariate normality was significant. This finding should be considered when interpreting the results of subsequent analyses, as the results may be attenuated. A single-imputation dataset obtained via EM procedures during the missing values analysis was used for reporting of descriptive statistics and coefficient alphas.

The next set of decisions included specification of the imputation model, and determination of how many datasets to retain. Specifying an imputation model that differs from the analytic model is not an explicit option in ML estimation techniques such as EM procedures or full information maximum-likelhood estimation (FIML) because missing data handling and the analysis model are addressed at the same time during each iteration of the process, and most researchers include only those variables 
that will also appear in subsequent analyses to avoid biasing estimates with information from auxiliary variables (Peugh \& Enders, 2004). In contrast, however, with MI one can specify an imputation model that differs from the analytic model to increase power and reduce bias because the procedure is theoretically a Bayesian procedure that utilizes a two-step process during each iteration that holds distinct the imputation phase from the analytic phase. In Bayesian terms, the first step of each iteration is based on the prior distribution of observed variables (outcome of the previous iteration), and the second step of the iteration is based on the posterior dataset of predicted values, (the one obtained in the first step of the current iteration), thus keeping the computations for the imputation phase separate from the analytic phase (Peugh \& Enders, 2004). This feature allows for inclusion in the imputation model of variables that have predictive power for the missing values because of their correlation with the variables being imputed, but which will not be included in subsequent analyses, without compromising parameter estimates. Hence, the question of what to include and what not to include for the imputation process in MI must be given explicit consideration.

Specifying the imputation model. Graham (2012) offers three guiding principles when specification of the imputation model is being considered. He advises researchers to 1) include variables related to the variables being imputed, 2) include variables potentially related to missingness, and 3) ensure the imputation model is at least as general as the analysis model. It is the third principle that merits the most 
careful consideration for the present study. In plain words, this principle translates to the requirement that all variables in the analysis model be included in the imputation model, which raises the question then of how to account for interaction terms in the imputation model. Each research question under investigation in this study includes an interaction term, so careful consideration of this issue is warranted. At first glance, the answer seems simple - just calculate the interaction terms before imputation and include them in the imputation model. Unfortunately, there are more aspects of this issue to be taken into account.

Graham (2009) addresses the issue of computational performance, and points out that as the number of variables to be imputed increases, the number of computations required by the imputation process increases exponentially. Very quickly an imputation project can get bogged down, take hours to complete, or may never converge. To guard against this, Graham (2009) recommends limiting the number of variables to be imputed to less than 100 for large datasets $(n=1000)$, fewer for smaller datasets $(n=50)$. The dataset for the present study, at the item level, contains over 300 variables, with $n=665$, before calculating scales and interaction terms. Graham (2009) offers several alternatives to reduce the number of variables being imputed and improve computational performance, while preserving the statistical efficiency of the resulting estimates. Of his suggestions, the most feasible for the present study was to impute whole scales. To do this, he recommends that all cases have scales scores that are computed from complete data for the scale, and 
where only partial data on a scale is available, the scale score be left as missing to be imputed. Schafer and Graham (2002) suggest that scale scores based on partial data may be acceptable in some situations, but can cause problems in others. Graham (2009) does list the conditions under which scale scores calculated from partial data are acceptable, none of which are tenable for the present study data. Consequently, all instances of partial data for each scale were discarded before calculating scale scores. After scale calculations were performed, the number of scale variables to be imputed was 71 , and the number of achievement variables to be imputed numbered 14 . The number of interaction terms to be computed was 48 , meaning the total number of variables to be imputed was still greater than the recommended 100 .

The second strategy used to reduce the overall number of variables to impute resulted from the recommendations Graham $(2009 ; 2012)$ made regarding treatment of the interaction terms in the imputation model, as described next.

Accounting for interactions during imputation. An interaction term is the product of two other variables, and as such, represents a non-linear combination of those variables. The imputation model is a linear model, and the resulting parameter estimates for the imputed values of a non-linear term will not be consistent with the estimates for each of the component variables of the product term. The alternative, which is to calculate the interaction terms after imputation, also does not provide accurate estimates because the imputation process assumes that the correlation between the omitted interaction term and all other variables in the imputation model is 
equal to zero. The consequence of this situation then is that the relationship between the interaction term and the DV in an analysis will be attenuated towards zero, and this is not a desirable outcome. Until recently, the best-case scenario agreed upon by missing data experts has been to include the interaction term in the imputation model and use the resulting values in subsequent analyses, in spite of the known issues. VonHippel (2009) was able to demonstrate that this approach, while not ideal, did still produce reasonably efficient population estimates.

VonHippel's work (2009) was extended by Enders, Baraldi, \& Cham (2014) to document a variety of centering techniques that successfully correct for the loss of efficiency previously noted in population estimates produced from analyses using imputed interaction terms.

It is important at this point to note that these issues and solutions have been documented to apply to interaction terms involving two continuous variables. The solutions offered by Graham $(2009 ; 2012)$ apply to interaction terms where one of the variables is a categorical variable; none of the issues mentioned previously affect interaction terms that are the product of a continuous and a categorical variable. In the present study, all interaction terms are the product of a continuous variable and grade in school, which has two levels, $4^{\text {th }}$ grade and $6^{\text {th }}$ grade. The grade variable is used as a theoretical proxy for age in interpreting the results, but is not a continuous variable, as a true measure of chronological age would be. 
Graham $(2009 ; 2012)$ suggests that in order to ensure that any interaction term that might be of interest in the analytic model is accounted for by the imputation model where those interaction terms are the product of a continuous and a categorical variable, one should impute the dataset separately for each level of the categorical variable. To not do this would be making the assumption that the correlation with other variables is the same for all levels of the variable. This is exactly the variation the present study is attempting to detect, so safeguarding this variance is of great importance. By imputing scale score data separately for each grade, the correlations for each level of the categorical variable for grade are preserved. The added benefit for the imputation process is that the need to compute the interaction terms prior to imputation is eliminated, thereby reducing the number of variables to be imputed. Based on this advice, the decision was made to impute a dataset for each grade separately. Treatment of the achievement variables was handled differently, as described in the next section, and so those variables were excluded from the imputation model for these datasets, leaving 71 variables in the imputation model.

Treatment of missing achievement scores. In the analysis model, the achievement scores were to be used to calculate a single achievement index, and would appear as both a DV and an IV in only one set of analyses. The amount of missing data in these variables was considerably higher than for the student- or teacher- reported data. Preliminary imputation runs demonstrated that the large amount of missing data caused many negative and other out of range values to be 
imputed. For ease of calculation of the achievement index and subsequent interpretation, it was desirable to have these variables constrained in the imputation model to the original scale of the variables. This constraint proved problematic for convergence of the procedure when these variables were included with the full complement of scale scores. Further, the $4^{\text {th }}$ grade students were not expected to have the same number of achievement scores as the $6^{\text {th }}$ graders (the $6^{\text {th }}$ graders had two additional years of testing recorded), so imputations on some of the achievement variables were not needed for the $4^{\text {th }}$ grade students.

To address these difficulties and concerns, the decision was made to impute the achievement variables separately for each grade, using the variables that would appear in the analysis model, as well as any other variable that correlated with achievement at a level of $r=.50$ or better (Graham, 2009). This further reduced the number of variables included in the imputation model for the scale scores. A total of 24 variables were included in the imputation model for the $4^{\text {th }}$ grade students, and 30 variables were included in the imputation model for the $6^{\text {th }}$ grade students.

Number of imputations. The last decision to be made concerned how many datasets to impute. Early work with MI procedures led experts to recommend that as few as only three to five datasets were needed to achieve adequate efficiency of the resulting parameter estimates. This was at a historical time when computing power was still developing, and computational performance was a driving factor in how widespread a procedure could be used in real practice. If only five or fewer datasets 
were needed to use MI procedures, then there was a better chance that more researchers would spend the time and effort to employ it. Rapid advances in computing technology over the past two decades, as well as the development of procedures within popular software packages for handling missing data has led to wider availability and accessibility of these techniques, and reintroduced the question of how many datasets really are necessary to impute. While efficiency of parameter estimates was the primary concern in early work, the general emphasis on statistical power that has emerged over the past decade has caused experts to pick up this question again, this time from the standpoint of whether the original recommendation of three to five datasets also provides adequate statistical power to detect small effects $(\rho=.10)$.

Simulation studies by Graham, Olchowski, and Gilreath (2007) have demonstrated that compared to results from analyses conducted with data imputed via FIML procedures, there is a substantial reduction in power with fewer datasets. To maintain statistical power to detect a small effect size at the same level as an equivalent FIML model, Graham et.al, (2007) suggest that with 50\% missing information, a minimum of 40 imputed datasets are needed in MI. Graham (2012) offers a table from which to calculate the number of datasets necessary based on the amount of missing information in the original dataset in order to maintain sufficient statistical power to detect an effect size as small as $(\rho=.10)$. Preliminary analyses during the missing value analysis for the present study indicated that the percent of 
missing data noted equates to a rate of missing information for the overall dataset of approximately $30 \%$. According to the table provided by Graham (2012), it is recommended that a minimum of 20 datasets be imputed.

Given that the percent of achievement variables that were missing was considerably higher $(\max =94 \%)$, the decision was made to adjust the recommended minimum of 20 imputed datasets by doubling it to 40 . It is difficult to determine the true extent to which this was necessary, but the additional computational effort needed to pool the results of 40 datasets versus 20 during the analysis phase is negligible, and error on the side of caution is rarely regrettable.

Final imputation procedures. A check to see if all three of the principles for specifying an imputation model laid out by Graham (2009) had been met indicated that in fact they had, and imputation could proceed. In review, those principles recommend that researchers 1) include variables related to the variables being imputed, 2) include variables potentially related to missingness, and 3) ensure the imputation model is at least as general as the analysis model. Because the analytic models used in the present study encompass testing of reciprocal relationships, and all variables are both IVs and DVs, the first two principles were satisfied first by including all student- and teacher- reported variables in the imputation model for the scale scores, and second by including the analytic and correlated variables in the imputation model for the achievement scores. The third principle was met by 
accounting for the interaction terms according to established recommendations (Graham, 2009; 2012), as previously described.

The steps taken to obtain the final datasets for analyses were as follows:

First, all partial scale score data was discarded, and scale scores were calculated.

Next, data were separated by grade, and then two datasets were created for each grade.

The first data set contained only the scale score variables. The second dataset

contained the achievement variables, the coping variables, and the engagement

variables. The values for the coping and engagement variables were taken from the single-imputation dataset of complete values obtained via the EM procedure. Using the missing values module in SPSS 21, the imputation procedure was conducted for all four of these datasets, with 40 imputed datasets resulting for each. The achievement variables only were taken from the achievement datasets and combined with the scale score datasets, which were then collapsed into one complete data file containing the 40 imputed datasets for both grades. Descriptive statistics were run for all scale variables and compared to those of the original dataset, and were found to be essentially the same. The final steps in preparing for the study analyses included decisions about centering the predictor variables, and then computation of the interaction terms.

Pooled results. Because a number of individual datasets are imputed and subsequent analyses are conducted on each dataset, the analysis results from each dataset must be combined in some fashion to yield one set of interpretable results. Guidance known as "Rubin's Rules" (Rubin, 1987) is the authoritative word in the 
field on performing these aggregations. In general, most results can simply be averaged. SPSS provides pooled estimates for many different analyses for both averaged results and other results that require additional calculations. SPSS does not, however, provide pooled standardized regression coefficients, only unstandardized coefficients. Where standardized coefficients are desired, standardized versions of the variables used as IVs were used so that the pooled unstandardized coefficients provided in the SPSS output would be equivalent to the standardized coefficients. Because standardized variables were used, there was no need to also center the variables, as was originally planned. Pooled estimates for F-test statistics are also not given, and no guidance is provided by Rubin on this. Common practice in the field appears to be to report the range of F-statistics obtained from all imputed datasets, and this practice has been adopted for the current study.

Because multiple imputation has been chosen as the preferred treatment for missing data, the issue of pooled estimates presents a problem for the analyses planned for testing the mediational model proposed in Research Question 1. In a later section of this dissertation that addresses the analytic models used in this study, an in-depth discussion of recent advancements in the area of testing mediator / moderator models is presented, outlining the methodology used for this specific analysis that allows for pooled estimates to be obtained. 


\section{Data Collection}

Procedure. Self-report questionnaires were administered to all students by trained interviewers in their classrooms over three 40-minute sessions. During each session, an interviewer read the questions out loud while the students recorded their responses on the questionnaire. A second interviewer was available to monitor comprehension and field questions from the students. Teachers were not present in the classroom during these sessions; most filled out their own questionnaires in a separate room.

Measures. Students responded to a variety of items regarding ways of coping, aspects of their perceptions of control, and their own levels of engagement in the classroom. Teachers reported on the level of involvement, structure, and autonomy support they provided each child in the classroom. Each child was assessed by the teacher who felt they knew him or her best. Grades were collected from student records for a subset of the participants. All questionnaire items are included in Appendix A.

Response options were given on a forced choice, 4-point Likert type scale for each item, meaning students could choose one and only one response for each item. Available responses were: Not at all true (1), Not very true (2), Sort of true (3), or Very true (4). All scales used in this study except the scales for the ways of coping contained both positively and negatively worded items. Negatively worded items were then reverse coded. The items for each scale were averaged together to form a 
composite score, ranging from 1 to 4 . Higher scores indicated stronger endorsement of the construct.

Student Report Scales

Ways of Coping. Multiple ways of coping were assessed corresponding to each of the three self-systems. The present study uses only those ways of coping relevant to the competence system, i.e., Problem-Solving, Information-Seeking, Escape, and Confusion. The items take one of two forms: either a stem is presented (e.g., When I have trouble with a subject in school...) followed by a number of responses for which the student indicates his/ her level of endorsement, or a simple statement reflecting the construct is presented for endorsement.

Five items measure Problem-solving coping, and include items such as, "I try to see what I did wrong." Information-seeking is measured by five items, including items such as, "I ask the teacher to go over it with me." Four items are used to measure Escape coping, including items such as, "If a problem is really hard, I just quit working on it." Confusion coping is measured by five items, including items such as, "I'm not sure what to do next." The stems and full list of responses and statements for each scale used in this study are presented in Appendix A.

In this researcher's unpublished Master's thesis, the psychometric properties of the coping scales were examined and found to be distinguishable, uni-dimensional scales, with satisfactory factor loadings for each item in the scale $(\geq .50)$ obtained in confirmatory factor analyses conducted for both fall and spring measurement points. 
Reliabilities for each coping scale were also adequate (Problem-Solving: $\alpha=.68$, Information-Seeking: $\alpha=.72$, Escape: $\alpha=.77$, Confusion $: \alpha=.77)$.

Self-System Measures: Perceived Control. The Student Perceptions of Control Questionnaire (SPOCQ: Skinner, et al., 1988a; 1990) was used to measure 1) students' perceived control beliefs regarding the extent to which they believed they could control their experience of achieving academic successes and avoiding failures (generalized control beliefs), 2) the extent to which they believed five strategies (effort, ability, powerful others, luck, and unknown strategies) would achieve those outcomes (strategy beliefs), and 3) their perceptions of their capacity to access the known strategies (effort, ability, powerful others, and luck) to achieve those outcomes (capacity beliefs). Control beliefs were measured by six statements, strategy beliefs by four statements for each of 4 strategies and five statements for one additional strategy, and capacity beliefs by six statements for each of 4 strategies.

Examples of statements from this questionnaire measuring generalized control beliefs include "I can do well in school if I want to," and "I can't get good grades, no matter what I do" (reverse coded). To measure strategy beliefs, statements on the questionnaire include, "If I want to do well on my schoolwork, I just need to try hard," and "If I'm not smart, I won't get good grades" (reverse coded). Questionnaire items measuring capacity beliefs include "I would say I'm pretty smart in school," and "When I'm in class, I can't seem to work very hard" (reverse coded). Higher scores indicate a higher perception of general control and endorsement of a particular 
strategy or capacity. In use with grade school students, reliabilities for these scales have been adequate (control beliefs: $\alpha=.66$, averaged across measurement points in grades 3-7, [Skinner, et al.,1998]; $\alpha=.63$ in grades 3-6, [Furrer \& Skinner, 2003]; strategy beliefs: alphas ranged from .65 to .73 averaged across times of measurement in grades 3-6, [Skinner, et al., 1998]; capacity beliefs: alphas ranged from .64 to .75 averaged across times of measurement in grades 3-7, [Skinner, et al., 1998]).

Engagement. Students reported their level of behavioral and affective engagement and disaffection with classroom activities by responding to a 25 -item scale comprising two subscales. Ten items assess behavioral components, such as effort, persistence, and attention, and include items such as "I participate when we discuss new material," and "When I am in class, I just act like I'm working” (reverse coded). Fifteen items assess emotional involvement, with items such as "When we start something new in school, I feel interested," and "When I'm working on my classwork, I feel mad" (reverse coded). By averaging the two subscales together, a composite score for level of engagement is derived, with higher scores indicating more intensive involvement, and lower scores indicating greater disaffection.

Reliability for these scales in other studies has been consistently high $(\alpha=.79$, emotion, $\alpha=.81$, behavior, 3rd-5th graders, [Skinner \&Belmont, 1993]; $\alpha=.86$ emotion, $\alpha=.75$ behavior, 3rd-6th graders, [Furrer \& Skinner, 2003]; $\alpha=.76$ emotional engagement, $\alpha=.83$ emotional disaffection, $\alpha=.61$ behavioral engagement, $\alpha=.71$ behavioral disaffection, $3^{\text {rd }}-6^{\text {th }}$ graders, fall; $\alpha=.82$ emotional engagement, $\alpha=.85$ 
emotional disaffection, $\alpha=.72$ behavioral engagement, $\alpha=.78$ behavioral disaffection, $3^{\text {rd }}-6^{\text {th }}$ graders, spring, [Skinner, et al., 2009]; $\alpha=.88$ engagement versus disaffection [composite score], $3^{\text {rd }}-6^{\text {th }}$ graders, fall; $\alpha=.92$ engagement versus disaffection [composite score], $3^{\text {rd }}-6^{\text {th }}$ graders, spring, [Skinner, et al., 2009]).

Academic Achievement

Achievement scores for math and verbal skills (reading and/ or spelling) were obtained from student files. Multiple scores for each student in year 2 were available for a subset of the students who participated in the study. Students' scores were given in these subjects by their teacher in the form of a letter grade. In order to use these data for analysis, the letter grades were converted to numbers (ranging from 1 for " $F$ " or "U-," to 12 for "A" or "V") and then averaged across the year to represent level of academic achievement for each student for whom grades were available.

\section{Teacher Motivational Support}

Teacher report of provision of structure. While students were filling out their questionnaires, many teachers chose to respond to their own questionnaires regarding their interactions with their students. Teachers answered these items for each of the students they claimed to know best.

Amount of structure provided is measured by an 8-item scale tapping contingency, expectancy, instrumental help and support, and adjustment of teaching strategies. Examples of items include "I consistently apply consequences if this student doesn't meet the expectations," and "I change the rules about schoolwork for 
this student" (reverse coded). Reliabilities for this scale have been reported as adequate in previous research ( $\alpha=.70$, grades 3-5, [Skinner \& Belmont, 1993]).

Teacher report of involvement. The teacher involvement scale includes 16 items that tap a teacher's affection, attunement, dedication of resources, and dependability. Teachers responded to 16 items such as, "I enjoy the time I spend with this student," and "I don't understand this student very well" (reverse coded). Reliabilities for these items were reported as satisfactory in previous research $(\alpha=.83$, grades 3-5, [Skinner \& Belmont, 1993]).

Teacher report of provision of autonomy support. Teachers reported on their provision of autonomy support by answering 14 items that tap teacher coercive behavior (controlling behavior), respect, choice, and relevance. Items include statements such as "I let this student make a lot of his/ her own decisions regarding schoolwork," and "My general approach with this student is to give him/ her as few choices as possible" (reverse coded). Reliabilities for these items were reported as satisfactory in previous research $(\alpha=.90$, grades 3-5, [Skinner $\&$ Belmont, 1993]).

These scales are averaged together for use in the present study. Reliabilities for the composite scale have been reported as satisfactory in previous research $(\alpha=$ .95 , fall, $\alpha=.95$, spring, grades 3-7, [Skinner et al., 2008]). 


\section{CHAPTER 5: RESULTS}

\section{Descriptive Information}

Scale properties and descriptive statistics. All analyses were conducted using SPSS 21. Tables 8-10 (pp. 224-229) present the internal consistency reliabilities (Cronbach's alphas), means, standard deviations, and range statistics for all studentand teacher- report scales for the full sample and for each grade individually.

For the full sample, 15 of the 23 sub-scales showed adequate $(\geq .70)$ internal consistency reliabilities at the fall measurement point. The remaining eight were at or above $\alpha=.62$, with five scales improving to $\alpha \geq .70$ by spring. Student-report of Generalized Control Beliefs, and Strategy Beliefs for Effort and Ability displayed marginal improvement in spring, but were still below the acceptance threshold (.69, .64 , and .67 , respectively).

When disaggregated by grade, the scales for $4^{\text {th }}$ grade students clearly had weaker reliabilities at both time points than for $6^{\text {th }}$ grade students. Eleven of the 23 subscales showed weak reliabilities in the fall for $4^{\text {th }}$ grade students, with seven of those scales improving beyond the acceptance threshold by spring. The remaining four scales, Generalized Control Beliefs, Strategy Beliefs for Effort and Unknown strategies, and Capacity Beliefs for Effort, were all low in both fall and spring, with Capacity Beliefs for Effort in the fall showing the weakest reliability of any measure at $\alpha=.58$; while alphas for these four constructs did not improve enough to reach the .70 threshold, they did show a marginal increase by spring. Capacity Beliefs for Effort 
did show a relatively sizable increase from .58 to .66 , and Strategy Beliefs for Unknown strategies jumped from .60 to .69 , the largest increase of all four of these scales for $4^{\text {th }}$ graders. Two additional scales dropped below the acceptance threshold from fall to spring; Strategy Beliefs for Ability (.70 to .64) and Powerful Others (.70 to .69).

In contrast, the scales for the $6^{\text {th }}$ grade students showed more consistent reliabilities at each time point, with only 6 of the 23 scales showing low reliabilities in the fall, of which all but one improved above the acceptance threshold by spring. Capacity Beliefs for Luck was low in the fall $(\alpha=.66)$ and actually dropped a point by spring (.65). Additionally, the scale for Escape, which was quite strong in the fall $(\alpha=$ .79) dropped substantially by spring to a level below the threshold (.68). The attenuating effect of these weaker scale reliabilities should be kept in mind when conducting and interpreting the study analyses.

An examination of the minimum and maximum scores indicated that some students did report values equal to the anchors at either end of the response scale, with more scales having responses at the maximum value of the scale (4.0, indicating stronger endorsement of the concept) than at the minimum (1.0, indicating weaker endorsement) at both time points. This suggests there may be some positive bias in the responses due to ceiling effects. The standard deviations for all scales were moderate in size, ranging from .34 to .73 in the overall sample, .34 to .79 for the $4^{\text {th }}$ grade students, and .35 to .70 for the $6^{\text {th }}$ grade students. For most scales, the anchors 
of the scale fell within \pm 2 standard deviations of the mean. These observations suggest there is adequate variability in scale scores that should allow for detection of smaller effects.

Statistics for skewness and kurtosis were examined to determine the extent of positive or negative bias for each scale. The skewness statistic exceeded the acceptable level of 1.0 for only three variables for the $4^{\text {th }}$ grade students (fall Generalized Control in the fall, Powerful Others at both time points, and Confusion coping at both time points). Histograms for each of these variables were examined, and the distributions, while decidedly skewed, still followed a relatively symmetrical shape. Because predictor variables will be standardized for subsequent analyses, no further transformations for these variables were deemed necessary. All variables fell below the acceptable 2.5 cutoff in kurtosis analyses.

A restricted range of responses was noted for both grade groups for Total Strategy Beliefs and Total Teacher Support. The range of scores was more restricted for $4^{\text {th }}$ graders for Total Teacher Support, with a lowest minimum score of 2.18 and highest maximum of 3.90 , compared to $6^{\text {th }}$ graders with a lowest minimum of 1.82 and highest maximum of 3.96. Restrictions of range were more comparable between the grades for Total Strategy Beliefs, with lowest minimum scores of 1.09 for $4^{\text {th }}$ graders and 1.12 for $6^{\text {th }}$ graders, and highest maximum scores of 2.96 and 3.04 for $4^{\text {th }}$ and $6^{\text {th }}$ graders respectively. Restriction of range in responses was noted to a lesser degree for both grade groups in the Total Engagement construct as well. Subsequent analyses 
involving these constructs may have less power to detect group differences of smaller sizes due to decreased variability in these constructs.

Interpretation of means and mean level differences. When considered together as a group, students reported moderately high levels on average of Problem-Solving and Information-Seeking, and were less likely to report using Confusion or Escape as a means of coping. Confusion was endorsed more strongly than Escape, but not as strongly as either mastery-oriented way of coping. Students also perceived themselves to be behaviorally and emotionally engaged with classroom activities, and felt a strong sense of control over their academic outcomes. With respect to their sense of control, students reported a moderately high overall sense of control, and a moderately high capacity for accessing all four causes of attaining desired outcomes and avoiding negative ones, with Effort endorsed as the most effective strategy, and Powerful Others as the least effective. Students did not demonstrate much differentiation between the constructs with respect to internal versus external causes. Teachers perceived themselves to be moderately supportive of students overall, particularly in their provision of structure.

Table 20 (pp. 240-241) reports the results of t-tests conducted to determine whether mean level differences between groups on each construct are significant at each time point.

Control. Students in both grades felt a strong sense of generalized control at the beginning of the year, with no significant difference between the grades (Table 20, 
pp. 240-241). While $4^{\text {th }}$ grade students maintained this perception across the year, $6^{\text {th }}$ grade students reported significant decreases in a general sense of control by spring, which also constituted a significant difference in the amount of change in the construct from fall to spring between the grades, resulting in a corresponding significant between-groups difference on the spring measure of this construct (Tables 9-10, pp. 226-229).

Capacity Beliefs. While both groups of students reported a moderately high capacity for accessing all four success strategies in the fall (Tables 9-10, pp. 226-229), $4^{\text {th }}$ grade student ratings of capacity were significantly higher than those of $6^{\text {th }}$ grade students on all four strategies at both time points (Table 20, pp. 240-241). Both groups reported significant decreases in the spring in their capacity to access Effort and Luck. Fourth grade students maintained a sense of control across the year with respect to their capacity to access Ability and Powerful Others, with only slight decreases in endorsement of their capacity for these strategies, but $6^{\text {th }}$ grade students reported significant declines for these strategies as well (Tables 9-10, pp. 226-229). The direction and significance of mean level differences between the grades on all capacity beliefs noted in the fall remained unchanged in the spring; namely, $4^{\text {th }}$ grade students reported significantly higher levels of confidence than $6^{\text {th }}$ grade students in their capacity to access all four success strategies across the entire year (Table 20, pp. 240-241). 
Table 22 (p. 247) reports the results of t-tests conducted to determine whether the amount of change from fall to spring in each construct is significantly different between the grades. The decrease in perceptions across the year of capacity to access strategies for success noted for $6^{\text {th }}$ grade students, while larger than the changes noted for $4^{\text {th }}$ graders, were not significantly different from them.

Strategy Beliefs. Effort and Ability were endorsed by both groups as effective strategies for success, which was maintained by both across the year (Tables 9-10, pp. 226-229). Between-group differences in the means were noted for Strategy Beliefs for Effort, but not for Ability, with $6^{\text {th }}$ grade students showing significantly stronger endorsement of Effort as a success strategy than $4^{\text {th }}$ grade students in the fall (Table 20, pp. 240-241). These positions reversed in the spring, with $4^{\text {th }}$ grade students having significantly stronger endorsement of Effort as a success strategy than $6^{\text {th }}$ graders. Despite this change in mean-level endorsement of Effort as a strategy for success, significant differences in the amount of change noted from fall to spring for each group were not found (Table 22, p. 247). No significant differences in changes for Strategy Beliefs for Ability were found.

Endorsement of Strategy Beliefs for Powerful Others, Luck, and Unknown strategies was weaker than that of the other two strategies for both groups (Tables 910 , pp. 226-229), with $6^{\text {th }}$ graders showing significantly stronger endorsement than $4^{\text {th }}$ graders of Powerful Others at both time points (Table 20, pp. 240-241). Significant mean level differences were also found for Luck in the fall, and Unknown strategies in 
the spring, with $4^{\text {th }}$ graders showing stronger endorsement than $6^{\text {th }}$ graders of Luck in the fall, and $6^{\text {th }}$ graders showing stronger endorsement than $4^{\text {th }}$ graders of Unknown strategies in the spring. Changes from fall to spring were significant for students in both grades for Luck and Unknown, and for $6^{\text {th }}$ graders only for Powerful Others (Tables 9-10, pp. 226-229). The amount of change from fall to spring differed significantly between the grades on all three constructs, with larger mean changes for $6^{\text {th }}$ grade students on Powerful Others and Luck, and larger changes for $4^{\text {th }}$ graders on Unknown Strategies (Table 22, p. 247).

Note that while the difference between the grades in the amount of change demonstrated is significant, this does not mean that the resulting overall means in the spring are also significantly different (Table 20, pp. 240-241). For example, a significant difference between groups was found in the fall for Luck, with $4^{\text {th }}$ graders showing stronger endorsement than $6^{\text {th }}$ graders; $6^{\text {th }}$ graders made a larger change from the fall in their spring endorsement than did the $4^{\text {th }}$ graders, bringing the mean ratings for each group in the spring closer together, so that there was no longer a difference between them. Likewise, where there was not a significant difference between groups in the fall for Unknown strategies, $4^{\text {th }}$ graders made a larger change in their ratings in the spring than did $6^{\text {th }}$ graders, resulting in a significant difference between the groups in the spring, with $6^{\text {th }}$ graders now at a higher overall mean level for Unknown strategies than $4^{\text {th }}$ graders. 
Coping. As shown in Tables 9-10 (pp. 226-229), both $4^{\text {th }}$ and $6^{\text {th }}$ grade students showed moderately high endorsement of both mastery-oriented ways of coping, with stronger endorsement by the $4^{\text {th }}$ grade students. Endorsement was stronger for Confusion coping than for Escape coping for both grades, with $6^{\text {th }}$ graders endorsing more strongly than $4^{\text {th }}$ graders on both ways of coping. No significant changes in endorsement from fall to spring in any way of coping was noted for $4^{\text {th }}$ grade students, but changes for $6^{\text {th }}$ grade students were significant for all ways of coping except Confusion. Endorsement of mastery coping declined significantly for $6^{\text {th }}$ grade students, but held steady for $4^{\text {th }}$ grade students, with only slight, nonsignificant declines. Endorsement of Confusion coping increased for $6^{\text {th }}$ grade students and decreased for $4^{\text {th }}$ grade students but was not a significant change. Endorsement of Escape coping increased for both grades, and significantly so for $6^{\text {th }}$ graders. In spite of the divergence in endorsement, Confusion coping was still more strongly endorsed than Escape coping at both time points by both grades.

Mean-level differences between grades were significant for InformationSeeking and Escape at both time points, and for Problem-Solving only on the spring measure; no significant difference was found for Confusion coping at either time point (Table 20, pp. 240-241). Students in both grades showed a decrease in their endorsement of Problem-Solving in the spring, and an increase in their endorsement of Escape. The $6^{\text {th }}$ grade students showed a significantly larger change than the $4^{\text {th }}$ grade 
students for both Problem-Solving and Escape (Table 22, p. 247). Grade differences for changes in Information-Seeking and Confusion were not significant.

Engagement. Both $4^{\text {th }}$ and $6^{\text {th }}$ grade students reported moderately high levels of both types of engagement at both time points (Tables 9-10, pp. 226-229), with $4^{\text {th }}$ grade students reporting significantly higher behavioral and emotional engagement than $6^{\text {th }}$ grade students in both the fall and spring (Table 20, pp. 240-241). Both groups of students showed significant decreases in overall engagement from fall to spring, but while $6^{\text {th }}$ grade students showed significant decreases in both types of engagement, $4^{\text {th }}$ grade students evidenced significant decreases in behavioral engagement only; no significant change occurred for $4^{\text {th }}$ grade students in emotional engagement (Tables 9-10, pp. 226-229). The changes in both behavioral and emotional engagement from fall to spring were significantly larger for $6^{\text {th }}$ grade students than for $4^{\text {th }}$ grade students (Table 22, p. 247).

Teacher Support. Tables 9-10 (pp. 226-229) show that teachers reported moderately high levels of support for students in both grade groups consistently across time, with provision of Structure the strongest means of support provided in both grades at both time points. Teachers in the $4^{\text {th }}$ grade did not differ significantly from fall to spring in their perception of the level of overall support they were giving their students, but $6^{\text {th }}$ grade teachers did perceive themselves to be more supportive in the spring than in the fall. From fall to spring, $4^{\text {th }}$ grade teachers reported a significant decrease in provision of Structure, with a corresponding significant increase in 
Autonomy Support, and no change in provision of Involvement, while $6^{\text {th }}$ grade teachers significantly increased their provision of both Structure and Involvement, with no change in Autonomy Support.

Compared to $6^{\text {th }}$ grade teachers, $4^{\text {th }}$ grade teachers rated their level of overall support significantly higher in the fall than $\operatorname{did} 6^{\text {th }}$ grade teachers, and specifically higher in their provision of Structure in the fall, and Autonomy Support in both the fall and spring (Table 20, pp. 240-241). No significant differences in perceptions of provision of Involvement were found between the two groups of teachers at either time point. By spring, significant differences between groups on perceptions of all forms of support except Autonomy Support were no longer noted.

The difference between the two groups of teachers in the magnitude of fall to spring changes was significant for overall teacher support, with a larger change from fall to spring for the $6^{\text {th }}$ grade teachers than the $4^{\text {th }}$ grade teachers. For individual types of support, only the provision of Structure showed a significant difference in the amount of fall to spring change between the grades, with the increase made by the $6^{\text {th }}$ grade teachers significantly larger than the decrease made by the $4^{\text {th }}$ grade teachers (Table 22, p. 247). In spite of the equalizing direction of these changes, the $4^{\text {th }}$ grade teachers continued to report a higher mean on both overall teacher support and provision of Structure than the $6^{\text {th }}$ grade teachers (Tables 9-10, pp. 226-229), although the mean-level differences between them were no longer significant for either measure (Table 20, pp. 240-241). 
Stability over time. Tables 8-10 (pp. 224-229) also provide the correlations for each scale from fall to spring for the full sample and for each grade individually. All scales for each of the three groups displayed a high level of stability over time, ranging from a low of $r=.45$ noted in each of the groups, to a high of $r=.79$ for the full sample, $r=.80$ for the $6^{\text {th }}$ graders, and $r=.85$ for the $4^{\text {th }}$ graders, (average $r=.63$ $-.65)$. Given the general stability of the constructs, it may be difficult to detect significant differences that are small due to decreased variability over time. Stability was also examined with respect to age differences by testing the difference in the fallto-spring correlations between grades. This was accomplished through regressions using the spring constructs as the dependent variables, and the fall construct, grade, and the construct * grade interaction term as the independent variables. Significant interaction terms indicate a difference in the correlations by grade. Table 21 (pp. 242246) provides the regression statistics for each construct.

Significant interaction terms were noted for Capacity Beliefs for Effort, and teacher provision of both Structure and Autonomy Support. For each of these constructs, the regression was conducted again for each grade individually and regression statistics compared to determine the direction of the difference. The effect for Capacity Beliefs for Effort was stronger for $6^{\text {th }}$ graders compared to $4^{\text {th }}$ graders, with the fall construct accounting for $46 \%$ of the variance in the spring construct $\left[\mathrm{R}^{2}=\right.$ $.46, \mathrm{~F}(1,334)=$ range: $219.796-357.205, \mathrm{p}<.001]$, compared to only $21 \%$ for the $4^{\text {th }}$ grade students $\left[\mathrm{R}^{2}=.21, \mathrm{~F}(1,327)=\right.$ range: $\left.62.777-114.329, \mathrm{p}<.001\right]$. The 
effects for both of the teacher constructs were stronger for $4^{\text {th }}$ grade students compared to $6^{\text {th }}$ grade students. For teacher provision of Structure, the fall construct accounted for $63 \%$ of the variance in the spring construct $\left[\mathrm{R}^{2}=.63, \mathrm{~F}(1,334)=\right.$ range: 487.425 $-671.886, \mathrm{p}<.001]$, compared to only $33 \%$ for the $6^{\text {th }}$ grade students $\left[\mathrm{R}^{2}=.33, \mathrm{~F}(1\right.$, $327)=$ range: $144.073-181.729, \mathrm{p}<.001]$. For teacher provision of Autonomy Support, the fall construct accounted for $65 \%$ of the variance in the spring construct $\left[\mathrm{R}^{2}=.65, \mathrm{~F}(1,334)=\right.$ range: $\left.517.129-674.259, \mathrm{p}<.001\right]$, compared to $56 \%$ for the $6^{\text {th }}$ graders $\left[\mathrm{R}^{2}=.56, \mathrm{~F}(1,327)=\right.$ range: $\left.382.748-506.868, \mathrm{p}<.001\right]$. These findings suggest that adequate variability to detect small effect sizes in subsequent analyses is present in the data for Capacity Beliefs for Effort for the $6^{\text {th }}$ graders and teacher provision of Structure for the $4^{\text {th }}$ graders. The construct for teacher provision of Autonomy Support, however, demonstrates much less variability between the grades, in spite of the significant difference in the fall to spring correlations, suggesting a potential for increased difficulty in detecting significant effects in subsequent analyses.

Intra-construct correlations. Correlations between subscales within each construct were examined for the overall sample to ensure correlations are neither too high $(r>.90)$, nor too low $(r<.30)$. Tables 11-13 (pp. 230-232) show the correlations obtained for the subscales of the perceived control constructs for the full sample and by individual grade, of which significant correlations run from absolute values of .003 to .77. In general, the constructs for Capacity Beliefs and Strategy Beliefs are 
moderately and negatively correlated, with the exception of Strategy Beliefs for Effort, which is either not correlated with individual Capacity Beliefs, or where it is correlated, the correlation is exceptionally low. Across the full sample, low correlations are seen between Strategy Beliefs for Effort and Capacity Beliefs for Powerful Others in the fall, and Capacity Beliefs for Ability and Luck in the spring. Other components of Capacity Beliefs are not correlated with Strategy Beliefs for Effort at all.

By grade, the $4^{\text {th }}$ grade students show a weak correlation for Strategy Beliefs for Effort only with Capacity Beliefs for Luck in the spring. Sixth grade students do show weak correlations between Strategy Beliefs for Effort with all Capacity Beliefs in the fall, but no significant correlations are found in the spring. All other constructs in the Strategy Beliefs set are correlated with Capacity Beliefs constructs, at low to moderate levels for both grades at both time points. The correlations for Strategy Beliefs for Ability, while significant at both time points, are lower than the $r=.30$ threshold for both grade groups. This should be taken into account when interpreting subsequent analyses using the control aggregate constructs.

Examination of correlations between subscales for Engagement and Teacher Support showed that the correlations for Engagement were well within expected ranges $(r=.60-.72)$, with both grades showing similar patterns of positive relationships between the two components of Engagement. Teacher Support evidenced low correlations for provision of Structure and Involvement at both the fall 
and spring time points ( $r=.09$ and .21 , respectively for the full sample), which appears to be driven by the lack of any significant correlation between these two components for the $4^{\text {th }}$ grade students. It is important to note that even though these correlations are lower than what is considered optimal, they are, however, correlated at a moderate to high significance level $(p<.01-p<.001)$. Correlations between the other components were positive and significant for both grades, as expected.

Inter-construct correlations. Tables 17-19 (pp. 236-239) display correlations between constructs used in subsequent analyses for the full sample and each grade individually. These correlations were examined as an indicator of the hypothesized relationships tested in this study. Non-significant relationships suggest further investigation of questions pertaining to those relationships is not warranted. All interconstruct correlations demonstrated significant relationships, with the exception of overall Achievement with some ways of coping, and in differing patterns by grade. While the correlations for the full sample showed all ways of coping except Confusion coping in the spring significantly related to Achievement, the individual grade correlations revealed a different pattern.

In the fall, neither mastery way of coping showed a significant relationship with Achievement for the $4^{\text {th }}$ grade students. These relationships for the $6^{\text {th }}$ grade students were significant and positive, but weak. By spring, mastery coping for the $4^{\text {th }}$ grade students was significant and positively related to Achievement, with moderate strength. For $6^{\text {th }}$ grade students, however, a slight decline was seen in Problem- 
Solving from fall to spring, and Information-Seeking coping was no longer significantly related to Achievement. Helplessness coping was significantly related to Achievement for both grades in the fall, but the relationship for Escape coping was much stronger than for Confusion coping, and Confusion coping was weakly but positively related to Achievement for the $4^{\text {th }}$ grade students, and weakly but negatively related to Achievement for the $6^{\text {th }}$ grade students. In the spring, the strength of the relationship with Escape coping increased for $4^{\text {th }}$ graders, but stayed essentially the same for $6^{\text {th }}$ graders. Confusion coping was no longer significantly related to Achievement in the spring for $4^{\text {th }}$ grade students, but became weakly and positively related to Achievement for the $6^{\text {th }}$ grade students. These patterns follow expected trends for the younger children, but are contrary to expectations for the older children. Cognitive abilities are believed to increase with age and facilitate access to higher achievement. These findings suggest that the link between coping strategies and Achievement may be influenced by other processes that are not apparent in the direct relationship.

According to the causal steps approach to mediational analysis (Baron \& Kenny, 1987), this finding would suggest that in subsequent analyses the mediational models involving mastery ways of coping in the fall, Information-Seeking in the spring, and Confusion coping in the spring should not be tested due to the lack of the requisite significant direct effects relationships between these ways of coping and Achievement for both groups of students. Recent research in advanced methodologies 
for testing mediation hypotheses provides a contrary perspective (Hayes, 2009; 2013; 2015). In sum, Hayes $(2009 ; 2013 ; 2015)$ offers evidence that the indirect effect of a predictor variable can be carried through a mediating variable even when there is no direct relationship between the predictor and the dependent variable. Further discussion of this work follows in the next section that details the analysis strategies chosen to test the study hypotheses. Based on the examination of the inter-construct correlations described here and the evidence from Hayes $(2009 ; 2013 ; 2105)$ as subsequently presented, mediational models for all four ways of coping at both time points were tested, despite the lack of significant correlation between certain ways of coping and achievement for some students at some measurement points, in an effort to illuminate the possibility of intervening processes that may better explain the link between coping and Achievement.

Assessment of potential multicollinearity. Examination of all correlations by grade for evidence of multicollinearity revealed no correlation between the constructs greater than .78, suggesting multicollinearity would not be an issue in subsequent analyses. An exception to this observation is noted between Total Capacity Beliefs and CONMAX $(r=.94-.97)$. The high correlation between these constructs is not a concern, as they are not used together in any analysis, and in fact suggests that planned analyses using CONMAX could be conducted with Total Capacity Beliefs instead, and vice versa. Centering predictor variables is another strategy for protecting against the effects of multicollinearity. Due to the requirements of pooling the results of 
analyses from datasets obtained through multiple imputation to address issues of missing data, all predictor variables have been standardized rather than mean-centered. The effect with regards to multicollinearity is equivalent.

Details of the subsequent analyses are organized around the four primary research questions of this study, following an overview of the analysis strategies used for various hypotheses that includes a discussion of the rationale for the specific methodology chosen to test the hypothesized mediation models. 


\title{
Analyses Overview
}

\author{
Analysis Strategy
}

The primary study analyses can be categorized as one of three types:

1. Age differences in relationships between concurrent measures

2. Age differences in predicting change over time

3. Age differences in mediational relationships

Concurrent measures. As noted previously in the literature review, concurrent prediction is not true prediction, as it is based on concurrent correlations. Analyses to detect age differences in the relationships between concurrent measures are conducted through regressions that incorporate an interaction term. These analyses take the form depicted in Figure 12, with two predictor variables and their cross-product (interaction term). Cross-product variables are obtained by multiplying the main effects variables together to create a new variable that represents the interaction of the variables used to create it.

Concurrent measures analyses include constructs from the same time-point, either time 1 or time 2 , for both the IV and DV. A significant interaction term will indicate that the amount of variance accounted for in the DV by the IVs differs with age. Because this study examines only two grade groups, the interaction term is

computed using grade as a dummy-coded variable, with $4^{\text {th }}$ grade used as the reference group. 


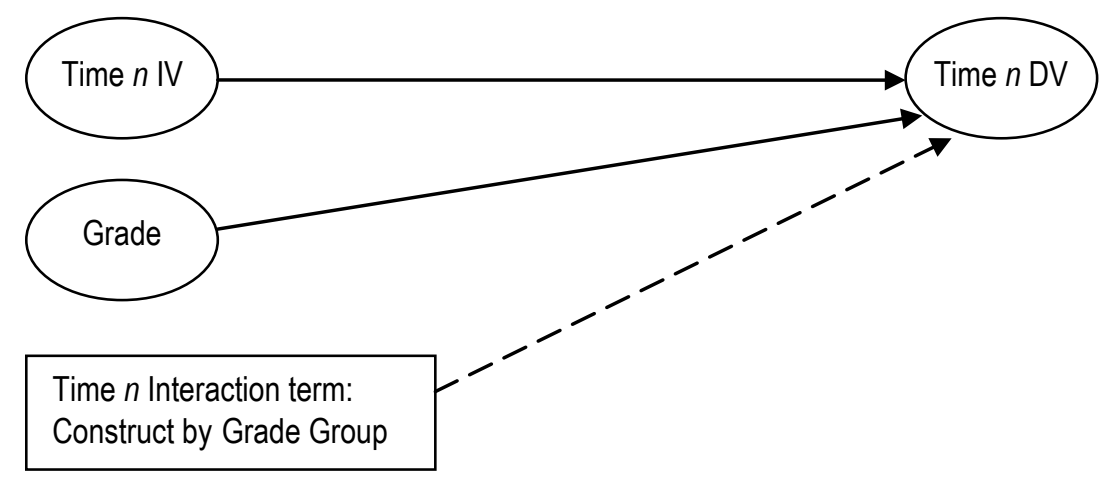

Figure 12: Model for analyses to test age differences in the relationship between concurrent measures.

Keeping in mind that all IVs have been standardized to facilitate the pooling of the results for each of the datasets obtained through the multiple imputation approach to handling missing data, and that the grade variable has been dummy coded, interpretation of the regression results are made as follows: the coefficient for the construct (IV) in the model (direct effect) is the amount of change in the mean of the DV when all predictors in the model are at zero (the constant) that occurs for every one standard deviation increase in the construct mean. Two important points should be highlighted here: because the $4^{\text {th }}$ grade group is the reference group, 1) the constant, which is the mean on the DV when all predictors in the model are zero, is the mean on the $D V$ for the $4^{\text {th }}$ grade group (the reference group), and 2) there is no adjustment to the IV coefficient for the $4^{\text {th }}$ grade group. The IV coefficient represents the increase in the $4^{\text {th }}$ grade mean on the DV (the constant) that occurs for every one standard deviation increase in the mean of the IV.

The coefficient for the grade variable represents the difference between the means of the two grade groups when all other predictors in the model are at zero, or in 
other words, in their intercepts. In practical terms, this coefficient is the amount of change from the constant (the mean of the DV for the reference group) when all other predictors in the model are zero for the $6^{\text {th }}$ grade group, which is the intercept for the $6^{\text {th }}$ graders. If the grade variable coefficient is significant, it means the two groups differ significantly on their respective intercepts. If the coefficient is negative, it means the $6^{\text {th }}$ grade group has a lower mean on the DV (because it would be added to the constant) or in graphical terms, a lower intercept. If the coefficient is positive, it means the $6^{\text {th }}$ grade group has a higher mean on the DV, or a higher intercept.

The coefficient for the interaction term is the difference in the slopes of the regression lines specified by the equation for each group. If the coefficient is significant, then the magnitude of change in the mean of the DV that occurs as the IV mean increases is significantly different for the $6^{\text {th }}$ grade students compared to the $4^{\text {th }}$ grade students. In practical terms, the interaction coefficient is an adjustment to the coefficient of the IV; the size and direction of both coefficients determines the magnitude of the difference in the slopes. The interaction coefficient is added to the IV coefficient for the $6^{\text {th }}$ grade group to derive the amount of change from the intercept for the $6^{\text {th }}$ grade group that occurs for every one standard deviation change in the IV.

Change over time. Analyses to detect age differences in the prediction of change in a measure from time 1 to time 2 are accomplished through regressions of the form illustrated by Figure 13. The DV is the measure of that construct at time 2, and the time 1 measure of the DV is added to the equation as an IV ahead of the main 
effects variables, in order to control for the contribution of previous levels of the DV. This leaves only the variance unaccounted for by previous levels of the DV to be accounted for by the main effects variables. The cross-product of the main effects variables is included as the interaction term.

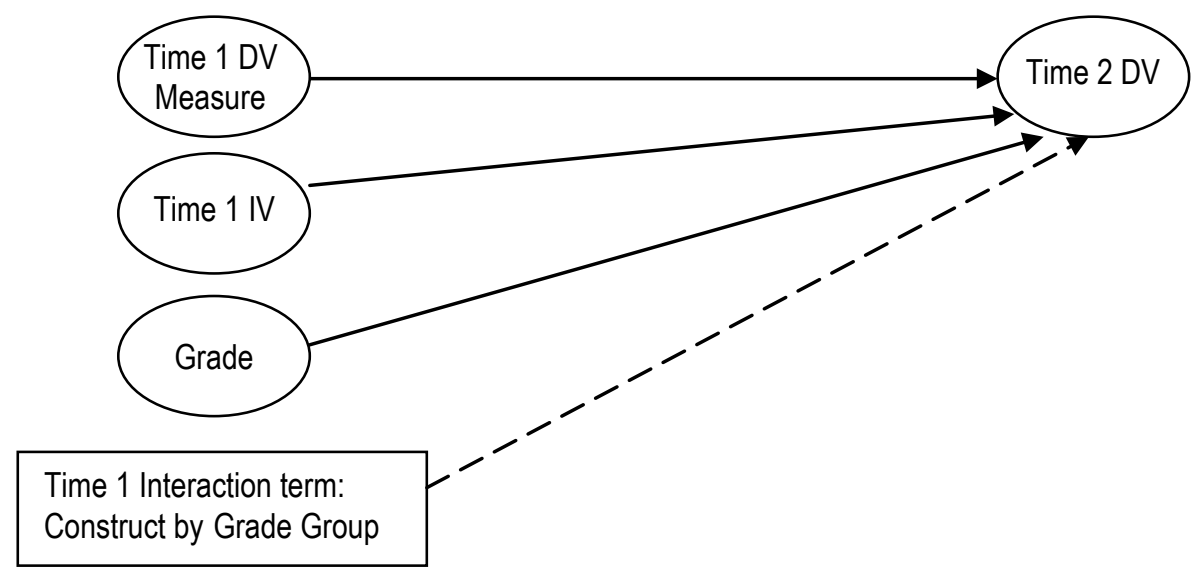

Figure 13: Model for analyses to test age differences in the prediction of change over time.

A significant interaction term indicates that the amount of variance accounted for in the DV by the IVs, after controlling for the contribution of the time 1 measure of the DV, differs with age.

Regression coefficients are interpreted as indicated for the previous model, with this addition for the first step of the equation: the construct coefficient in the first step of the model is the change in the DV mean for all students when the construct and all other constructs in the model are at zero. The $\mathrm{R}^{2}$ statistic represents the amount of variance in the DV accounted for by initial levels of the DV. The $\Delta \mathrm{R}^{2}$ statistic represents the amount of additional variance in the DV the second step of the model 
accounts for, over and above the variance accounted for in the first step. Each statistic is accompanied by a significance test based on the F-distribution.

Mediation analyses. Many experts in the field of methodology have offered a definition of mediation (Baron \& Kenny, 1986; Edwards \& Lambert, 2007; Hayes, 2009; James \& Brett, 1984; MacKinnon, Lockwood, Hoffman, West, \& Sheets, 2002; Preacher, Rucker, \& Hayes, 2007; Shrout \& Bolger, 2002). While the definitions are all slightly different and may emphasize one conceptual notion or another, there appears to be consensus that a mediated relationship, in its simplest form, involves a predictor, denoted as $X$, and a dependent variable, $Y$, wherein the influence of $X$ on $Y$ is partially or fully carried through a third intervening variable called a mediator, denoted as $M$.

History and critique. For many years the most commonly accepted method for testing mediational relationships has been the four-step process outlined by Baron and Kenny (1986), known as the causal steps approach. This approach specifies four specific conditions, outlined in Figure 14, which must be met in order to make a valid claim of partial or full mediation. Over the past ten years, this approach has been criticized on a number of points, and the Sobel test mentioned in step 4 shown to be inadequate on the basis that it is an inference of mediation, not a direct test of the coefficients that produce the effect of interest (Edwards \& Lambert, 2007; Hayes, 2009). 
Edwards and Lambert (2007) highlight several limitations of the approach, including the requirement of a significant relationship between $X$ and $Y$. The problem with this requirement can be understood mathematically. Since the effect of $X$ on $Y$ and the indirect effect of $X$ on $Y$ as carried through $M$ sum to the total effect, it is possible that a significant indirect effect could be masked by a direct effect of similar size, but opposite sign. In the first step of the causal steps approach, the coefficient in the regression that tests the direct effect of $X$ on $Y$ is the same as the coefficient for the overall effect, because the equation is not testing the mediated path and that coefficient is not separated out; but if the direct effect comprises significant contrary effects, it may not reach significance, and the mediated effects would not be discovered. Given the logic of the mathematics, an effect that is not present directly may still be mediated through an intervening variable.

Edwards and Lambert (2007), as well as others (Hayes, 2009; MacKinnon et al., 2002; Preacher et al., 2007; Shrout \& Bolger, 2002) offer support for additional criticism through mathematical demonstration of how the causal steps approach does not directly test the mediated effect of $X$ on $Y$ as represented by the mathematical quantification of the full indirect pathway through the mediating variable, which is obtained as the product of the coefficients of the component paths in the model.

These issues and the general lack of knowledge on the part of field researchers as to the appropriate treatment of them are particularly apparent when the question of interest involves testing whether an indirect effect varies significantly by a fourth 
Step 1: Establish a significant relationship between the IV and the DV (there is an effect subject to mediation).

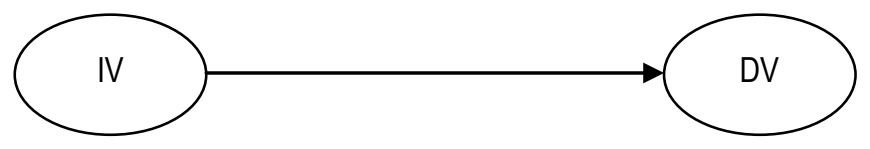

Step 2: Establish a significant relationship between the IV and the mediator.

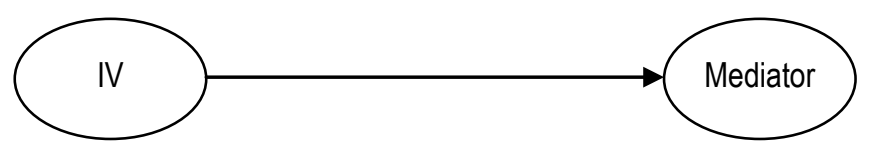

Step 3: Establish a significant relationship between the mediator and the DV, controlling for the $I V$.

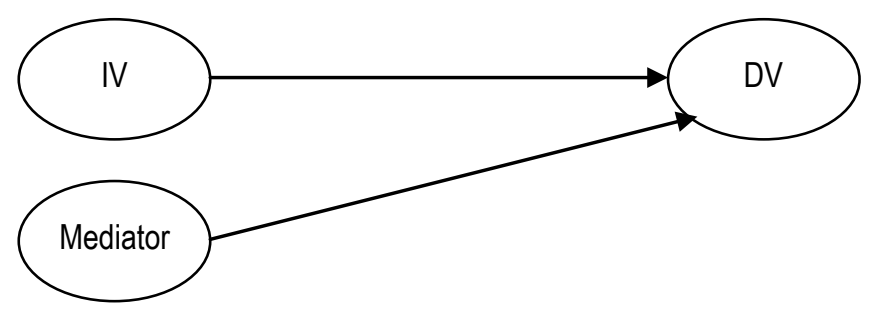

Step 4: To establish complete mediation, the relationship between the IV and the DV should no longer be significant when the mediator is included in the model. Partial mediation is indicated if the pathway between the IV and the DV remains significant but is substantially reduced. Baron \& Kenny (1986) refer to this as the Sobel test.

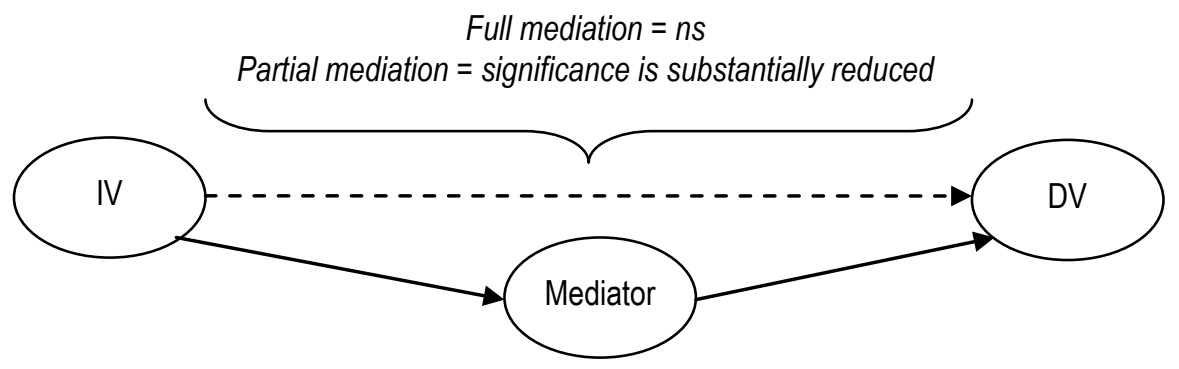

Figure 14: Four steps to establish a mediational relationship (Baron \& Kenny, 1986). 
variable called a moderator, denoted by $W$. Edwards and Lambert (2007) describe the variety of methods used to test models that combine moderation and mediation in studies they reviewed as part of their critique, and provide evidence of the inherent shortcomings of those methods.

Of particular concern, they noted that most studies reviewed reported only a portion of the coefficients necessary for a complete and substantive interpretation of the model under investigation. They follow this review and critique of current practice with detailed information and illustration of a framework that builds on the work of pioneers in the field (Baron \& Kenny, 1986; James \& Brett, 1984) by incorporating concepts from path analysis and structural equation modeling. This framework then leads to a methodology for testing and reporting on simple and complex models that integrate moderation and mediation, which also avoids the pitfalls noted to date.

Conditional effects models. Earlier discussions among methodologists focused on the terminology to be used in describing such models as well as the mathematical formulations (Muller, Judd, \& Yzerbyt, 2005). The distinctions between "moderated mediation" and "mediated moderation" are not insignificant, and have important mathematical implications for how the equations are specified. The distinctions are, however, conceptually confusing. To ameliorate this confusion and move the discussion forward, others in the field advocated for a more generalized description of models of both kinds as "conditional effects" models, and the methods used to 
understand them as "conditional process analysis" (Hayes, 2009; Preacher, et al., 2007). When approached from this conceptualization, emphasis is placed on correct specification of the equations that comprise the model, ensuring the mathematical accuracy of the analysis. Upon examination of those equations, it becomes clear that when one is talking about moderated mediation, the mathematical implication is that if the indirect effect of $X$ on $Y$ through $M$ can be shown to be a function of (conditional on) another variable, that function can be subjected to a direct test of the null hypothesis to determine whether the indirect effect is moderated by that variable. In Hayes (2013) methodology, it is the coefficient of the moderator variable in this function that is tested to determine whether it differs significantly from zero. Hayes work $(2013 ; 2015)$ focuses on explicating this methodology by extending the work of Edwards \& Lambert (2007) and creating tools for field researchers to use that make it easy to conduct a direct test of this relationship rather than relying on the methodology of the causal steps approach that infers moderated mediation from tests of the component pathways.

A brief look at the derivation of the relevant equations that produce the coefficients helps to highlight the difference between these two methods, and the practical value of the notion that the relationship of interest, that is, the relationship between the indirect effect of $X$ on $Y$ through $M$ and the moderator, $W$, can be specified as a mathematical function. 
Figure 15 depicts the path model for a simple mediation model. Assuming $Y$ and $M$ are continuous and the relationships in the model are linear, the model is specified by two regression equations:

$$
\begin{gathered}
M=i_{M}+a X+e_{M} \\
Y=i_{Y}+c^{\prime} X+b M+e_{Y}
\end{gathered}
$$

where $i_{M}$ and $i_{Y}$ are the regression intercepts, $a, b$, and $c^{\prime}$ are the estimated regression coefficient terms, and $e_{M}$ and $e_{Y}$ are the errors in estimation. Equation 1 specifies the first stage path $(X \rightarrow M)$, and Equation 2 specifies the direct path $(\mathrm{X} \rightarrow \mathrm{Y})$ and the second stage path $(\mathrm{M} \rightarrow \mathrm{Y})$. The indirect effect of $X$ on $Y$ through the mediating variable $M$ is quantified by the product of the coefficient terms $a$ and $b$.

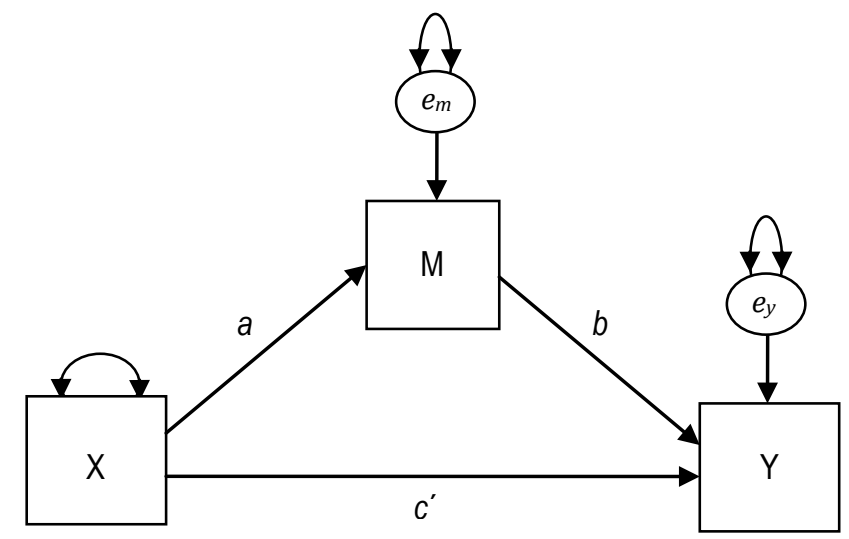

Figure 15: Path model for simple mediation.

Through substitution and combination of terms the equation then for the total effect of $X$ on $Y$, accounting for the mediating effect of $M$, is given as:

$$
Y=i_{Y}+c^{\prime} X+a b X+e_{Y}
$$


The estimated product coefficient term, $a b X$, is the resulting quantification of the indirect effect of $X$ on $Y$ through $M$, and is interpreted as the amount by which two variables that differ by one unit on $X$ can be expected to differ on $Y$ through the effect of $X$ on variable $M$, which in turn exerts influence on $Y$. The estimated regression coefficient $c^{\prime} X$ is the quantification of the direct effect of $X$ on $Y$, and is interpreted as the part of $X$ which influences $Y$, independent of the pathway through $M$. The sum of the direct and indirect effects represents the total effect for the model.

The equation for a simple moderator model is more straightforward, as it does not need to be broken into component parts. Figure 16 depicts the path model for a simple moderator model.

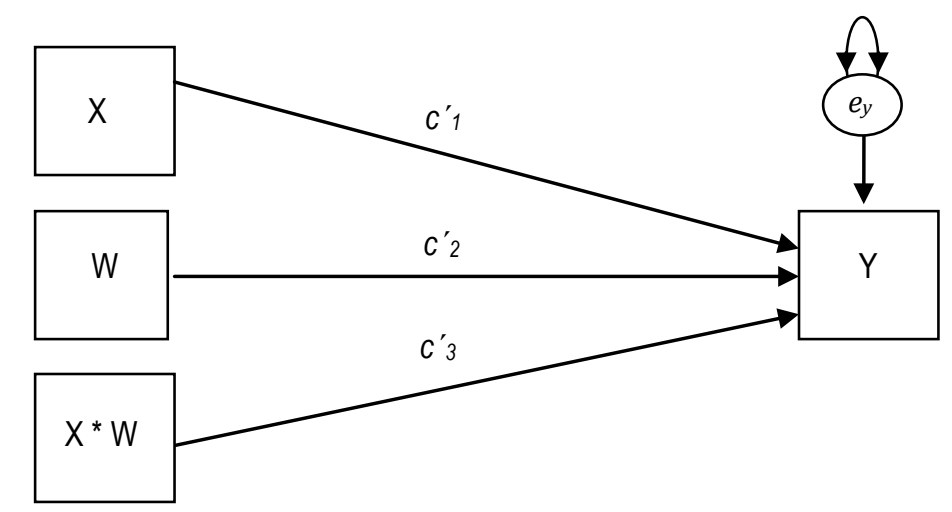

Figure 16: Path model for a simple moderator model.

The equation for testing the contingency of the effect of $X$ on $Y$ due to variations in $W$ is given as:

$$
Y=i_{Y}+c^{\prime}{ }_{1} X+c^{\prime}{ }_{2} W+c^{\prime}{ }_{3} X W+e_{Y}
$$


where $i_{Y}$ is the intercept, $c{ }_{1} X, c^{\prime}{ }_{2} W$ and $c^{\prime}{ }_{3} X W$ are the estimated regression coefficients, and $e_{Y}$ is the error in estimation. In this equation, the product coefficient $c^{\prime}{ }_{3} X W$ represents the magnitude of the effect of $X$ on $Y$ at varying levels of $W$. Moderation is inferred when this coefficient is deemed statistically different than zero.

Figure 17 depicts the path model that results when the question of interest involves testing the contingent effect of a moderator, $W$, on both the direct and indirect paths of influence of $X$ on $Y$ through a mediator $M$. This model is specified by two equations derived by incorporating the coefficient terms for the moderator and its interaction with $X$ from Equations 3 and 4 into the equation for $M$ (Equation 1) and into the equation for $Y$ that includes the effect of the mediator $M$ on $Y$ (Equation 2):

$$
\begin{gathered}
M=i_{M}+a_{1} X+a_{2} W+a_{3} X W+e_{M} \\
Y=i_{Y}+c^{\prime}{ }_{1} X+c^{\prime}{ }_{2} W+c^{\prime}{ }_{3} X W+b_{1} M+b_{2} M W+e_{Y}
\end{gathered}
$$

From here, if the two equations were further integrated to yield one final equation for the total effects of the model, one would be faced with the question of possible non-linear relationships that result when the moderator is a continuous variable. Edwards and Lambert (2007) outline a methodology for testing hypotheses when the moderator is continuous; however, the moderator in the present study is a dichotomous variable indicating grade in school as a proxy for age. Hayes' methodology (2013) is applicable only to linear models where the moderator is an ordinal variable and, as such, is well suited to the needs of the present study. 


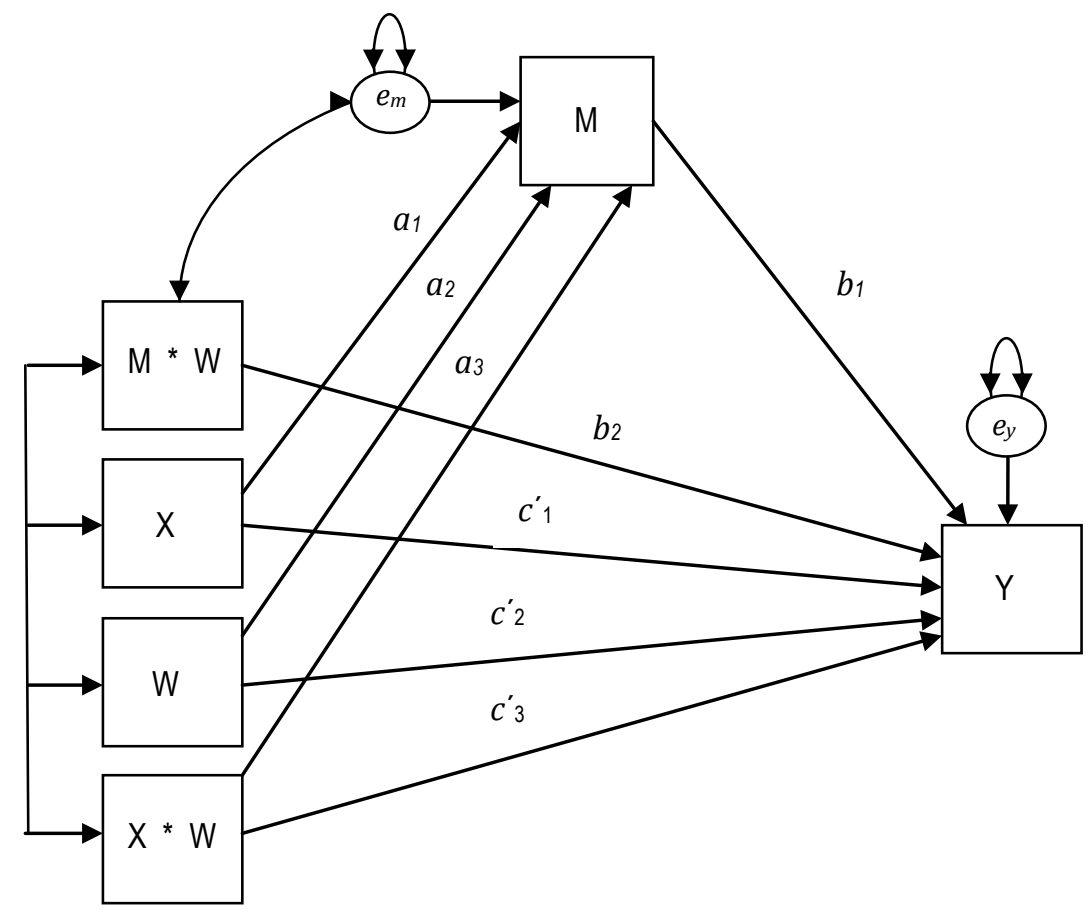

Figure 17: Path model for multi-group interaction model depicting interaction terms for both the IV and the moderator; adapted from Preacher, Rucker, \& Hayes, 2007.

For this reason, this discussion will continue with Equations 5 and 6 kept separate, as further integration would yield non-linear terms that, because the moderator is dichotomous and dummy-coded in the present analyses, are not relevant (i.e., the terms would reduce to zero or one, given the dummy coding used). Hayes $(2013 ; 2015)$ introduces the concept that the relationship between the indirect effect in a mediation model and the moderator variable is a mathematical function that can be tested directly, and offers this as a simple, accessible, and conceptually and mathematically accurate means of assessing moderated mediation. He denotes this 
function as $\omega$, with the function for a simple mediation model with the first stage path $(X \rightarrow M)$ moderated by $W$ given as:

$$
\omega=\left(a_{1}+a_{3} W\right) b
$$

where $a_{1}$ is the path from $X$ to $M$, and $a_{3} W$ is the path from $W$ to $M$. The sum of these two path coefficients is multiplied by $b$ since the indirect effect in a mediator model is quantified as the product of the first stage $(X \rightarrow M)$ and second stage $(M \rightarrow Y)$ paths (see Equation 2). An equivalent form of Equation 7 appears as:

$$
\omega=a_{1} b+a_{3} b W
$$

which can be graphed as a line where $a_{1} b$ is the intercept and $a_{3} b$ is the slope, or the quantification of the effect of $W$ on the indirect path of $X$ on $Y$ through $M$. If the coefficient $a_{3} b$ is significantly different from zero, it is plausible that $W$ moderates the indirect effect of $X$ on $Y$ through $M$. This can be extended to accommodate more complex models, including the model depicted in Figure 17, by incorporating the appropriate terms from Equations 5 and 6, as given by:

$$
\omega=\left(a_{1}+a_{3} W\right)\left(b_{1}+b_{2} W\right)
$$

which extends to:

$$
\omega=a_{1} b_{1}+a_{3} b_{1} W+a_{1} b_{2} W+a_{3} b_{2} W^{2}
$$

where $\omega$ is a line with intercept $a_{1} b_{1}$ and slope $\left(a_{3} b_{1}+a_{1} b_{2}+a_{3} b_{2}\right)$. Just as with 
Equation 8, the slope of this line is the quantification of the relationship between the moderator $W$ and the size of the indirect path of $X$ on $Y$ through $M$, which can be tested to determine whether it is significantly different from zero, indicating the plausibility of moderation of the mediated pathway. Hayes $(2013 ; 2015)$ has named this function the "Index of Moderated Mediation," and provides a direct test of its significance via bootstrap confidence intervals that is easily accessible through common statistics packages such as Mplus, SAS, and SPSS via programming, which he makes freely available.

PROCESS macro. For SPSS users, this programming is available in the form of a macro named PROCESS ${ }^{1}$. The macro allows for the specification of any one of 79 different path models, ranging from a simple mediator or moderator model, to models that incorporate up to ten mediators and ten moderators, in a variety of configurations. Various other parameters can be specified, including the number of bootstrap iterations desired to produce the confidence intervals for each estimate. The output contains the regression results from Equation 6 and Equation 7, as well as the test statistics for the conditional direct and indirect effects. The covariance matrices can be obtained as an option, and the test of the index of moderation is given. Standard errors and the bootstrap confidence intervals are all included as part of the output.

${ }^{1}$ The PROCESS macro and accompanying documentation can be freely downloaded at www.andrewhayes.com . 
Conditional effects in the present study. The path model tested in the present study is depicted in Figure 17. This model illustrates the inclusion of interaction terms with the moderator (grade) for both the IV (a way of coping at either time 1 or time 2) and the mediator variable (Engagement at time 2), meaning the model includes moderation of all pathways in the mediation model. Achievement is the DV of interest in the present analysis, and is an averaged score of several achievement scores obtained for each student. As such, it is treated as a time 2 measure. To test for age differences in mediational relationships, the PROCESS macro developed by Hayes $(2013 ; 2015)$ for SPSS was used for each model tested by applying the macro to each of the 40 imputed datasets individually, then combining the results according to Rubin's Rules (Rubin, 1987) to obtain a single set of results.

The next section presents the results of the specific analyses conducted to test the hypotheses summarized in Table 6 in Chapter 3 (pp. 128-130). Figures 18, 20, 22, and 23 (pp. 192, 206, 215, and 219, respectively) highlight the specific pathways in the model from Figure 4 (p. 31) that each research question addresses. 


\section{Analyses by Research Question}

1. Are there age-graded differences in the relationships between ways of coping (mastery-oriented and helplessness), engagement, and academic achievement?

$\underline{\text { CONTEXT }} \underline{\text { SELF }} \underline{\text { ACTION }}$

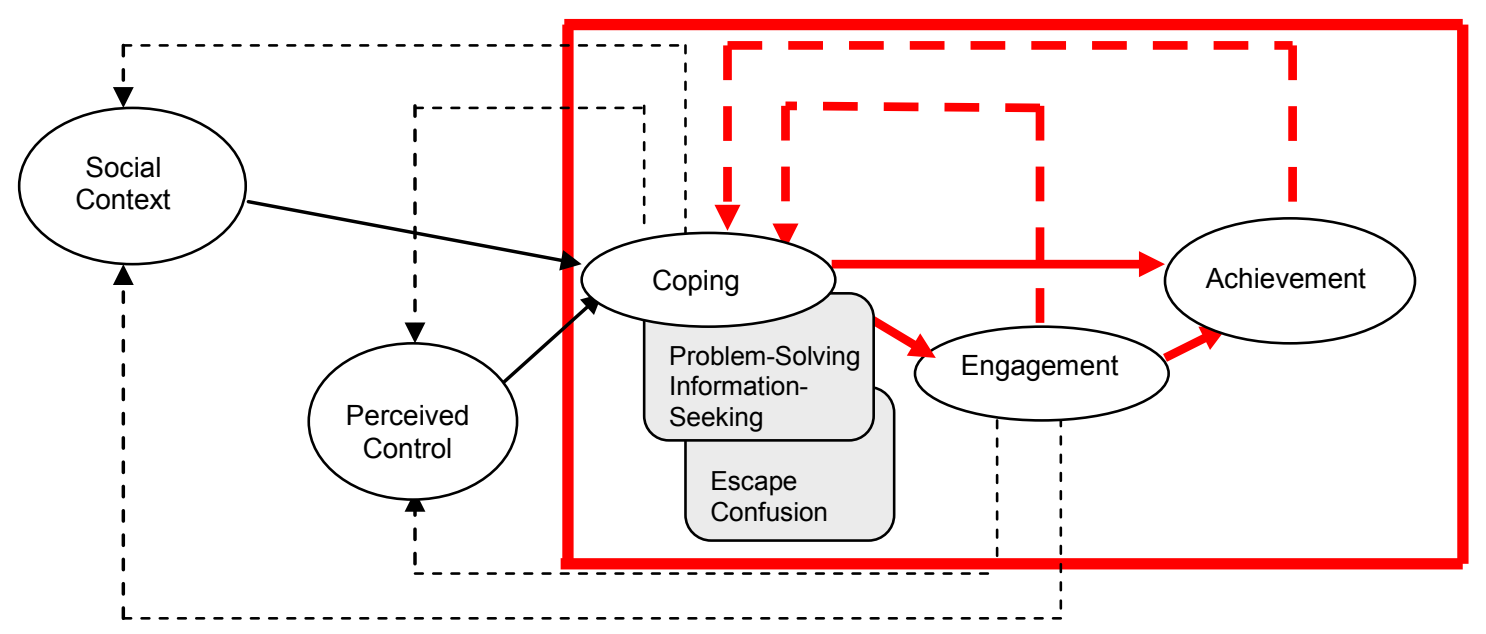

Figure 18: Specific pathways of the overall model addressed by Research Question 1.

This research question proposes six different hypotheses regarding the pathways highlighted in Figure 18. Specific hypotheses were outlined in Table 6 (p. 128-130).

1a) Coping and Engagement. The first three hypotheses address the predicted age differences in the feedforward and feedback relationships between coping and engagement, and changes in engagement, as illustrated by the causal loop diagram in Figure 10 (p. 112). All three hypotheses predict significant age differences.

Correlations - feedforward and feedback effects. The concurrent interconstruct correlations presented in Tables 17-19 (pp. 236-239) show the expected 
pattern of positive and negative correlations between mastery and helplessness ways of coping (respectively) and overall Engagement, indicating that children's increased use of Problem-Solving and Information-Seeking as methods for dealing with academic challenges correspond with stronger Engagement with classroom activities, while increases in Escape behaviors and Confusion are associated with weaker Engagement over time. Also consistent with expectations, the strength of the correlations for mastery ways of coping at both time points was stronger for the $6^{\text {th }}$ grade students, particularly in the fall. Contrary to expectations, Escape coping was stronger for $6^{\text {th }}$ graders than for $4^{\text {th }}$ graders in both the fall and spring, suggesting that $6^{\text {th }}$ grade students' use of avoidance coping is maintained at higher levels than expected across time. Confusion coping was stronger for $4^{\text {th }}$ grade students in both the fall and spring.

The overall correlations for the full sample show that the correlations are generally stronger in the spring than in the fall, suggesting that these behavior patterns are strengthened as children age. The correlations by individual grade, however, show that this pattern holds only for the $4^{\text {th }}$ grade students. The $6^{\text {th }}$ grade students show an opposite pattern, with the overall strength of the correlations between coping and Engagement slightly weaker in the spring, with the exception of Escape coping, which was stronger in the spring than in the fall.

Age differences in correlations - changes in Engagement. Table 21 (pp. 242246) presents the results of the regressions testing the significance of the difference in 
the fall-to-spring correlations between the grades on each construct. The interaction term for Engagement was non-significant, indicating that the amount of change in the construct for $4^{\text {th }}$ graders was not significantly different than the amount of change in Engagement for the $6^{\text {th }}$ graders. This suggests that while analyses may reveal significant age differences in the feedforward and feedback effects between ways of coping and Engagement, detecting any significant age difference in the prediction of changes in Engagement by ways of coping may prove more difficult.

Coping predicting Engagement. To test for age differences in the feedforward pathway from ways of coping to Engagement, simultaneous multiple regressions were conducted using the fall measure of a way of coping, the grade variable (proxy for age), and the cross product for grade * coping as the IVs, and the spring measure of Engagement as the DV. This follows the same structure as the concurrent measures model illustrated in Figure 12 (p. 171), with the modification of using the time 2 measure of the DV rather than the time 1 measure. This was done in order to be consistent with the subsequent mediation models that were tested, so as to provide supporting analytic evidence for the results and interpretation of the mediation analyses. Results of these regressions are presented in Table 23 (p. 248).

For all ways of coping, the direct effects of both coping and grade were significant, indicating that each way of coping is a significant predictor of Engagement, and that $4^{\text {th }}$ grade and $6^{\text {th }}$ grade students differ significantly on the mean of spring Engagement when coping is at zero. Specifically, all coefficients were 
negative, indicating that the mean of Engagement in the spring is significantly lower for $6^{\text {th }}$ grade students than for $4^{\text {th }}$ grade students, when each way of coping is at zero. None of the interaction terms were significant, indicating there are no significant age differences in the prediction of Engagement by coping. The overall models accounted for a significant amount of variance in spring Engagement ranging from 15\% to $33 \%$.

Coping predicting changes in Engagement. To test for significant age differences in the prediction of changes in Engagement by ways of coping, hierarchical multiple regressions were conducted for each way of coping, following the structure of the model depicted in Figure 13 (p. 179), with the fall measure of Engagement entered in the first step of the model, followed by a fall way of coping, the grade variable, and their cross product in step two. The results of these regressions are presented in Tables 24-25 (p. 286-287).

The first step of the model for all ways of coping was significant, with the fall measure of Engagement accounting for $56 \%$ of the variance in the corresponding spring measure when coping is at zero $\left[\mathrm{R}^{2}=.56, \mathrm{~F}(1,663)=\right.$ range: $703.022-$ 968.907, $\mathrm{p}<.001]$. This reflects the relative stability of the construct over time. In the second step of the models, the direct effect for any way of coping was not significant after controlling for fall levels of Engagement, indicating coping does not predict changes over time in Engagement. The grade variable was significant only for Information-Seeking and Confusion coping, with a negative coefficient for both, indicating that after controlling for initial levels of Engagement, $6^{\text {th }}$ grade students 
have a significantly lower mean on Engagement than $4^{\text {th }}$ grade students in either case when Information-Seeking or Confusion is at zero.

None of the interaction terms in any of the models was significant, indicating that after controlling for initial levels of Engagement in the fall, there are no age differences in the prediction of changes in Engagement by coping. In all models, the $\Delta \mathrm{R}^{2}$ statistic had a significant associated F-test, indicating that for each way of coping, the second step of the model as a whole accounted for a significant amount of additional variance in spring Engagement, over and above the amount accounted for in the first step. The additional amount of explained variance ranged from $3 \%$ to $4 \%$.

Feedback effects - Engagement predicting Coping. To test for significant age differences in the feedback effect of Engagement on ways of coping, simultaneous multiple regressions were conducted following the model depicted in Figure 12 (p. 171), with Engagement in the fall, grade, and their cross-product entered as IVs, and a way of coping in the spring entered as the DV. Results of these regressions are presented in Table 26 (p. 251).

In all four models, the direct effect of Engagement was significant, indicating that Engagement has significant feedback effects on all ways of coping. The direct effect for grade was significant only for Problem-Solving and Escape, indicating that $6^{\text {th }}$ grade students differ significantly on the mean of these ways of coping from $4^{\text {th }}$ grade students when Engagement is at zero. The coefficient in the model for ProblemSolving was negative, and the coefficient in the model for Escape was positive, 
meaning that $6^{\text {th }}$ grade students have a significantly lower mean on Problem-Solving and a significantly higher mean on Escape coping than $4^{\text {th }}$ grade students when Engagement is at zero. None of the interaction terms were significant, indicating there are no age differences in the feedback effects of Engagement on ways of coping. The overall models accounted for a significant amount of variance in coping in the spring, ranging from $4 \%$ to $35 \%$.

\section{1b) Coping and Achievement.}

This part of the first research question addresses the feedforward and feedback effects between coping and achievement. There are two hypotheses proposed, each predicting age differences in the pathways relating these constructs.

Correlations - Coping and Achievement. As shown in Tables 17-19 (pp. 236239), all ways of coping are related to achievement at some time point for at least one of the grades. Fourth grades students show no significant relationships in the fall between mastery ways of coping and Achievement, but in the spring this relationship has become significant, and is moderately strong and positive. A strong significant negative correlation is seen in the fall for Escape coping, and a weak but significant positive relationship for Confusion coping. A slight gain in strength is seen for Escape coping in the spring, while Confusion coping drops to non-significance.

Sixth grade students start off in the fall with positive weak, but significant, correlations for the mastery ways of coping, both of which decrease in the spring; Information-Seeking becomes non-significant. Escape coping is strong and negative 
in the fall and decreases slightly in the spring, while Confusion, which was negative, weak, and significant in the fall, changes to a positive, weak, and significant relationship in the spring. These patterns are relatively within expectations for younger children, but unexpected for older children, suggesting that further investigation is warranted to understand the patterns of influence at work directly or indirectly between coping and Achievement.

Coping predicting Achievement. To test for age differences in the prediction of Achievement by coping, simultaneous multiple regressions were conducted, according to the model depicted in Figure 12 (p. 171). Results of these regressions are presented in Table 27 (p. 252). Of the eight regression models that were tested (all four ways of coping in the fall and spring), only Escape showed a significant direct effect on Achievement, at both the fall and spring time points, with a negative coefficient in both instances, indicating that increased use of Escape coping predicts declines in overall Achievement across the year.

A direct effect for grade was seen only in the model for Escape in the spring, with a positive coefficient, indicating that $6^{\text {th }}$ grade students have a significantly higher mean on Achievement in the spring than $4^{\text {th }}$ grade students, when Escape coping is zero. None of the interaction terms for any of the ways of coping were significant, indicating there are no age differences in the prediction of Achievement by coping. Only the models for Escape were shown to account for a significant amount of variance in Achievement at both time points, ranging from $5 \%$ in the fall to $7 \%$ in 
the spring. All other models had a range for the $\mathrm{R}^{2} \mathrm{~F}$-statistic that included at least one non-significant result.

Feedback effects of Achievement on Coping. To test for age differences in the feedback effects of Achievement on ways of coping, simultaneous multiple regressions were conducted following the model in Figure 12 (p. 171), with measures for overall Achievement, grade, and their cross-product as the IVs, and each way of coping in the spring as the DVs. Regression results are presented in Table 28 (p. 253).

Only the model for Escape coping showed a significant direct effect for Achievement, with a negative coefficient. This indicates that Achievement has a significant, negative feedback effect on Escape coping such that as Achievement increases, the use of Escape coping decreases. The grade variable was significant in all models except Confusion coping, with a negative coefficient for mastery ways of coping, and a positive coefficient for Escape coping. This indicates that $6^{\text {th }}$ grade students have a significantly lower mean on mastery coping and a significantly higher mean on Escape coping than $4^{\text {th }}$ grade students, when Achievement is at zero. None of the interaction terms were significant, indicating there are no age differences in the feedback effects of Achievement on any way of coping.

For all models of coping except Confusion coping, a significant amount of variance in coping was explained by the model, ranging from $3 \%$ to $14 \%$. A definitive determination of significance for the $\mathrm{R}^{2}$ value cannot be made for the model for 
Confusion coping because the range of values of the F-statistic contained at least one non-significant result.

1c) Engagement as a mediator in the relationship between coping and achievement.

One hypothesis is proposed for this part of the research question, that Engagement will fully mediate the effects of all ways of coping on Achievement, with no age differences detected.

Models tested. Eight mediation models (four ways of coping at the fall and spring measurement points) were tested to determine if age differences exist in the strength of the indirect influence of a way of coping in either the fall or spring $(\mathrm{X})$ on overall Achievement (Y) through a mediator, spring Engagement (M). This represents a departure from testing only the original three models deemed to provide significant mediation effects, as suggested by this researcher's unpublished Master's thesis. The rationale for this departure is based on the literature reported earlier in this dissertation that describes recent advances in methodologies for testing mediational models that include moderator variables which were not easily accessible when the current study hypotheses were formulated. In light of this information, which highlights the mathematical inaccuracies of excluding models from hypothesis testing based on inference of statistical significance of component pathways in the full mediation model, it is reasonable to consider all possible models for hypothesis testing. 
Additional evidence to support this decision is provided by the results of preceding analyses that confirm significant pathways for all ways of coping to Engagement. Further, the correlations as presented in Tables 17-19 (pp. 236-239) are weak for coping and Achievement, but show moderate to strong correlations between coping and Engagement; this pattern holds at both time points. This suggests that mediation of the influence of ways of coping on Achievement by Engagement is plausible for all possible models. The original stated hypotheses of the current study, that full mediation is expected for the three specified models, with no significant age differences, is extended to the five additional models tested.

Analysis of mediation. Tables 29-36 (p. 254-261) present the estimated path coefficients for each of the eight models tested obtained from the analyses performed using Hayes' PROCESS macro for SPSS (Hayes, 2013; 2015). For the first- stage pathway, $(X \rightarrow M)$, all ways of coping in the fall and spring were significantly related to spring Engagement, in the expected directions, indicating that coping predicts Engagement when all other predictors in the model are zero. All coefficients for mastery coping were positive, and all coefficients for helplessness coping were negative, indicating that as mastery coping increases, Engagement increases, and as use of helplessness coping increases, Engagement decreases.

A significant direct effect for the grade variable was obtained for all models except Escape in the fall, and all significant coefficients were negative, indicating that for all ways of coping except Escape in the fall, $6^{\text {th }}$ grade students have significantly 
lower means for Engagement than $4^{\text {th }}$ grade students when all other predictors in the model are at zero. A significant, positive interaction term for this pathway was noted for both mastery ways of coping in the fall, but not in the spring, and not for either of the helplessness ways of coping. This indicates that when coping, Engagement, and Achievement are included together in the model, the magnitude of the increases in Engagement as mastery coping increases is greater for the $6^{\text {th }}$ grade students, as illustrated by the graphs of these interactions depicted in Figure 19. This step of the model accounted for a significant amount of variance in Engagement in all models, ranging from $17 \%$ to $45 \%$ in the fall, and $24 \%$ to $46 \%$ in the spring.

The second-stage pathway from spring Engagement to Achievement $(\mathrm{M} \rightarrow \mathrm{Y})$ showed a significant and positive direct effect for Engagement in all models except the

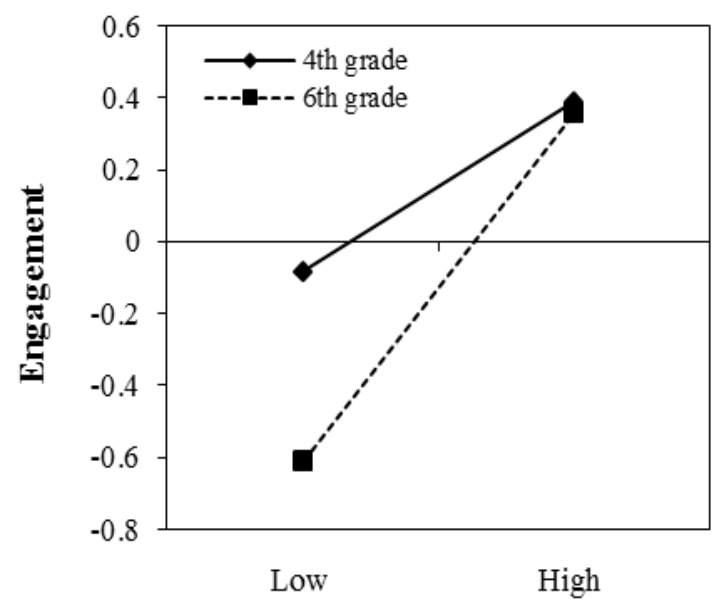

Problem-Solving (fall)

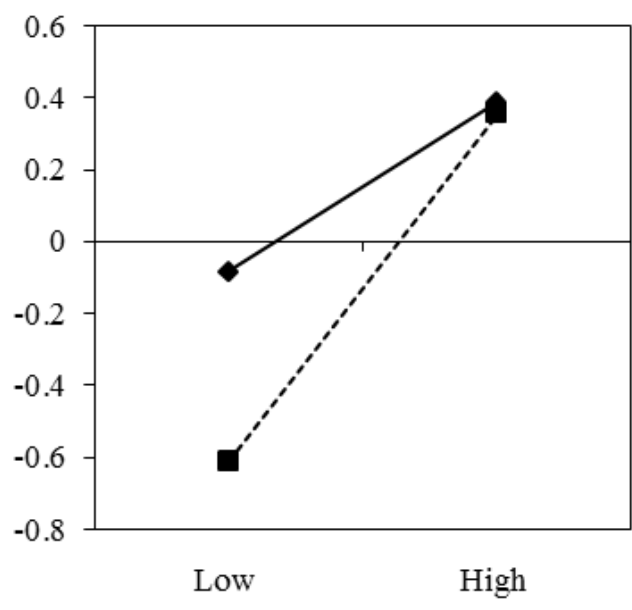

Information-Seeking (fall)

Figure 19: Grade * Coping interaction graphs for mastery ways of coping in the fall predicting spring Engagement when Achievement is in the model. 
model for Escape in the spring, indicating that Engagement is a significant predictor of Achievement when any way of coping included in the model is at zero, with the exception of Escape coping in the spring.

A significant and positive direct effect for grade was noted in the models for Problem-solving, Escape, and Confusion, all in the spring, indicating that students in the $6^{\text {th }}$ grade have a significantly higher mean on Achievement in the spring than $4^{\text {th }}$ grade students, when these ways of coping are included in the model with Engagement and are at zero. The interaction term for the pathway between Engagement and Achievement when coping is included in the model was not significant for any way of coping. This step of the model accounted for a significant amount of variance in Achievement in all models, ranging from $8 \%$ to $9 \%$ in the fall, and $6 \%$ to $8 \%$ in the spring.

For all models except Escape in the spring, both first- and second-stage pathways were significant. No model except Escape in the spring showed a significant direct effect of coping on Achievement. These findings suggest that for all ways of coping except Escape in the spring, Engagement in the spring fully mediates the effect of coping on Achievement in both the fall and the spring. This can be confirmed by a direct test of the indirect path via bootstrap confidence intervals, with significance indicated by a confidence interval that does not contain zero. Results of this test of the coefficients for the indirect effects for each grade, derived from the 
regression coefficients of the tests of the eight mediation models in the preceding analyses, are given in Table 37 (p. 262).

For all ways of coping except Information-Seeking in the spring for $4^{\text {th }}$ graders, Escape coping in the fall for $6^{\text {th }}$ graders, and Escape coping in the spring for both grades, the indirect path from coping to Achievement through Engagement is significant, as indicated by the associated confidence intervals that do not contain zero, indicating the indirect effect for each model is significantly different than zero, thus providing partial support for the hypothesis that Engagement mediates the effect of coping on Achievement for all ways of coping. The exception is Escape coping in the spring, for which no significant mediating effect was found. Mediation of the effect of Information-Seeking in the spring and Escape coping in the fall appears to be conditional on grade level; however, a formal test of moderation is necessary to determine whether the difference between the grades for the indirect effect associated with these ways of coping is significant.

Moderation of conditional effects. In some instances, once having established that mediation exists in general in a model, a conclusion is made about whether moderation of the mediated effect exists, drawn from an examination of the moderated status of each of the component paths. If at least one pathway is moderated, often the inference is made that the entire model and the effect it represents is moderated as well; however, because neither of the pathways alone quantifies the indirect effect of the model, this is an erroneous conclusion. It is the product of the coefficients of the 
first- and second-stage paths that provide the quantification of the indirect effect, and a test of the relationship between the size of this effect and the moderator is needed to determine whether a claim of moderation of the conditional effect is plausible.

Hayes $(2013 ; 2015)$ provides the justification and mathematics for such a test, named by Hayes as the Index of Mediated Moderation (IMM). Using the regression coefficients obtained from the tests of the eight mediation models in the preceding analyses, Equation 10 yields the quantification of the relationship between the indirect effect and the moderator, for each model at each level of the moderator. Because the moderator is a dichotomous variable, the simple difference between the slopes of the lines described by the equation is in fact the IMM, and the subject of the test. The moderator variable is dummy coded with values of 0 and 1 , with $4^{\text {th }}$ grade as the reference group (value of 0 ). The slope for the $4^{\text {th }}$ graders then will be equal to zero, and the test of the null hypothesis equates to a test of whether the slope of the line for the $6^{\text {th }}$ graders describing the relationship between the indirect effect and the moderator (the IMM) is significantly different from zero (the slope of the line for $4^{\text {th }}$ graders).

Table 38 (p. 263) presents the coefficient estimates, standard errors, and bootstrap confidence intervals given by the PROCESS macro, based on 5000 random draws from the sample data, with replacement (resampling). Every confidence interval obtained contains zero, indicating that a value of zero for the estimate of the relationship between the moderator and the indirect path cannot be definitively ruled 
out, and the null hypothesis that the statistic of interest is not significantly different from zero cannot be rejected for any model at either time point. Hence, there is not sufficient evidence of a significant effect for age on the mediated path of the influence of coping on Achievement through Engagement for any way of coping.

2. Do the unique and predictive relationships between the components of perceived control and coping change with age?

\section{$\underline{\text { CONTEXT }} \underline{\text { SELF }} \underline{\text { ACTION }}$}

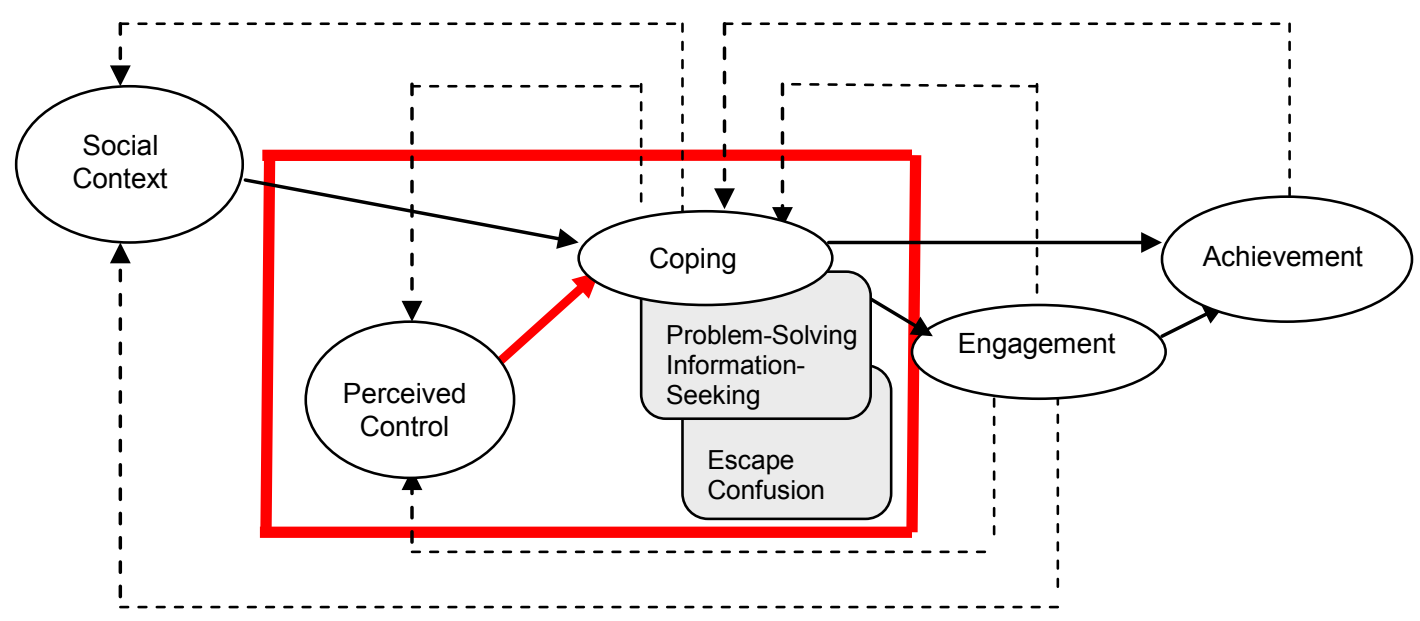

Figure 20: Specific pathways of the overall model addressed by Research Question 2.

Previous research has documented the tripartite structure of perceived control (See Skinner, 1999 for a thorough review). Five hypotheses are proposed for this research question. Age differences are expected to show stronger relationships for younger children between Total Strategy Beliefs and Problem-Solving $(+)$ and Escape 
(-), as well as between CONMAX and Escape (-); stronger relationships for older children are expected between Total Capacity Beliefs and Information-Seeking $(+)$ and Total Strategy Beliefs and Confusion (+). Additional hypotheses regarding the relationships between specific components of control and coping where significant age differences for the aggregate control constructs are found are also proposed and detailed in Table 6 (pp. 128-130).

Analyses using concurrent measures were conducted in the same pattern as established by Skinner, Wellborn \& Connell (1990), and Skinner, et al. (1998) employing first the aggregates for the total effects of strategy and capacity beliefs, followed by analyses using the ten component measures of perceived control where significant age differences for the aggregates are found.

Components of control related to coping, concurrently. Tables 17-19 (pp. 236239) present the inter-construct correlations for the full sample and for each grade individually. Total Strategy Beliefs are significantly and negatively correlated with mastery ways of coping, and significantly and positively correlated with helplessness ways of coping for both grade groups. Correlations between these constructs are moderate for $6^{\text {th }}$ grade students, and low for $4^{\text {th }}$ grade students. Total Capacity Beliefs shows the opposite pattern for both groups- significant positive correlations with mastery ways of coping and significant negative correlations with helplessness ways of coping. 
Because the correlations between Total Capacity Beliefs and CONMAX are so high, the analysis of correlations in this next section applies to both constructs. The correlations between these constructs and the four ways of coping show all correlations to be significant, with moderate positive correlations with the mastery ways of coping, and strong negative correlations with helplessness ways of coping. Correlations are stronger for $6^{\text {th }}$ grade students for all ways of coping except Confusion coping, which shows a stronger correlation with control for $4^{\text {th }}$ grade students. All correlations for $4^{\text {th }}$ grade students were stronger in the spring than in the fall, but for $6^{\text {th }}$ grade students the pattern differs not only from the $4^{\text {th }}$ grade students, but by control construct as well. For Total Capacity Beliefs, $6^{\text {th }}$ grade students show a stronger correlation over the fall only for Escape coping. The correlation for all other ways of coping declined slightly or stayed the same. For CONMAX, only the correlations with Information-Seeking and Confusion declined in the spring; ProblemSolving and Escape both showed a slight increase. Overall, the correlation with Escape coping is the strongest correlation for both grade groups at both time points.

To test for significant age differences in the prediction of coping by control, simultaneous regressions were conducted following the model depicted in Figure 12 (p. 171). For each way of coping, separate regressions were conducted for the Total Strategy and Capacity Beliefs control aggregates at each time point for a total of four regressions for each way of coping (two aggregates by two time points), with a control aggregate, the grade variable, and their cross product entered as IVs, and a concurrent 
way of coping as the DV. Results of these regressions are presented in Tables 39-40 (pp. 264-265).

Overview. The direct effect for both control aggregates was significant in every model, indicating that the control aggregates are significant predictors of each way of coping at both time points, in the expected directions. Increases in Total Strategy Beliefs are negatively related to increases in mastery coping and positively related to increases in helplessness coping, while Total Capacity Beliefs demonstrate the opposite relationships.

Total Strategy Beliefs. The direct effect for the grade variable was significant for Total Strategy Beliefs for all ways of coping except Confusion at both time points, indicating a significant difference between the grades on the means of mastery ways of coping and Escape coping when Total Strategy Beliefs is at zero. At both time points, coefficients were negative for mastery ways of coping and positive for Escape coping, indicating that $6^{\text {th }}$ grade students have a lower mean than $4^{\text {th }}$ grade students on both mastery ways of coping when Total Strategy Beliefs are zero, but a higher mean on Escape coping. As Total Strategy Beliefs increase, mastery coping decreases, and Escape coping increases.

The interaction terms for both mastery ways of coping were significant in the fall, but not in the spring, and not for helplessness ways of coping at either time point. This indicates there are significant age differences in the prediction of mastery coping by Total Strategy Beliefs at the fall measurement point. The coefficients for both 
ways of coping were negative, indicating that as Total Strategy Beliefs increase, decreases in mastery ways of coping are larger for $6^{\text {th }}$ grade students than for $4^{\text {th }}$ grade students. Graphs of these interactions are depicted in Figure 21.

Total Capacity Beliefs. The direct effect for the grade variable for Total Capacity Beliefs was significant only for Escape coping in the spring, indicating that when Total Capacity Beliefs is at zero, there is a significant difference between the grades on the mean of Escape coping. The coefficient was positive, indicating that $6^{\text {th }}$ grade students have a higher mean on Escape coping than $4^{\text {th }}$ grade students when Total Capacity Beliefs is at zero. As Total Capacity Beliefs increase, Escape coping also increases. None of the interaction terms were significant, indicating there are no age differences in the prediction of coping by Total Capacity Beliefs.

All models accounted for a significant amount of variance in coping, ranging
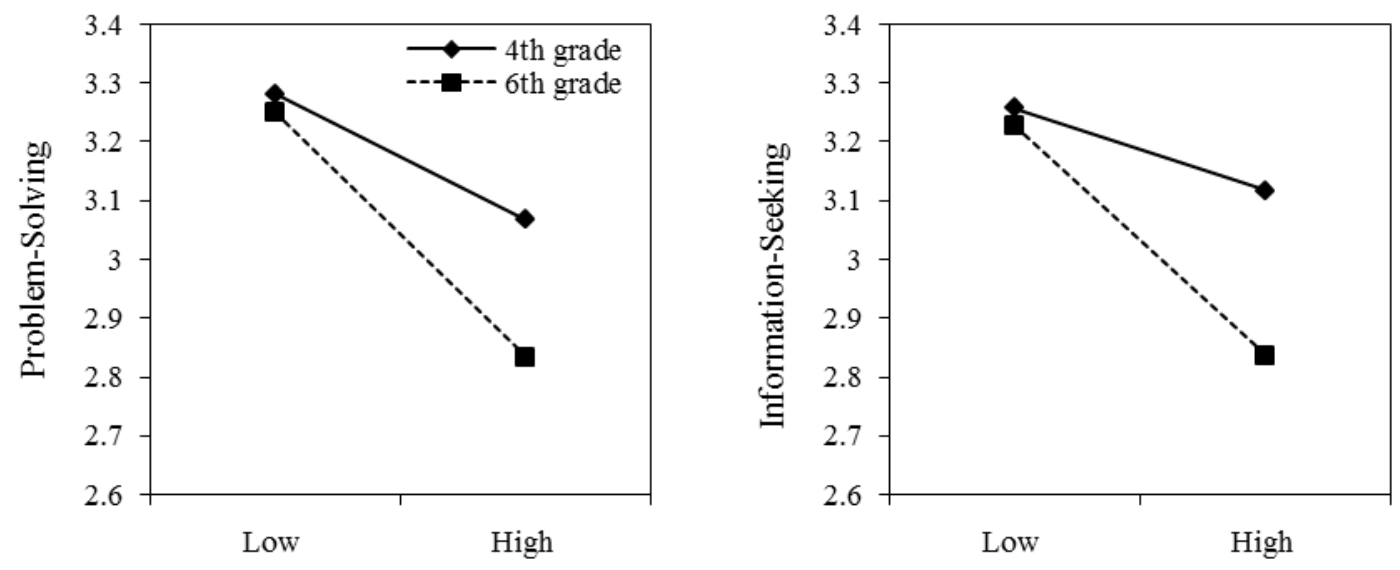

Total Strategy Beliefs (fall)

Figure 21: Grade* Control interaction graphs for Total Strategy Beliefs in the fall predicting mastery ways of coping, concurrently. 
from $3 \%$ to $20 \%$ in the fall, and $4 \%$ to $34 \%$ in the spring for Total Strategy Beliefs, and from $13 \%$ to $39 \%$ in the fall, and $15 \%$ to $40 \%$ in the spring for Total Capacity Beliefs.

Disaggregation of Total Strategy Beliefs. A significant interaction was found for the prediction of mastery ways of coping by Total Strategy Beliefs in the fall. Analyses to disaggregate the Total Strategy Beliefs aggregate into its component constructs provides more information regarding which component is contributing most to the difference in prediction. These analyses followed the same simultaneous regression model for concurrent measures as depicted in Figure 12 (p. 171) that was used for the analysis of the control aggregates.

Problem-Solving. Regression results for the disaggregated components of Total Strategy Beliefs predicting Problem-Solving in the fall are presented in Table 41 (p. 266). A significant direct effect is obtained for Effort, indicating this particular control strategy is a significant predictor of Problem-Solving coping. As belief in Effort as a strategy for success increases, use of Problem-Solving coping strategies also increases. None of the other strategy beliefs were significant. A significant direct effect for grade was also obtained, meaning that the two grade groups differ significantly on the mean of Problem-Solving when all other predictors in the model are at zero. The coefficient was negative, indicating that $6^{\text {th }}$ grade students have a lower mean on Problem-Solving in the fall when all strategy beliefs are at zero. None of the interaction terms for this model were significant, indicating there are no age 
differences in the predictive relationship between Strategy Beliefs for Effort, and Problem-Solving in the fall.

Information-Seeking. Regression results for the disaggregated components of Total Strategy Beliefs predicting Information-Seeking in the fall are presented in Table 42 (p. 267). No significant effects are noted for any of the strategy beliefs individually, indicating that the predictive power of the aggregate construct is carried in the additive effect of all the strategies combined. A significant effect for grade is noted, indicating a significant difference between the two grade groups on the mean of Information-Seeking in the fall, when all strategy beliefs are at zero. The coefficient is negative, indicating that the mean for Information-Seeking in the fall when all other predictors in the model are at zero is lower for $6^{\text {th }}$ grade students than for $4^{\text {th }}$ grade students. None of the interaction terms are significant, indicating there are no age differences in the prediction of this way of coping that can be detected.

Both models significantly accounted for $11 \%$ of the variance each in ProblemSolving and Information-Seeking in the fall.

Control aggregate predicting changes in coping over time. To test for significant age differences in the prediction of changes in ways of coping from fall to spring by control, hierarchical multiple regressions were conducted following the model depicted in Figure 13 (p. 179). For each way of coping, the fall measure of that way of coping was entered into the first step of the regression to account for variance in the spring measure that could be attributed to initial levels of coping. In the second 
step of the model, the control aggregate CONMAX, the grade variable, and their cross-product were entered. Regression results are presented in Tables 43-44 (pp. 268-269).

The first step of the model was significant for all ways of coping, indicating that initial levels of coping account for a significant amount of variance in spring coping levels for all students (Problem-Solving: $\left[\mathrm{R}^{2}=.16, \mathrm{~F}(1,663)=\right.$ range: 73.488 $-171.205, \mathrm{p}<.001]$, Information-Seeking: $\left[\mathrm{R}^{2}=.28, \mathrm{~F}(1,663)=\right.$ range: $187.248-$ 320.406, $\mathrm{p}<.001]$, Escape: $\left[\mathrm{R}^{2}=.31, \mathrm{~F}(1,663)=\right.$ range: $253.157-369.848, \mathrm{p}<$ $.001]$, Confusion: $\left[\mathrm{R}^{2}=.32, \mathrm{~F}(1,663)=\right.$ range: $\left.\left.247.201-395.916, \mathrm{p}<.001\right]\right)$. This reflects the relative stability of these constructs over time.

In the second step of the model, the CONMAX aggregate was also significant for all ways of coping, indicating that after removing variance accounted for by the fall measure of coping, CONMAX is a significant predictor of changes in coping over time. A significant amount of variance was accounted for by the second step of all models, with $\Delta \mathrm{R}^{2}$ ranging from .02 to .08 , indicating that from $2 \%-8 \%$ additional variance in the spring measures of coping was accounted for by the second step of the models. Coefficients were in the expected directions, with positive effects for mastery ways of coping and negative effects for helplessness ways of coping, indicating that as maximum control increases, mastery coping also increases, while helplessness coping decreases. 
The grade variable was significant only for Problem-Solving and Escape coping, indicating that $6^{\text {th }}$ grade students differ significantly from $4^{\text {th }}$ grade students on the means of these ways of coping in the spring when CONMAX is at zero, and after removing the variance in spring coping accounted for by fall measures of coping. The coefficient for Problem-Solving was negative, and for Escape was positive, indicating that when compared to $4^{\text {th }}$ graders, $6^{\text {th }}$ grade students have a lower mean on ProblemSolving, and a higher mean on Escape coping, when CONMAX is at zero and after removing variance in the spring coping measures accounted for by the fall measures of those ways of coping. The interaction terms for all ways of coping were not significant, indicating no age differences in the prediction of changes in coping over time by control, after removing the variance accounted for by the fall measures of coping, can be detected. 
3. Do the unique and predictive relationships between support provided by the social context and coping change with age?

\section{$\underline{\text { CONTEXT }} \underline{\text { SELF }} \underline{\text { ACTION }}$ OUTCOME}

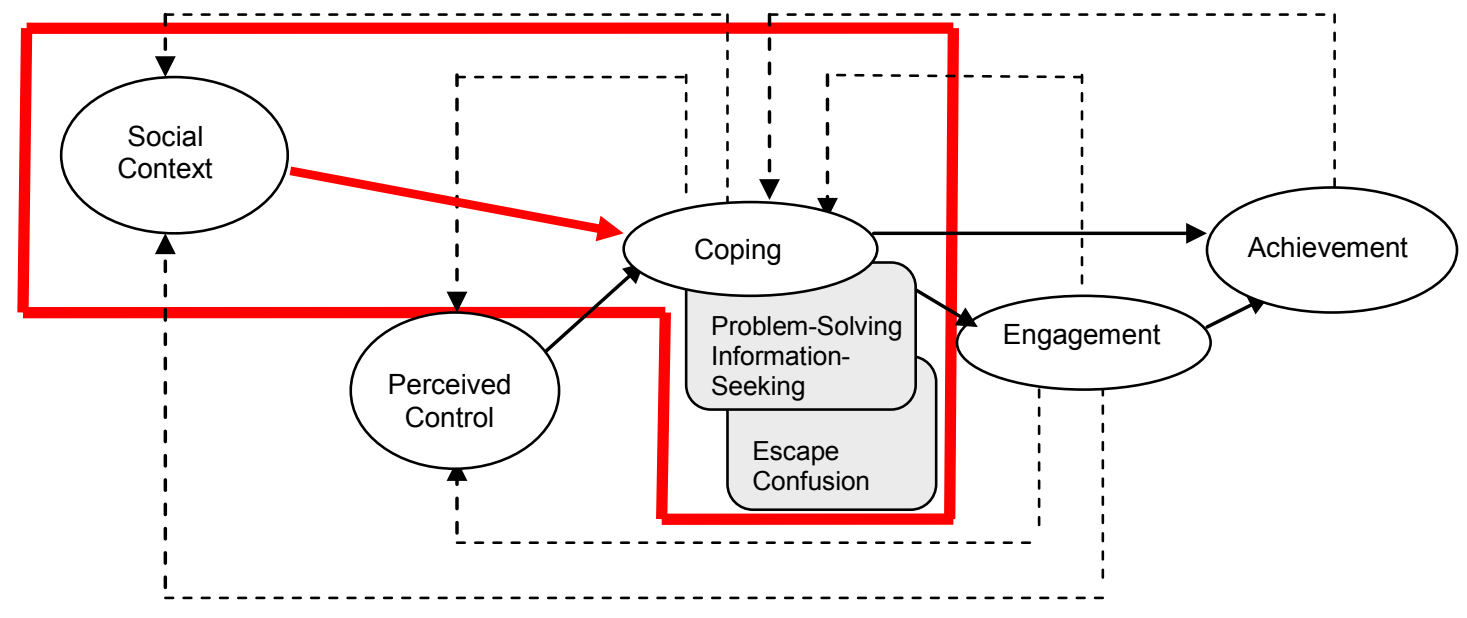

Figure 22: Specific pathways of the overall model addressed by Research Question 3.

The social context of teachers is measured as the extent to which teachers provide structure, involvement, and autonomy support. Due to the lack of association previously found by this researcher in her unpublished Master's thesis for some components of this construct, Teacher Support was tested as an aggregate construct. Four hypotheses are proposed for this research question; three hypotheses detail the direction of influence between coping and teacher support, and for which grade the relationship is strongest. Specifically, the relationships between ways of coping and Total Teacher Support are proposed to be stronger for older children compared to younger children for all ways of coping except Problem-Solving, in the expected 
directions. Problem-Solving is expected to show a stronger positive relationship with teacher support for younger children compared to older children. The fourth hypothesis proposes no age differences in the prediction of changes in coping by Total Teacher Support.

Teacher Support related to ways of coping, concurrently. Examination of the inter-construct correlations presented in Tables 17-19 (pp. 236-239) show weak but significant correlations between the constructs for Total Teacher Support and each way of coping for the overall sample, with correlations generally lower in the fall than in the spring. Most of the association between these variables is being carried by the $6^{\text {th }}$ grade students, as the correlations for the grade groups individually show no correlation between these variables at either time point for the $4^{\text {th }}$ grade students for any way of coping except Escape coping, which is negative and significant. The strength of this particular correlation is similar between the two grade groups, with only a slight increase from fall to spring for either group. All other correlations are weak but significant for the $6^{\text {th }}$ graders. The pattern at both time points shows mastery coping positively related and helplessness coping negatively related to Total Teacher Support, with the exception of Confusion coping in the spring, which shows a weak but positive correlation.

To test for age differences in the prediction of ways of coping concurrently by Total Teacher Support, simultaneous regressions following the model in Figure 12 (p. 171) were conducted. Regression results are presented in Tables 45-46 (pp. 270-271). 
As expected due to the lack of correlation for the $4^{\text {th }}$ graders, Total Teacher Support was not a significant predictor of ways of coping for any way of coping except Escape coping, which was negative, indicating that as teachers offer more support for students, use of Escape coping decreases. The grade variable was significant in the models for mastery ways of coping, and for Escape coping, but not Confusion coping, indicating that $6^{\text {th }}$ grade students differ significantly on the mean of coping from $4^{\text {th }}$ grade students when Total Teacher Support is zero for all ways of coping except Confusion coping. The coefficients for the mastery ways of coping were negative, indicating that $6^{\text {th }}$ graders have a lower mean on these ways of coping than $4^{\text {th }}$ grade students when Total Teacher Support is zero. The coefficient for Escape coping was positive, indicating that $6^{\text {th }}$ grade students have a higher mean on this way of coping than $4^{\text {th }}$ grade students, when Total Teacher Support is at zero. The interaction term was not significant for any way of coping, indicating there are no age differences in the prediction of coping by Total Teacher Support.

All models except the fall model for Problem-Solving, and the models for Confusion at both time points accounted for a significant amount of variance in coping, ranging from $3 \%$ to $9 \%$ in the fall, and $3 \%$ to $13 \%$ in the spring. The models for Problem-Solving in the fall and Confusion at both time points showed at least one non-significant result for the F-statistic for the $\mathrm{R}^{2}$ value, and therefore could not definitively be shown to account for a significant amount of variance in coping overall. 
Teacher Support predicting changes in ways of coping. To test for age differences in the prediction of changes in coping by Teacher Support, hierarchical regressions were conducted following the model depicted in Figure 13 (p. 179). Results of these regressions are presented in Tables 47-48 (pp. 272-273). The fall measure of a way of coping was entered in the first step of the equation to control for variance in the spring measure of that way of coping. Total Teacher Support, the variable for grade, and their cross-product were entered in the second step.

For all ways of coping, the first step of the model was significant, indicating that fall coping accounts for a significant amount of variance in coping in the spring, as previously reported. The direct effect for Total Teacher Support was not significant in any of the models, indicating that after controlling for levels of coping in the fall, Total Teacher Support does not explain any of the remaining variance in coping in the spring. A significant negative effect for grade was noted for Problem-Solving and a significant positive effect for grade was noted for Confusion coping, indicating that $6^{\text {th }}$ grade students differ from $4^{\text {th }}$ grade students on the mean of these ways of coping in the spring when Total Teacher Support is zero, after controlling for fall coping. Specifically, $6^{\text {th }}$ grade students have a lower mean on Problem-Solving and a higher mean on Confusion coping in the spring than $4^{\text {th }}$ grade students when Total Teacher Support is zero, after accounting for initial levels of coping in the fall. None of the interaction terms were significant, indicating no age differences in the relationship of Total Teacher Support to changes in any of the ways of coping. 
4. Are there age differences in the reciprocal effects of coping and engagement on components of perceived control and teacher support?

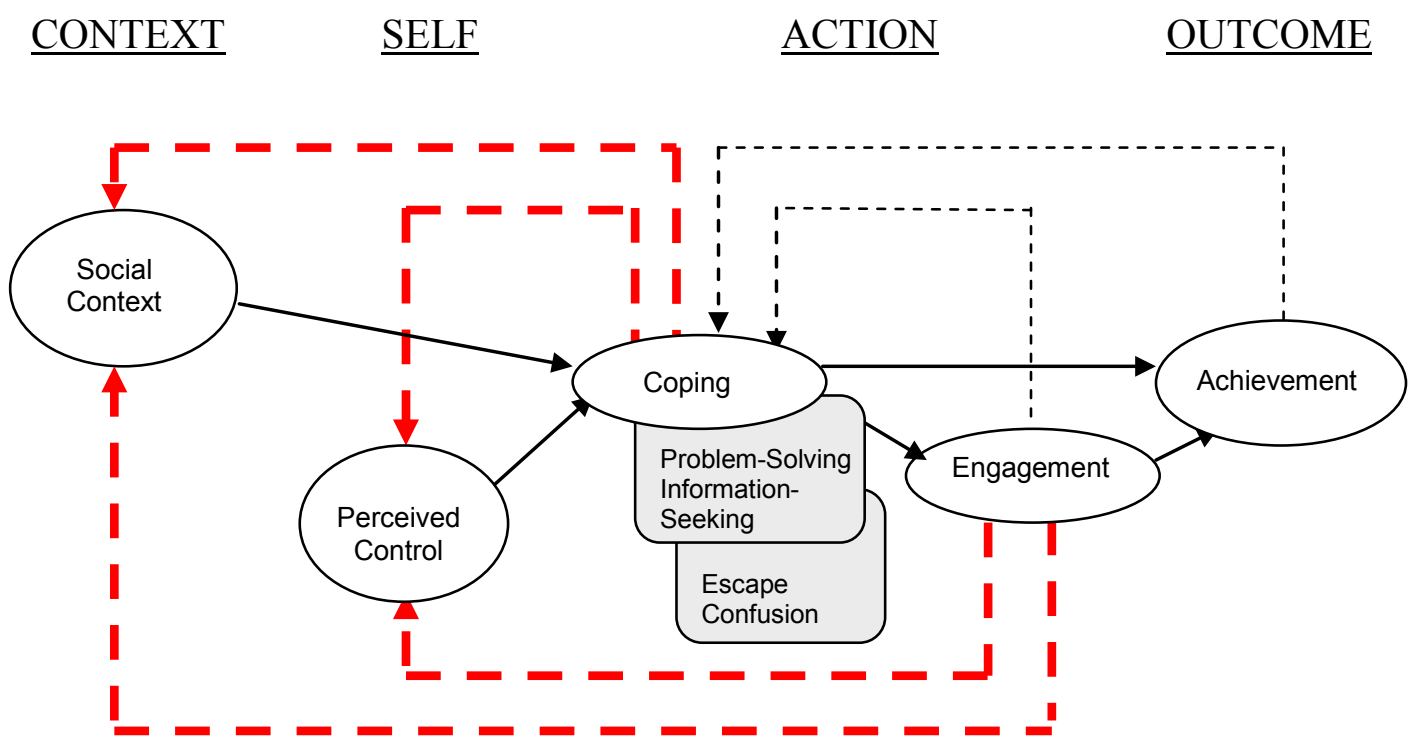

Figure 23: Specific pathways of the overall model (bolded) addressed by Research Question 4.

This set of analyses tested for age differences in the feedback pathways from ways of coping and Engagement to perceived control and the provision of support by teachers to determine whether and how these resources and contextual factors are shaped over time differently with the progression of normative development. Three hypotheses are proposed for this research question, as detailed in Table 6 (pp. 128130). In sum, all feedback pathways are expected to be significantly stronger for older children than younger children, in the expected directions, except for the feedback pathway from Problem-Solving to control. No age differences are expected for this pathway. 
To test for age differences in a reciprocal model, hierarchical regressions were conducted, following the change over time model depicted in Figure 13 (p. 179). Three sets of five regressions each were calculated; one set for each of the three DVs (Total Strategy Beliefs, Total Capacity Beliefs, and Total Teacher Support), with the fall measure of the DV entered in the first step of the equation to control for the contributions of initial levels of that construct. In the second step of the regression, the fall measure for a way of coping or for Engagement, the grade variable, and their cross-product were entered as IVs.

Feedback effects of coping on control. Tables 49-52 (pp. 274-277) present the regression results for the feedback effects of coping on control. The first step of all eight models (four ways of coping by two control aggregates, Total Strategy and Total Capacity Beliefs) was significant, indicating that initial levels of control in the fall accounted for a significant amount of variance in the spring measure of control for all students when coping is at zero (Total Strategy Beliefs: $\left[\mathrm{R}^{2}=.31, \mathrm{~F}(1,663)=\right.$ range: $238.970-344.964, \mathrm{p}<.001]$, Total Capacity Beliefs: $\left[\mathrm{R}^{2}=.45, \mathrm{~F}(1,663)=\right.$ range: $470.702-628.111, \mathrm{p}<.001])$.

The direct effect of coping on control was not significant for any way of coping on either of the control aggregates except for Escape coping on Total Strategy Beliefs. This indicates that after controlling for the contribution of control in the fall, no way of coping had a significant feedback effect on control except Escape coping on strategy beliefs. For this model, the coefficient for coping was positive, indicating that 
higher endorsement of Escape coping predicts increases in Total Strategy Beliefs over time, after controlling for the contribution of initial levels of strategy beliefs to the variance in spring measures of control.

A significant positive direct effect for grade was obtained in all models for Total Strategy Beliefs, and a significant negative direct effect was obtained for all models for Total Capacity Beliefs. This indicates that $6^{\text {th }}$ grade students have a significantly higher mean than $4^{\text {th }}$ grade students on Total Strategy Beliefs when each way of coping is at zero, and a significantly lower mean on Total Capacity Beliefs, when coping is at zero. None of the interaction terms was significant for any of the eight models, indicating no age differences in the feedback effects of ways of coping on control.

Feedback effects of Engagement on control. Table 55 (p. 280) presents the results of the regressions testing the feedback effects of Engagement on the control aggregates. The first step in both equations was significant, indicating that fall levels of control account for a significant amount of variance in the spring measure of control for all students when Engagement is zero, as previously reported. The direct effect for Engagement was significant and negative for Total Strategy Beliefs, and significant and positive for Total Capacity Beliefs, indicating that Engagement has significant feedback effects on control. As Engagement increases, Total Strategy Beliefs decrease, and Total Capacity Beliefs increase. The direct effect for grade was also significant in every model, in the opposite pattern from Engagement - the effect 
was positive for Total Strategy Beliefs, and negative for Total Capacity Beliefs. This indicates that $6^{\text {th }}$ grade students have a significantly higher mean on Total Strategy Beliefs and a significantly lower mean on Total Capacity Beliefs than $4^{\text {th }}$ grade students when Engagement is zero. Neither of the interaction terms was significant, indicating no age differences in the feedback effect of Engagement on control. Feedback effects of coping on Total Teacher Support. Tables 53-54 (pp. 278279) present the results of the regressions testing age differences in the feedback effects of coping on Total Teacher Support. The first step for all models was significant, indicating that initial levels of teacher support in the fall accounted for a significant portion of the variance in the spring measure of teacher support for all students $\left(\mathrm{R}^{2}=.59, \mathrm{~F}(1,663)=\right.$ range: $\left.899.982-1005.618, \mathrm{p}<.001\right)$. No way of coping showed a direct effect on Total Teacher Support, indicating that after controlling for initial levels of teacher support there are no feedback effects of coping on Total Teacher Support. The direct effect for grade was significant and positive in all models, indicating that $6^{\text {th }}$ grade students have a significantly higher mean level of teacher support than $4^{\text {th }}$ grade students, after controlling for initial levels of teacher support in the fall, and when any way of coping included in the model is at zero. None of the interaction terms were significant, indicating there are no age differences in the feedback effect of coping on Total Teacher Support.

Feedback effects of Engagement on Total Teacher Support. Table 55 (p. 280) presents the results of the regression model testing for age differences in the feedback 
effects of Engagement on Total Teacher Support. The first step of the model was significant, indicating that initial levels of overall teacher support accounted for a significant portion of the variance in the spring measure of teacher support for all students, as previously reported. The direct effect of Engagement on teacher support was not significant, indicating there are no feedback effects of Engagement on Total Teacher Support after controlling for initial levels of teacher support in the fall. The direct effect for grade was significant and positive, indicating $6^{\text {th }}$ grade students have a significantly higher mean level of overall teacher support than $4^{\text {th }}$ grade students, after controlling for initial levels of teacher support, when Engagement is at zero. The interaction term was not significant, indicating no age differences in the feedback effect of Engagement on Total Teacher Support. 


\begin{tabular}{|c|c|c|c|c|c|c|c|c|c|c|c|c|c|}
\hline \multicolumn{14}{|c|}{ Table 8} \\
\hline Scale & $\begin{array}{l}\text { Number } \\
\text { of Items }\end{array}$ & $\begin{array}{c}\text { Fall } \\
\alpha\end{array}$ & $\begin{array}{c}\text { Spring } \\
\alpha\end{array}$ & $\begin{array}{l}\text { Fall } \\
M\end{array}$ & $\begin{array}{c}\text { Spring } \\
M\end{array}$ & $\begin{array}{l}\text { Fall } \\
S D\end{array}$ & $\begin{array}{c}\text { Spring } \\
S D\end{array}$ & g & $\begin{array}{l}\text { Fall } \\
\text { Min }\end{array}$ & $\begin{array}{l}\text { Spring } \\
\text { Min }\end{array}$ & $\begin{array}{l}\text { Fall } \\
\text { Max }\end{array}$ & $\begin{array}{c}\text { Spring } \\
\text { Max }\end{array}$ & $\begin{array}{c}\mathrm{F}-\mathrm{S} \\
\quad r\end{array}$ \\
\hline \multicolumn{14}{|l|}{ Perceived Control } \\
\hline Control Beliefs & 6 & .64 & .69 & 3.41 & 3.37 & .51 & .53 & 1.748 & 1.33 & 1.33 & 4.00 & 4.00 & $.59 * * *$ \\
\hline Total Strategy Beliefs & 20 & .80 & .81 & 1.95 & 1.97 & .34 & .36 & -.945 & 1.09 & 1.10 & 3.13 & 3.04 & $.62 * * *$ \\
\hline Effort & 4 & .62 & .64 & 3.17 & 3.08 & .58 & .64 & -.726 & 1.00 & 1.00 & 4.00 & 4.00 & $.46^{* * *}$ \\
\hline Ability & 4 & .69 & .67 & 2.54 & 2.53 & .73 & .70 & .819 & 1.00 & 1.00 & 4.00 & 4.00 & $.55^{* * *}$ \\
\hline Powerful Others & 4 & .71 & .72 & 1.73 & 1.78 & .66 & .67 & $-2.707 * *$ & 1.00 & 1.00 & 4.00 & 4.00 & $.60 * * *$ \\
\hline Luck & 4 & .69 & .74 & 1.83 & 1.84 & .68 & .69 & -.759 & 1.00 & 1.00 & 4.00 & 4.00 & $.62 * * *$ \\
\hline Unknown & 4 & .64 & .71 & 1.90 & 1.86 & .67 & .67 & .930 & 1.00 & 1.00 & 4.00 & 4.00 & $.57 * * *$ \\
\hline Total Capacity Beliefs & 23 & .89 & .91 & 3.26 & 3.17 & .46 & .49 & $4.930 * * *$ & 1.44 & 1.38 & 4.00 & 4.00 & $.74 * * *$ \\
\hline Effort & 6 & .70 & .72 & 3.34 & 3.25 & .54 & .56 & $5.077 * * *$ & 1.00 & 1.49 & 4.00 & 4.00 & $.64 * * *$ \\
\hline Ability & 6 & .80 & .79 & 3.31 & 3.21 & .55 & .58 & $3.445^{* * *}$ & 1.33 & 1.33 & 4.00 & 4.00 & $.68 * * *$ \\
\hline Powerful Others & 5 & .70 & .75 & 3.31 & 3.22 & .61 & .63 & $2.074 *$ & 1.00 & 1.00 & 4.00 & 4.00 & $.58 * * *$ \\
\hline Luck & 6 & .68 & .73 & 3.08 & 3.01 & .58 & .60 & $3.428 * * *$ & 1.17 & 1.00 & 4.00 & 4.00 & $.60 * * *$ \\
\hline
\end{tabular}

Note: $N=665 ; * p<.05 .{ }^{* *} p<.01 .{ }^{* * *} p<.001$. 


\begin{tabular}{|c|c|c|c|c|c|c|c|c|c|c|c|c|c|}
\hline \multicolumn{14}{|c|}{ Table 8} \\
\hline \multicolumn{14}{|c|}{ Properties of Measurement Scales, Descriptive Statistics, and Stabilities - Full Sample, cont'd } \\
\hline Scale & $\begin{array}{l}\text { Number } \\
\text { of Items }\end{array}$ & $\begin{array}{c}\text { Fall } \\
\alpha\end{array}$ & $\begin{array}{c}\text { Spring } \\
\alpha\end{array}$ & $\begin{array}{c}\text { Fall } \\
M\end{array}$ & $\begin{array}{c}\text { Spring } \\
M\end{array}$ & $\begin{array}{l}\text { Fall } \\
S D\end{array}$ & $\begin{array}{c}\text { Spring } \\
S D\end{array}$ & $t$ & $\begin{array}{l}\text { Fall } \\
\text { Min }\end{array}$ & $\begin{array}{l}\text { Spring } \\
\text { Min }\end{array}$ & $\begin{array}{l}\text { Fall } \\
\text { Max }\end{array}$ & $\begin{array}{l}\text { Spring } \\
\text { Max }\end{array}$ & $\begin{array}{c}\mathrm{F}-\mathrm{S} \\
r\end{array}$ \\
\hline \multicolumn{14}{|l|}{ Coping } \\
\hline Problem-Solving & 5 & .66 & .75 & 3.13 & 3.00 & .55 & .63 & $4.989 * * *$ & 1.00 & 1.00 & 4.00 & 4.00 & $.45^{* * *}$ \\
\hline Information-Seeking & 5 & .72 & .78 & 3.14 & 3.07 & .59 & .62 & $2.252^{*}$ & 1.20 & 1.00 & 4.00 & 4.00 & $.59 * * *$ \\
\hline Escape & 4 & .77 & .72 & 1.65 & 1.74 & .64 & .62 & $-4.688 * * *$ & 1.00 & 1.00 & 4.00 & 4.00 & $.61^{* * *}$ \\
\hline Confusion & 5 & .75 & .77 & 2.27 & 2.26 & .70 & .69 & .900 & 1.00 & 1.00 & 4.00 & 4.00 & $.59 * * *$ \\
\hline Total Engagement & 25 & .89 & .91 & 3.18 & 3.05 & .45 & .44 & $9.571 * * *$ & 1.85 & 1.63 & 4.00 & 3.88 & $.78 * * *$ \\
\hline Behavioral & 10 & .74 & .82 & 3.28 & 3.09 & .48 & .41 & $14.105^{* * *}$ & 1.73 & 1.58 & 4.00 & 3.75 & $.78 * * *$ \\
\hline Emotional & 15 & .86 & .88 & 3.07 & 3.01 & .51 & .54 & $3.258 * * *$ & 1.31 & 1.22 & 4.00 & 4.00 & $.72 * * *$ \\
\hline Total Teacher Support & 37 & .92 & .92 & 3.09 & 3.15 & .36 & .40 & $-4.742 * * *$ & 1.84 & 2.05 & 3.89 & 3.96 & $.78 * * *$ \\
\hline Structure & 7 & .68 & .75 & 3.35 & 3.40 & .51 & .54 & $-2.711 * *$ & 1.33 & 1.69 & 4.00 & 4.00 & $.69 * * *$ \\
\hline Involvement & 16 & .89 & .90 & 3.01 & 3.06 & .44 & .48 & $-3.454 * * *$ & 1.84 & 1.39 & 3.93 & 4.00 & $.79 * * *$ \\
\hline Autonomy Support & 14 & .88 & .89 & 2.92 & 2.98 & .49 & .53 & $-4.104 * * *$ & 1.42 & 1.49 & 4.00 & 4.00 & $.77 * * *$ \\
\hline
\end{tabular}


Table 9

Properties of Measurement Scales, Descriptive Statistics, and Stabilities $-4^{\text {th }}$ Grade Sample

\begin{tabular}{|c|c|c|c|c|c|c|c|c|c|c|c|c|c|}
\hline Scale & $\begin{array}{l}\text { Number } \\
\text { of Items }\end{array}$ & $\begin{array}{c}\text { Fall } \\
\alpha\end{array}$ & $\begin{array}{c}\text { Spring } \\
\alpha\end{array}$ & $\begin{array}{c}\text { Fall } \\
M\end{array}$ & $\begin{array}{c}\text { Spring } \\
M\end{array}$ & $\begin{array}{l}\text { Fall } \\
S D\end{array}$ & $\begin{array}{c}\text { Spring } \\
S D\end{array}$ & $t$ & $\begin{array}{l}\text { Fall } \\
\text { Min }\end{array}$ & $\begin{array}{c}\text { Spring } \\
\text { Min }\end{array}$ & $\begin{array}{l}\text { Fall } \\
\text { Max }\end{array}$ & $\begin{array}{l}\text { Spring } \\
\text { Max }\end{array}$ & $\begin{array}{c}\mathrm{F}-\mathrm{S} \\
r\end{array}$ \\
\hline
\end{tabular}

\section{Perceived Control}

\begin{tabular}{|c|c|c|c|c|c|c|c|c|c|c|c|c|c|}
\hline Control Beliefs & 6 & .61 & .66 & 3.44 & 3.46 & .49 & .51 & -.942 & 1.33 & 1.68 & 4.00 & 4.00 & $.51 * * *$ \\
\hline Total Strategy Beliefs & 20 & .81 & .80 & 1.98 & 1.92 & .34 & .34 & $3.562 * * *$ & 1.09 & 1.13 & 2.93 & 2.96 & $.62 * * *$ \\
\hline Effort & 4 & .67 & .69 & 3.09 & 3.10 & .58 & .63 & -1.445 & 1.00 & 1.00 & 4.00 & 4.00 & $.51 * * *$ \\
\hline Ability & 4 & .70 & .64 & 2.53 & 2.54 & .79 & .71 & -.176 & 1.00 & 1.00 & 4.00 & 4.00 & $.57 * * *$ \\
\hline Powerful Others & 4 & .70 & .69 & 1.66 & 1.63 & .67 & .64 & .898 & 1.00 & 1.00 & 3.79 & 4.00 & $.61 * * *$ \\
\hline Luck & 4 & .64 & .74 & 1.90 & 1.81 & .70 & .71 & $2.388^{*}$ & 1.00 & 1.00 & 4.00 & 4.00 & $.62 * * *$ \\
\hline Unknown & 4 & .60 & .69 & 1.94 & 1.80 & .68 & .66 & $3.374 * *$ & 1.00 & 1.00 & 4.00 & 4.00 & $.53 * * *$ \\
\hline Total Capacity Beliefs & 23 & .86 & .90 & 3.38 & 3.31 & .42 & .47 & $2.407 *$ & 1.44 & 1.38 & 4.00 & 4.00 & $.66^{* * *}$ \\
\hline Effort & 6 & .58 & .66 & 3.47 & 3.40 & .48 & .52 & $2.677 * *$ & 1.00 & 1.50 & 4.00 & 4.00 & $.50 * * *$ \\
\hline Ability & 6 & .76 & .77 & 3.41 & 3.34 & .50 & .55 & 1.396 & 1.53 & 1.33 & 4.00 & 4.00 & $.61 * * *$ \\
\hline Powerful Others & 5 & .63 & .74 & 3.40 & 3.34 & .57 & .63 & .382 & 1.00 & 1.00 & 4.00 & 4.00 & $.48 * * *$ \\
\hline Luck & 6 & .66 & .76 & 3.23 & 3.16 & .55 & .62 & $2.311 *$ & 1.16 & 1.00 & 4.00 & 4.00 & $.57 * * *$ \\
\hline
\end{tabular}

Note: $N=329 ; * p<.05 . * * p<.01 . * * * p<.001$. 


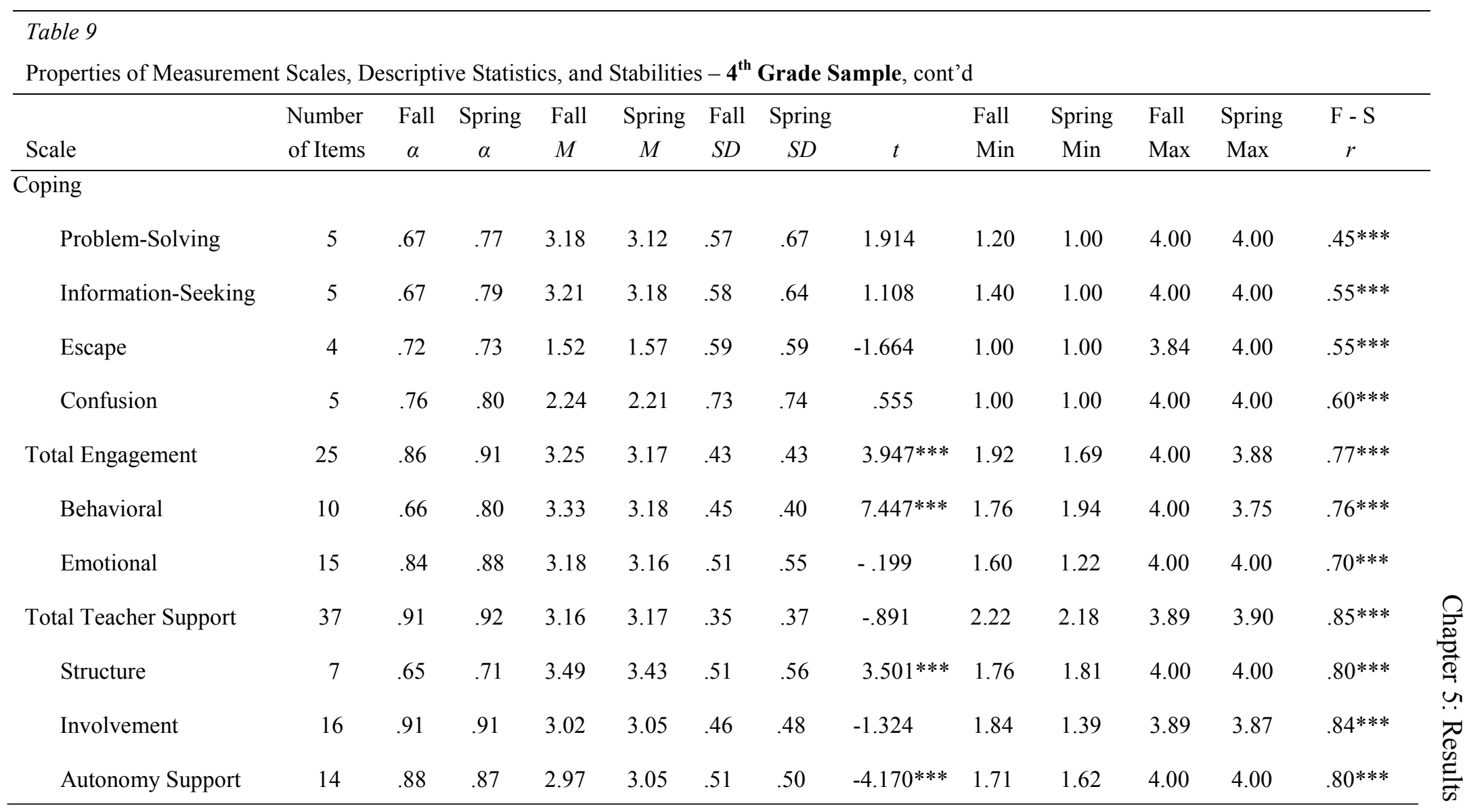

Note: $N=329 ;{ }^{*} p<.05 .{ }^{* *} p<.01 .{ }^{* * *} p<.001$. 


\begin{tabular}{|c|c|c|c|c|c|c|c|c|c|c|c|c|c|}
\hline Table 10 & & & & & & & & & & & & & \\
\hline Properties of Measurement & Scales, De & scriptiy & Statistic & and St & bilities - & $6^{\text {th }}$ Gr: & le Samp & & & & & & \\
\hline Scale & $\begin{array}{l}\text { Number } \\
\text { of Items }\end{array}$ & $\begin{array}{c}\text { Fall } \\
\alpha\end{array}$ & $\begin{array}{c}\text { Spring } \\
\quad \alpha\end{array}$ & $\begin{array}{l}\text { Fall } \\
M\end{array}$ & $\begin{array}{c}\text { Spring } \\
M\end{array}$ & $\begin{array}{l}\text { Fall } \\
S D\end{array}$ & $\begin{array}{l}\text { Spring } \\
S D\end{array}$ & $t$ & $\begin{array}{l}\text { Fall } \\
\text { Min }\end{array}$ & $\begin{array}{l}\text { Spring } \\
\text { Min }\end{array}$ & $\begin{array}{l}\text { Fall } \\
\text { Max }\end{array}$ & $\begin{array}{c}\text { Spring } \\
\text { Max }\end{array}$ & $\begin{array}{c}\mathrm{F}-\mathrm{S} \\
r\end{array}$ \\
\hline Perceived Control & & & & & & & & & & & & & \\
\hline Control Beliefs & 6 & .69 & .71 & 3.38 & 3.29 & .53 & .55 & $3.344^{* *}$ & 1.33 & 1.33 & 4.00 & 4.00 & $.65^{* * *}$ \\
\hline Total Strategy Beliefs & 20 & .79 & .81 & 1.92 & 2.02 & .35 & .38 & $-4.487 * * *$ & 1.12 & 1.00 & 3.13 & 3.04 & $.64 * * *$ \\
\hline Effort & 4 & .67 & .70 & 3.24 & 3.07 & .57 & .65 & .510 & 1.00 & 1.00 & 4.00 & 4.00 & $.49 * * *$ \\
\hline Ability & 4 & .68 & .70 & 2.56 & 2.52 & .67 & .70 & 1.281 & 1.00 & 1.00 & 4.00 & 4.00 & $.53 * * *$ \\
\hline Powerful Others & 4 & .71 & .72 & 1.79 & 1.93 & .65 & .66 & $-4.506 * * *$ & 1.00 & 1.00 & 4.00 & 3.76 & $.58 * * *$ \\
\hline Luck & 4 & .75 & .74 & 1.76 & 1.86 & .65 & .67 & $-3.464 * *$ & 1.00 & 1.00 & 4.00 & 4.00 & $.63 * * *$ \\
\hline Unknown & 4 & .68 & .72 & 1.86 & 1.91 & .65 & .68 & $-2.017 *$ & 1.00 & 1.00 & 4.00 & 3.76 & $.63 * * *$ \\
\hline Total Capacity Beliefs & 23 & .91 & .90 & 3.14 & 3.04 & .48 & .47 & $4.895 * * *$ & 1.44 & 1.93 & 4.00 & 4.00 & $.77 * * *$ \\
\hline Effort & 6 & .74 & .73 & 3.22 & 3.10 & .57 & .57 & $4.474 * * *$ & 1.00 & 1.69 & 4.00 & 4.00 & $.69 * * *$ \\
\hline Ability & 6 & .81 & .78 & 3.21 & 3.08 & .57 & .58 & $3.396^{* *}$ & 1.33 & 1.60 & 4.00 & 4.00 & $.72 * * *$ \\
\hline Powerful Others & 5 & .75 & .73 & 3.21 & 3.10 & .63 & .61 & $2.693 * *$ & 1.16 & 1.00 & 4.00 & 4.00 & $.64 * * *$ \\
\hline Luck & 6 & .66 & .65 & 2.94 & 2.86 & .57 & .55 & $2.738^{* *}$ & 1.33 & 1.49 & 4.00 & 4.00 & $.57 * * *$ \\
\hline
\end{tabular}

Note: $N=336 ;{ }^{*} p<.05 .{ }^{* *} p<.01 .{ }^{* * *} p<.001$. 


\begin{tabular}{|c|c|c|c|c|c|c|c|c|c|c|c|c|c|}
\hline \multicolumn{14}{|l|}{ Table 10} \\
\hline Scale & $\begin{array}{l}\text { Number } \\
\text { of Items }\end{array}$ & $\begin{array}{c}\text { Fall } \\
\alpha\end{array}$ & $\begin{array}{c}\text { Spring } \\
\alpha\end{array}$ & $\begin{array}{l}\text { Fall } \\
M\end{array}$ & $\begin{array}{c}\text { Spring } \\
M\end{array}$ & $\begin{array}{l}\text { Fall } \\
S D\end{array}$ & $\begin{array}{c}\text { Spring } \\
S D\end{array}$ & $t$ & $\begin{array}{l}\text { Fall } \\
\text { Min }\end{array}$ & $\begin{array}{l}\text { Spring } \\
\text { Min }\end{array}$ & $\begin{array}{l}\text { Fall } \\
\text { Max }\end{array}$ & $\begin{array}{c}\text { Spring } \\
\text { Max }\end{array}$ & $\begin{array}{c}\mathrm{F}-\mathrm{S} \\
r \\
\end{array}$ \\
\hline \multicolumn{14}{|l|}{ Coping } \\
\hline Problem-Solving & 5 & .66 & .73 & 3.09 & 2.92 & .53 & .57 & $5.177 * * *$ & 1.00 & 1.39 & 4.00 & 4.00 & $.45^{* * *}$ \\
\hline Information-Seeking & 5 & .77 & .77 & 3.09 & 3.00 & .59 & .58 & $2.185^{* *}$ & 1.20 & 1.33 & 4.00 & 4.00 & $.62 * * *$ \\
\hline Escape & 4 & .79 & .68 & 1.77 & 1.91 & .66 & .61 & $-4.757 * * *$ & 1.00 & 1.00 & 4.00 & 3.56 & $.62 * * *$ \\
\hline Confusion & 5 & .75 & .72 & 2.29 & 2.31 & .66 & .64 & -.692 & 1.00 & 1.00 & 4.00 & 4.00 & $.59 * * *$ \\
\hline Total Engagement & 25 & .90 & .91 & 3.10 & 2.93 & .45 & .41 & $9.506 * * *$ & 1.85 & 1.63 & 4.00 & 3.82 & $.79 * * *$ \\
\hline Behavioral & 10 & .80 & .82 & 3.19 & 2.98 & .50 & .41 & $11.859^{* * *}$ & 1.86 & 1.58 & 4.00 & 3.75 & $.80 * * *$ \\
\hline Emotional & 15 & .86 & .87 & 2.98 & 2.87 & .50 & .50 & $4.936^{* * *}$ & 1.31 & 1.33 & 4.00 & 4.00 & $.72 * * *$ \\
\hline Total Teacher Support & 37 & .92 & .93 & 3.02 & 3.12 & .36 & .42 & $-5.273 * * *$ & 1.82 & 2.05 & 3.76 & 3.96 & $.73 * * *$ \\
\hline Structure & 7 & .64 & .78 & 3.21 & 3.37 & .47 & .52 & $-6.214 * * *$ & 1.33 & 1.77 & 4.00 & 4.00 & $.59 * * *$ \\
\hline Involvement & 16 & .88 & .89 & 2.99 & 3.07 & .43 & .48 & $-3.516^{* * *}$ & 2.01 & 1.71 & 3.93 & 4.00 & $.74 * * *$ \\
\hline Autonomy Support & 14 & .90 & .90 & 2.87 & 2.92 & .49 & .55 & -1.895 & 1.42 & 1.49 & 3.80 & 4.00 & $.75 * * *$ \\
\hline
\end{tabular}


Table 11

Intra-construct Correlations: Components of Perceived Control - Full Sample

\begin{tabular}{|c|c|c|c|c|c|c|c|c|c|c|}
\hline & \multicolumn{6}{|c|}{ Strategy Beliefs } & \multicolumn{4}{|c|}{ Capacity Beliefs } \\
\hline & $\begin{array}{l}\text { Control } \\
\text { Beliefs }\end{array}$ & Effort & Ability & $\begin{array}{l}\text { Powerful } \\
\text { Others }\end{array}$ & Luck & Unknown & Effort & Ability & $\begin{array}{c}\text { Powerful } \\
\text { Others }\end{array}$ & Luck \\
\hline Control Beliefs & - & -.01 & $-.12 * *$ & $-.48 * * *$ & $-.51 * * *$ & $-.60 * * *$ & $.67 * * *$ & $.52 * * *$ & $.48 * * *$ & $.59^{* * *}$ \\
\hline \multicolumn{11}{|l|}{ Strategy } \\
\hline Effort & $.15^{* * *}$ & - & $.23 * * *$ & .06 & .06 & $.11^{* *}$ & -.07 & $-.08^{*}$ & -.07 & $-.13 * *$ \\
\hline Ability & $-.24 * * *$ & $.21 * * *$ & - & $.26^{* * *}$ & $.31 * * *$ & $.17 * * *$ & $-.15^{* * *}$ & $-.21 * * *$ & $-.17 * *$ & $-.15^{* * *}$ \\
\hline Powerful Others & $-.48 * * *$ & -.04 & $.30 * * *$ & - & $.49 * * *$ & $.50^{* * *}$ & $-.51 * * *$ & $-.57 * * *$ & $-.45^{* * *}$ & $-.43 * * *$ \\
\hline Luck & $-.50 * * *$ & -.07 & $.31 * * *$ & $.50^{* * *}$ & - & $.52 * * *$ & $-.46^{* * *}$ & $-.46^{* * *}$ & $-.35^{* * *}$ & $-.42 * * *$ \\
\hline Unknown & $-.59 * * *$ & -.03 & $.25^{* * *}$ & $.46^{* * *}$ & $.51 * * *$ & - & $-.59 * * *$ & $-.56 * * *$ & $-.44 * * *$ & $-.55^{* * *}$ \\
\hline \multicolumn{11}{|l|}{ Capacity } \\
\hline Effort & $.67 * * *$ & .03 & $-.21 * * *$ & $-.44 * * *$ & $-.39 * * *$ & $-.50^{* * *}$ & - & $.66^{* * *}$ & $.49 * * *$ & $.66^{* * *}$ \\
\hline Ability & $.67 * * *$ & .06 & $-.26 * * *$ & $-.47 * * *$ & $-.45 * * *$ & $-.50^{* * *}$ & $.63 * * *$ & - & $.76 * * *$ & $.67 * * *$ \\
\hline Powerful Others & $.50^{* * *}$ & $.08^{*}$ & $-.20^{* * *}$ & $-.40^{* * *}$ & $-.37^{* * *}$ & $-.40 * * *$ & $.48^{* * *}$ & $.72 * * *$ & - & $.50 * * *$ \\
\hline Luck & $.59 * * *$ & .03 & $-.21 * * *$ & $-.36 * * *$ & $-.33 * * *$ & $-.43 * * *$ & $.54 * * *$ & $.65 * * *$ & $.45 * * *$ & - \\
\hline
\end{tabular}

Note: $N=665$; correlations for fall are below the diagonal; correlations for spring are above the diagonal; ${ }^{*} p<.05 .{ }^{* *} p<.01 .{ }^{* * *} p<.001$. 
Table 12

Intra-construct Correlations: Components of Perceived Control $-4^{\text {th }}$ Grade

\begin{tabular}{|c|c|c|c|c|c|c|c|c|c|c|}
\hline & \multicolumn{6}{|c|}{ Strategy Beliefs } & \multicolumn{4}{|c|}{ Capacity Beliefs } \\
\hline & $\begin{array}{l}\text { Control } \\
\text { Beliefs }\end{array}$ & Effort & Ability & $\begin{array}{l}\text { Powerful } \\
\text { Others }\end{array}$ & Luck & Unknown & Effort & Ability & $\begin{array}{c}\text { Powerful } \\
\text { Others }\end{array}$ & Luck \\
\hline Control Beliefs & - & -.01 & -.09 & $-.39 * * *$ & $-.46^{* * *}$ & $-.57^{* * *}$ & $.65^{* * *}$ & $.55^{* * *}$ & $.40^{* * *}$ & $.59^{* * *}$ \\
\hline \multicolumn{11}{|l|}{ Strategy } \\
\hline Effort & $.11^{*}$ & - & $.30^{* * *}$ & .03 & $.12^{*}$ & $.18^{* *}$ & -.04 & -.09 & -.06 & $-.11^{*}$ \\
\hline Ability & $-.24^{* * *}$ & $.31^{* * *}$ & - & $.26^{* *}$ & $.31^{* * *}$ & $.17^{* *}$ & $-.15^{* *}$ & $-.22 *$ & $-.20 * *$ & $-.17^{* *}$ \\
\hline Powerful Others & $-.42 * * *$ & -.003 & $.30^{* * *}$ & - & $.45^{* * *}$ & $.42 * * *$ & $-.45^{* * *}$ & $-.49 * * *$ & $-.37 * * *$ & $-.36^{* * *}$ \\
\hline Luck & $-.45^{* * *}$ & .07 & $.36^{* * *}$ & $.52 * * *$ & - & $.49 * * *$ & $-.44 * * *$ & $-.35 * * *$ & $-.28 * * *$ & $-.41 * * *$ \\
\hline Unknown & $-.55^{* * *}$ & .07 & $.24 * * *$ & $.47 * * *$ & $.48 * * *$ & - & $-.57 * * *$ & $-.47 * * *$ & $-.36^{* * * *}$ & $-.58 * * *$ \\
\hline
\end{tabular}

\begin{tabular}{|c|c|c|c|c|c|c|c|c|c|c|}
\hline \multicolumn{11}{|l|}{ Capacity } \\
\hline Effort & $.58 * * *$ & -.01 & $-.16^{* *}$ & $-.37 * * *$ & $-.33 * * *$ & $-.47 * * *$ & - & $.57 * * *$ & $.41 * * *$ & $.65^{* * *}$ \\
\hline Ability & $.63 * * *$ & .01 & $-.24 * * *$ & $-.46^{* * *}$ & $-.41 * * *$ & $-.41 * * *$ & $.54 * * *$ & - & $.72 * * *$ & $.65^{* * *}$ \\
\hline Powerful Others & $.44 * * *$ & .03 & $-.17^{* *}$ & $-.36^{* * *}$ & $-.31 * * *$ & $-.34 * * *$ & $.35^{* * *}$ & $.68 * * *$ & - & $.46^{* * *}$ \\
\hline Luck & $.55^{* * *}$ & .01 & $-.23 * * *$ & $-.32 * * *$ & $-.29 * * *$ & $-.41 * * *$ & $.47^{* * * *}$ & $.62 * * *$ & $.43^{* * *}$ & - \\
\hline
\end{tabular}


Table 13

Intra-construct Correlations: Components of Perceived Control $-6^{\text {th }}$ Grade

\begin{tabular}{|c|c|c|c|c|c|c|c|c|c|c|}
\hline & \multicolumn{6}{|c|}{ Strategy Beliefs } & \multicolumn{4}{|c|}{ Capacity Beliefs } \\
\hline & $\begin{array}{r}\text { Control } \\
\text { Beliefs }\end{array}$ & Effort & Ability & $\begin{array}{l}\text { Powerful } \\
\text { Others }\end{array}$ & Luck & Unknown & Effort & Ability & $\begin{array}{c}\text { Powerful } \\
\text { Others }\end{array}$ & Luck \\
\hline Control Beliefs & - & .03 & $-.16^{* *}$ & $-.52 * * *$ & $-.57^{* * *}$ & $-.62 * * *$ & $.67^{* * *}$ & $.65^{* * *}$ & $.51^{* * *}$ & $.57^{* * *}$ \\
\hline \multicolumn{11}{|l|}{ Strategy } \\
\hline Effort & $.20 * * *$ & - & $.37 * * *$ & .04 & -.01 & .01 & -.03 & -.01 & -.02 & -.07 \\
\hline Ability & $-.25^{* * *}$ & .09 & - & $.29 * * *$ & $.31 * * *$ & $.18^{* *}$ & $-.21 * *$ & $-.22 * *$ & $-.15^{* *}$ & $-.14^{* *}$ \\
\hline Powerful Others & $-.53^{* * *}$ & -.10 & $.30^{* * *}$ & - & $.54^{* * *}$ & $.56^{* * *}$ & $-.51 * * *$ & $-.59 * * *$ & $-.48^{* * *}$ & $-.44 * * *$ \\
\hline Luck & $-.57^{* * *}$ & $-.19 * *$ & $.26 * * *$ & $.50^{* * *}$ & - & $.55^{* * *}$ & $-.50^{* * *}$ & $-.57 * * *$ & $-.47 * * *$ & $-.38 * * *$ \\
\hline Unknown & $-.65^{* * *}$ & $-.13^{*}$ & $.26 * * *$ & $.47 * * *$ & $.54 * * *$ & - & $-.62 * * *$ & $-.63 * * *$ & $-.52 * * *$ & $-.52 * * *$ \\
\hline
\end{tabular}

\begin{tabular}{|c|c|c|c|c|c|c|c|c|c|c|}
\hline \multicolumn{11}{|l|}{ Capacity } \\
\hline Effort & $.75 * * *$ & $.11^{*}$ & $-.27 * * *$ & $-.49 * * *$ & $-.51 * * *$ & $-.60 * * *$ & - & $.69 * * *$ & $.52 * * *$ & $.63^{* * *}$ \\
\hline Ability & $.70 * * *$ & $.16^{* *}$ & $-.29 * * *$ & $-.47 * * *$ & $-.55^{* * *}$ & $-.62^{* * *}$ & $.66^{* * *}$ & - & $.77 * * *$ & $.65^{* * * *}$ \\
\hline Powerful Others & $.54 * * *$ & $.16^{* *}$ & $-.24 * * *$ & $-.42 * * *$ & $-.48 * * *$ & $-.48^{* * *}$ & $.54 * * *$ & $.74 * * *$ & - & $.49^{* * *}$ \\
\hline Luck & $.63 * * *$ & $.12 *$ & $-.21^{* * *}$ & $-.36 * * *$ & $-.46 * * *$ & $-.52 * * *$ & $.55^{* * *}$ & $.64 * * *$ & $.42 * * *$ & - \\
\hline
\end{tabular}


Table 14

Intra-construct Correlations: Components of Engagement, Coping, and Teacher Support - Full Sample

\begin{tabular}{ccccccccccc}
\hline Engagement & \multicolumn{4}{c}{ Coping } & \multicolumn{3}{c}{ Teacher Support } \\
\hline Behavioral & $\begin{array}{c}\text { Emotional } \\
\text { Eng }\end{array}$ & $\begin{array}{c}\text { Probl- } \\
\text { Eng }\end{array}$ & $\begin{array}{c}\text { Solv } \\
\text { See- }\end{array}$ & Seek & Escp & Conf & Structure & Involvement & Autonomy \\
Support
\end{tabular}

Engagement

$\begin{array}{lcc}\text { Behavioral Engagement } & - & .72 * * * \\ \text { Emotional Engagement } & .63 * * * & -\end{array}$

\begin{tabular}{|c|c|c|c|c|}
\hline \multicolumn{5}{|l|}{ Coping } \\
\hline Problem-Solving & - & $.65 * * *$ & $-.35 * * *$ & .01 \\
\hline Information-Seeking & $.56 * * *$ & - & $-.39 * * *$ & $-.15 * * *$ \\
\hline Escape & $-.28 * * *$ & $-.36 * * *$ & - & $.45 * * *$ \\
\hline Confusion & $.09^{*}$ & $-.09 *$ & $.45 * * *$ & - \\
\hline
\end{tabular}

Teacher Support

Structure

Involvement

Autonomy Support

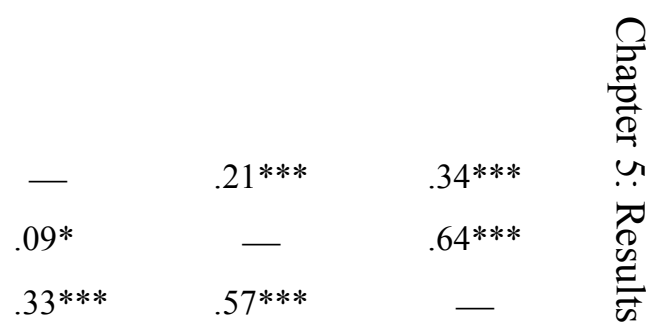

Note: $N=665$; correlations for fall are below the diagonal; correlations for spring are above the diagonal; ${ }^{*} p<.05 .{ }^{* *} p<.01 .{ }^{* * *} p<.001$. 
Table 15

Intra-construct Correlations: Components of Engagement, Coping, and Teacher Support $-\mathbf{4}^{\text {th }}$ Grade

\begin{tabular}{|c|c|c|c|c|c|c|c|c|c|}
\hline & \multicolumn{2}{|c|}{ Engagement } & \multicolumn{4}{|c|}{ Coping } & \multicolumn{3}{|c|}{ Teacher Support } \\
\hline & $\begin{array}{c}\text { Behavioral } \\
\text { Eng }\end{array}$ & $\begin{array}{l}\text { Emotional } \\
\text { Eng }\end{array}$ & $\begin{array}{l}\text { Probl- } \\
\text { Solv }\end{array}$ & $\begin{array}{l}\text { Info- } \\
\text { Seek }\end{array}$ & Escp & Conf & Structure & Involvement & $\begin{array}{l}\text { Autonomy } \\
\text { Support }\end{array}$ \\
\hline \multicolumn{10}{|l|}{ Engagement } \\
\hline Behavioral Engagement & - & $.71 * * *$ & & & & & & & \\
\hline Emotional Engagement & $.60 * * *$ & - & & & & & & & \\
\hline \multicolumn{10}{|l|}{ Coping } \\
\hline Problem-Solving & & & - & $.65^{* * *}$ & $-.28 * * *$ & .08 & & & \\
\hline Information-Seeking & & & $.54 * * *$ & - & $-.32 * * *$ & $-.15 * *$ & & & \\
\hline Escape & & & $-.18 * *$ & $-.20 * * *$ & - & & & & \\
\hline Confusion & & & $.16^{* *}$ & -.03 & $.48 * * *$ & - & & & \\
\hline Structure & & & & & & & - & .02 & $.24 * * *$ \\
\hline Involvement & & & & & & & -.07 & - & $.65 * * *$ \\
\hline Autonomy Support & & & & & & & $.32 * * *$ & $.54 * * *$ & - \\
\hline
\end{tabular}




\section{Table 16}

Intra-construct Correlations: Components of Engagement, Coping, and Teacher Support $-\mathbf{6}^{\text {th }}$ Grade

\begin{tabular}{|c|c|c|c|c|c|c|c|c|c|}
\hline & \multicolumn{2}{|c|}{ Engagement } & \multicolumn{4}{|c|}{ Coping } & \multicolumn{3}{|c|}{ Teacher Support } \\
\hline & $\begin{array}{c}\text { Behavioral } \\
\text { Eng }\end{array}$ & $\begin{array}{l}\text { Emotional } \\
\text { Eng }\end{array}$ & $\begin{array}{l}\text { Probl- } \\
\text { Solv }\end{array}$ & $\begin{array}{l}\text { Info- } \\
\text { Seek }\end{array}$ & Escp & Conf & Structure & Involvement & $\begin{array}{l}\text { Autonomy } \\
\text { Support }\end{array}$ \\
\hline \multicolumn{10}{|l|}{ Engagement } \\
\hline Behavioral Engagement & - & $.70 * * *$ & & & & & & & \\
\hline Emotional Engagement & $.64 * * *$ & - & & & & & & & \\
\hline \multicolumn{10}{|l|}{ Coping } \\
\hline Problem-Solving & & & - & $.64 * * *$ & $-.38 * * *$ & .05 & & & \\
\hline Information-Seeking & & & $.57 * * *$ & - & $-.44 * * *$ & $-.13 *$ & & & \\
\hline Escape & & & $-.37 * * *$ & $-.47 * * *$ & - & $.46^{* * *}$ & & & \\
\hline $\begin{array}{c}\text { Confusion } \\
\text { Teacher Support }\end{array}$ & & & .002 & $-.14^{*}$ & $.42 * * *$ & - & & & \\
\hline Structure & & & & & & & - & $.42 * * *$ & $.42 * * *$ \\
\hline Involvement & & & & & & & $.26 * * *$ & - & $.65 * * *$ \\
\hline Autonomy Support & & & & & & & $.30 * * *$ & $.60 * * *$ & - \\
\hline
\end{tabular}




\begin{tabular}{|c|c|c|c|c|c|c|c|c|c|c|}
\hline \multicolumn{11}{|c|}{ Table 17} \\
\hline \multicolumn{11}{|c|}{ Inter-Construct Correlations - Full Sample } \\
\hline & \multicolumn{3}{|c|}{ Control } & \multicolumn{4}{|c|}{ Coping } & \multirow[b]{2}{*}{ Total Eng } & \multirow[b]{2}{*}{$\begin{array}{l}\text { Total Tchr } \\
\text { Sup }\end{array}$} & \multirow[b]{2}{*}{ Achvmnt } \\
\hline & Tot Strat & Tot Cap & Conmax & $\begin{array}{l}\text { Problem- } \\
\text { Solving }\end{array}$ & $\begin{array}{c}\text { Info- } \\
\text { Seeking }\end{array}$ & Escape & Conf & & & \\
\hline \multicolumn{11}{|l|}{ Control } \\
\hline Total Strategy Beliefs & - & $-.51 * * *$ & $-.63 * * *$ & $-.33 * * *$ & $-.28 * * *$ & $.53 * * *$ & $.20 * * *$ & $-.42 * * *$ & $-.32 * * *$ & $-.38 * * *$ \\
\hline Total Capacity Beliefs & $-.50 * * *$ & - & $.96^{* * *}$ & $.42 * * *$ & $.56^{* * *}$ & $-.71 * * *$ & $-.44 * * *$ & $.76^{* * *}$ & $.33^{* * *}$ & $.33^{* * *}$ \\
\hline Conmax & $-.62 * * *$ & $.96^{* * *}$ & - & $.41^{* * *}$ & $.47 * * *$ & $-.75^{* * *}$ & $-.46^{* * *}$ & $.77 * * *$ & $.36^{* * *}$ & $.37 * * *$ \\
\hline \multicolumn{11}{|l|}{ Coping } \\
\hline Problem-Solving & $-.26 * * *$ & $.37 * * *$ & $.35 * * *$ & - & & & & $.47 * * *$ & $.14 * * *$ & $.21 * * *$ \\
\hline Information-Seeking & $-.27 * * *$ & $.46^{* * *}$ & $.45^{* * *}$ & & - & & & $.54 * * *$ & $.11^{* *}$ & $.12 * *$ \\
\hline Escape & $.48^{* * *}$ & $-.65 * * *$ & $-.69 * * *$ & & & - & & $-.72 * * *$ & $-.27 * * *$ & $-.41 * * *$ \\
\hline Confusion & $.24 * * *$ & $-.40^{* * *}$ & $-.46^{* * *}$ & & & & - & $-.47^{* * *}$ & $-.08^{*}$ & -.07 \\
\hline \multicolumn{11}{|l|}{ Engagement } \\
\hline Total Engagement & $-.44 * * *$ & $.72 * * *$ & $.75^{* * *}$ & $.36^{* * *}$ & $.47 * * *$ & $-.67^{* * *}$ & $-.48 * * *$ & - & $.27 * * *$ & $.19 * * *$ \\
\hline \multicolumn{11}{|l|}{ Teacher Support } \\
\hline Total Teacher Support & $-.29 * * *$ & $.34 * * *$ & $.35^{* * *}$ & $.10^{*}$ & $.14^{* *}$ & $-.26 * * *$ & $-.10^{*}$ & $.29 * * *$ & - & $.20 * * *$ \\
\hline Achievement & $-.37 * * *$ & $.25 * * *$ & $.31 * * *$ & $.10^{* *}$ & $.08^{*}$ & $-.34 * * *$ & $-.13 * *$ & $.32 * * *$ & $.24 * * *$ & - \\
\hline
\end{tabular}




\begin{tabular}{|c|c|c|c|c|c|c|c|c|c|c|}
\hline \multicolumn{11}{|c|}{$\begin{array}{l}\text { Table } 18 \\
\text { Inter-Construct Correlations }-4^{\text {th }} \text { Grade Sample }\end{array}$} \\
\hline & \multicolumn{3}{|c|}{ Control } & \multicolumn{4}{|c|}{ Coping } & \multirow[b]{2}{*}{ Total Eng } & \multirow[b]{2}{*}{$\begin{array}{c}\text { Total } \\
\text { Tchr Sup }\end{array}$} & \multirow[b]{2}{*}{ Achvmnt } \\
\hline & Tot Strat & Tot Cap & Conmax & $\begin{array}{c}\text { Problem- } \\
\text { Solving }\end{array}$ & $\begin{array}{c}\text { Info- } \\
\text { Seeking }\end{array}$ & Escape & Conf & & & \\
\hline \multicolumn{11}{|l|}{ Control } \\
\hline Total Strategy Beliefs & - & $-.54 * * *$ & $-.58 * * *$ & $-.23 * * *$ & $-.18 * *$ & $.49 * * *$ & $.22 * * *$ & $-.37 * * *$ & $-.25^{* * *}$ & $-.39 * *$ \\
\hline Total Capacity Beliefs & $-.45^{* * *}$ & - & $.95 * * *$ & $.38 * * *$ & $.48^{* * *}$ & $-.67 * * *$ & $-.48 * * *$ & $.72 * * *$ & $.26 * * *$ & $.40 * * *$ \\
\hline Conmax & $-.57 * * *$ & $.94 * * *$ & - & $.35^{* * *}$ & $.43 * * *$ & $-.71 * * *$ & $-.50^{* * *}$ & $.73 * * *$ & $.30 * * *$ & $.44 * * *$ \\
\hline \multicolumn{11}{|l|}{ Coping } \\
\hline Problem-Solving & $-.19 * *$ & $.28 * * *$ & $.23 * * *$ & - & & & & $.43 * * *$ & .05 & $.34 * * *$ \\
\hline Information-Seeking & $-.16^{* *}$ & $.38 * * *$ & $.35^{* * *}$ & & - & & & $.53 * * *$ & .07 & $.29 * * *$ \\
\hline Escape & $.47 * * *$ & $-.61 * * *$ & $-.66^{* * *}$ & & & - & & $-.68 * * *$ & $-.25^{* * *}$ & $-.56 * * *$ \\
\hline Confusion & $.25 * * *$ & $-.40 * * *$ & $-.48 * * *$ & & & & - & $-.50 * * *$ & -.08 & -.01 \\
\hline \multicolumn{11}{|l|}{ Engagement } \\
\hline Total Engagement & $-.39 * * *$ & $.66^{* * *}$ & $.69 * * *$ & $.23 * * *$ & $.32 * * *$ & $-.60 * * *$ & $-.51 * * *$ & - & $.24 * * *$ & $.44 * * *$ \\
\hline \multicolumn{11}{|l|}{ Teacher Support } \\
\hline Total Teacher Support & $-.22 * * *$ & $.25 * * *$ & $.28 * * *$ & .07 & .07 & $-.21 * * *$ & -.07 & $.24 * * *$ & - & $.33 * * *$ \\
\hline Achievement & $-.32 * * *$ & $.27 * * *$ & $.32 * * *$ & .10 & .08 & $-.34 * * *$ & $.13 *$ & $.41 * * *$ & $.25 * * *$ & - \\
\hline
\end{tabular}




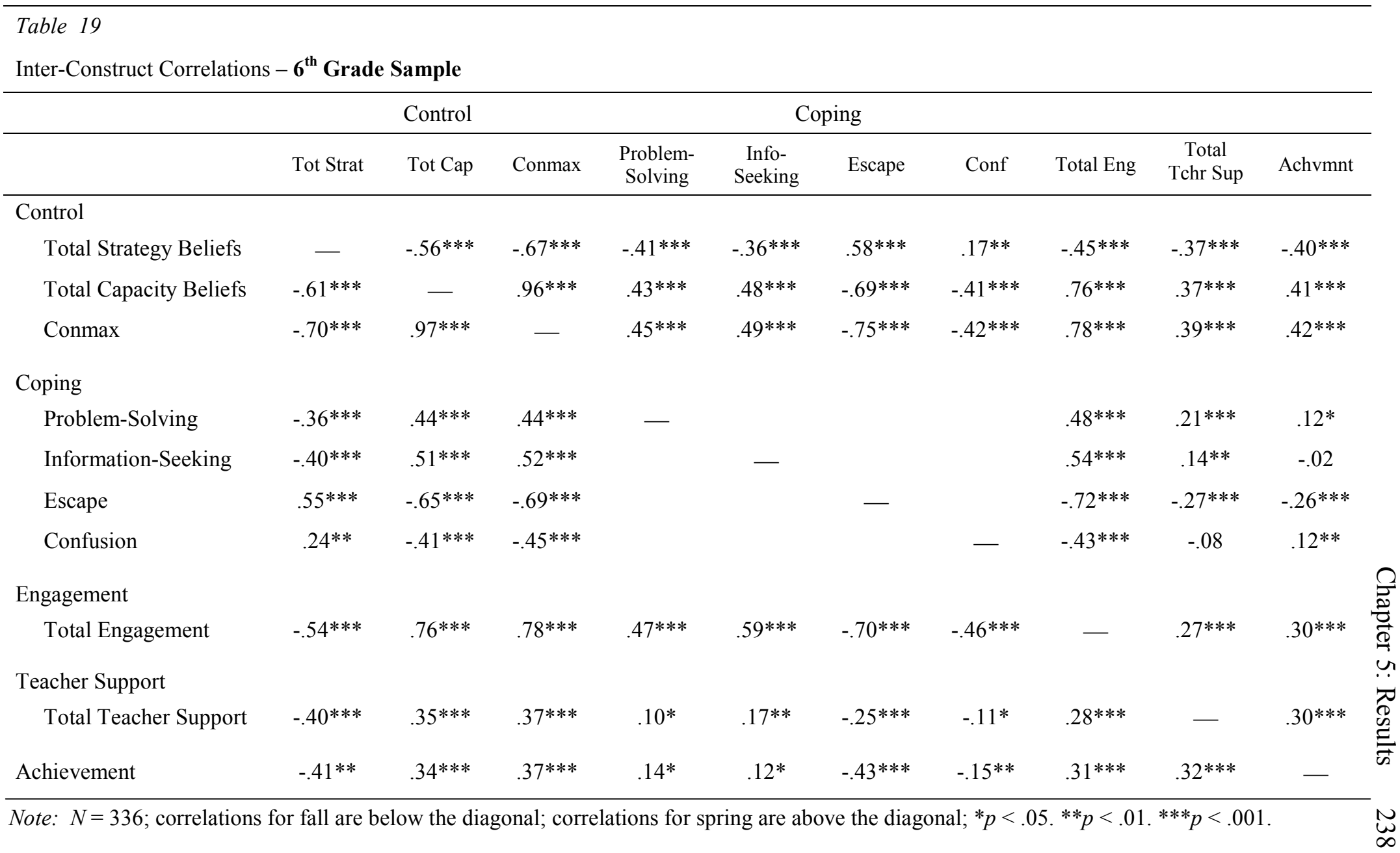


Table 20

Age Differences in Construct Means by Time of Measurement

\begin{tabular}{|c|c|c|c|c|c|}
\hline & \multicolumn{2}{|c|}{ Fall } & \multicolumn{2}{|c|}{ Spring } & \\
\hline & $t$ & Mean Difference & $t$ & Mean Difference & \\
\hline \multicolumn{6}{|l|}{ Perceived Control } \\
\hline Control Beliefs & 1.956 & 0.08 & $4.875^{* * *}$ & .21 & \\
\hline Total Strategy Beliefs & $3.330^{* *}$ & 0.09 & $-2.279^{*}$ & -.07 & \\
\hline Effort & $-5.147 * * *$ & -0.34 & $-3.174 * *$ & -.23 & \\
\hline Ability & -.575 & -0.03 & .527 & .03 & \\
\hline Powerful Others & $-2.528^{*}$ & -0.13 & $-6.480 * * *$ & -.35 & \\
\hline Luck & $2.833^{* *}$ & 0.15 & -1.268 & -.07 & \\
\hline Unknown & 1.593 & 0.08 & $-2.218^{*}$ & -.12 & \\
\hline Total Capacity Beliefs & $6.386^{* * *}$ & 0.23 & $7.343 * * *$ & .28 & \multirow{6}{*}{ 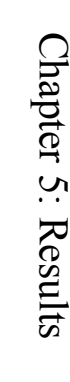 } \\
\hline Effort & $6.038 * * *$ & 0.25 & $6.309 * * *$ & .30 & \\
\hline Ability & $4.488 * * *$ & 0.19 & $5.687 * * *$ & .28 & \\
\hline Powerful Others & $3.913^{* * *}$ & 0.19 & $5.115^{* * *}$ & .28 & \\
\hline Luck & $5.933 * * *$ & 0.28 & $5.635^{* * *}$ & .28 & \\
\hline Conmax & $3.692 * * *$ & 4.80 & $6.092 * * *$ & 8.51 & \\
\hline
\end{tabular}


Table 20

Age Differences in Construct Means by Time of Measurement, cont'd

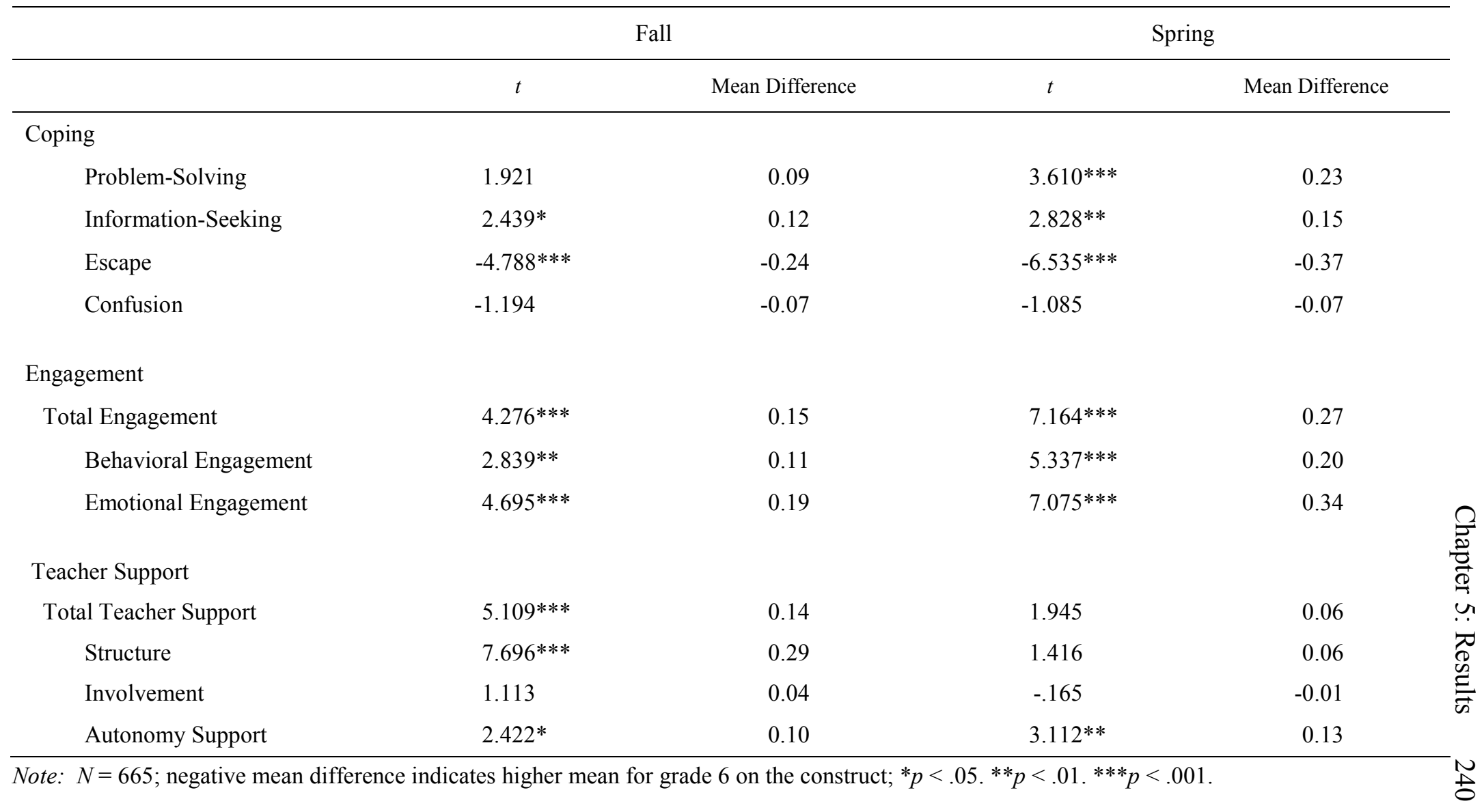


Table 21

Age Differences in Fall-to-Spring Construct Correlations

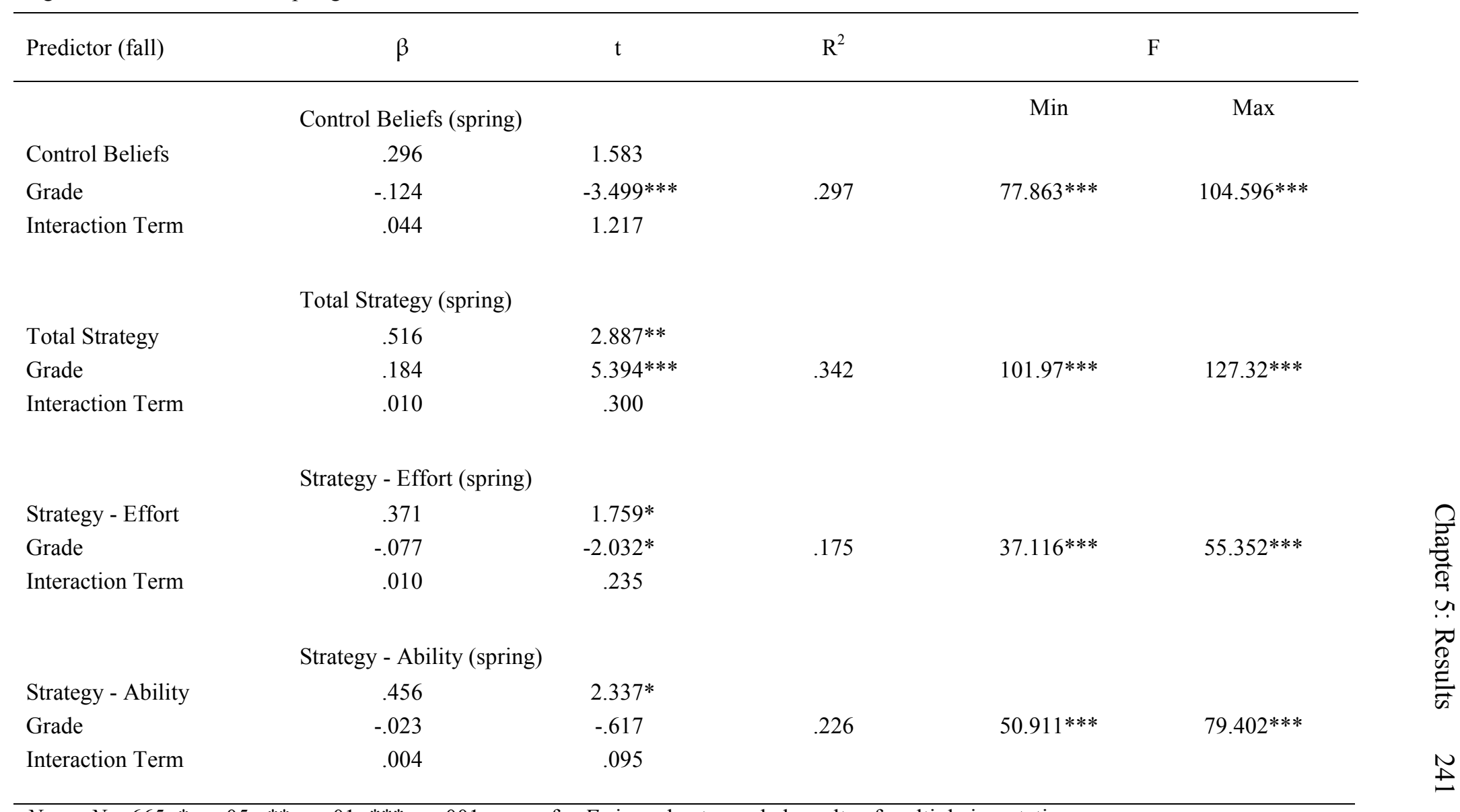

Note: $N=665 ;{ }^{*} p<.05 .{ }^{* *} p<.01 .{ }^{* * *} p<.001$; range for $F$ given due to pooled results of multiple imputation. 
Table 21

Age Differences in Fall-to-Spring Construct Correlations, cont'd

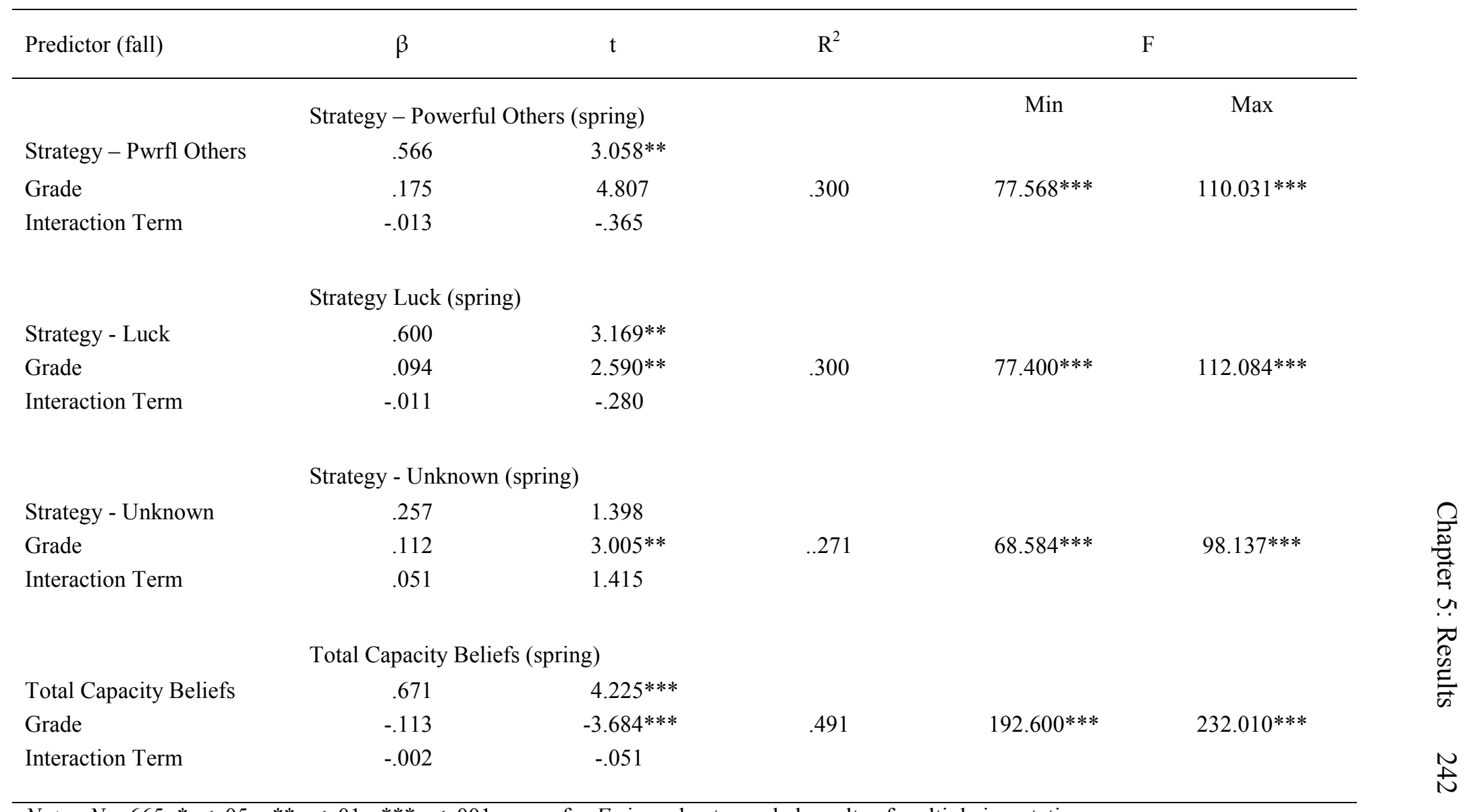

Note: $N=665 ;{ }^{*} p<.05 . \quad{ }^{* *} p<.01 .{ }^{* * *} p<.001$; range for $F$ given due to pooled results of multiple imputation. 
Table 21

Age Differences in Fall-to-Spring Construct Correlations, cont'd

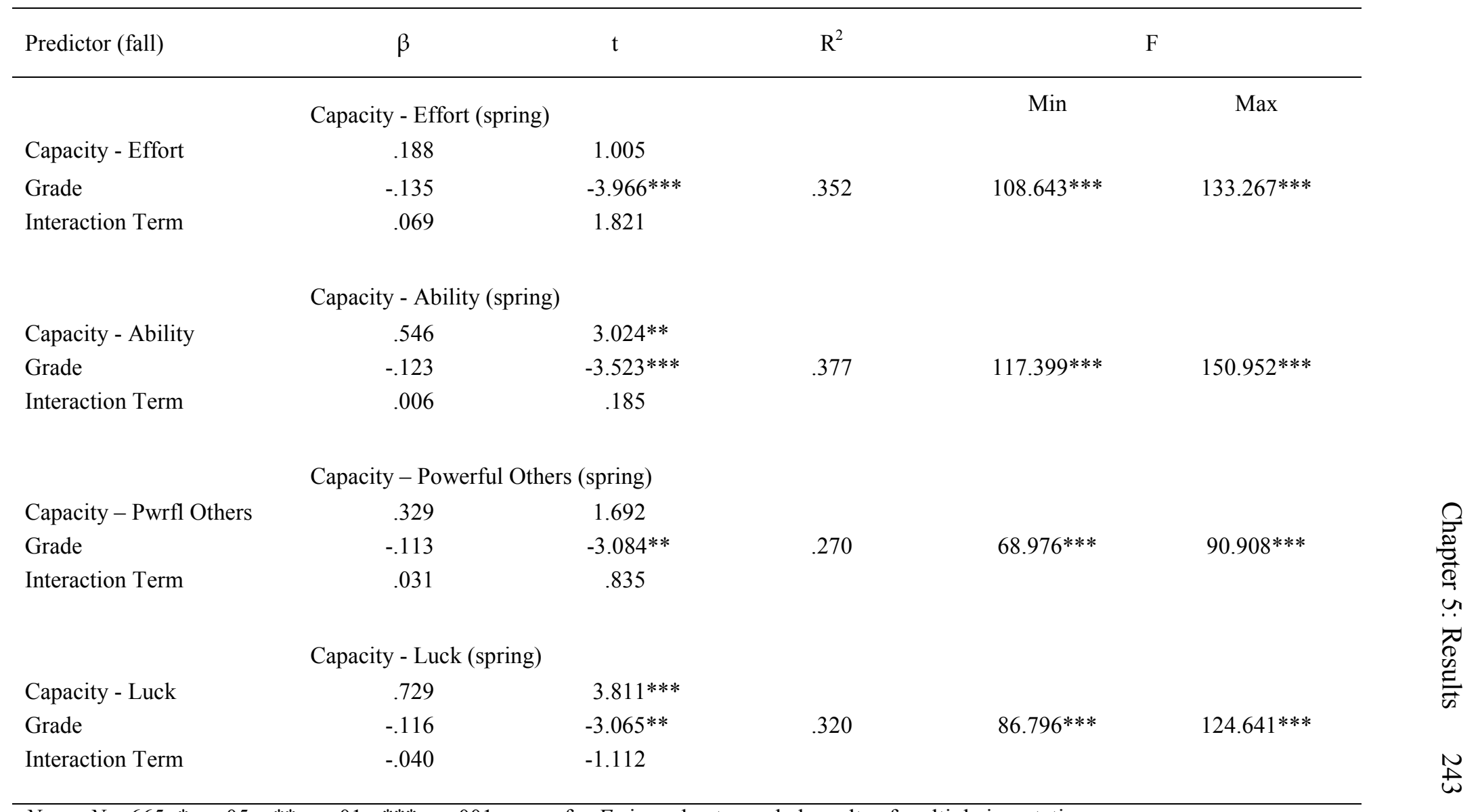

Note: $N=665 ;{ }^{*} p<.05 . \quad{ }^{* *} p<.01 .{ }^{* * *} p<.001$; range for $F$ given due to pooled results of multiple imputation. 
Table 21

Age Differences in Fall-to-Spring Construct Correlations, cont'd

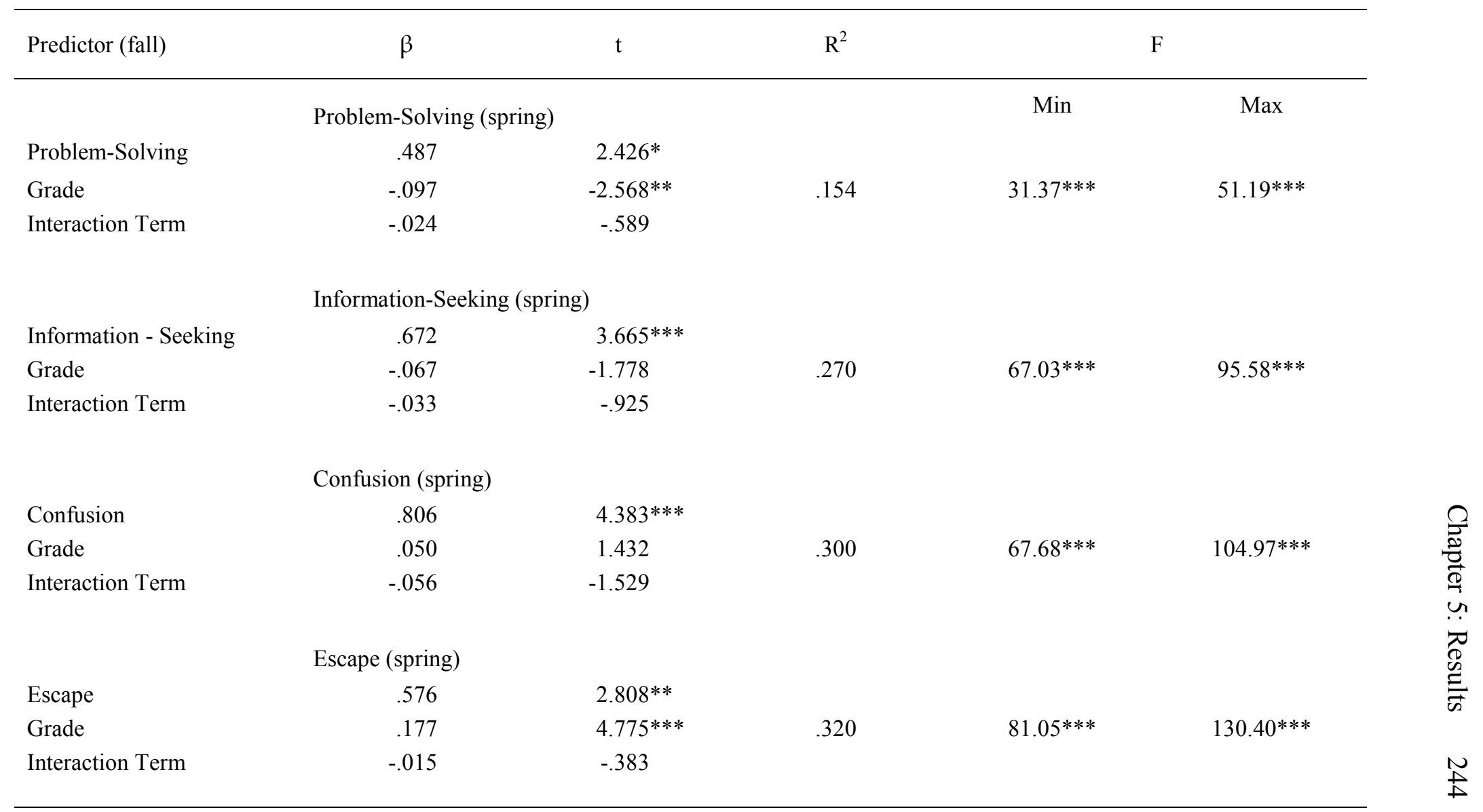

Note: $N=665 ;{ }^{*} p<.05 . \quad{ }^{* *} p<.01 .{ }^{* * *} p<.001$; range for $F$ given due to pooled results of multiple imputation. 
Table 21

Age Differences in Fall-to-Spring Construct Correlations, cont'd

\begin{tabular}{|c|c|c|c|c|c|}
\hline Predictor (fall) & $\beta$ & $\mathrm{t}$ & $\mathrm{R}^{2}$ & & \\
\hline & Engagement (spring) & & & Min & Max \\
\hline Engagement & .918 & $6.431 * * *$ & & & \\
\hline Grade & -.153 & $-5.424 * * *$ & .570 & $266.79 * * *$ & $310.71 * * *$ \\
\hline \multirow[t]{2}{*}{ Interaction Term } & -.041 & -1.491 & & & \\
\hline & \multicolumn{5}{|c|}{ Behavioral Engagement (spring) } \\
\hline Behavioral Engagement & .808 & $5.348 * * *$ & & & \\
\hline Grade & -.151 & $-5.270 * * *$ & .570 & $255.12 * * *$ & $326.40 * * *$ \\
\hline \multirow[t]{2}{*}{ Interaction Term } & -.017 & -.589 & & & \\
\hline & \multicolumn{5}{|c|}{ Emotional Engagement (spring) } \\
\hline Emotional Engagement & .813 & $4.908 * * *$ & & & \\
\hline Grade & -.145 & $-4.638 * * *$ & .463 & $175.54 * * *$ & $204.43 * * *$ \\
\hline \multirow[t]{2}{*}{ Interaction Term } & -.035 & -1.091 & & & \\
\hline & Conmax (spring) & & & & \\
\hline Conmax & .690 & $4.565^{* * *}$ & & & \\
\hline Grade & -.131 & $-4.575 * * *$ & .534 & $233.10 * * *$ & $270.08 * * *$ \\
\hline Interaction Term & .001 & .051 & & & \\
\hline
\end{tabular}

Note: $N=665 ;{ }^{*} p<.05 . \quad{ }^{* *} p<.01 .{ }^{* * *} p<.001$; range for $F$ given due to pooled results of multiple imputation. 
Table 21

Age Differences in Fall-to-Spring Construct Correlations, cont'd

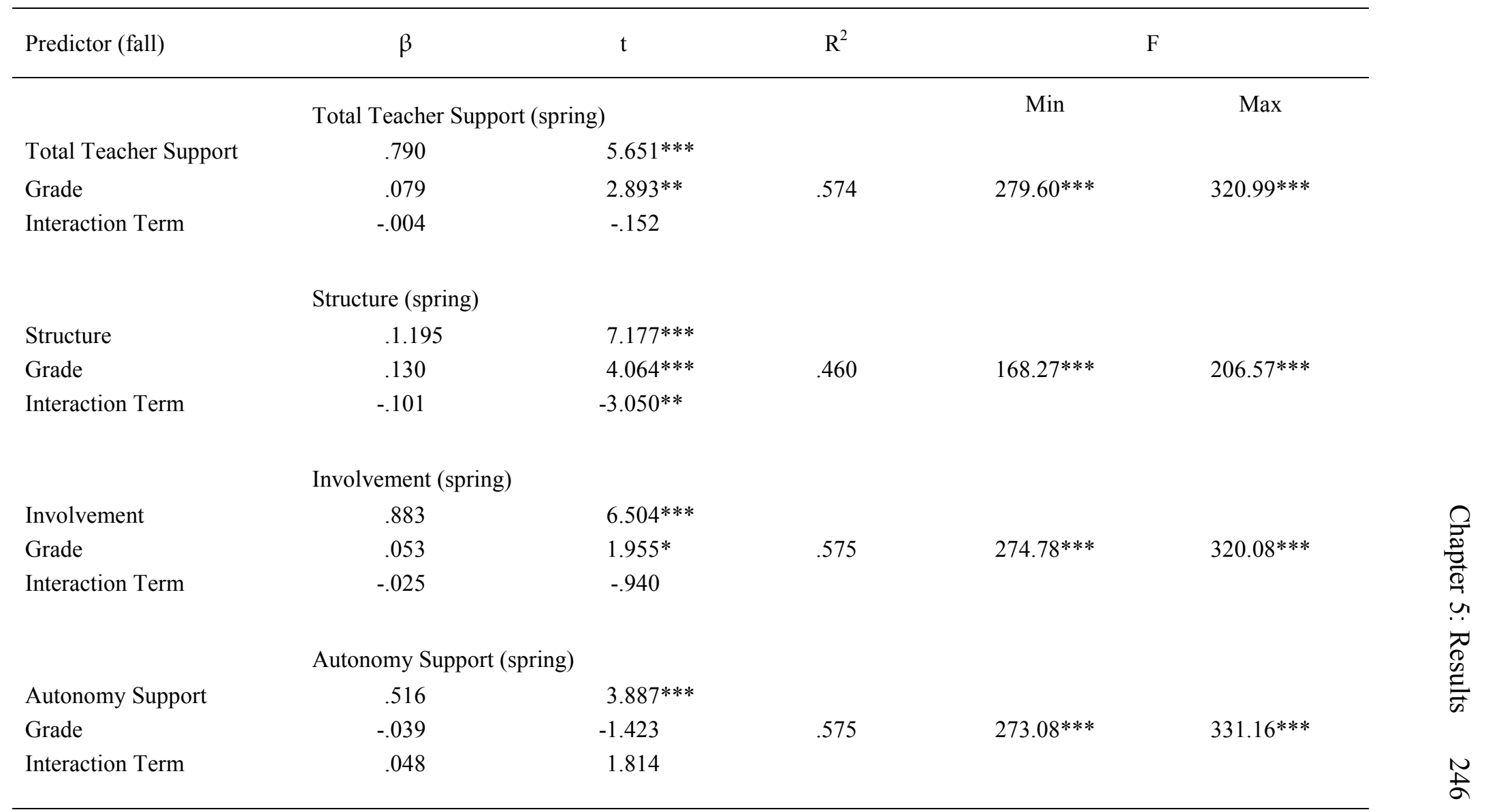

Note: $N=665 ;{ }^{*} p<.05 . \quad{ }^{* *} p<.01 .{ }^{* * *} p<.001 ;$ range for $F$ given due to pooled results of multiple imputation. 
Table 22

Age Differences in Amount of Change from Fall-to-Spring in Construct Means

\begin{tabular}{|c|c|c|c|c|c|}
\hline & $t$ & $\begin{array}{l}\text { Mean Change } \\
\text { Difference }\end{array}$ & & $t$ & $\begin{array}{l}\text { Mean Change } \\
\text { Difference }\end{array}$ \\
\hline Perceived Control & & & Coping & & \\
\hline Control Beliefs & $-3.036 * *$ & -.13 & Problem-Solving & $-2.060^{*}$ & -.14 \\
\hline Total Strategy Beliefs & $-5.831 * * *$ & -.17 & Information-Seeking & .762 & .04 \\
\hline Effort & 1.426 & .10 & Escape & $-2.390^{*}$ & -.13 \\
\hline Ability & -1.029 & .07 & Confusion & -.059 & -.004 \\
\hline Powerful Others & $-3.981 * * *$ & -.22 & Engagement & & \\
\hline Luck & $-4.143^{* * *}$ & -.23 & Total Engagement & $-3.929 * * *$ & -.12 \\
\hline Unknown & $3.792^{* *}$ & .21 & Behavioral Engagement & $-2.701 * *$ & -.09 \\
\hline Total Capacity Beliefs & 1.710 & .06 & Emotional Engagement & $-3.564 * *$ & -.15 \\
\hline Effort & 1.014 & .04 & Teacher Support & & \\
\hline Ability & 1.654 & .08 & Total Teacher Support & $-3.731 * * *$ & -.08 \\
\hline Powerful Others & 1.596 & .09 & Structure & $-7.063^{* * *}$ & -.23 \\
\hline Luck & .111 & .01 & Involvement & -1.744 & -.05 \\
\hline Conmax & $3.393^{* *}$ & 3.71 & Autonomy Support & 1.323 & .04 \\
\hline
\end{tabular}


Table 23

Age Differences in the Influence of Ways of Coping on Engagement (Spring)

\begin{tabular}{|c|c|c|c|c|}
\hline Predictor (fall) & $\beta$ & $\mathrm{t}$ & $\mathrm{R}^{2}$ & F-Range \\
\hline Problem-Solving & .09 & $3.344 * * *$ & \multirow[t]{3}{*}{.15} & \multirow{3}{*}{$\begin{array}{c}29.370^{* * *} \\
- \\
46.565 * * *\end{array}$} \\
\hline Grade & -.249 & $-6.793 * * *$ & & \\
\hline Grade * Problem-Solving & .046 & 1.224 & & \\
\hline Information-Seeking & .127 & $4.938 * * *$ & \multirow[t]{3}{*}{.20} & \multirow{3}{*}{$\begin{array}{c}47.069^{* * *} \\
- \\
65.497^{* * *}\end{array}$} \\
\hline Grade & -.236 & $-6.693 * * *$ & & \\
\hline Grade * Info-Seeking & .058 & 1.622 & & \\
\hline Escape & -.234 & $-9.332 * * *$ & \multirow[t]{3}{*}{.33} & \multirow{3}{*}{$\begin{array}{c}91.960 * * * \\
- \\
128.671 * * *\end{array}$} \\
\hline Grade & -.179 & $-5.224 * * *$ & & \\
\hline Grade* Escape & .006 & .174 & & \\
\hline Confusion & -.164 & $-6.453 * * *$ & \multirow[t]{3}{*}{.24} & \multirow{3}{*}{$\begin{array}{c}58.769^{* * *} \\
- \\
82.908^{* * *}\end{array}$} \\
\hline Grade & -.250 & $-7.009 * * *$ & & \\
\hline Grade* Confusion & -.029 & -.745 & & \\
\hline
\end{tabular}


Table 24

Age Differences in the Contribution of Ways of Coping to Changes in Engagement from Fall-to-Spring - Mastery Coping

\begin{tabular}{lllll}
\hline Predictor (fall) & $\beta$ & $\mathrm{t}$ & $\Delta \mathrm{R}^{2}$ & F-Range \\
\hline
\end{tabular}

Engagement (spring)

\begin{tabular}{|c|c|c|c|c|}
\hline Engagement & .334 & $21.785^{* * *}$ & & \\
\hline Problem-Solving & .011 & .531 & .03 & $10.883 * * *-23.415 * * *$ \\
\hline Grade & -.156 & -5.443 & & \\
\hline Grade * Problem-Solving & -.037 & -1.228 & & \\
\hline
\end{tabular}

Engagement (spring)

\begin{tabular}{|c|c|c|c|c|}
\hline Engagement & .326 & $19.998 * * *$ & & \\
\hline Information-Seeking & .027 & 1.217 & .03 & $10.782 * * *-22.503 * * *$ \\
\hline Grade & -.155 & $-5.446^{* * *}$ & & \\
\hline Grade * Info-Seeking & -.027 & -1.009 & & \\
\hline
\end{tabular}


Table 25

Age Differences in the Contribution of Ways of Coping to Changes in Engagement from Fall-to-Spring - Helplessness Coping

\begin{tabular}{llccc}
\hline Predictor (fall) & $\beta$ & $\mathrm{t}$ & $\Delta \mathrm{R}^{2}$ & $\mathrm{~F}$ \\
\hline
\end{tabular}

Engagement (spring)

$\begin{array}{lccc}\text { Engagement } & .316 & 16.965^{* * *} & .03 \\ \text { Escape } & -.038 & -1.582 & 11.193^{* * *}-23.704^{* * *} \\ \text { Grade } & -.152 & -5.340 & \\ \text { Grade * Escape } & .030 & 1.037 & \end{array}$

Engagement (spring)

\begin{tabular}{|c|c|c|c|c|}
\hline Engagement & .311 & $19.461 * * *$ & & \\
\hline Confusion & -.035 & -1.632 & .04 & $13.142 * * *-29.222 * *$ \\
\hline Grade & -.158 & $-5.571 * * *$ & & \\
\hline Grade * Confusion & -.012 & .414 & & \\
\hline
\end{tabular}


Table 26

Age Differences in the Feedback Effects of Engagement on Ways of Coping

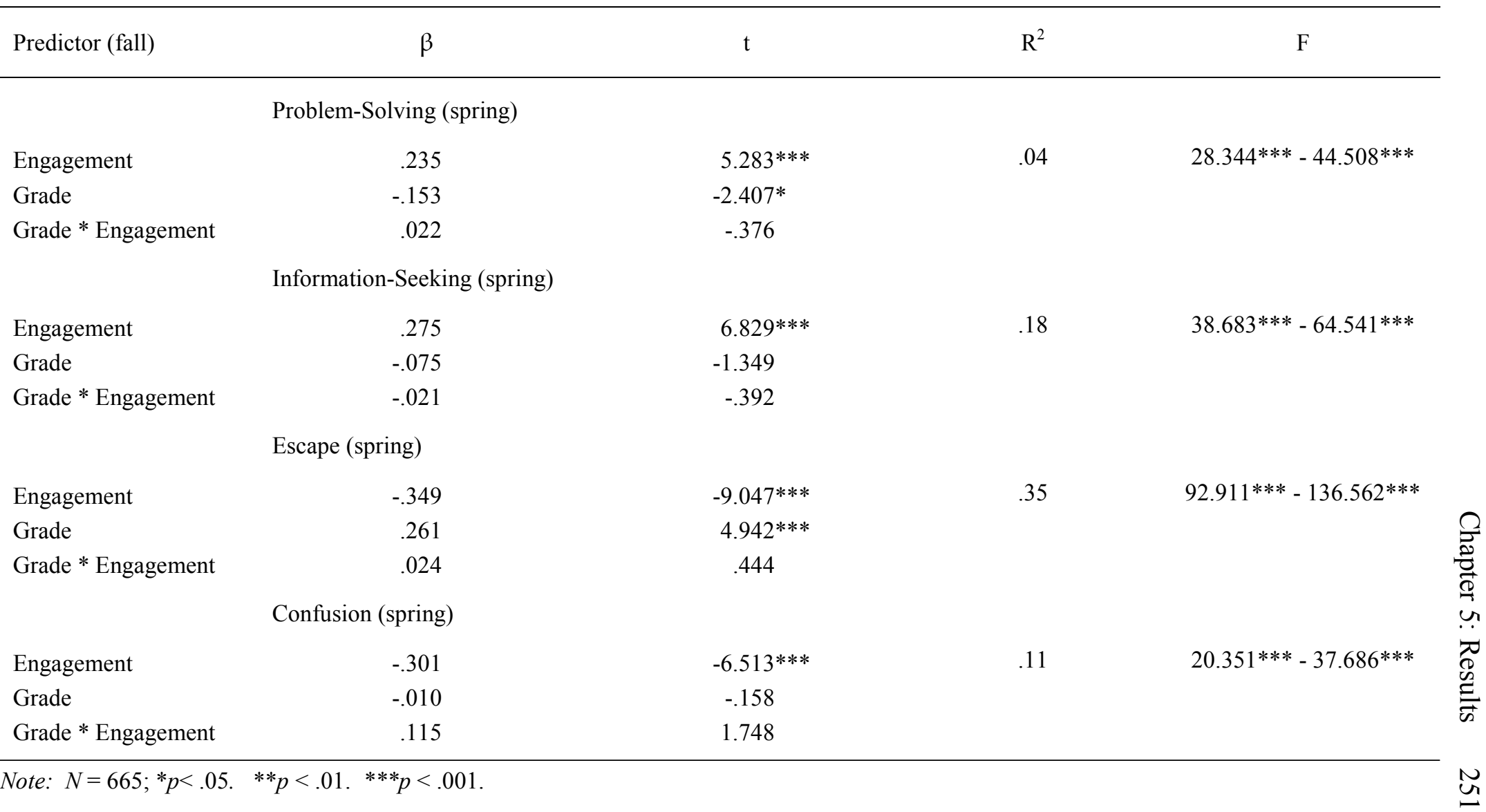


Table 27

Age Differences in the Influence of Ways of Coping on Achievement

\begin{tabular}{|c|c|c|c|c|c|c|c|c|}
\hline \multirow[t]{2}{*}{ Predictor (fall) } & \multicolumn{2}{|c|}{$\beta$} & \multicolumn{2}{|c|}{$\mathrm{t}$} & \multicolumn{2}{|c|}{$\mathrm{R}^{2}$} & \multicolumn{2}{|c|}{ F-Range } \\
\hline & Fall & Spring & Fall & Spring & Fall & Spring & Fall & Spring \\
\hline Problem-Solving & .098 & .150 & .855 & 1.382 & .01 & .02 & & \\
\hline Grade & .184 & .221 & 1.052 & 1.267 & & & .215 & 1.713 \\
\hline Grade* Problem-Solving & -.023 & .000 & -.157 & -.001 & & & $\begin{array}{c}- \\
7.630^{* * *}\end{array}$ & $10.489^{-} * * *$ \\
\hline Information-Seeking & .050 & .183 & .467 & 1.603 & .01 & .02 & & \\
\hline Grade & .181 & .208 & 1.041 & 1.187 & & & .032 & .947 \\
\hline Grade* Information-Seeking & .007 & -.072 & .047 & -.438 & & & $4.535^{* *}$ & $\stackrel{-}{9.552^{* * *}}$ \\
\hline Escape & -.286 & -.441 & $-1.965^{*}$ & $-3.553 * *$ & .05 & .07 & & \\
\hline Grade & .283 & .381 & 1.617 & $2.166^{*}$ & & & $3.337 *$ & $7.363 * * *$ \\
\hline Grade* Escape & -.018 & -.152 & -.105 & -.954 & & & $25.280^{-} * * *$ & $28.126^{* * *}$ \\
\hline Confusion & -.080 & -.085 & -.655 & -.733 & .02 & .02 & & \\
\hline Grade & .181 & .176 & 1.029 & .993 & & & .283 & .195 \\
\hline Grade*Confusion & -.063 & -.042 & -.391 & -.299 & & & $\begin{array}{c}- \\
7.464 * * *\end{array}$ & $\begin{array}{c}- \\
7.733^{*} * *\end{array}$ \\
\hline
\end{tabular}

Note: $N=665 ;{ }^{*} p<.05 .{ }^{* *} p<.01 .{ }^{* * *} p<.001$. 
Table 28

Age Differences in the Feedback Effects of Achievement on Ways of Coping

\begin{tabular}{|c|c|c|c|c|}
\hline Predictor (fall) & $\beta$ & $\mathrm{t}$ & $\mathrm{R}^{2}$ & F-Range \\
\hline & Problem-Solving (spring) & & & \\
\hline Achievement & .069 & .374 & .04 & $3.998 * *-17.167 * * *$ \\
\hline Grade & -.237 & $-3.803^{* * *}$ & & \\
\hline \multirow[t]{2}{*}{ Grade * Achievement } & .002 & .029 & & \\
\hline & Information-Seeking (spring) & & & \\
\hline Achievement & .078 & 1.583 & .03 & $3.657 *-12.544 * * *$ \\
\hline Grade & -.171 & $-2.954 * *$ & & \\
\hline \multirow[t]{2}{*}{ Grade * Achievement } & -.026 & -.363 & & \\
\hline & Escape (spring) & & & \\
\hline Achievement & -.159 & $-3.266^{* *}$ & .14 & $23.874 * * *-48.083^{* * *}$ \\
\hline Grade & .392 & $6.868^{* * *}$ & & \\
\hline \multirow[t]{2}{*}{ Grade * Achievement } & .014 & .204 & & \\
\hline & Confusion (spring) & & & \\
\hline Achievement & -.044 & -.727 & .01 & $.227-5.407 * *$ \\
\hline Grade & .077 & 1.145 & & \\
\hline Grade * Achievement & .021 & .281 & & \\
\hline
\end{tabular}

Note: $N=665 ;{ }^{*} p<.05 . \quad{ }^{* *} p<.01 .{ }^{* * *} p<.001$ 
Table 29

First and Second Stage Mediation Paths - Engagement as a Mediator of the Effects of Coping on Achievement: Problem-Solving (Fall)

\begin{tabular}{|c|c|c|c|c|c|c|}
\hline & $\mathrm{R}^{2}$ & & Range & $\mathrm{R}^{2}$ & \multicolumn{2}{|c|}{ F-Range } \\
\hline & .17 & \multicolumn{2}{|c|}{$36.809^{* * *}-53.657 * * *$} & .08 & \multicolumn{2}{|c|}{$3.520 * *-21.105 * * *$} \\
\hline Outcome: & \multicolumn{3}{|c|}{ Engagement (M) } & \multicolumn{3}{|c|}{ Achievement (Y) } \\
\hline Variable: & Notation & Estimate & $95 \% \mathrm{CI}$ & Notation & Estimate & $95 \% \mathrm{CI}$ \\
\hline Intercept & $i_{m} \rightarrow$ & $.151^{* *}$ & $(.051, .252)$ & $i_{y} \rightarrow$ & $8.042 * * *$ & $(7.776,8.308)$ \\
\hline Problem-Solving $(\mathrm{X})$ & $a_{1} \rightarrow$ & $.236^{* * *}$ & $(.132, .341)$ & $c_{1} \rightarrow$ & -.008 & $(-.250, .234)$ \\
\hline Engagement (M) & & & & $b_{1} \rightarrow$ & $.452 * *$ & $(.165, .738)$ \\
\hline Grade (W) & $a_{2} \rightarrow$ & $-.280^{* * *}$ & $(-.424,-.137)$ & $c_{2} \rightarrow$ & .292 & $(-.053, .637)$ \\
\hline $\mathrm{X} * \mathrm{~W}$ & $a_{3} \rightarrow$ & $.248 * *$ & $(.095, .402)$ & $c_{3} \rightarrow$ & -.064 & $(-.364, .235)$ \\
\hline $\mathrm{M} * \mathrm{~W}$ & & & & $b_{2} \rightarrow$ & -.148 & $(-.493, .197)$ \\
\hline
\end{tabular}


Table 30

First and Second Stage Mediation Paths - Engagement as a Mediator of the Effects of Coping on Achievement: Problem-Solving (Spring)

\begin{tabular}{|c|c|c|c|c|c|c|}
\hline & $\mathrm{R}^{2}$ & & Range & $\mathrm{R}^{2}$ & \multicolumn{2}{|c|}{ F-Range } \\
\hline & .25 & \multicolumn{2}{|c|}{$59.425 * * *-87.537 * * *$} & .06 & \multicolumn{2}{|c|}{$2.380 *-15.508 * * *$} \\
\hline Outcome: & \multicolumn{3}{|c|}{ Engagement (M) } & \multicolumn{3}{|c|}{ Achievement (Y) } \\
\hline Variable: & Notation & Estimate & $95 \% \mathrm{CI}$ & Notation & Estimate & $95 \% \mathrm{CI}$ \\
\hline Intercept & $i_{m} \rightarrow$ & $.229 * * *$ & $(.126, .331)$ & $i_{y} \rightarrow$ & $8.019 * * *$ & $(7.743,8.294)$ \\
\hline Problem-Solving $(\mathrm{X})$ & $a_{1} \rightarrow$ & $.375^{* * *}$ & $(.269, .480)$ & $c_{1} \rightarrow$ & .028 & $(-.192, .248)$ \\
\hline Engagement (M) & & & & $b_{1} \rightarrow$ & $.325^{*}$ & $(.054, .597)$ \\
\hline Grade $(\mathrm{W})$ & $a_{2} \rightarrow$ & $-.440 * * *$ & $(-.593,-.287)$ & $c_{2} \rightarrow$ & $.354^{*}$ & $(.001, .708)$ \\
\hline $\mathrm{X} * \mathrm{~W}$ & $a_{3} \rightarrow$ & .076 & $(-.082, .234)$ & $c_{3} \rightarrow$ & -.003 & $(-.334, .327)$ \\
\hline $\mathrm{M} * \mathrm{~W}$ & & & & $b_{2} \rightarrow$ & -.048 & $(-.384, .288)$ \\
\hline
\end{tabular}


Table 31

First and Second Stage Mediation Paths - Engagement as a Mediator of the Effects of Coping on Achievement: Information-Seeking (Fall)

\begin{tabular}{|c|c|c|c|c|c|c|}
\hline & $\mathrm{R}^{2}$ & & Range & $\mathrm{R}^{2}$ & \multicolumn{2}{|c|}{ F-Range } \\
\hline & .24 & \multicolumn{2}{|c|}{$56.832 * * *-85.329 * * *$} & .09 & \multicolumn{2}{|c|}{$3.696^{* *}-22.070^{* * *}$} \\
\hline Outcome: & \multicolumn{3}{|c|}{ Engagement (M) } & \multicolumn{3}{|c|}{ Achievement (Y) } \\
\hline Variable: & Notation & Estimate & $95 \% \mathrm{CI}$ & Notation & Estimate & $95 \% \mathrm{CI}$ \\
\hline Intercept & $i_{m} \rightarrow$ & $.139 * *$ & $(.042, .236)$ & $i_{y} \rightarrow$ & $8.046^{* * *}$ & $(7.781,8.310)$ \\
\hline Information-Seeking $(\mathrm{X})$ & $a_{1} \rightarrow$ & $.307^{* * *}$ & $(.204, .411)$ & $c_{1} \rightarrow$ & -.097 & $(-.318, .123)$ \\
\hline Engagement (M) & & & & $b_{1} \rightarrow$ & $.481 * * *$ & $(.199, .764)$ \\
\hline Grade $(\mathrm{W})$ & $a_{2} \rightarrow$ & $-.250 * * *$ & $(-.389,-.110)$ & $c_{2} \rightarrow$ & .288 & $(-.056, .631)$ \\
\hline $\mathrm{X} * \mathrm{~W}$ & $a_{3} \rightarrow$ & $.260 * * *$ & $(.118, .407)$ & $c_{3} \rightarrow$ & -.046 & $(-.360, .269)$ \\
\hline $\mathrm{M} * \mathrm{~W}$ & & & & $b_{2} \rightarrow$ & -.130 & $(-.480, .220)$ \\
\hline
\end{tabular}


Table 32

First and Second Stage Mediation Paths - Engagement as a Mediator of the Effects of Coping on Achievement: Information-Seeking (Spring)

\begin{tabular}{|c|c|c|c|c|c|c|}
\hline & $\mathrm{R}^{2}$ & & Range & $\mathrm{R}^{2}$ & \multicolumn{2}{|c|}{ F-Range } \\
\hline & .29 & \multicolumn{2}{|c|}{$69.126^{* * *}-105.502 * * *$} & .06 & \multicolumn{2}{|c|}{$2.763^{*}-14.804^{* * *}$} \\
\hline Outcome: & \multicolumn{3}{|c|}{ Engagement (M) } & \multicolumn{3}{|c|}{ Achievement (Y) } \\
\hline Variable: & Notation & Estimate & $95 \% \mathrm{CI}$ & Notation & Estimate & $95 \% \mathrm{CI}$ \\
\hline Intercept & $i_{m} \rightarrow$ & $.239 * * *$ & $(.139, .339)$ & $i_{y} \rightarrow$ & $8.020 * * *$ & $(7.744,8.297)$ \\
\hline Information-Seeking (X) & $a_{1} \rightarrow$ & $.420 * * *$ & $(.319, .522)$ & $c_{1} \rightarrow$ & .050 & $(-.190, .289)$ \\
\hline Engagement (M) & & & & $b_{1} \rightarrow$ & $.314^{*}$ & $(.033, .595)$ \\
\hline Grade (W) & $a_{2} \rightarrow$ & $-.463 * * *$ & $(-.614,-.311)$ & $c_{2} \rightarrow$ & .352 & $(-.005, .709)$ \\
\hline $\mathrm{X} * \mathrm{~W}$ & $a_{3} \rightarrow$ & .080 & $(-.705, .235)$ & $c_{3} \rightarrow$ & -.092 & $(-.434, .249)$ \\
\hline $\mathrm{M} * \mathrm{~W}$ & & & & $b_{2} \rightarrow$ & -.005 & $(-.360, .350)$ \\
\hline
\end{tabular}


Table 33

First and Second Stage Mediation Paths - Engagement as a Mediator of the Effects of Coping on Achievement: Escape (Fall)

\begin{tabular}{|c|c|c|c|c|c|c|}
\hline & $\mathrm{R}^{2}$ & & Range & $\mathrm{R}^{2}$ & \multicolumn{2}{|c|}{ F-Range } \\
\hline & .45 & \multicolumn{2}{|c|}{$163.698 * * *-209.256 * * *$} & .09 & \multicolumn{2}{|c|}{$3.607 * *-22.407 * * *$} \\
\hline Outcome: & \multicolumn{3}{|c|}{ Engagement (M) } & \multicolumn{3}{|c|}{ Achievement (Y) } \\
\hline Variable: & Notation & Estimate & $95 \% \mathrm{CI}$ & Notation & Estimate & $95 \% \mathrm{CI}$ \\
\hline Intercept & $i_{m} \rightarrow$ & .050 & $(-.035, .136)$ & $i_{y} \rightarrow$ & $8.041 * * *$ & $(7.770,8.311)$ \\
\hline Escape $(\mathrm{X})$ & $a_{1} \rightarrow$ & $-.620 * * *$ & $(-.725,-.516)$ & $c_{1} \rightarrow$ & -.010 & $(-.327, .306)$ \\
\hline Engagement $(\mathrm{M})$ & & & & $b_{1} \rightarrow$ & $.444 * *$ & $(.157, .731)$ \\
\hline Grade $(W)$ & $a_{2} \rightarrow$ & -.085 & $(-.210, .039)$ & $c_{2} \rightarrow$ & .309 & $(-.036, .655)$ \\
\hline $\mathrm{X} * \mathrm{~W}$ & $a_{3} \rightarrow$ & -.077 & $(-.201, .048)$ & $c_{3} \rightarrow$ & -.219 & $(-.650, .212)$ \\
\hline $\mathrm{M} * \mathrm{~W}$ & & & & $b_{2} \rightarrow$ & -.337 & $(-.756, .081)$ \\
\hline
\end{tabular}


Table 34

First and Second Stage Mediation Paths - Engagement as a Mediator of the Effects of Coping on Achievement: Escape (Spring)

\begin{tabular}{|c|c|c|c|c|c|c|}
\hline & $\mathrm{R}^{2}$ & & Range & $\mathrm{R}^{2}$ & \multicolumn{2}{|c|}{ F-Range } \\
\hline & .46 & \multicolumn{2}{|c|}{$149.830 * * *-226.326 * * *$} & .08 & \multicolumn{2}{|c|}{$4.853 * * *-20.309 * * *$} \\
\hline Outcome: & \multicolumn{3}{|c|}{ Engagement $(\mathrm{M})$} & \multicolumn{3}{|c|}{ Achievement (Y) } \\
\hline Variable: & Notation & Estimate & $95 \% \mathrm{CI}$ & Notation & Estimate & $95 \% \mathrm{CI}$ \\
\hline Intercept & $i_{m} \rightarrow$ & $.101^{*}$ & $(.001, .202)$ & $i_{y} \rightarrow$ & $7.980 * * *$ & $(7.704,8.256)$ \\
\hline Escape (X) & $a_{1} \rightarrow$ & $-.657 * * *$ & $(-.765,-.548)$ & $c_{1} \rightarrow$ & $-.381 *$ & $(-.707,-.055)$ \\
\hline Engagement (M) & & & & $b_{1} \rightarrow$ & .099 & $(-.240, .438)$ \\
\hline Grade $(\mathrm{W})$ & $a_{2} \rightarrow$ & $-.210^{*}$ & $(-.359,-.060)$ & $c_{2} \rightarrow$ & $.412 *$ & $(.055, .769)$ \\
\hline $\mathrm{X} * \mathrm{~W}$ & $a_{3} \rightarrow$ & .029 & $(-.132, .191)$ & $c_{3} \rightarrow$ & .203 & $(-.212, .618)$ \\
\hline $\mathrm{M} * \mathrm{~W}$ & & & & $b_{2} \rightarrow$ & .079 & $(-.361, .520)$ \\
\hline
\end{tabular}


Table 35

First and Second Stage Mediation Paths - Engagement as a Mediator of the Effects of Coping on Achievement: Confusion (Fall)

\begin{tabular}{|c|c|c|c|c|c|c|}
\hline & $\mathrm{R}^{2}$ & & Range & $\mathrm{R}^{2}$ & \multicolumn{2}{|c|}{ F-Range } \\
\hline & .22 & \multicolumn{2}{|c|}{$55.701 * * *-72.596 * * *$} & .08 & \multicolumn{2}{|c|}{$3.892 * *-20.788 * * *$} \\
\hline Outcome: & \multicolumn{3}{|c|}{ Engagement $(\mathrm{M})$} & \multicolumn{3}{|c|}{ Achievement (Y) } \\
\hline Variable: & Notation & Estimate & $95 \% \mathrm{CI}$ & Notation & Estimate & $95 \% \mathrm{CI}$ \\
\hline Intercept & $i_{m} \rightarrow$ & $.150 *$ & $(.052, .248)$ & $i_{y} \rightarrow$ & $8.037 * * *$ & $(7.771,8.303)$ \\
\hline Confusion (X) & $a_{1} \rightarrow$ & $-.451 * * *$ & $(-.515,-.316)$ & $c_{1} \rightarrow$ & .133 & $(-.108, .374)$ \\
\hline Engagement $(\mathrm{M})$ & & & & $b_{1} \rightarrow$ & $.513^{* * *}$ & $(.241, .785)$ \\
\hline Grade $(\mathrm{W})$ & $a_{2} \rightarrow$ & $-.295 * * *$ & $(-.435,-.154)$ & $c_{2} \rightarrow$ & .297 & $(-.047, .640)$ \\
\hline $\mathrm{X} * \mathrm{~W}$ & $a_{3} \rightarrow$ & -.052 & $(-.204, .100)$ & $c_{3} \rightarrow$ & -.153 & $(-.491, .184)$ \\
\hline $\mathrm{M} * \mathrm{~W}$ & & & & $b_{2} \rightarrow$ & -.249 & $(-.586, .088)$ \\
\hline
\end{tabular}


Table 36

First and Second Stage Mediation Paths - Engagement as a Mediator of the Effects of Coping on Achievement: Confusion (Spring)

\begin{tabular}{|c|c|c|c|c|c|c|}
\hline & $\mathrm{R}^{2}$ & & Range & $\mathrm{R}^{2}$ & \multicolumn{2}{|c|}{ F-Range } \\
\hline & .24 & \multicolumn{2}{|c|}{$55.839^{* * *}-90.496^{* * *}$} & .06 & \multicolumn{2}{|c|}{$2.948 *-15.170 * * *$} \\
\hline Outcome: & \multicolumn{3}{|c|}{ Engagement (M) } & \multicolumn{3}{|c|}{ Achievement (Y) } \\
\hline Variable: & Notation & Estimate & $95 \% \mathrm{CI}$ & Notation & Estimate & $95 \% \mathrm{CI}$ \\
\hline Intercept & $i_{m} \rightarrow$ & $.272 * * *$ & $(.169, .374)$ & $i_{y} \rightarrow$ & $8.011 * * *$ & $(7.733,8.288)$ \\
\hline Confusion (X) & $a_{1} \rightarrow$ & $-.440 * * *$ & $(-.548,-.335)$ & $c_{1} \rightarrow$ & .083 & $(-.149, .315)$ \\
\hline Engagement (M) & & & & $b_{1} \rightarrow$ & $.382 * *$ & $(.110, .654)$ \\
\hline Grade $(\mathrm{W})$ & $a_{2} \rightarrow$ & $-.543 * * *$ & $(-.696,-.389)$ & $c_{2} \rightarrow$ & $.364 *$ & $(.010, .718)$ \\
\hline $\mathrm{X} * \mathrm{~W}$ & $a_{3} \rightarrow$ & .103 & $(-.063, .268)$ & $c_{3} \rightarrow$ & -.021 & $(-.312, .269)$ \\
\hline $\mathrm{M} * \mathrm{~W}$ & & & & $b_{2} \rightarrow$ & -.074 & $(-.434, .286)$ \\
\hline
\end{tabular}


Table 37

Significance Test of the Indirect Effect of Coping on Achievement, as Mediated Through Engagement

\begin{tabular}{|c|c|c|c|c|c|c|}
\hline \multirow[b]{2}{*}{$4^{\text {th }}$ Grade } & \multicolumn{2}{|c|}{ Estimate } & \multicolumn{2}{|c|}{ SE } & \multicolumn{2}{|c|}{$95 \% \mathrm{CI}$} \\
\hline & Fall & Spring & Fall & Spring & Fall & Spring \\
\hline Problem-Solving & $.106^{*}$ & $.122 *$ & .043 & .059 & $(.024, .188)$ & $(.007, .237)$ \\
\hline Information-Seeking & $.148 * *$ & .133 & .052 & .069 & $(.045, .250)$ & $(-.001, .268)$ \\
\hline Escape & $.010 * *$ & -.066 & .096 & .121 & $(-.464,-.087)$ & $(-.302, .171)$ \\
\hline Confusion & $-.213^{* *}$ & $-.168 *$ & .066 & .067 & $(-.342,-.085)$ & $(-.299,-.037)$ \\
\hline
\end{tabular}

6th Grade

\begin{tabular}{|c|c|c|c|c|c|c|}
\hline Problem-Solving & $.151^{*}$ & $.125^{*}$ & .059 & .057 & $(.032, .263)$ & $(.014, .237)$ \\
\hline Information-Seeking & $.200^{*}$ & $.154^{*}$ & .067 & .060 & $(.069, .331)$ & $(.036, .272)$ \\
\hline Escape & -.074 & -.112 & .107 & .093 & $(-.284, .136)$ & $(-.294, .070)$ \\
\hline Confusion & $-.124 *$ & $-.104 *$ & .057 & .048 & $(-.236,-.011)$ & $(-.198,-.011)$ \\
\hline
\end{tabular}

Note: $N=665 ;{ }^{*} p<.05 .{ }^{* *} p<.01 .{ }^{* * *} p<.001$. 
Table 38

Moderation of the Indirect Effect of Coping on Achievement, as Mediated Through Engagement

\begin{tabular}{|c|c|c|c|}
\hline & $\begin{array}{l}\text { Index of Mediated Moderation } \\
\text { (IMM) }\end{array}$ & SE & $95 \% \mathrm{CI}$ \\
\hline \multicolumn{4}{|l|}{ Fall Models } \\
\hline Problem-Solving & .041 & .070 & $(-.095, .178)$ \\
\hline Information-Seeking & .052 & .082 & $(-.108, .212)$ \\
\hline Escape & .201 & .143 & $(-.079, .482)$ \\
\hline Confusion & .090 & .082 & $(-.072, .251)$ \\
\hline \multicolumn{4}{|l|}{ Spring Models } \\
\hline Problem-Solving & .003 & .078 & $(-.150, .156)$ \\
\hline Information-Seeking & .021 & .091 & $(-.157, .198)$ \\
\hline Escape & -.047 & .151 & $(-.342, .249)$ \\
\hline Confusion & .064 & .082 & $(-.096, .223)$ \\
\hline
\end{tabular}


Table 39

Control Aggregates Predicting Ways of Coping — Mastery Coping

\begin{tabular}{|c|c|c|c|c|c|c|c|c|c|c|}
\hline \multirow[b]{2}{*}{ Predictor (fall) } & \multicolumn{2}{|c|}{$\beta$} & \multicolumn{2}{|c|}{$\mathrm{t}$} & \multicolumn{2}{|c|}{$\mathrm{R}^{2}$} & \multicolumn{2}{|c|}{ F-Range } & \multicolumn{2}{|c|}{$\begin{array}{l}\text { Semi-Partial } \\
\text { Correlation }\end{array}$} \\
\hline & Fall & Spring & Fall & Spring & Fall & Spring & Fall & Spring & Fall & Spring \\
\hline \multicolumn{11}{|c|}{ Problem-Solving } \\
\hline Total Strategy Beliefs & -.107 & -.185 & $-3.234 * *$ & $-4.563 * * *$ & & & $16.187 * * *$ & $25.816^{* * *}$ & -.13 & -.19 \\
\hline Grade & -.133 & -.188 & $-2.884 * *$ & $-3.076 * * *$ & .09 & .13 & - & - & -.12 & -.14 \\
\hline Grade $*$ Tot Strat Beliefs & -.101 & -.055 & $-2.138^{*}$ & -.995 & & & $25.978 * * *$ & $42.779 * * *$ & -.09 & -.04 \\
\hline Total Capacity Beliefs & .186 & .252 & $5.166^{* * *}$ & $6.235^{* * *}$ & & & $28.147 * * *$ & $32.601 * * *$ & .20 & .25 \\
\hline Grade & .010 & -.088 & .206 & -1.389 & .13 & .15 & - & - & .01 & -.06 \\
\hline Grade $*$ Tot Cap Beliefs & .037 & -.013 & .792 & -.228 & & & $40.271 * * *$ & $48.476^{* * *}$ & .03 & -.01 \\
\hline \multicolumn{11}{|c|}{ Information-Seeking } \\
\hline Total Strategy Beliefs & -.070 & -.160 & $-2.011^{*}$ & $-3.779 * * *$ & & & $11.813 * * *$ & $17.013 * * *$ & -.08 & -.17 \\
\hline Grade & -.155 & -.129 & $-3.190 * *$ & $-2.264^{*}$ & .07 & .10 & - & - & -.13 & -.10 \\
\hline Grade $*$ Tot Strat Beliefs & -.125 & -.042 & $-2.554^{*}$ & -.737 & & & $19.544 * * *$ & $33.900 * * *$ & -.10 & -.03 \\
\hline Total Capacity Beliefs & .276 & .298 & $7.404 * * *$ & $7.767 * * *$ & & & $48.777 * * *$ & $44.417 * * *$ & .29 & .31 \\
\hline Grade & .015 & -.004 & .327 & -.065 & .21 & .19 & - & $\overline{-}$ & .01 & -.003 \\
\hline Grade $*$ Tot Cap Beliefs & -.001 & -.036 & -.024 & -.669 & & & $67.847 * * *$ & $64.111^{* * *}$ & -.001 & -.03 \\
\hline
\end{tabular}

Note: $N=665 ;{ }^{*} p<.05 .{ }^{* *} p<.01 .{ }^{* * *} p<.001$. 
Table 40

Control Aggregates Predicting Ways of Coping - Helplessness Coping

\begin{tabular}{|c|c|c|c|c|c|c|c|c|c|c|}
\hline \multirow[b]{2}{*}{ Predictor (fall) } & \multicolumn{2}{|c|}{$\beta$} & \multicolumn{2}{|c|}{$\mathrm{t}$} & \multicolumn{2}{|c|}{$\mathrm{R}^{2}$} & \multicolumn{2}{|c|}{ F-Range } & \multicolumn{2}{|c|}{$\begin{array}{c}\text { Semi-Partial } \\
\text { Correlation }\end{array}$} \\
\hline & Fall & Spring & Fall & Spring & Fall & Spring & Fall & Spring & Fall & Spring \\
\hline \multicolumn{11}{|c|}{ Escape } \\
\hline Total Strategy Beliefs & .208 & .283 & $6.088 * * *$ & $7.503 * * *$ & \multirow[b]{2}{*}{.20} & \multirow[b]{2}{*}{.34} & \multirow{2}{*}{$\begin{array}{c}45.958 * * * \\
- \\
63.171 * * *\end{array}$} & \multirow{2}{*}{$\begin{array}{c}92.909 * * * \\
- \\
146.894 * * *\end{array}$} & .23 & .30 \\
\hline Grade & .308 & .313 & $6.564 * * *$ & $6.126^{* * *}$ & & & & & .24 & .24 \\
\hline Total Capacity Beliefs & -.384 & -.381 & $-11.265^{* * *}$ & $-10.201 * * *$ & & & $125.240 * * *$ & $129.288 * * *$ & -.38 & -.39 \\
\hline Grade & .052 & .157 & 1.211 & $2.880^{* *}$ & .39 & .40 & - & - & .04 & .12 \\
\hline \multirow[t]{2}{*}{ Grade $*$ Tot Cap Beliefs } & -.001 & .002 & -.014 & .046 & & & $155.547 * * *$ & $175.749 * * *$ & -.001 & .002 \\
\hline & \multicolumn{2}{|c|}{ Confusion } & & & & & & & & \\
\hline Total Strategy Beliefs & .107 & .174 & $2.570 *$ & $3.679 * * *$ & \multirow{3}{*}{.03} & \multirow{3}{*}{.04} & \multirow{3}{*}{$\begin{array}{c}3.471 * \\
- \\
7.794 * * *\end{array}$} & \multirow{3}{*}{$\begin{array}{c}7.038 * * * \\
- \\
15.647 * * *\end{array}$} & .11 & .16 \\
\hline Grade & .097 & .044 & 1.704 & .661 & & & & & .07 & .03 \\
\hline Grade $*$ Tot Strat Beliefs & .005 & -.054 & .088 & -.833 & & & & & .004 & -.04 \\
\hline Total Capacity Beliefs & -.290 & -.354 & $-6.645 * * *$ & $-7.808 * * *$ & & & $30.429 * * *$ & $33.284 * * *$ & -.26 & -.32 \\
\hline Grade & -.069 & -.102 & -1.229 & -1.542 & .15 & .16 & - & - & -.05 & -.07 \\
\hline Grade $*$ Tot Cap Beliefs & .026 & .099 & .463 & 1.615 & & & $43.202 * * *$ & $58.743 * * *$ & .02 & .07 \\
\hline
\end{tabular}

Note: $N=665 ; * p<.05 . * * p<.01 . * * * p<.001$. 
Table 41

Control Components (Strategy) Predicting Ways of Coping - Problem-Solving (Fall)

\begin{tabular}{|c|c|c|c|c|c|}
\hline Predictor (fall) & $\beta$ & $\mathrm{t}$ & $\mathrm{R}^{2}$ & F-Range & $\begin{array}{c}\text { Semi-Partial } \\
\text { Correlation }\end{array}$ \\
\hline Strategy Beliefs & & & . & & \\
\hline Effort & .083 & $2.259^{*}$ & 11 & $5.934 * * *-8.776 * * *$ & .10 \\
\hline Ability & .063 & 1.815 & & & .07 \\
\hline Powerful Others & -.005 & -.132 & & & -.01 \\
\hline Luck & -.041 & -1.017 & & & -.04 \\
\hline Unknown & -.032 & -.818 & & & -.03 \\
\hline Grade & -.143 & $-3.003 * *$ & & & -.12 \\
\hline Grade * Effort & .009 & .163 & & & .01 \\
\hline Grade * Ability & -.047 & -.910 & & & -.04 \\
\hline Grade * Pow. Others & -.080 & -1.408 & & & -.06 \\
\hline Grade $*$ Luck & -.089 & -1.545 & & & -.06 \\
\hline Grade * Unknown & -.004 & -.078 & & & -.003 \\
\hline Note: $N=664 ;{ }^{*} p<.05$. & $* * * p$ & & & & \\
\hline
\end{tabular}


Table 42

Control Components (Strategy) Predicting Ways of Coping - Information-Seeking (Fall)

\begin{tabular}{|c|c|c|c|c|c|}
\hline Predictor (fall) & $\beta$ & $\mathrm{t}$ & $\mathrm{R}^{2}$ & F-Range & $\begin{array}{l}\text { Semi-Partial } \\
\text { Correlation }\end{array}$ \\
\hline \multicolumn{6}{|l|}{ Strategy Beliefs } \\
\hline Effort & .049 & 1.327 & .11 & $5.939 * * *-8.435 * * *$ & .05 \\
\hline Ability & -.026 & -.698 & & & -.03 \\
\hline Powerful Others & -.048 & -1.124 & & & -.05 \\
\hline Luck & -.029 & -.700 & & & -.03 \\
\hline Unknown & -.018 & -.455 & & & -.02 \\
\hline Grade & -.134 & $-2.707 * *$ & & & -.11 \\
\hline Grade * Effort & -.049 & -.945 & & & -.04 \\
\hline Grade * Ability & .033 & .615 & & & -.03 \\
\hline Grade * Pow. Others & -.068 & -1.120 & & & -.05 \\
\hline Grade $*$ Luck & -.065 & -1.046 & & & -.04 \\
\hline Grade * Unknown & -.078 & -1.378 & & & -.05 \\
\hline \multicolumn{6}{|c|}{ Note: $N=664 ;{ }^{*} p<.05 .{ }^{* *} p<.01 .{ }^{* * *} p<.001$} \\
\hline
\end{tabular}


Table 43

Age Differences in the Contribution of Maximum Control to Changes in Coping from Fall-to-Spring - Mastery Coping

\begin{tabular}{llccc}
\hline Predictor (fall) & $\beta$ & $\mathrm{t}$ & $\Delta \mathrm{R}^{2}$ & F-Range \\
\hline
\end{tabular}

Problem-Solving (spring)

\begin{tabular}{|c|c|c|c|c|}
\hline Problem-Solving & .413 & $6.376^{* * *}$ & & \\
\hline Conmax & .119 & $2.520^{*}$ & .04 & $73.488^{* * *}-171.205^{* * *}$ \\
\hline Grade & -.165 & $-2.663 * *$ & & \\
\hline Grade $*$ Conmax & -.062 & -1.065 & & \\
\hline
\end{tabular}

Information-Seeking (spring)

\begin{tabular}{|c|c|c|c|c|}
\hline Information-Seeking & .492 & $10.951 * * *$ & & \\
\hline Conmax & .115 & $2.878^{* *}$ & .02 & $3.224 *-11.227 * * *$ \\
\hline Grade & -.078 & -1.497 & & \\
\hline Grade * Conmax & -.048 & -.941 & & \\
\hline
\end{tabular}


Table 44

Age Differences in the Contribution of Maximum Control to Changes in Coping from Fall-to-Spring - Helplessness Coping

\begin{tabular}{lllll}
\hline Predictor (fall) & $\beta$ & $\mathrm{t}$ & $\Delta \mathrm{R}^{2}$ & F-Range \\
\hline
\end{tabular}

Escape (spring)

\begin{tabular}{|c|c|c|c|c|}
\hline Escape & .358 & $6.678 * * *$ & & \\
\hline Conmax & -.198 & $-4.703 * * *$ & .08 & $20.990 * * *-38.432 * * *$ \\
\hline Grade & .237 & $4.635^{* * *}$ & & \\
\hline Grade *Conmax & .041 & .852 & & \\
\hline
\end{tabular}

Confusion (spring)

\begin{tabular}{|c|c|c|c|c|}
\hline Confusion & .518 & $11.458 * * *$ & & \\
\hline Conmax & -.166 & $-3.638 * * *$ & .02 & $2.545-14.108^{* * *}$ \\
\hline Grade & .004 & .063 & & \\
\hline Grade *Conmax & .102 & 1.826 & & \\
\hline
\end{tabular}


Table 45

Age Differences in the Contribution of Teacher Support to Ways of Coping - Mastery Coping

\begin{tabular}{llllllllllll}
\hline & \multicolumn{2}{c}{$\beta$} & & \multicolumn{2}{c}{$\mathrm{t}$} & & \multirow{2}{*}{$\mathrm{R}^{2}$} & & \multicolumn{2}{c}{ F-Range } & \multicolumn{2}{c}{$\begin{array}{c}\text { Semi-Partial } \\
\text { Correlation }\end{array}$} \\
\hline Predictor & Fall & Spring & Fall & & Spring & Fall & Spring & Fall & Spring & Fall & Spring \\
\hline
\end{tabular}

Problem-Solving

\begin{tabular}{|c|c|c|c|c|c|c|c|c|c|c|}
\hline Total Teacher Support & .050 & .064 & 1.460 & 1.237 & .02 & .05 & $\begin{array}{c}2.007 \\
-\end{array}$ & $\begin{array}{c}6.930^{* * *} \\
-\end{array}$ & .06 & .06 \\
\hline Grade & -.071 & -.216 & -1.459 & $-3.384 * *$ & & & $4.652 * *$ & $15.350 * * *$ & -.06 & -.16 \\
\hline Grade * Tot Tch Support & .002 & .027 & .046 & .404 & & & & & .002 & .02 \\
\hline
\end{tabular}

Information-Seeking

\begin{tabular}{|c|c|c|c|c|c|c|c|c|c|c|}
\hline Total Teacher Support & .045 & .077 & 1.243 & 1.607 & .03 & .03 & $\begin{array}{c}3.414 * \\
-\end{array}$ & $\begin{array}{c}5.505^{* *} \\
-\end{array}$ & .05 & .08 \\
\hline Grade & -.093 & -.151 & -1.852 & $-2.582^{*}$ & & & $7.817 * * *$ & $13.154 * * *$ & -.08 & -.12 \\
\hline Grade * Tot Tch Support & .049. & .006 & .977 & .099 & & & & & .04 & .01 \\
\hline Note: $N=665 ; * p<.05$ & .01. " & <.001. & & & & & & & & \\
\hline
\end{tabular}


Table 46

Age Differences in the Contribution of Teacher Support to Ways of Coping-Helplessness Coping

\begin{tabular}{llllllllllll}
\hline & \multicolumn{2}{c}{$\beta$} & & \multicolumn{2}{c}{$\mathrm{t}$} & & \multirow{2}{*}{$\mathrm{R}^{2}$} & & \multicolumn{2}{c}{ F-Range } & \multicolumn{2}{c}{$\begin{array}{c}\text { Semi-Partial } \\
\text { Correlation }\end{array}$} \\
\hline Predictor & Fall & Spring & Fall & & Spring & Fall & Spring & Fall & Spring & Fall & Spring \\
\hline
\end{tabular}

Escape

$\begin{array}{llll}-.140 & -.163 & -3.721 * * * & -3.743 * * * \\ .181 & .353 & 3.595 * * * & 6.195 * * * \\ -.025 & .044 & -.462 & .797\end{array}$

.13

$18.633 * * * \quad 29.397 * * *$

$-.15-.165$

Grade

$.044-.462-.797$

$26.124 * * * \quad 40.290 * * * \quad .14 \quad .273$

Grade * Tot Tch Support

Confusion

Confusion

\begin{tabular}{|c|c|c|c|c|c|c|c|c|c|c|}
\hline Total Teacher Support & -.017 & -.085 & -.378 & -.1651 & .01 & .02 & $\begin{array}{c}1.180 \\
-\end{array}$ & $\begin{array}{c}1.420 \\
-\end{array}$ & -.02 & -.08 \\
\hline Grade & .050 & .059 & .853 & .884 & & & $3.516^{* *}$ & $6.799 * * *$ & .04 & .04 \\
\hline Grade * Tot Tch Support & -.060 & .003 & -.946 & .038 & & & & & -.04 & .002 \\
\hline
\end{tabular}




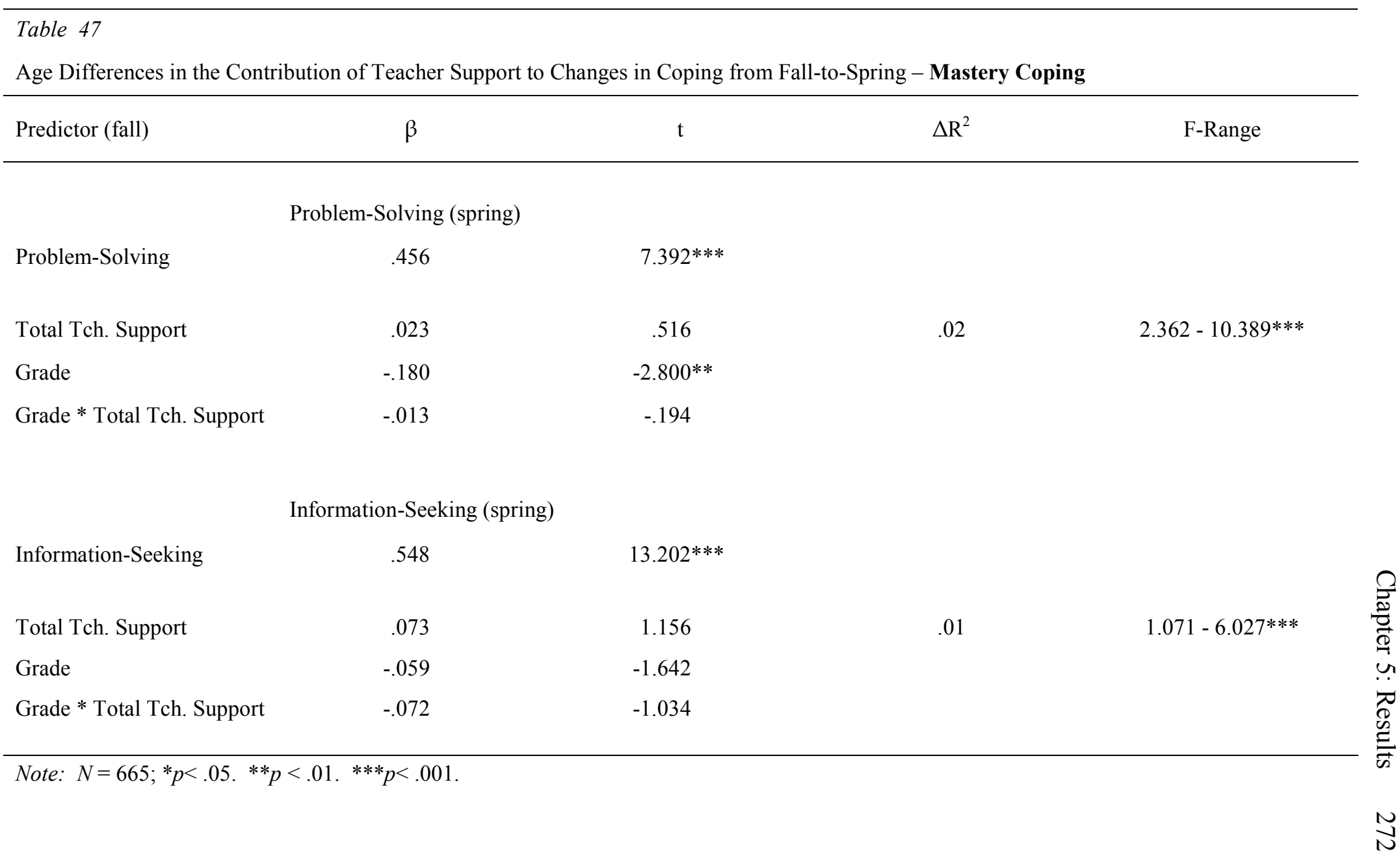


Table 48

Age Differences in the Contribution of Teacher Support to Changes in Coping from Fall-to-Spring - Helplessness Coping

\begin{tabular}{ccccc}
\hline Predictor (fall) & $\beta$ & $\mathrm{t}$ & $\Delta \mathrm{R}^{2}$ & F-Range \\
\hline
\end{tabular}

Escape (spring)

\begin{tabular}{|c|c|c|c|c|}
\hline Escape & .538 & $12.514 * * *$ & & \\
\hline Total Tch. Support & -.040 & -1.114 & .04 & $7.360 * * *-21.683^{* * *}$ \\
\hline Grade & .244 & $4.528 * * *$ & & \\
\hline Grade * Total Tch. Support & .078 & 1.582 & & \\
\hline
\end{tabular}

Confusion (spring)

\begin{tabular}{|c|c|c|c|c|}
\hline Confusion & .582 & $14.167 * * *$ & & \\
\hline Total Tch. Support & -.030 & -.719 & .00 & $.301-2.929 *$ \\
\hline Grade & .021 & .333 & & \\
\hline Grade * Total Tch. Support & .002 & .028 & & \\
\hline
\end{tabular}

Note: $N=665 ;{ }^{*} p<.05 .{ }^{* *} p<.01 .{ }^{* * *} p<.001$. 
Table 49

Age Differences in the Feedback Effects of Ways of Coping on Changes in Total Strategy Beliefs - Mastery Coping

\begin{tabular}{llcl}
\hline Predictor (fall) & $\beta$ & $\mathrm{t}$ & $\Delta \mathrm{R}^{2}$ \\
\hline
\end{tabular}

Total Strategy Beliefs (spring)

\begin{tabular}{|c|c|c|c|c|}
\hline Total Strategy Beliefs & .215 & $16.048 * * *$ & & \\
\hline Problem-Solving & -.008 & -.431 & .03 & $7.827 * * *-14.158 * * *$ \\
\hline Grade & .128 & $4.878 * * *$ & & \\
\hline Grade * Problem-Solving & .020 & .690 & & \\
\hline Total Strategy Beliefs & .210 & $15.624 * * *$ & & \\
\hline Information-Seeking & -.023 & -1.276 & .03 & $6.594 * * *-14.490 * * *$ \\
\hline Grade & .123 & $4.674 * * *$ & & \\
\hline Grade * Info-Seeking & .009 & .362 & & \\
\hline \multicolumn{5}{|c|}{ Note: $N=665 ; * p<.05 . \quad * * p<.01 . * * * p<.001$. } \\
\hline
\end{tabular}


Table 50

Age Differences in the Feedback Effects of Ways of Coping on Changes in Total Strategy Beliefs - Helplessness Coping

\begin{tabular}{lllll}
\hline Predictor (fall) & $\beta$ & $\mathrm{t}$ & $\Delta \mathrm{R}^{2}$ & F-Range \\
\hline
\end{tabular}

Total Strategy Beliefs (spring)

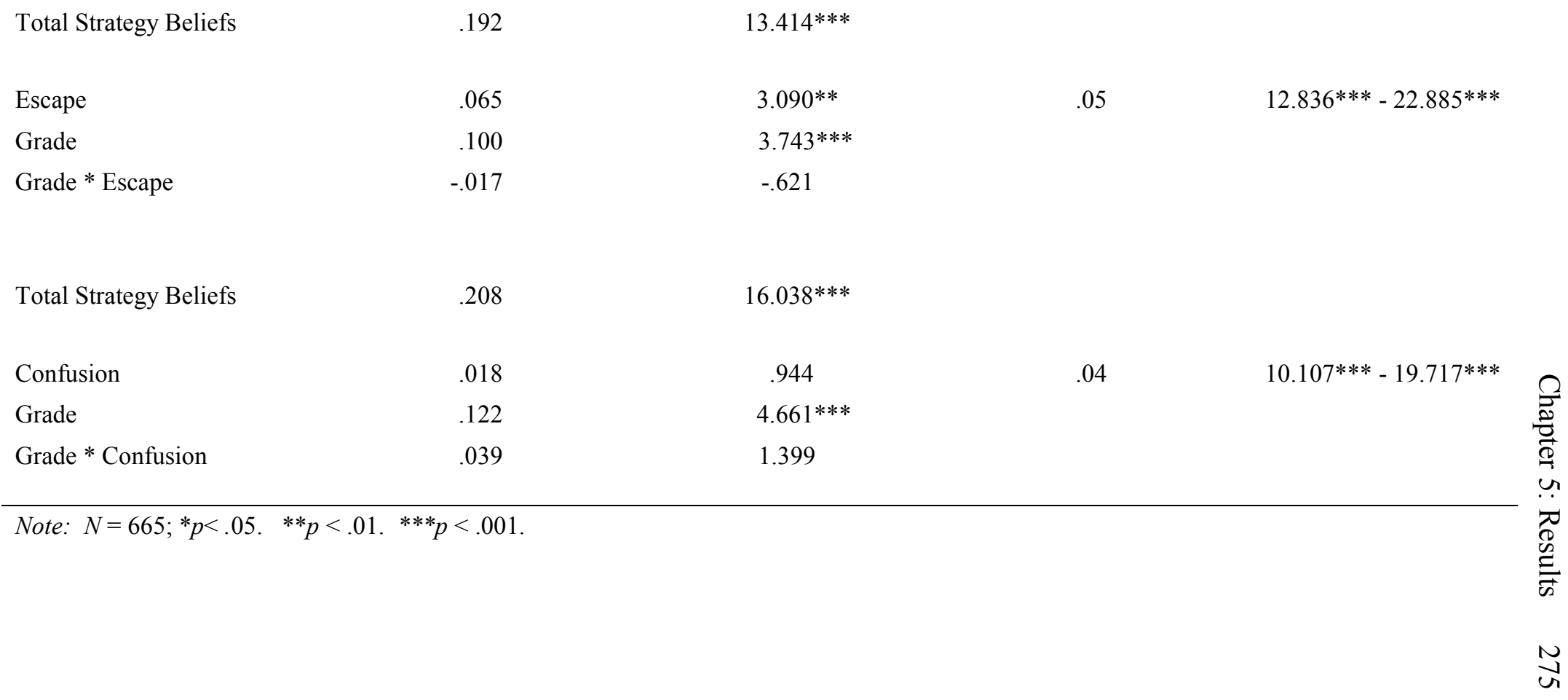


Table 51

Age Differences in the Feedback Effects of Ways of Coping on Changes in Total Capacity Beliefs - Mastery Coping

\begin{tabular}{lllll}
\hline Predictor (fall) & $\beta$ & $\mathrm{t}$ & $\Delta \mathrm{R}^{2}$ & F-Range \\
\hline
\end{tabular}

Total Capacity Beliefs (spring)

\begin{tabular}{|c|c|c|c|c|}
\hline Total Capacity Beliefs & .317 & $17.648 * * *$ & & \\
\hline Problem-Solving & -.016 & -.646 & .02 & $4.552 * *-10.459 * *$ \\
\hline Grade & -.127 & $-4.096 * * *$ & & \\
\hline Grade * Problem-Solving & .016 & .458 & & \\
\hline Total Capacity Beliefs & .310 & $15.887 * * *$ & .02 & $5.018^{* *}-10.553^{* *}$ \\
\hline Information-Seeking & -.007 & -.298 & & \\
\hline Grade & -.128 & $-4.114^{* * *}$ & & \\
\hline Grade * Info-Seeking & .032 & 1.030 & & \\
\hline \multicolumn{5}{|c|}{ Note: $N=665 ; * p<.05 . \quad{ }^{* *} p<.01 .{ }^{* * *} p<.001$. } \\
\hline
\end{tabular}


Table 52

Age Differences in the Feedback Effects of Ways of Coping on Changes in Total Capacity Beliefs - Helplessness Coping

\begin{tabular}{lllll}
\hline Predictor (fall) & $\beta$ & $\mathrm{t}$ & $\Delta \mathrm{R}^{2}$ & F-Range \\
\hline
\end{tabular}

Total Capacity Beliefs (spring)

\begin{tabular}{|c|c|c|c|c|}
\hline Total Capacity Beliefs & .285 & $13.137 * * *$ & & \\
\hline Escape & -.041 & -1.470 & .02 & $5.081 *-13.315 * * *$ \\
\hline Grade & -.124 & $-3.979 * * *$ & & \\
\hline Grade * Escape & -.014 & -.391 & & \\
\hline Total Capacity Beliefs & .292 & $16.233 * * *$ & & \\
\hline Confusion & -.047 & $-2.030^{*}$ & .03 & $7.683 * * *-6.101 * * *$ \\
\hline Grade & -.133 & $-4.291 * * *$ & & \\
\hline Grade * Confusion & -.021 & -.625 & & \\
\hline
\end{tabular}


Table 53

Age Differences in the Feedback Effects of Ways of Coping on Changes in Total Teacher Support - Mastery Coping

\begin{tabular}{lllll} 
Predictor (fall) & $\beta$ & $\mathrm{t}$ & $\Delta \mathrm{R}^{2}$ & F-Range \\
\hline
\end{tabular}

Total Teacher Support (spring)

\begin{tabular}{|c|c|c|c|c|}
\hline Total Teacher Support & .316 & $29.354 * * *$ & \multirow{4}{*}{.01} & \multirow{4}{*}{$3.297 *-8.271^{* * *}$} \\
\hline Problem-Solving & .007 & .453 & & \\
\hline Grade & .066 & $3.047 * *$ & & \\
\hline Grade * Problem-Solving & .031 & 1.358 & & \\
\hline Total Teacher Support & .313 & $29.015^{* * *}$ & \multirow{4}{*}{.01} & \multirow{4}{*}{$4.728^{* *}-12.380^{* * *}$} \\
\hline Information-Seeking & .015 & .909 & & \\
\hline Grade & .068 & $3.137^{* *}$ & & \\
\hline Grade * Info-Seeking & .036 & 1.556 & & \\
\hline \multicolumn{5}{|c|}{ Note: $N=665 ;{ }^{*} p<.05 . \quad{ }^{* *} p<.01 . \quad * * * p<.001}$. \\
\hline
\end{tabular}


Table 54

Age Differences in the Feedback Effects of Ways of Coping on Changes in Total Teacher Support - Helplessness Coping

\begin{tabular}{lllll}
\hline Predictor (fall) & $\beta$ & $\mathrm{t}$ & $\Delta \mathrm{R}^{2}$ & F-Range \\
\hline
\end{tabular}

Total Teacher Support (spring)

\begin{tabular}{|c|c|c|c|c|}
\hline Total Teacher Support & .310 & $28.334 * * *$ & & \\
\hline Escape & -.027 & -1.670 & .01 & $4.445^{* *}-10.527^{* * *}$ \\
\hline Grade & .073 & $3.356^{* *}$ & & \\
\hline Grade * Escape & -.015 & -.664 & & \\
\hline Total Teacher Support & .316 & $29.306 * * *$ & & \\
\hline Confusion & -.023 & -1.421 & .01 & $3.165^{*}-9.568^{* * *}$ \\
\hline Grade & .065 & $3.004 * *$ & & \\
\hline Grade * Confusion & -.006 & -.250 & & \\
\hline
\end{tabular}


Table 55

Age Differences in the Feedback Effects of Engagement on Changes in Perceived Control and Total Teacher Support

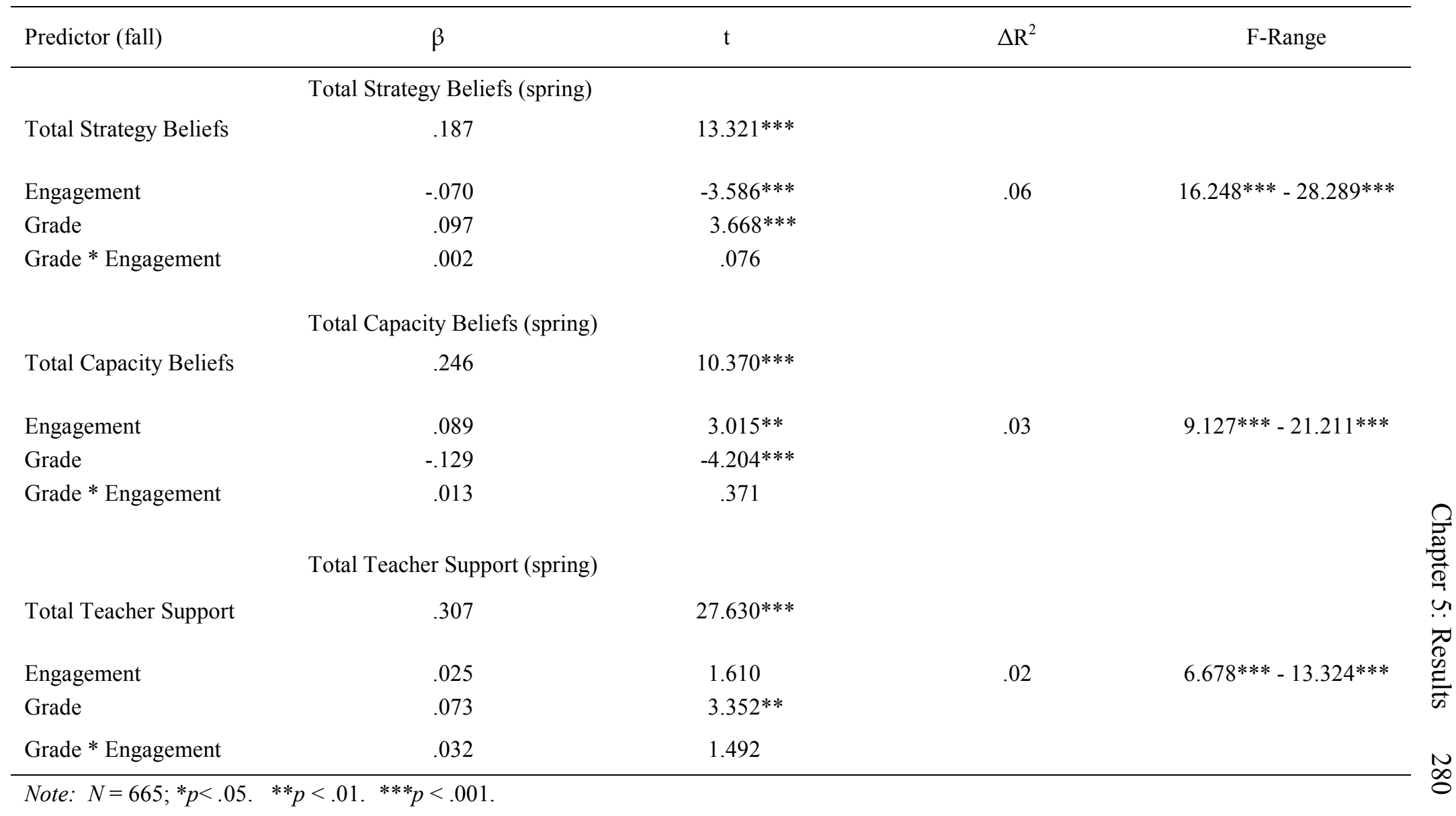




\section{CHAPTER 6: DISCUSSION}

\section{Summary of Findings}

Study overview. Developmental patterns of perceived control and coping in school-aged children are the subject of the present study; perceived control and coping are conceptualized as internal personal resources that develop over time and which are useful in many different contexts when children are faced with challenging events or setbacks (Compas, 1987; Greene, 2007; Wigfield \& Eccles, 2002; Wolchik \& Sandler, 1997). Perceived control is a tri-partite construct encompassing what one believes about one's ability to influence positive outcomes and avoid negative ones, with beliefs about the strategies that lead to success, as well as one's capacity to enact those strategies, at the heart of the construct (Skinner, 1998). Coping is conceptualized according to a framework of families of functionally similar but structurally distinct strategies employed in stressful circumstances (Skinner, Edge, Altman, \& Sherwood, 2003).

The classroom is one such context where the influence of perceived control and coping on children's outcomes may be observed. At school, children are faced with difficult academic tasks every day that can threaten levels of engagement and subsequent overall achievement. What they believe about themselves in relation to those challenges and the strategies they employ to manage them are instrumental in determining whether they stay engaged or become disaffected, and ultimately perform at levels of achievement that are beneficial to their long-term educational pursuits. 
Engagement as a multi-faceted construct is used in this study as a proximal outcome, positively related to the more distal outcome of overall achievement (Fredericks, et al., 2004; Skinner, Furrer, Marchand, \& Kindermann, 2008; Skinner, Kindermann, \& Furrer, 2009; Skinner et al., 2009; Wigfield et al., 2015).

The classroom teacher as a possible social context that can facilitate or hinder the accrual of motivational resources has been investigated by a number of researchers and has been included in this study as a social antecedent to these developmental processes (Connell, Spencer \& Aber, 1994; Marchand \& Skinner, 2007; Oettingen, Little, Lindenberger, \& Baltes, 1994; Schneewind, 1995). The type and strength of support provided by the teacher may have an effect on the development of children's internal resources.

Underlying the relationships hypothesized by this study is a motivational framework derived from action-theoretic concepts and Self-Determination Theory (Deci \& Ryan, 1985), synthesized as the Self-Systems Model of Motivational Development (SSMMD), in which competence is proposed as one of three fundamental psychological needs that must be met in order for motivated action to proceed (Connell, 1990; Connell \& Wellborn, 1991; Skinner \& Wellborn, 1994). Perceived control is commonly used as the proxy measure for the health of the competence system. The SSMMD was used by this researcher in her unpublished Master's thesis study to specify four individual process models, one for each of four different ways of coping, that describe how these constructs relate to one another. The 
present study extends this work and makes a contribution to the existing body of literature by examining the relationships illustrated in those models to determine how and to what extent perceived control and coping change differentially with age. Finally, feedforward and feedback cycles were examined to understand how these relationships influence and shape one another iteratively over time.

Study Goals. The first goal of this study was to investigate the normative agerelated differences in the constructs of the model through an examination and comparison within and between grades of the mean-levels of the constructs, concurrently and across time points. Additional analyses under this goal focused on evaluating progressions in the amount of change occurring in those mean levels from fall to spring.

The second goal was to explore how the relationships between these constructs change as normative development proceeds. The structural model previously identified by this researcher describing these relationships was tested for age differences through a series of analyses testing hypotheses accounting for each pathway in the model, including investigation of questions related to feedback and feedforward effects that may be operating as development proceeds. Hypotheses referencing possible mediated pathways were also tested.

Normative age-related analyses took the form of mean-level comparisons via t-test procedures, correlational comparisons, and regression analyses to determine the significance of face-value differences in correlations and amounts of change over 
time. Hypothesis testing for the structural model was accomplished through multiple regression procedures that included an interaction term, with grade as the moderating factor. The majority of results were non-significant; however, some demonstrated near significance in the $\mathrm{p}$-value and were nonetheless illustrative of the developmental trends at work. The discussion that follows here will highlight these trends, in spite of the lack of statistically significant differences.

Study findings are organized around the two major goals, with mean-level differences presented first, followed by results of the hypothesis testing, organized around each of the four primary study research questions.

\section{Profiles of Normative Development}

This study examined the between- and within-group differences in the mean levels of the primary study constructs of perceived control, coping, and engagement. Examination of between-grade changes in mean levels was conducted in two ways: 1) through comparison of means at concurrent time points, and 2) through comparison of the amount of change occurring in each grade from fall to spring. Examination of within-grade change was examined through comparison of mean-levels from fall to spring.

Perceived control. Based on the review of the literature presented earlier, it was expected that a shift would be seen in control beliefs from overestimation by younger children of their capacity to effectively employ successful strategies to their advantage, to a more realistic assessment as children get older of the uncontrollable 
nature of external strategies. This shift would be reflected in the decline in strength of capacity beliefs and the increase in the strength of uncontrollable strategy beliefs over time. Overall, a decline in perceived control was expected to be evident for older children as they approach the transition to middle school.

Figures 24-29 depict comparison graphs for each component of each of the study target constructs. For all graphs presented in this section, the following conventions are used to illustrate important findings: significant differences between grades at concurrent time points are indicated by a box that spans the bottom of the two bars that depict significantly different results, with notation indicating at what level the difference between the grades on that measure is significant (i.e., ${ }^{*} p<.05$, $\left.{ }^{* *} p<.01,{ }^{* *} p<.001\right)$. A broken line (red) that connects the fall and spring bars for a grade on any particular measure indicates a significant within-grade change in that measure from fall to spring. To indicate where the magnitude of within-grade fall-tospring changes is significantly different between the grades, a solid line connecting the fall and spring bars is used rather than a broken line, and appears for the grade with the largest change.

As can be seen in Figures 24 and 25, the pattern of changes from $4^{\text {th }}$ grade to $6^{\text {th }}$ grade in capacity and strategy beliefs follows the same pattern as described by previous research (Skinner, Zimmer-Gembeck, \& Connell, 1998). Capacity beliefs start out high and remain such for younger children, with a trend towards the noted decline seen for older children. Older children's assessment of their capacity for all 


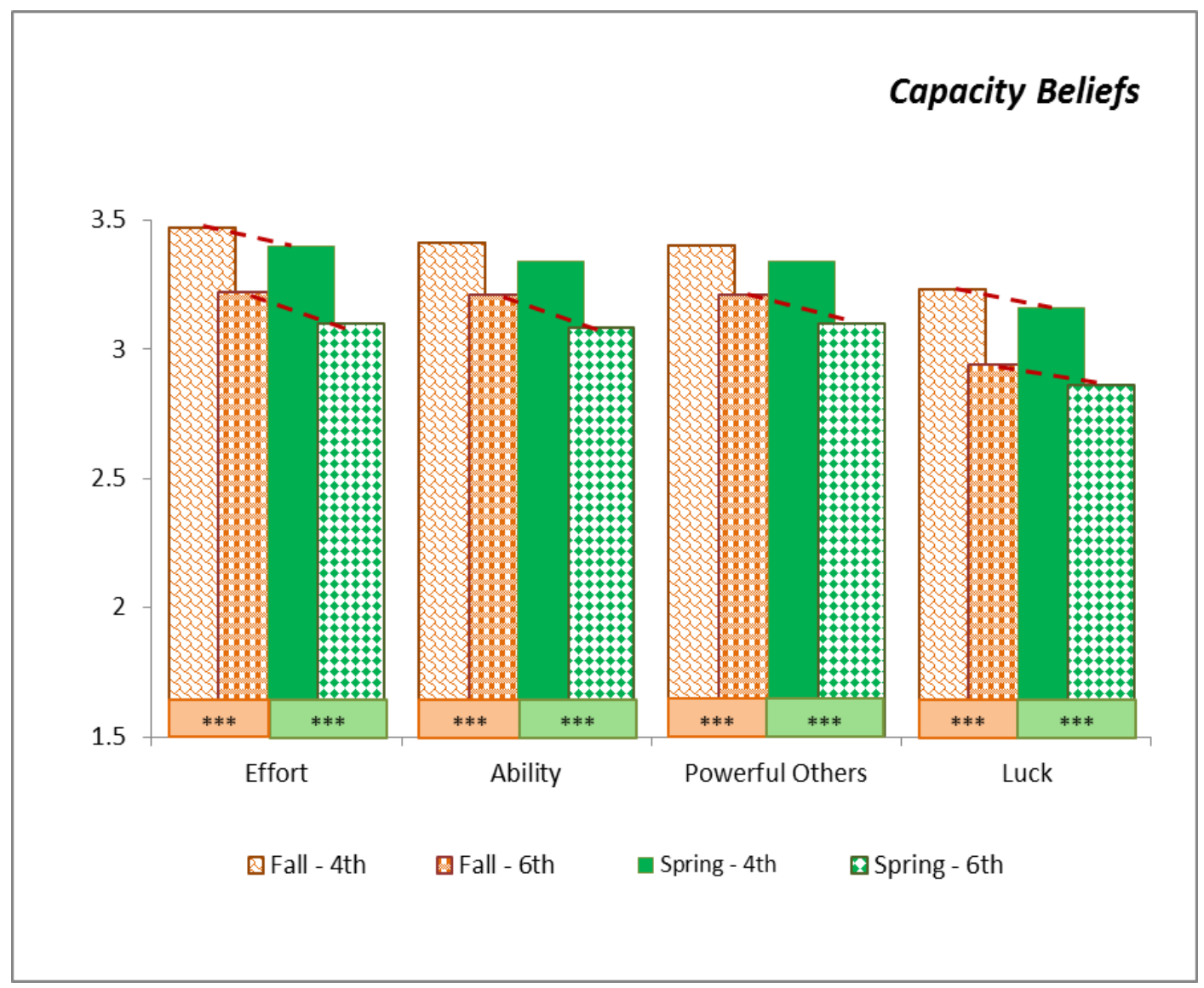

Figure 24: Capacity Beliefs comparison graph - between and within grade differences.

success strategies is substantially lower than that of younger children at both time points, suggesting that in all likelihood, the slight decline seen for the younger children continues steadily as children get older. At the same time, belief in effort as a means to success also starts out high for both grades, and remains stable for younger children, but shows a marked decline as children get older. Beliefs about ability remain flat, with little differentiation by age, while movement in opposite directions is seen for uncontrollable success strategies. Younger children hold firm in their belief in all strategies being useful to achieve desired ends, even the uncontrollable ones, 


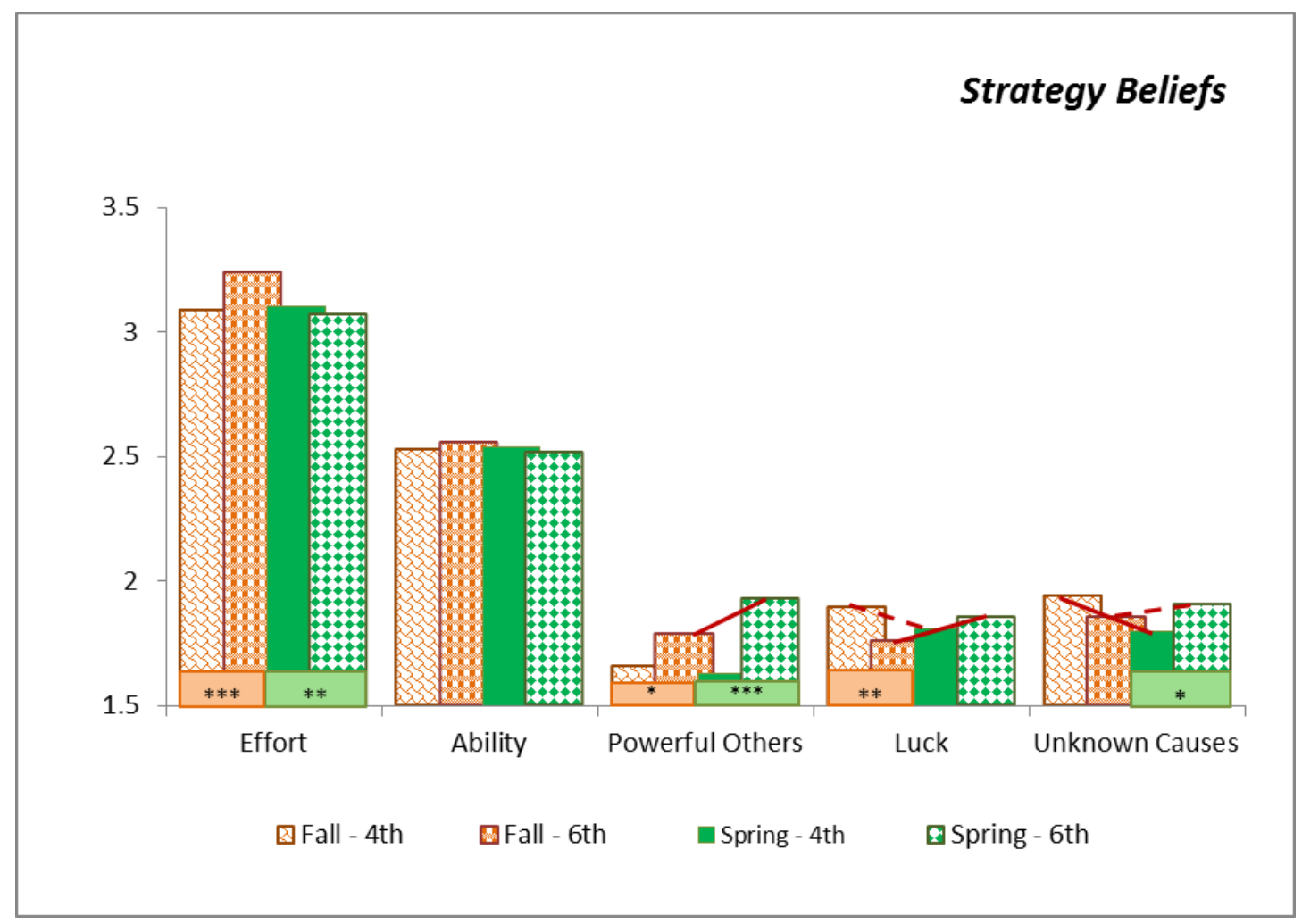

Figure 25: Strategy Beliefs comparison graph - between and within grade differences.

which is consistent with the expectation that younger children tend to overestimate the available contingencies in their environment and their capacity to successfully engage them. The older children, however, have begun to realize the limits of their own capacity to exert influence over uncontrollable strategies, and demonstrate increased belief that success may in fact be due to factors outside their control, which contributes to feelings of self-doubt and uncertainty about how to be successful that are seen at times of transition, particularly the transition to middle school.

The amount of change occurring for each grade is uniform for capacity beliefs, with no significant differences noted; however, for strategy beliefs, the amount of 
change occurring within-grade for uncontrollable strategies is greater for older children than for younger children, except for Unknown strategies, even though the changes occurring are significant across time for both grades. Table 56 displays the numerical changes in mean scores for the capacity and strategy beliefs components, with the larger change for each component highlighted where a significant difference in the magnitude of the change between the grades was found. Significance of the between-grade difference is noted on the left of the table; fall-to-spring within grade significance is noted in the change column on the right.

\begin{tabular}{|c|c|c|c|c|c|c|}
\hline \multicolumn{7}{|c|}{$\begin{array}{l}\text { Table } 56 \\
\text { Significant Differences in the Amount of Change in Fall to Spring Mean Scores for Strategy Beliefs }\end{array}$} \\
\hline \multirow{2}{*}{ Capacity Beliefs } & \multicolumn{2}{|c|}{4 th } & \multicolumn{2}{|c|}{6 th } & \multicolumn{2}{|c|}{ Change } \\
\hline & $\boldsymbol{F}$ & $s$ & $\boldsymbol{F}$ & $s$ & 4th & 6th \\
\hline Effort & 3.47 & 3.40 & 3.22 & 3.10 & $-.07^{* *}$ & $-.12 * * *$ \\
\hline Ability & 3.41 & 3.34 & 3.21 & 3.08 & -.07 & $-.13 * *$ \\
\hline Powerful Others & 3.40 & 3.34 & 3.21 & 3.10 & -.06 & $-.11 * *$ \\
\hline Luck & 3.23 & 3.16 & 2.94 & 2.86 & $-.07 *$ & $-.08 * *$ \\
\hline \multirow{2}{*}{ Strategy Beliefs } & \multicolumn{2}{|c|}{ 4th } & \multicolumn{2}{|c|}{6 th } & \multicolumn{2}{|c|}{ Change } \\
\hline & $F$ & $S$ & $F$ & $S$ & 4th & 6 th \\
\hline Effort & 3.09 & 3.10 & 3.24 & 3.07 & .01 & -.17 \\
\hline Ability & 2.53 & 2.54 & 2.56 & 2.52 & .01 & -.04 \\
\hline Powerful Others*** & 1.66 & 1.63 & 1.79 & 1.93 & -0.03 & $0.14 * * *$ \\
\hline $\operatorname{Luck}^{* * *}$ & 1.90 & 1.81 & 1.76 & 1.86 & $-0.09 *$ & $0.1^{* *}$ \\
\hline Unknown** & 1.94 & 1.8 & 1.86 & 1.91 & $-0.14 * *$ & $0.05 *$ \\
\hline
\end{tabular}

Note: $* p<.05 ; * * p<.01 ; * * * p<.001$.

Figure 26 depicts the grade comparison for generalized control along with Total Strategy and Total Capacity Beliefs, and is consistent with expectations based on previous research, as well as the results presented for the strategy and capacity beliefs comparisons that suggest normative decline in control over time. 


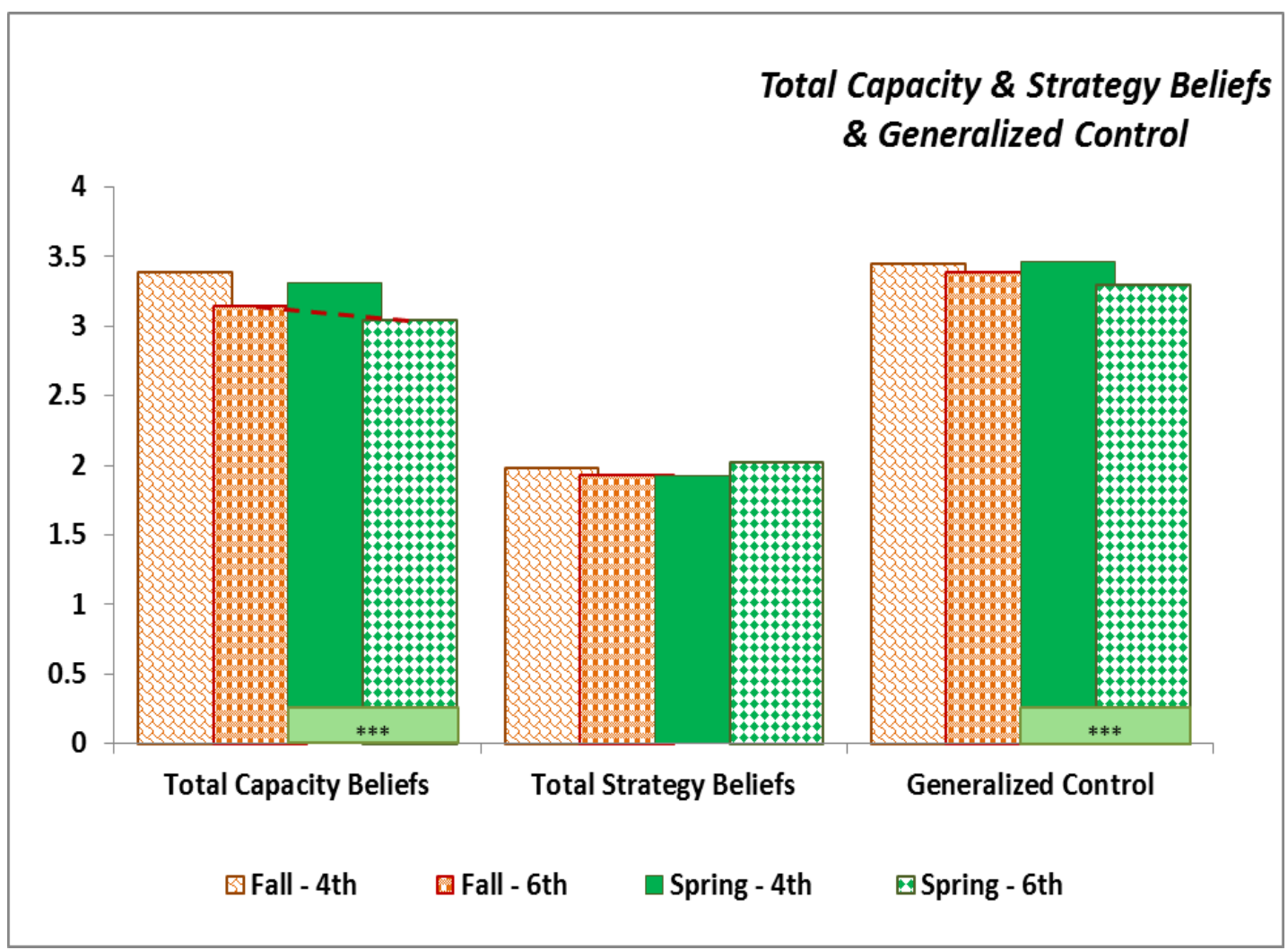

Figure 26: Generalized control beliefs comparison - between and within grade differences.

In sum, the results of these analyses for control suggest that the normative developmental pattern for perceived control is high capacity assessment and initial stability at younger ages, with subsequent decline over time as children get older. Children's expectations about the causes of success shift towards uncontrollable causes having more power than previously thought, and a more realistic view of the extent to which they have control over influencing uncontrollable causes in their favor. Belief in their ability to enact strategies that are inherently under their control (i.e., effort) is challenged in the face of this uncertainty and may suffer corresponding declines. 
Coping. Changes in coping have been noted in other studies to move from behaviorally based coping strategies at younger ages to cognitively based ones at older ages, corresponding with the expected development of cognitive capacities and the approach to formal operational thought (Compas et.al., 2001; Fields \& Prinz, 1997; Skinner \& Zimmer-Gembeck, 2007; Zimmer-Gembeck \& Skinner, 2011). Coping strategies children use tend to become more differentiated with age, particularly with respect to approach-type coping, while changes in avoidance-type coping are found to be relatively stable and constitute a simple exchange of behavioral avoidance for cognitive forms of avoidance. Overall, reliance on social partners is seen to decline with age, with social partner preference focused on adults for younger children, shifting for a brief time to peers as age progresses, and returning again to adults when children are older. Figure 27 presents the grade comparisons for each of the four ways of coping examined in the present study.

Consistent with expectations, coping in younger children is relatively stable across time, with a slight and non-significant decrease in Problem-Solving and a slight non-significant increase in Escape coping. Older children, on the other hand, demonstrate significant declines in both mastery ways of coping. This result is interesting, in that at first glance, it appears to run counter to the analysis of Fields and Prinz (1997), who found that children's use of instrumental and approach-type coping increased with age. However, in light of the integrated perspective offered by 


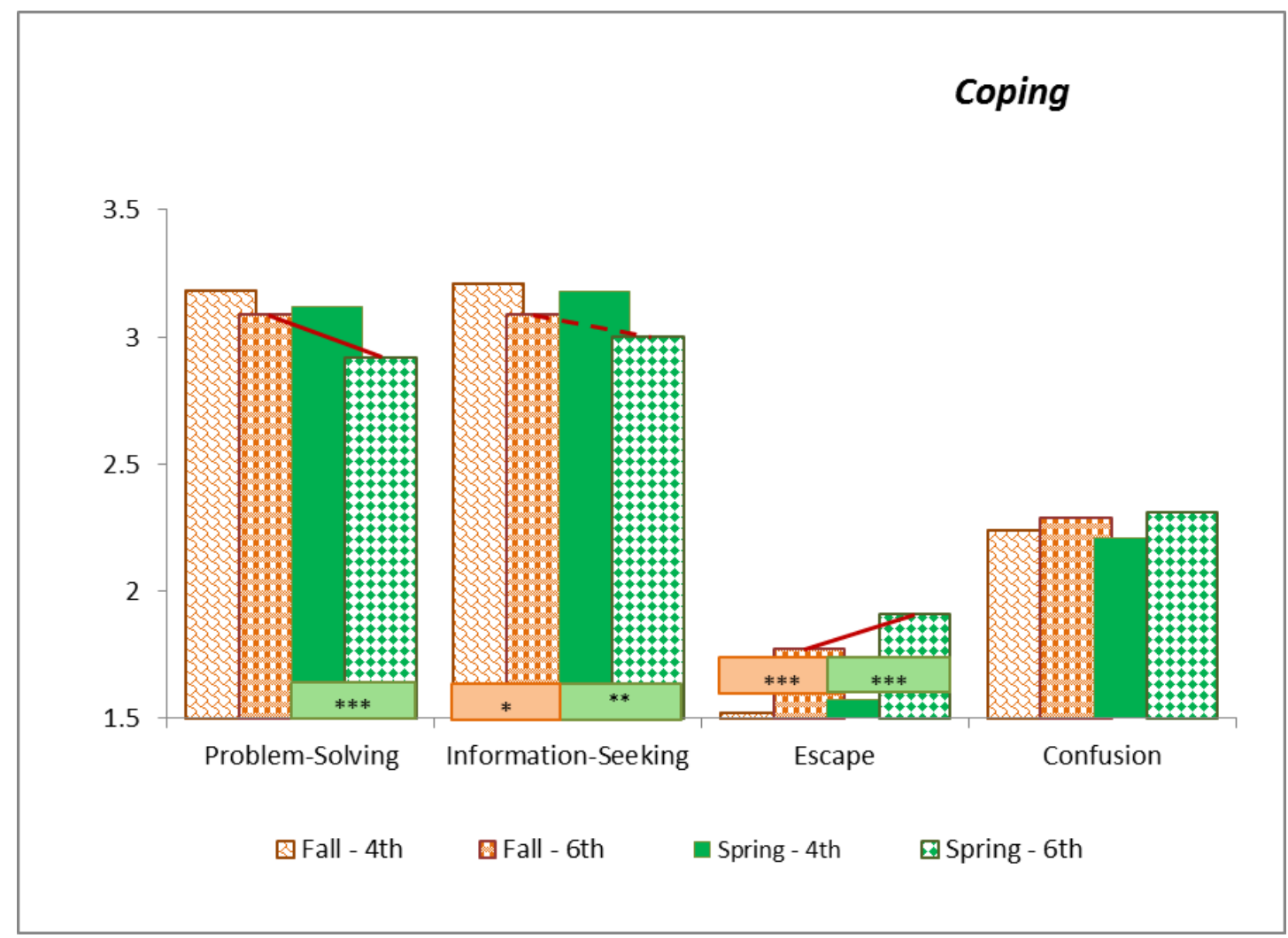

Figure 27: Ways of coping comparison graph - between and within grade differences.

Skinner and Zimmer-Gembeck (2007) that suggests children are better able to coordinate their efforts with the actions of others due to the emergence of metacognitive capacities, these results might be interpreted as just the beginning of that transition. Children in the sixth grade may be in a state of ambivalence in their use of mastery coping as they begin to shift their social preference for instrumental help back to adults, while at the same time experiencing a good deal of uncertainty about the amount of influence they have over productively engaging those they see as "powerful others" in their problem-solving and information-seeking efforts. Solid use of approach-type coping such as problem-solving and information-seeking may be more 
evident at grade levels higher than those examined in the present study. Alternatively, it is possible that the increased and differentiated use of instrumental approach-type strategies that is expected is being expressed through actions that are not captured by the measures used for mastery coping in this study, and the declines noted for the sixth grade students are indicative of the decline of behavioral-based strategies.

At the same time, a significant increase is noted for Escape coping. This pattern is consistent with the expectation of increased avoidance-type coping as children get older. Confusion for older children trends upwards, but the changes are not significant; nonetheless, this may be a signal of the expected shift from behaviorally-based to cognitive-based avoidance-type coping reported in previous research.

The magnitude of change from fall to spring was significantly different between the grades for Problem-Solving and Escape, with $6^{\text {th }}$ grade students experiencing larger declines compared to the slight declines of the $4^{\text {th }}$ grade students. Table 57 displays the numerical change in mean levels for those ways of coping where the magnitude of the fall-to-spring change was significantly different, with the larger change for each component highlighted where a significant difference in the magnitude of the change between the grades was found. Significance of the betweengrade difference is noted on the left of the table; fall-to-spring within grade significance is noted in the change column on the right. 


\begin{tabular}{|c|c|c|c|c|c|c|}
\hline \multicolumn{7}{|l|}{$\begin{array}{l}\text { Table } 57 \\
\text { Significant Difference } \\
\text { Measures }\end{array}$} \\
\hline \multirow{2}{*}{ Mastery Coping } & \multicolumn{2}{|c|}{4 th } & \multicolumn{2}{|c|}{6 th } & \multicolumn{2}{|c|}{ Change } \\
\hline & $\boldsymbol{F}$ & $s$ & $\boldsymbol{F}$ & $s$ & 4th & 6th \\
\hline Problem-Solving* & 3.18 & 3.12 & 3.09 & 2.92 & -0.06 & $-0.17 * * *$ \\
\hline Information-Seeking & 3.21 & 3.18 & 3.09 & 3.00 & -.03 & $-.09 * *$ \\
\hline Escape* & 1.52 & 1.57 & 1.77 & 1.91 & 0.05 & $0.14 * * *$ \\
\hline Confusion & 2.24 & 2.21 & 2.29 & 2.31 & -.03 & .02 \\
\hline
\end{tabular}

Note: $* p<.05 ; * * p<.01 ; * * * p<.001$.

Engagement. Engagement has been confirmed to be fairly high when children are young, showing a steady decline over time (Eccles \& Midgely, 1989; Eccles, et al., 1993; Skinner et.al., 1998). Figure 28 displays the comparison graphs for the components of Engagement examined in the present study.

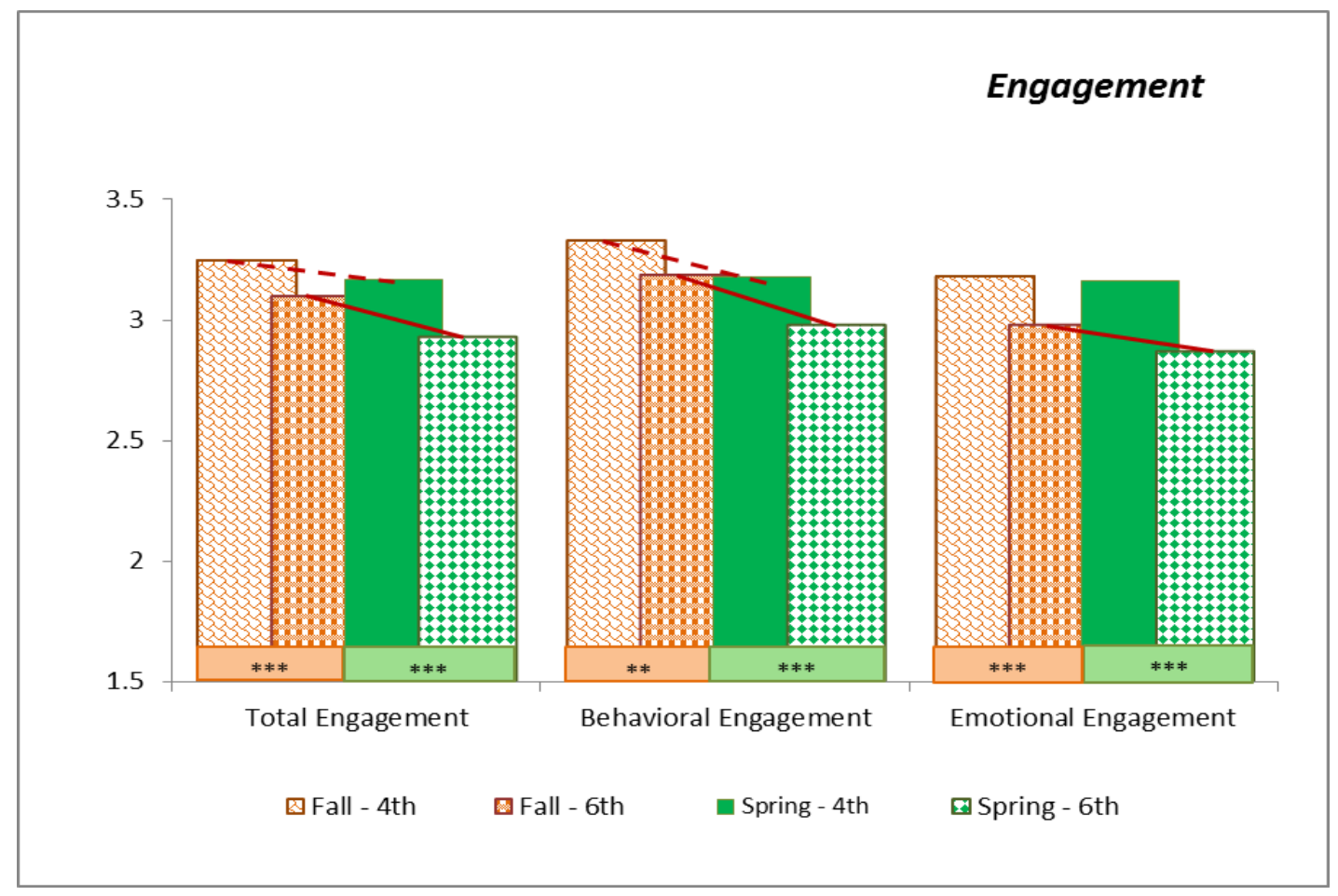

Figure 28: Engagement comparison graph - between and within grade differences. 
The expected declines are seen, slight but steady for the younger children, and sharper for the older children. Both grades experience a significant and sharper decline in Behavioral Engagement than in Emotional Engagement, and the mean differences between the grades are significant for all measures at both time points. The magnitude of the changes from fall to spring is also significant between the grades on all measures, with $6^{\text {th }}$ grade students showing larger overall declines than $4^{\text {th }}$ grade students. Table 58 displays the numerical change in the mean level for all Engagement measures, with the larger change for each component highlighted where a significant difference in the magnitude of the change between the grades was found. Significance of the between-grade difference is noted on the left of the table; fall-tospring within grade significance is noted in the change column on the right.

\begin{tabular}{|c|c|c|c|c|c|c|}
\hline \multicolumn{7}{|c|}{$\begin{array}{l}\text { Table } 58 \\
\text { Significant Differences in the Amount of Change in Fall to Spring Mean Scores for Engagement }\end{array}$} \\
\hline \multirow{2}{*}{ Engagement } & \multicolumn{2}{|c|}{$4 t h$} & \multicolumn{2}{|c|}{6 th } & \multicolumn{2}{|c|}{ Change } \\
\hline & $\boldsymbol{F}$ & $s$ & $\boldsymbol{F}$ & $s$ & 4th & 6 th \\
\hline Total Engagement*** & 3.25 & 3.17 & 3.1 & 2.93 & $-0.08 * * *$ & $-0.17 * * *$ \\
\hline Behavioral** & 3.33 & 3.18 & 3.19 & 2.98 & $-0.15 * * *$ & $-0.21 * * *$ \\
\hline Emotional ** & 3.18 & 3.16 & 2.98 & 2.87 & -0.02 & $-0.11 * * *$ \\
\hline
\end{tabular}

Note: $* p<.05 ; * * p<.01 ; * * * p<.001$.

Teacher Support. As a feature of the context that shapes children's perceptions of control and may influence the way they approach challenges (Connell, 1990; Furrer \& Skinner, 2003; Marchand \& Skinner, 2007), the discussion of normative development would not be complete without a description of the levels and changes in teacher support provided to children as the normative developmental trends just 
described unfold. Figure 29 displays the shifts in the components of teacher support across the year.

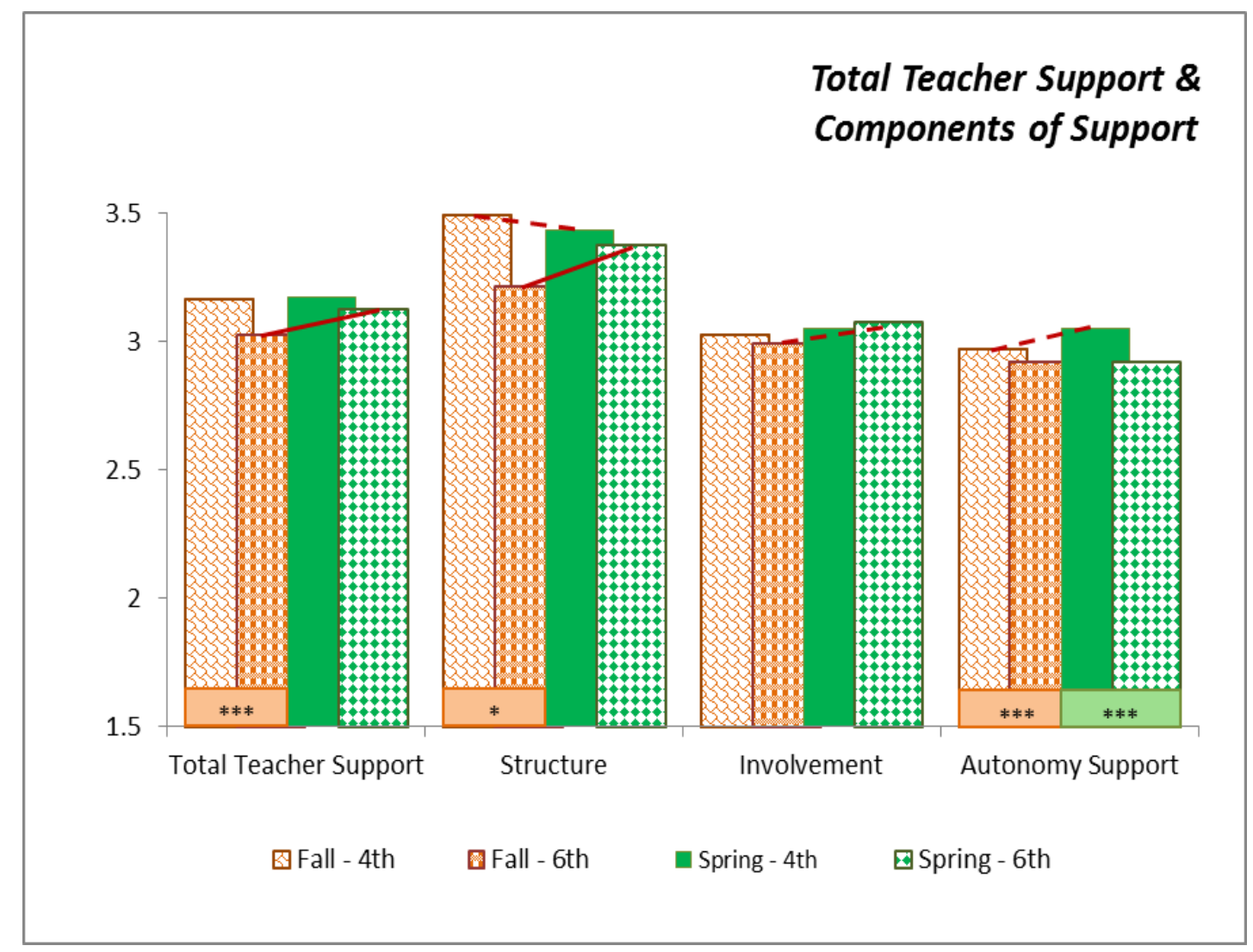

Figure 29: Teacher Support comparison graph - between and within grade differences.

Overall teacher support is higher for $4^{\text {th }}$ grade students than for $6^{\text {th }}$ grade students. Autonomy support and involvement show increases over the year, with no difference in the magnitude of the change between the grades. The biggest difference in how teachers interact with their students is seen in the provision of structure. As the year progresses, teachers tend to provide less structure to younger students, but more structure to older students. The net effect is that older students are experiencing 
increases in support from their teachers in all areas, while younger students are experiencing increases in support for autonomy and involvement, but decreases in provision of structure. Younger children may require more support for emerging autonomy needs as teachers work to foster a greater sense of responsibility in children, preparing them to move to the next grade with its increased expectations. In view of the decline seen in engagement for the older children, additional structure may be the teachers' way of drawing them back in and helping them regain their focus on academic tasks. Table 59 displays the numerical change in the mean level for all Teacher Support measures, with the larger change for each component highlighted where a significant difference in the magnitude of the change between the grades was found. Significance of the between-grade difference is noted on the left of the table; fall-to-spring within grade significance is noted in the change column on the right.

\begin{tabular}{|c|c|c|c|c|c|c|}
\hline \multicolumn{7}{|c|}{$\begin{array}{l}\text { Table } 59 \\
\text { Significant Differences in the Amount of Change in Fall to Spring Mean Scores for Teacher } \\
\text { Support }\end{array}$} \\
\hline \multirow{2}{*}{ Support Measure } & \multicolumn{2}{|c|}{4 th } & \multicolumn{2}{|c|}{6 th } & \multicolumn{2}{|c|}{ Change } \\
\hline & $\boldsymbol{F}$ & $s$ & $\boldsymbol{F}$ & $s$ & 4th & 6 th \\
\hline Total Teacher Support ${ }^{* * *}$ & 3.16 & 3.17 & 3.02 & 3.12 & .01 & $0.10 * * *$ \\
\hline Structure ${ }^{* * *}$ & 3.49 & 3.43 & 3.21 & 3.37 & $-0.06 * * *$ & $0.16 * * *$ \\
\hline Involvement & 3.02 & 3.05 & 2.99 & 3.07 & 0.03 & $0.08^{* * *}$ \\
\hline Autonomy Support & 2.97 & 3.05 & 2.87 & 2.92 & $0.08^{* * *}$ & 0.05 \\
\hline
\end{tabular}
Note: ${ }^{*} p<.05 ; * * p<.01 ; * * * p<.001$.

Summary of Normative Development. To summarize the results of analyses aimed at describing the normative progression of development of perceived control, coping, and engagement, this discussion considers first the summative profile of the 
younger children, then the older children, and then considers the larger picture of development in light of both.

Younger students, in general, exhibit a strong sense of generalized control over interactions with academic tasks, have strong and relatively stable beliefs about their capacity to control their outcomes, and are less certain about the effectiveness of external strategies for control than internal ones. They are highly engaged both emotionally and behaviorally, are strong and consistent in their use of mastery coping, and are not prone to using Escape coping, although they do exhibit somewhat higher Confusion coping. Over time, these levels do evidence slight declines, with more significant declines noted in their beliefs about their capacity for Effort and Luck, beliefs about the effectiveness of the uncontrollable strategy of Luck, and their overall Engagement, due to significant declines in Behavioral Engagement. Also, their understanding of what leads to success becomes more defined, as evidenced by significant declines in Strategy Beliefs for Unknown causes. Their teachers are very supportive, providing higher levels of autonomy and involvement, while backing off on provision of structure.

Older children are not nearly as strong in their Capacity Beliefs, which continue to decline significantly over the year. Their beliefs about effective strategies are fairly stable with respect to controllable strategies, but reflect a growing uncertainty about the controllability of success in the significant increase in the strength of beliefs about uncontrollable strategies, which in turn may diminish the 
confidence they have about the effectiveness of effort as a strategy for success. These changing beliefs about control are accompanied by a significant decrease in the use of mastery ways of coping, and a significant increase in avoidance coping in the form of Escape. Engagement also is in significant decline, both behaviorally and emotionally. Teachers of older children tend to provide lower levels of support at the beginning of the year, increasing all types of support over time. The biggest increase in teacher support is in the provision of structure.

The declining trend of the younger children, which in most cases represents just a trend and not a significant difference over the time period of a single year as measured by this study, might be characterized as the beginning of an overall trend that becomes more pronounced over time, with much sharper declines noted as children approach the transition to middle school. This view is supported by a simple comparison of the spring mean levels for the younger children and the fall mean levels for the older children. This comparison shows the end of the year trend for younger children as higher than the beginning levels for the older children on the expected measures of Capacity Beliefs, mastery ways of coping, Confusion coping, Engagement, and the external control strategies of Luck and Unknown causes. From this comparison, one can surmise that the trend noted for $4^{\text {th }}$ grade students continues over the next year and on into $6^{\text {th }}$ grade, providing a more continuous picture of the progression of development of these constructs. 


\section{Developmental Differences in the Process Relationships of the Structural Model}

Hypotheses overview. The second goal of this study was to examine the relationships described by the process model for developmental differences. This was accomplished through a series of analyses testing hypotheses about these relationships, based on the work conducted for this researcher's unpublished Master's thesis. This section provides first an overview of those hypotheses and the status of support obtained from the analyses, followed by a summary of the results, organized around the four research questions of the study, and a discussion of selected findings that are of particular interest.

The first research question asked about age differences in the feedforward and feedback relationships between coping and engagement and coping and academic achievement, and the mediational effect of engagement on the relationship between coping and achievement. It was expected that the relationships between coping and engagement would show age differences for all ways of coping, in both feedforward and feedback pathways, with older children exhibiting stronger effects than younger children for all ways of coping except Escape. Significant age differences in the prediction of changes in Engagement by ways of coping were expected only for helplessness coping, with effects stronger for older children for Confusion, and stronger for younger children for Escape. The pathway between coping and academic achievement was expected to evidence age differences only for Information-Seeking and Escape, with older children having stronger effects for Information-Seeking and 
younger children showing greater effects for Escape, and age differences in feedback effects of achievement on coping expected only for Escape, with effects stronger for younger children. Finally, no age differences were expected in the mediational effect of Engagement on the relationship between coping and achievement. Results did not support any of the hypotheses describing individual pathways, and only partially supported the mediational hypotheses, in that while no age differences were found for any of the eight models tested (one for each of four ways of coping at two time points), only partial mediation could be confirmed rather than full mediation.

The second research question looked for age differences in the relationship between the aggregate components of control and ways of coping, and the maximum control aggregate (CONMAX) and changes in coping. It was expected that age differences would be detected for the effect of Total Capacity beliefs on all ways of coping, with effects stronger for older children for Information-Seeking and Confusion, and stronger for younger children for Problem-Solving and Escape. Age differences in the effects of Total Strategy beliefs on coping were expected only for Confusion coping, with effects stronger for older children. Age differences in the relationship of maximum control and changes in coping were expected only for the pathway to Escape, with effects stronger for younger children. Although a significant age difference was detected for Total Strategy beliefs, examination of the disaggregated component measures did not yield any age differences for a specific strategy measure. Thus, results did not support any of these specific hypotheses. 
Differential impact of support from the social context on coping efforts, as well as changes in coping across the year, was the focus of the third research question. A significant age difference was expected for the relationship between the aggregate measures of teacher support and all ways of coping. The effect was expected to be stronger for older children for all ways of coping except problem-solving, which was expected to be stronger for younger children. The impact of teacher support on changes in coping was not expected to show any significant differences by age. Results did not support any of the hypotheses regarding age differences in the impact of teacher support on ways of coping. In fact, teacher support was not significantly related to any way of coping except Escape, and no age differences were found. Also, teacher support was not related to changes in any way of coping, including Escape.

The fourth and last research question addressed the possibility of feedback effects of coping and engagement on perceived control and teacher support. It was expected that feedback effects would be significantly stronger as children got older with respect to both Coping and Engagement feeding back to shape changes in teacher support and perceived control, with one exception; the feedback pathway from Problem-Solving coping to changes in control was not expected to show significant age differences. Results did not support these hypotheses, as no significant age differences were found, and Problem-Solving coping was not related to changes in control. 
In short, very little support for the original study hypotheses regarding age differences resulted from the analyses that were conducted. This does not mean, however, that the analyses were in vain and produced no useful information. Even though most of the analyses did not yield a significant finding in terms of the statistical parameter of a p-value equal to or less than .05 , some did approach significance, and when the results were graphed, it was evident that the hypothesized direction and strength for some of the specific relationships was in fact confirmed. Graphing all results helped illustrate where there was support for the developmental trends outlined in the overall analysis of mean-level differences, and some of those graphs are presented in this section for selected analyses. All results graphs for this study, including those not presented in this discussion are available in Appendix C.

The next part of this section will discuss the findings for each research question from a developmental trends perspective, with relevant details displayed in Tables $60-64$, and further illustrated with results graphs when a finding of particular interest is presented. The tables show the pathways tested, whether the process link describing the construct relationships was confirmed by the present study, and for which of the four previously defined coping models it was confirmed. A simple indicator of which age group has a better outcome on the specified pathway is noted, and for which coping model(s). Stating which group has a better outcome is another way of depicting the hypothesized relationships. 
Results by Research Question

Research Question 1: Coping, Achievement, \& Engagement. Previous research supports a strong connection between levels of engagement and subsequent achievement, and identifies coping as a factor that may influence the development of engagement (Compas, 1987; Wigfield \& Eccles, 2002; Wigfield et al., 2006; Wigfield et al., 2015; Wolchik \& Sandler, 1997). Feedforward and feedback relationships were tested, as well as models proposing engagement as a mediator between coping and achievement. Results illustrate the reciprocal relationship between coping and engagement, as well as provide partial support for a mediating role for engagement. Age differences in all effects were not supported at a statistically significant level; however, developmental trends in how these relationships work were noted. Table 60 summarizes these observations; any entry that is bolded indicates a result that was consistent with the direction of the originally hypothesized relationships. The first three pathways describe the reciprocal relationship between coping and engagement, and detail the component feedforward and feedback effects. The graphed results for these pathways are depicted in Figures $30-32$. The results are intriguing in that, when taken together, they clearly suggest how the feedback loop differs with age.

The link from mastery coping to engagement shows a stronger effect for older children, which is consistent with the originally hypothesized relationship, and in contrast to the result for the link from mastery coping to changes in engagement, which was stronger for younger children, suggests that early use of mastery coping is 


\begin{tabular}{|c|c|c|c|c|}
\hline $\begin{array}{l}\text { Table } 60 \\
\text { Structural Relationships and Developm }\end{array}$ & ental Trends for & Research Qu & & \\
\hline Model Pathway & $\begin{array}{c}\text { Consistent } \\
\text { with which } \\
\text { coping models }\end{array}$ & Trends sugg & $\begin{array}{l}\text { st things are } \\
\text { for.... }\end{array}$ & etting better \\
\hline $\begin{array}{c}\text { Research Question 1: } \\
\text { Coping, Engagement, \& Achievement }\end{array}$ & & $4^{\text {th }}$ Graders & $6^{\text {th }}$ Graders & $\begin{array}{c}\text { No } \\
\text { Differences }\end{array}$ \\
\hline Coping $\longrightarrow$ Engagement & ALL & CF & PS / IS & ES \\
\hline Coping $\longrightarrow \Delta$ Engagement & $\begin{array}{c}\text { NOT } \\
\text { CONFIRMED }\end{array}$ & PS / IS & ES & CF \\
\hline Engagement & ALL & IS /CF & PS & ES \\
\hline Coping $\longrightarrow$ Achievement & ES & & & $\begin{array}{l}\mathrm{PS} / \mathrm{IS} / \mathrm{ES} \\
\mathrm{CF}\end{array}$ \\
\hline Achievement $\longrightarrow$ & ES & PS / IS / ES & & $\mathrm{CF}$ \\
\hline Coping $\longrightarrow$ Engmnt $\longrightarrow$ Achvmnt & $\begin{array}{c}\text { PS / IS / CF } \\
\text { (fall) } \\
\text { PS / CF } \\
\text { (spring) }\end{array}$ & $\begin{array}{l}\text { All except } \\
\text { fall IS }\end{array}$ & & $\begin{array}{l}\text { IS } \\
\text { (fall) }\end{array}$ \\
\hline
\end{tabular}

more important in maintaining engagement for older children, whereas it is more important in building engagement for younger children. This observation reflects the strong endorsement of mastery coping by both groups seen in the mean levels of coping.

Note that Escape coping shows a lesser influence on changes in Engagement for $6^{\text {th }}$ graders than for $4^{\text {th }}$ graders, where, before removing the effects of early Engagement, there was no difference between the grades. When the effects of Engagement that include the changes over time feed back into coping efforts, the effect on Escape coping remains essentially the same for both grades. 


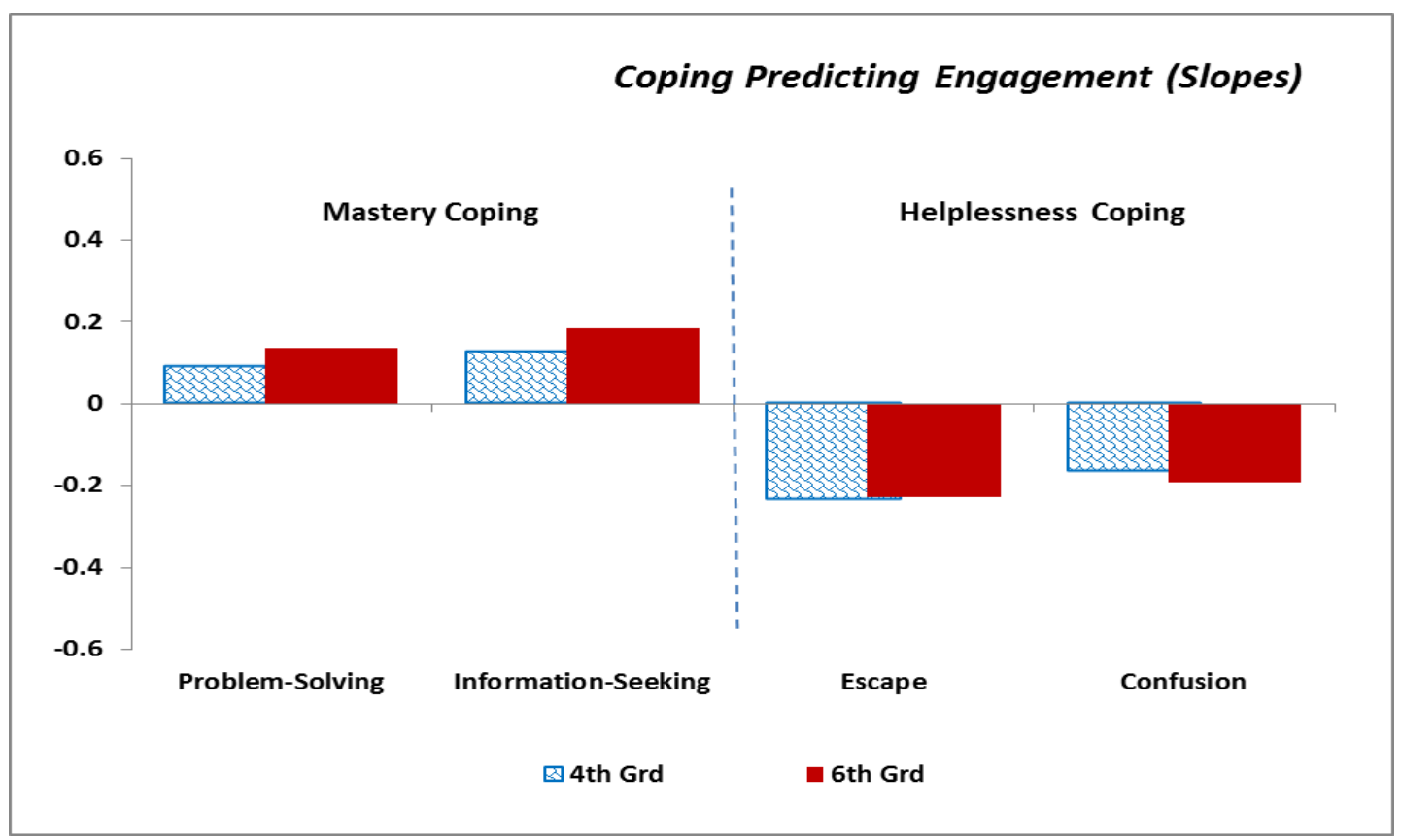

Figure 30: Coping predicting Engagement; $6^{\text {th }}$ graders doing better than $4^{\text {th }}$ on PS \& IS.

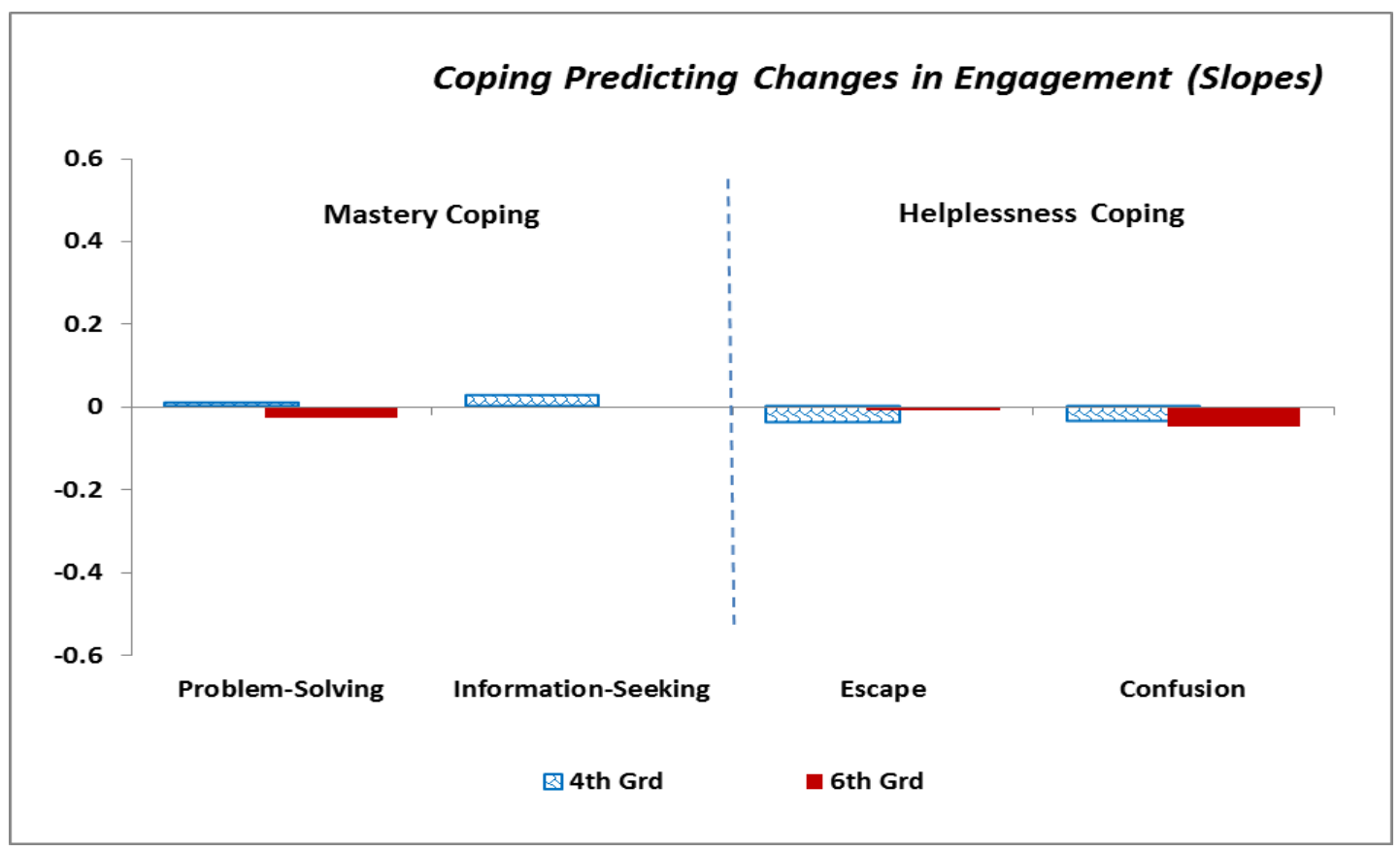

Figure 31: Coping predicting changes in Engagement; $4^{\text {th }}$ graders doing more poorly on Escape. 
Chapter 6: Discussion 306

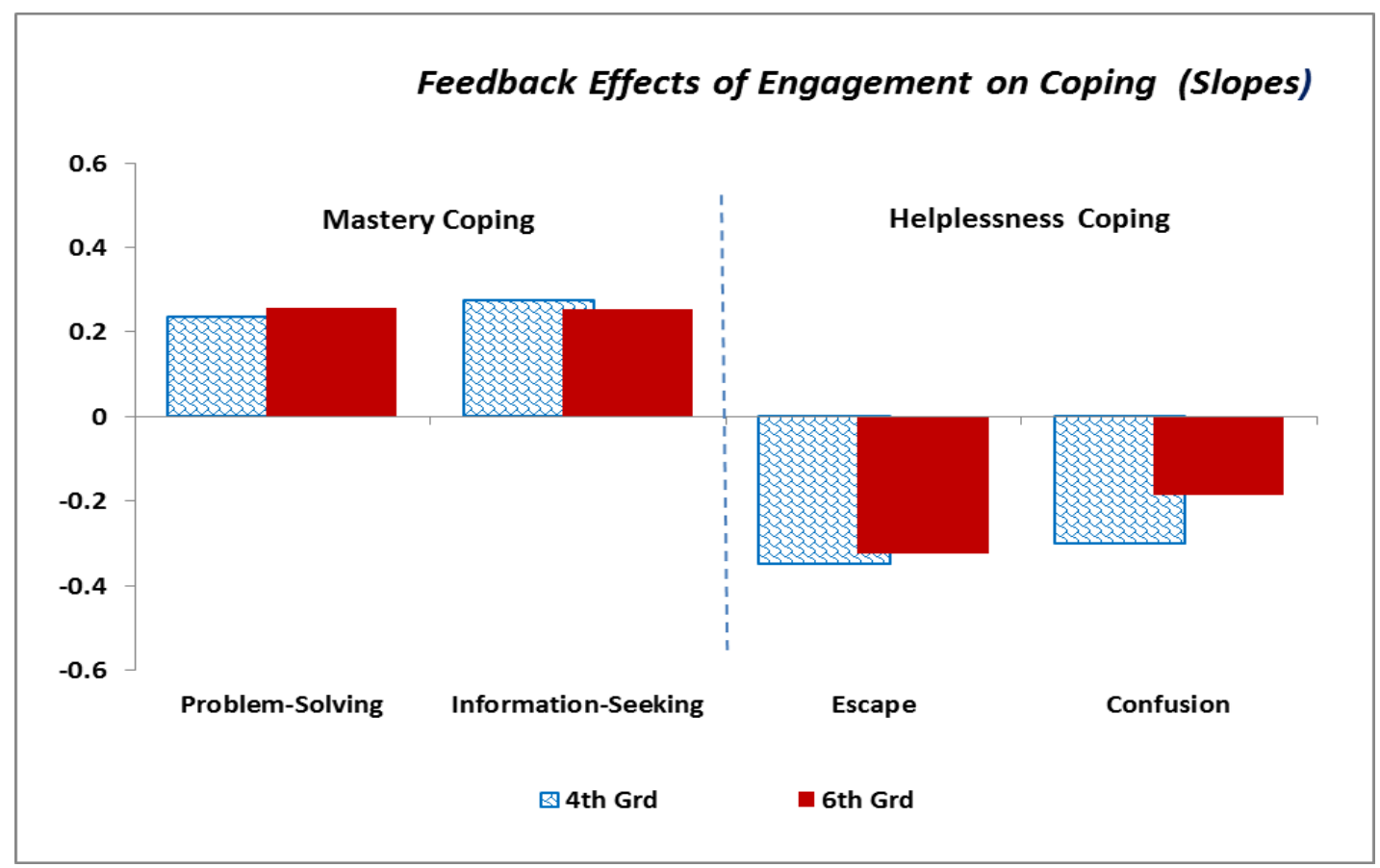

Figure 32: Feedback effects of Engagement on Coping; $4^{\text {th }}$ graders doing better than $6^{\text {th }}$ overall.

The result for changes in Engagement due to Escape coping helps to explain what these findings mean in terms of how the feedback loop operates. The feedforward effect of coping on Engagement suggests that regardless of age, the effect of Escape coping on later Engagement will be the same, as virtually no difference in the predicted slopes between the grades was noted. However, the effect of Escape coping on how Engagement changes over time is stronger for younger children than for older children, meaning more of the variance in Engagement for $4^{\text {th }}$ graders than $6^{\text {th }}$ graders is due to the effects of early coping; but the feedback effect of the drop in Engagement associated with early coping does not have as big an impact on subsequent coping for the younger children as it does for older children, as evidenced by the lack of any appreciable difference between the grades on this relationship. This 
may account for the lesser decline in Engagement noted for $4^{\text {th }}$ grade students reflected in the mean levels, as the mean levels for helplessness coping showed higher endorsement of Confusion coping rather than Escape coping for the $4^{\text {th }}$ graders, compared to stronger endorsement of Escape coping for the $6^{\text {th }}$ grade students.

Escape coping is the only coping model for which a significant effect of coping on achievement was found, as expected, and the only model for which Engagement was not a mediator of the effects of coping on achievement at either fall or spring time points. All mediator models favored younger children, except for InformationSeeking in the fall, which did not demonstrate any appreciable difference in outcomes between the two groups of children.

Research Question 2: Control predicting coping. The influence of the perceived control construct on academic outcomes has been well documented for children in middle childhood (Skinner, et al., 1998; Skinner \& Zimmer-Gembeck, 2011). The focus of this research question was to confirm the predictable pattern of its influence on coping, and examine that relationship for age differences to better understand how perceived control and coping develop in synergy with one another as normative development proceeds. The expectation is that as a child begins to differentiate effort from ability, the strength of the influence of component parts of the construct changes differentially, with substantive impacts on the development of coping resources. 
Observations for the results of these analyses are summarized in Table 61. While the process links for the overall models were largely confirmed, none of the hypothesized relationships that specify for which group the effects would be stronger were confirmed.

\begin{tabular}{|c|c|c|c|c|c|}
\hline \multicolumn{6}{|c|}{$\begin{array}{l}\text { Table } 61 \\
\text { Structural Relationships and Developmental Trends for Research Question } 2\end{array}$} \\
\hline Model Pathway & $\begin{array}{l}\text { Consistent } \\
\text { with which } \\
\text { coping models }\end{array}$ & \multicolumn{4}{|c|}{$\begin{array}{l}\text { Trends suggest things are getting } \\
\text { better for.... }\end{array}$} \\
\hline $\begin{array}{l}\text { Research Question } 2 \\
\text { Coping \& Control }\end{array}$ & & \multicolumn{2}{|c|}{$4^{\text {th }}$ Graders } & $\begin{array}{c}6^{\text {th }} \\
\text { Graders }\end{array}$ & $\begin{array}{c}\text { No } \\
\text { Differences }\end{array}$ \\
\hline Tot Capacity Beliefs $\longrightarrow$ Coping & All & \multicolumn{2}{|c|}{$\mathrm{PS} / \mathrm{IS} / \mathrm{CF}$} & & ES \\
\hline Tot Strategy Beliefs $\longrightarrow$ Coping & All & & ES & $\mathrm{PS} / \mathrm{IF} / \mathrm{CF}$ & \\
\hline \multirow{2}{*}{ 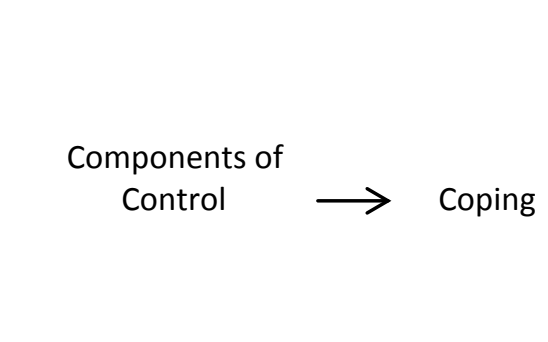 } & \multirow[b]{2}{*}{ PS / IS } & PS & $\begin{array}{c}\text { Ability } \\
\text { Pwrfl Oth } \\
\text { Luck }\end{array}$ & Effort & Unknown \\
\hline & & IS & $\begin{array}{c}\text { Effort } \\
\text { Pwrfl Oth } \\
\text { Luck } \\
\text { Unknown }\end{array}$ & Ability & \\
\hline Max Control $\longrightarrow \Delta$ Coping & All & PS / & $\begin{array}{l}\text { IS / CF / } \\
\text { ES }\end{array}$ & & \\
\hline
\end{tabular}

Analyses for this research question used the aggregate measures for perceived control for Total Strategy Beliefs and Total Capacity Beliefs. Correlations support the expectation that increases in strategy beliefs are associated with increases in helplessness coping, and decreases in mastery coping, while capacity beliefs are expected to show the opposite relationships. These patterns for the process links for the relationship between control and coping were obtained in all cases. 
With respect to the results of concurrent analyses, no age differences were found for Total Capacity Beliefs, but Problem-Solving coping in the fall showed a slight benefit from capacity beliefs for $6^{\text {th }}$ grade students in terms of outcomes, which was reversed in the spring with an increase in the strength of this relationship for $4^{\text {th }}$ grade students, resulting in an overall better outcome for them. Fourth grade students also had overall better outcomes for Information-Seeking and Confusion coping, as the influence of capacity beliefs on these ways of coping was found not only to be stronger than the $6^{\text {th }}$ grade students at concurrent time points, but also to increase in strength from fall to spring. Escape coping did not evidence any significant age differences at either time point, and did not change in terms of strength of influence for either grade.

A significant age difference was found for Total Strategy Beliefs predicting both ways of mastery coping, in the fall only. The overall strength of influence of this relationship was stronger for the $6^{\text {th }}$ grade students at both time points, but the increase in strength from fall to spring was greater for the $4^{\text {th }}$ grade students. By spring, no significant age difference was evident for either way of mastery coping. Overall outcomes for Escape coping were better for $4^{\text {th }}$ grade students at both time points, as the influence of strategy beliefs on this way of coping was stronger for $6^{\text {th }}$ grade students. No difference in outcomes was found for the relationship with Confusion coping in the fall, but increases in the strength of this relationship in the spring for the 
$4^{\text {th }}$ grade students yielded a better overall outcome in the spring for the $6^{\text {th }}$ grade students. These results are illustrated in Figure 33.

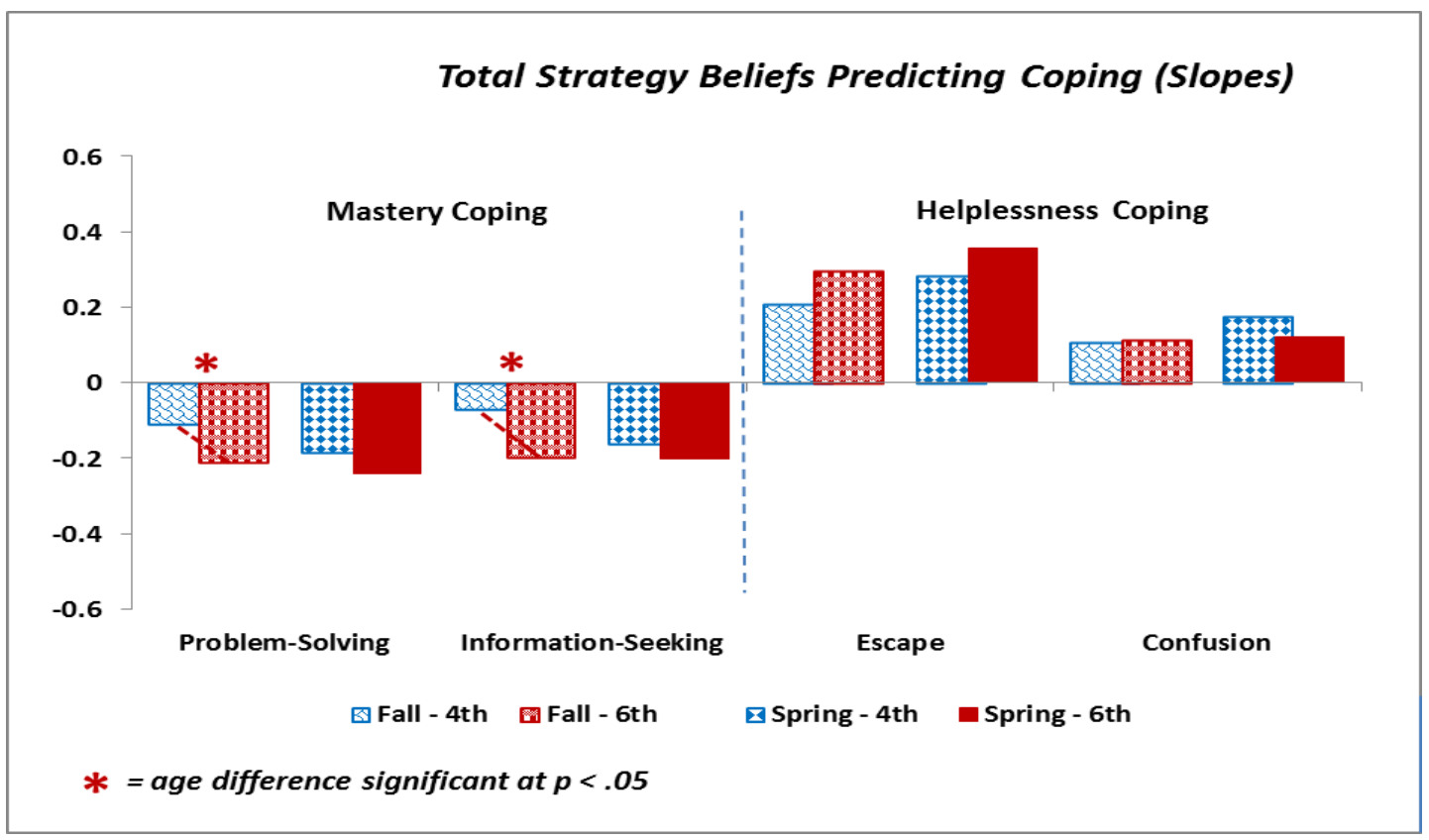

Figure 33: Total Strategy Beliefs predicting Coping; $6^{\text {th }}$ grade doing better than $4^{\text {th }}$ except on Escape.

Maximum control predicted changes in coping, with no significant age differences. Differences in outcomes showed stronger positive effects for all ways of coping for younger children. This is consistent with mean-level changes noted in control, in that $6^{\text {th }}$ grade students experience significant declines in the mean levels of their capacity beliefs, while $4^{\text {th }}$ grade students not only maintain strong capacity beliefs across time, but also make positive gains in the development of their strategy beliefs.

Further analyses with the disaggregated component measures for Total Strategy Beliefs to explore the finding of a significant age difference revealed 
evidence of the differentiation of Effort from Ability for the $6^{\text {th }}$ grade students, along with better outcomes for $4^{\text {th }}$ graders on Problem-Solving due to Ability beliefs, and Information-Seeking due to Effort beliefs. The results graphs showing the contribution of each of the component strategy beliefs to Problem-Solving and Information-Seeking are illustrated in Figures 34 and 35.

Two particularly interesting things about these findings stand out when results of the individual contributions of strategy beliefs to the development of mastery coping between the grade groups are compared. The first observation is that Strategy Beliefs for Effort has a positive influence on Problem-Solving coping for both grades, and on Information-Seeking for $4^{\text {th }}$ grade students, with a stronger influence on Problem-Solving as compared to Information-Seeking. The magnitude of the effect for Problem-Solving is stronger for $6^{\text {th }}$ grade students than for $4^{\text {th }}$ grade students, although not statistically significant. Compare this to the influence of Strategy Beliefs for Ability; the influence on Problem-Solving is positive for both grades, with a much stronger influence for $4^{\text {th }}$ graders, but the effect on Information-Seeking is negative for $4^{\text {th }}$ graders and positive for $6^{\text {th }}$ graders.

This observation is consistent with previous research that has defined the developmental trajectory of the differentiation of strategy beliefs (Skinner, 1990). By age ten, or about $4^{\text {th }}$ grade, children are able to distinguish between internal and external causes of success, and are moving towards differentiating within the internal 
Chapter 6: Discussion 312

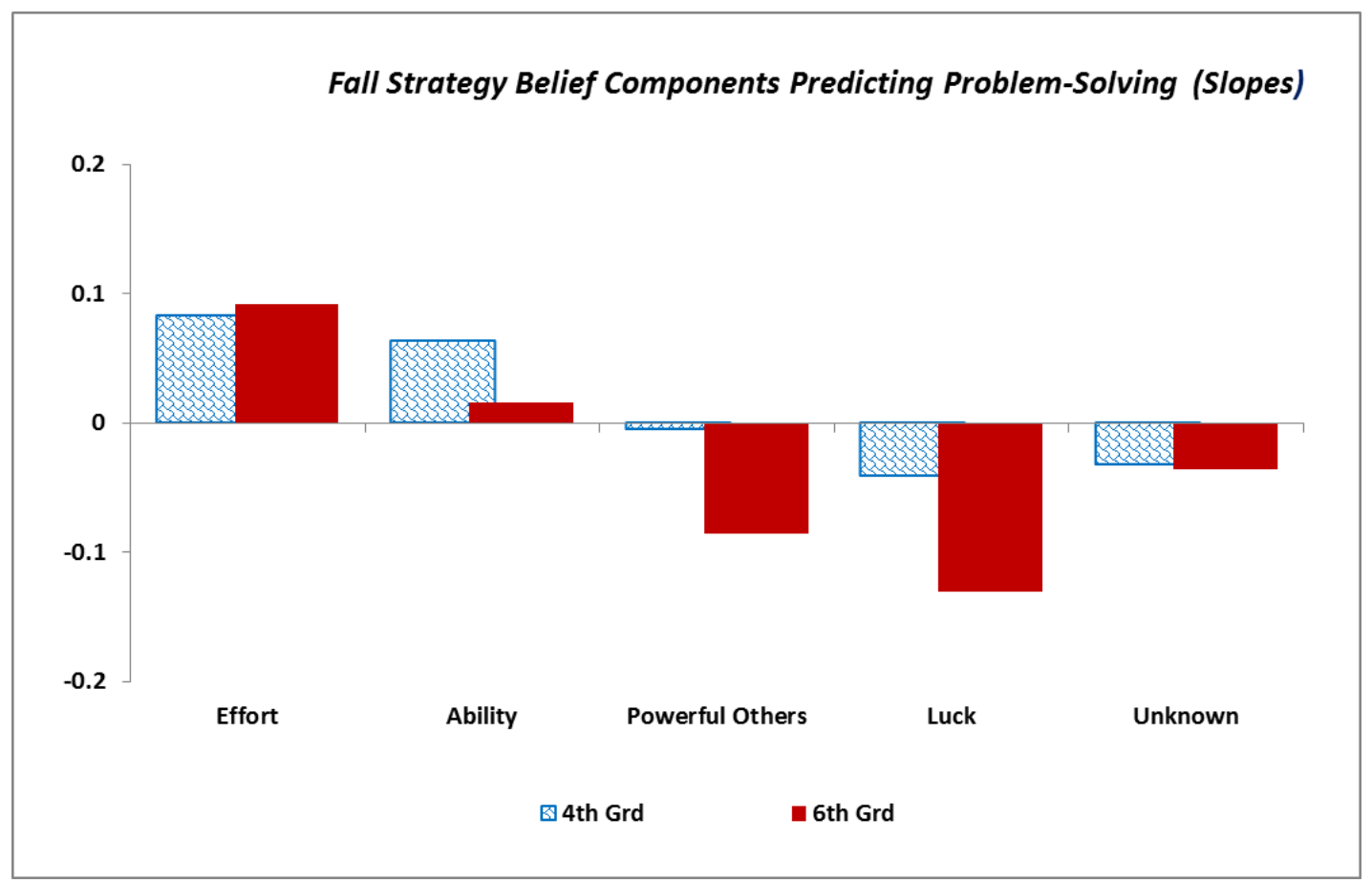

Figure 34: Strategy Belief components predicting Problem-Solving.

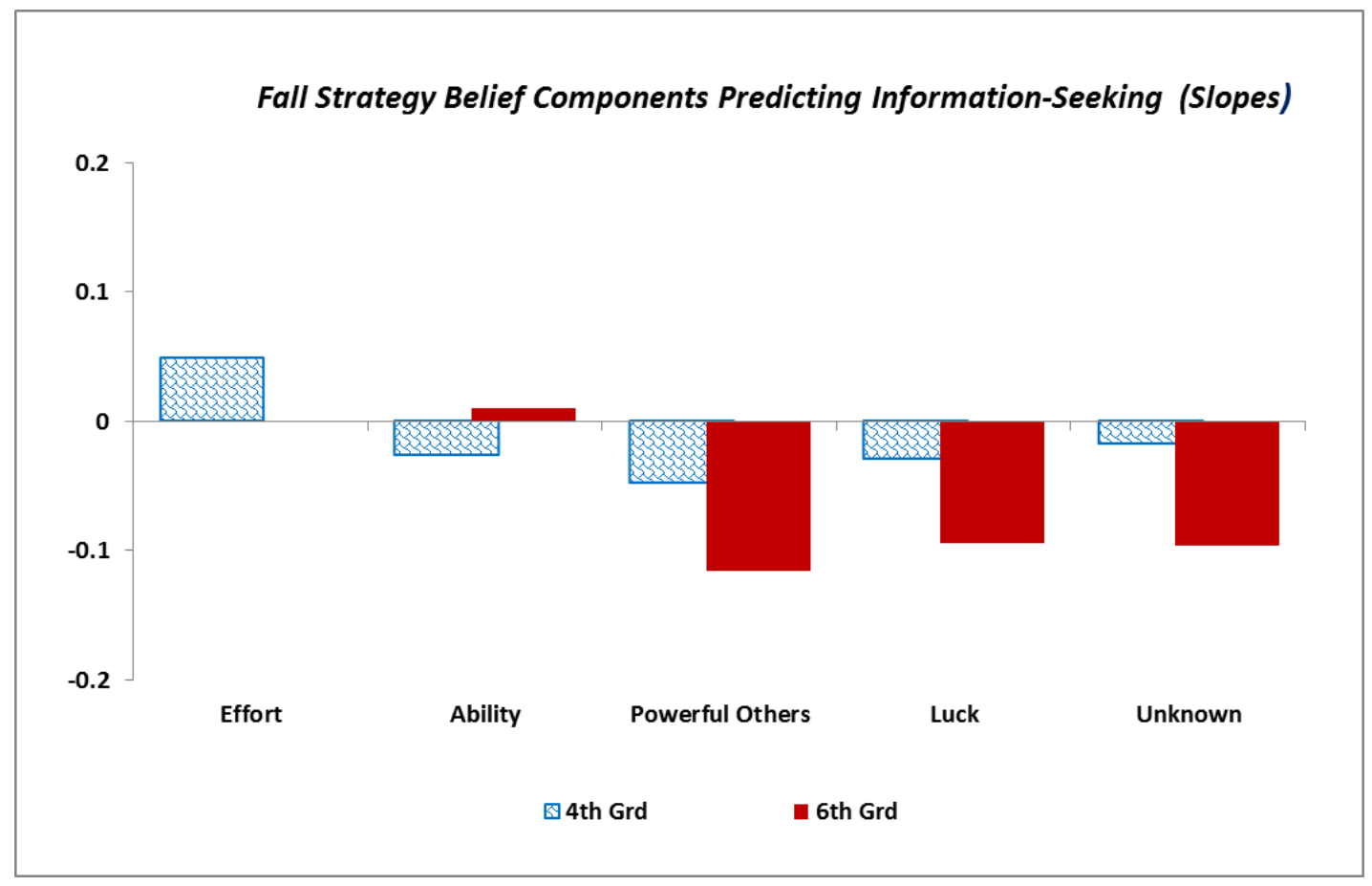

Figure 35: Strategy Belief components predicting Information-Seeking. 
category between personal effort and personal attributes (ability). These results illustrate this process and suggest that $4^{\text {th }}$ grade students are still considering effort and ability to be dependent causes, in that when they believe ability is the key to success, they are more likely to abandon information-seeking activities and move right to problem-solving. In contrast, $6^{\text {th }}$ grade students are farther along in the differentiation process and understand that effort is subject to internal control, but ability, even though it is an internal characteristic, is believed to be an enduring trait, and more effort does not always translate to more ability. A belief that ability is necessary for success will then cause these students to seek out more information about how to approach a task, or even how to compensate for any perceived lack of ability. A belief that effort is the key to success will lead older students more directly to problemsolving activity, as their belief in the efficacy of this strategy bolsters their confidence that instrumental action will accomplish their desired goal.

The second interesting observation when comparing these results is that while the effects for each grade group of the uncontrollable strategies are negative on both ways of coping, each component predicts differently by grade. Strategy Beliefs for Luck has a stronger influence on Problem-Solving versus Information-Seeking for $4^{\text {th }}$ graders, while just the opposite is true for the $6^{\text {th }}$ graders. Problem-Solving coping is influenced to approximately the same extent by Unknown strategy beliefs for both grades, but the influence for Information-Seeking is much greater for $6^{\text {th }}$ grade students as compared to $4^{\text {th }}$ grade students. Strategy Beliefs for Powerful Others is a 
strong influencer for both grades for both ways of coping, but is much more so for the $6^{\text {th }}$ graders as compared to the $4^{\text {th }}$ graders.

These differential patterns of influence for strategy beliefs reflect the differences between the grades in the strength of the patterns of correlations between these constructs, and correspond with observations made from the findings of this researcher's unpublished Master's thesis study (e.g., when children believe success lies with Powerful Others, problem-solving efforts tend to decrease). They are also consistent with the research defining the developmental trajectories for strategy beliefs (Skinner, 1990) with respect to the differentiation of importance of uncontrollable and unknowable causes, and support previous findings that as children get older, estimates of the amount of influence they have over their environment become more realistic.

Research Question 3: Teacher Support predicting Coping. Teachers play an important role in a child's life and can have a tremendous impact on their development through the provision of supports that help build motivational resources. This research question investigated the process link between teacher support and coping, and used the aggregate measure of Teacher Support in all analyses. Observations for the results of these analyses are summarized in Table 62 .

Teacher Support did not predict any way of coping except Escape coping, confirming the process link only for that model. This is most likely due to the low correlations between teacher support and coping found in this study. Outcomes 


\begin{tabular}{|c|c|c|c|c|}
\hline $\begin{array}{l}\text { Table } 62 \\
\text { Structural Relationships and Developmental Trends for Research Question } 3\end{array}$ \\
\hline Model Pathway & $\begin{array}{c}\text { Consistent } \\
\text { with which } \\
\text { coping models }\end{array}$ & \multicolumn{2}{|c|}{$\begin{array}{c}\text { Trends suggest things are getting } \\
\text { better for... }\end{array}$} \\
\hline $\begin{array}{c}\text { Research Question } 3 \\
\text { Teacher Support \& Coping }\end{array}$ & ES & $\begin{array}{c}4^{\text {th }} \\
\text { Graders }\end{array}$ & $6^{\text {th }}$ Graders & Differences \\
\hline Teacher Support $\longrightarrow$ Coping & IS & PS / ES / CF & \\
\hline Teacher Support $\longrightarrow$ Coping & NOT & IS & ES & PS /CF \\
\hline
\end{tabular}

favored the $6^{\text {th }}$ grade students, with more positive trends seen for all ways of coping except Information-Seeking.

There was also no significant prediction by Teacher Support of changes in any way of coping, although outcomes for this relationship were better for $4^{\text {th }}$ graders on Information-Seeking, and better for $6^{\text {th }}$ graders on Escape coping. There was no difference on outcomes between the grades for the impact of Teacher Support on changes in either Problem-Solving or Confusion coping. Figures 36 and 37 depict the results of these analyses.

The biggest fall-to-spring changes in the feedforward effects of Teacher Support are for Information-Seeking and Confusion coping for the $4^{\text {th }}$ graders, and for Problem-Solving and Escape coping for the $6^{\text {th }}$ graders. Note, however, that the change for the $6^{\text {th }}$ graders for Escape coping is a decrease in the impact of Teacher Support, not an increase as would be expected, meaning over time, the ability of a teacher to positively impact a child's use of Escape as a coping mechanism diminishes. This relationship is also illustrated by the positive relationship of Teacher 
Chapter 6: Discussion 316

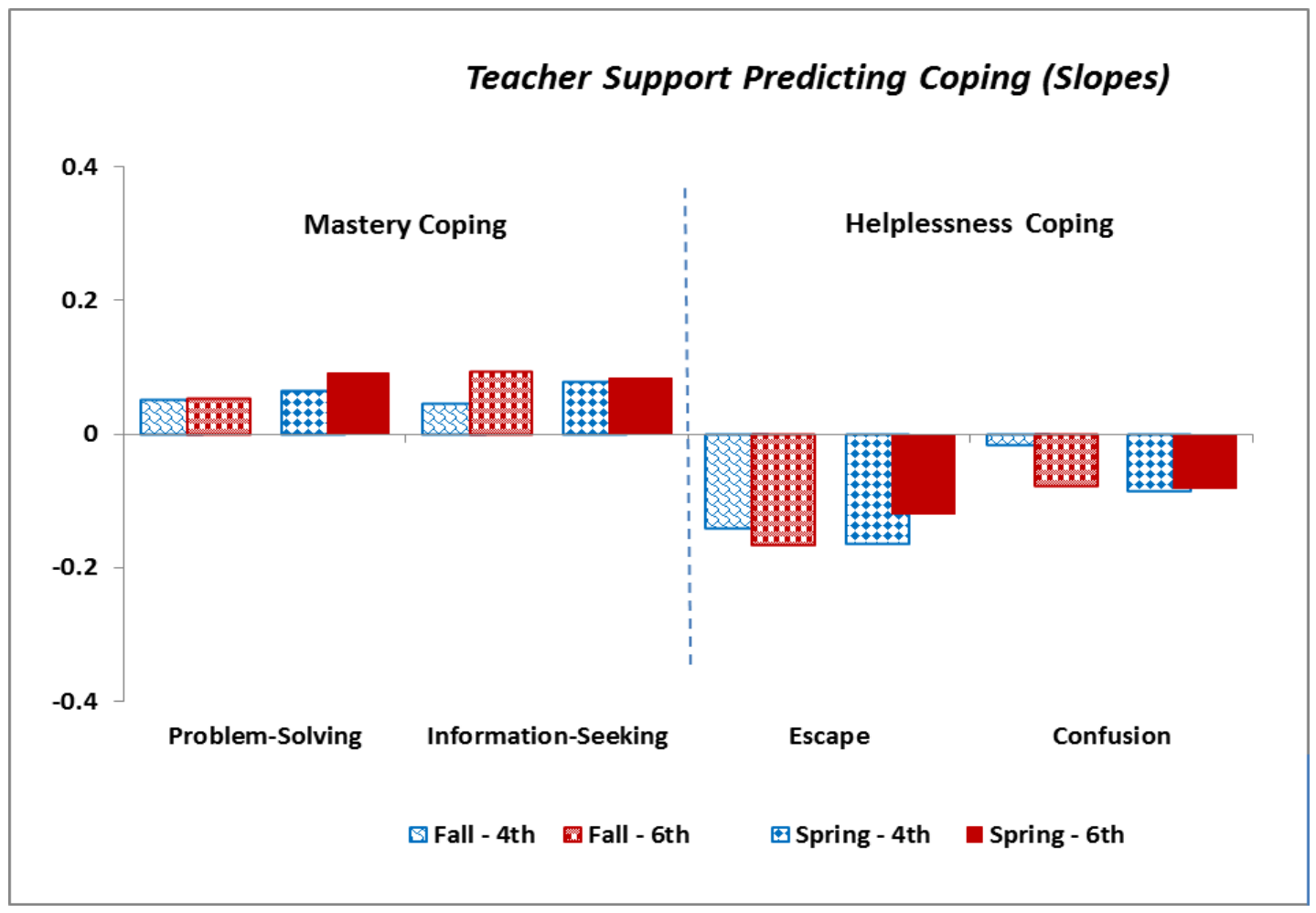

Figure 36: Teacher Support predicting ways of coping.

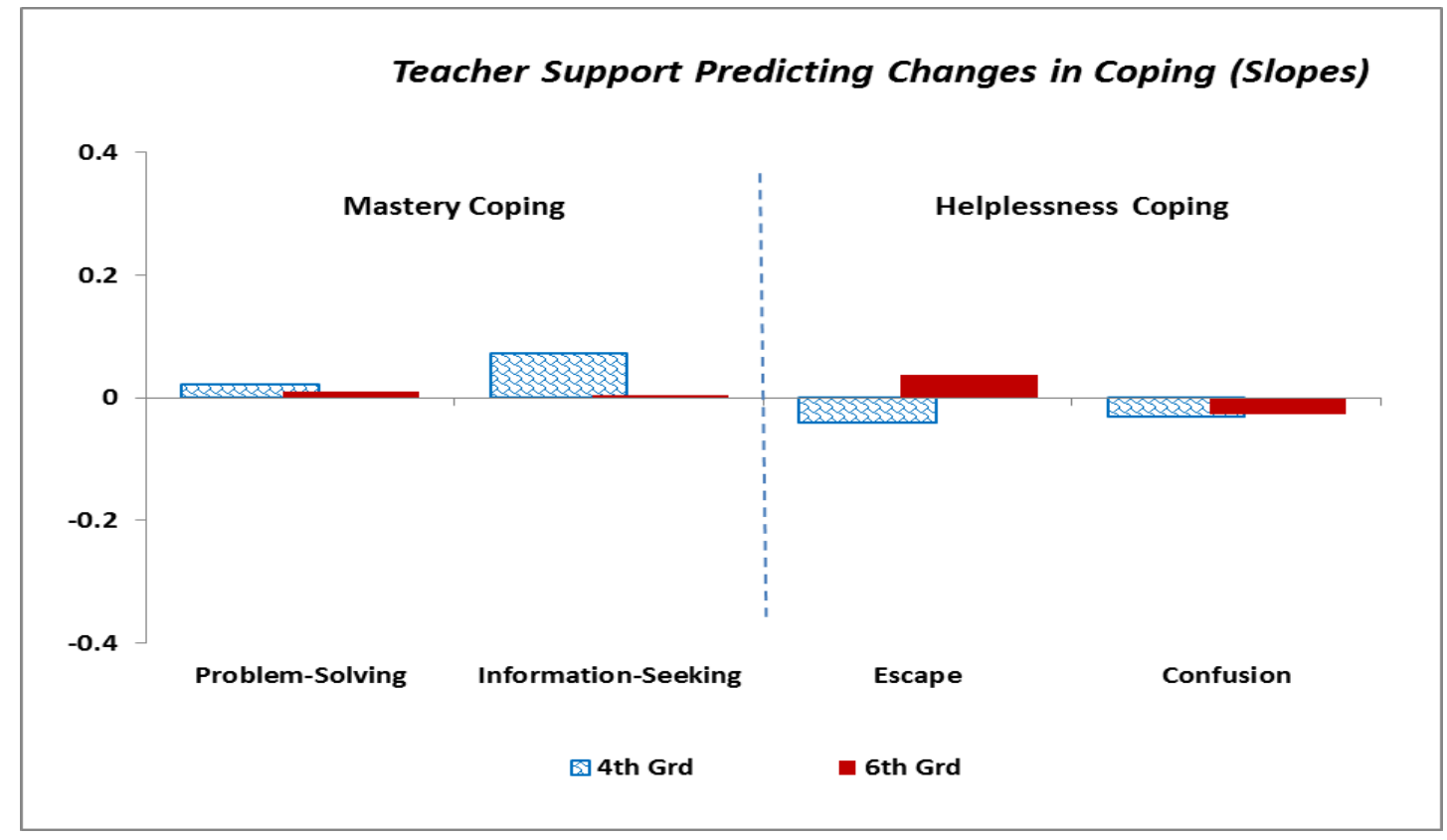

Figure 37: Teacher Support predicting changes in ways of coping. 
Support on changes in Escape coping. Note that the effect of Teacher Support on changes in coping is very small for all other ways of coping.

When considered together, the development of coping under the influence of Teacher Support follows these patterns: Teacher Support positively impacts ProblemSolving for both grades, exerting a stronger influence over time, and perhaps slows the decline of this way of coping noted in the mean levels. This effect appears to be slightly stronger for the $6^{\text {th }}$ graders compared to the $4^{\text {th }}$ graders; mean-level endorsement of Information-Seeking also decreases over time for both grades, and like Problem-Solving, may be slowed in that decline by increases in Teacher Support across the year, but only for $4^{\text {th }}$ grades, as $6^{\text {th }}$ grade students do not show the same increase in the strength of the relationship for this way of coping as do the $4^{\text {th }}$ grade students. Escape remains essentially flat over time for $4^{\text {th }}$ graders, and shows only a slight increase in the strength of response to Teacher Support. For $6^{\text {th }}$ grade students, Escape coping increases over time, and while Teacher Support does show an ameliorating effect on Escape, the strength of that effect becomes weaker over time; Confusion coping declines slightly over time for $4^{\text {th }}$ grade students, and the impact of Teacher Support in reducing Confusion increases across the year, but for $6^{\text {th }}$ grade students, Confusion coping continues to increase, while there is essentially no change across the year in the impact that Teacher Support has on this way of coping.

Overall, older students do not experience as much benefit from Teacher Support as younger students do with respect to the influence of support on coping 
behavior, even though the overall change in the mean level of Total Teacher Support was significantly greater from fall to spring for the $6^{\text {th }}$ grade students, and virtually flat for the $4^{\text {th }}$ grade students. In addition, the changes in baseline levels of coping, particularly Escape coping, appear to be changing for older children in greater magnitude than what the impact of Teacher Support is able to compensate for. This suggests that the support needs of $6^{\text {th }}$ grade students may be changing in ways that teachers are not recognizing as these children approach the transition to middle school and experience other physiological and social changes across the school year.

Research Question 4: Feedback effects of Engagement and Coping. Reciprocal effects models describe the dynamical nature of relationships that are iterative and produce either amplifying effects or dampening effects over time. This model has been used in the present study to conceptualize how motivational resources and coping repertoires may shape contextual features of the environment and personal resources over time. Engagement and coping were examined in this set of analyses to determine whether age differences exist in how the effects of these variables feed back into provision of support from teachers, and a child's own sense of control, to shape the development of these factors. The expectation is that these feedback relationships are reinforcing, producing an amplification of the effects with each trip through the cycle.

Coping behavior was found to have a significant feedback effect on changes in Total Strategy Beliefs only for the Escape coping model and only for Confusion coping for Total Capacity Beliefs. Coping did not predict provision of Teacher 
Support. Engagement was found to have a significant feedback effect on changes for all control beliefs, but not provision of Teacher Support. No significant age differences were found for any confirmed pathway, but overall, outcomes were better for $6^{\text {th }}$ graders regarding the feedback effects of mastery coping for both provision of Teacher Support and Total Capacity Beliefs, but better for $4^{\text {th }}$ graders on these outcomes for helplessness coping. This pattern differed for the effects on Total Strategy Beliefs; the $4^{\text {th }}$ grade students showed better outcomes than the $6^{\text {th }}$ grade students did for all ways of coping except Escape. No appreciable differences were found between the grades for the feedback relationships between Engagement and the control aggregates, but $6^{\text {th }}$ grade students did evidence better outcomes for the feedback effect of Engagement on changes in Teacher Support. These findings are summarized in Table 63.

All patterns noted for the direction of influence from coping to control or Teacher Support followed expectations, namely that increases in mastery coping result in increases in Teacher Support and Total Capacity Beliefs, and decreases in Total Strategy Beliefs and increases in helplessness coping result in the opposite relationships. Influence from coping to these constructs did not follow this pattern consistently by grade. Notable departures were for $4^{\text {th }}$ grade students regarding mastery coping predicting changes in Total Capacity Beliefs, which decreased slightly, yielding in fact an almost flat trend, and for $6^{\text {th }}$ graders on Problem-Solving predicting changes in Total Strategy Beliefs. When taken together with previous 
analyses that confirmed expected relationships in the opposite order of the constructs (e.g., control influencing coping), these results confirm the reciprocal nature of these relationships and support the notion of amplification cycles operating for each.

\begin{tabular}{|c|c|c|c|c|}
\hline \multicolumn{5}{|c|}{$\begin{array}{l}\text { Table } 63 \\
\text { Structural Relationships and Developmental Trends for Research Question } 4\end{array}$} \\
\hline Model Pathway & \multirow[t]{2}{*}{$\begin{array}{l}\text { Consistent } \\
\text { with which } \\
\text { coping models }\end{array}$} & \multicolumn{3}{|c|}{$\begin{array}{l}\text { Trends suggest things are getting better } \\
\text { for.... }\end{array}$} \\
\hline $\begin{array}{l}\text { Research Question } 4 \\
\text { Feedback Effects }\end{array}$ & & $\begin{array}{c}4^{\text {th }} \\
\text { Graders }\end{array}$ & $\begin{array}{c}6^{\text {th }} \\
\text { Graders }\end{array}$ & $\begin{array}{c}\text { No } \\
\text { Differences }\end{array}$ \\
\hline Coping $\longrightarrow \Delta$ Tot Strategy & ES & PS/ IS / CF & ES & \\
\hline Coping $\longrightarrow \Delta$ Tot Capacity & $\begin{array}{c}\text { NOT } \\
\text { CONFIRMED }\end{array}$ & ES / CF & PS / IS & \\
\hline Coping $\longrightarrow \Lambda$ Teacher Support & $\begin{array}{c}\text { NOT } \\
\text { CONFIRMED }\end{array}$ & ES / CF & PS / IS & \\
\hline Engmnt $\longrightarrow \Delta$ Tot Strategy & Confirmed & & & $\checkmark$ \\
\hline Engmnt $\longrightarrow \Delta$ Tot Capacity & Confirmed & & & $\checkmark$ \\
\hline Engmnt $\longrightarrow \Delta$ Teacher Support & $\begin{array}{c}\text { NOT } \\
\text { CONFIRMED }\end{array}$ & & $\checkmark$ & \\
\hline
\end{tabular}

Further examination of the results graphs illustrates one of these exceptions to the expected patterns of influence: Problem-Solving coping predicting changes in Total Strategy Beliefs for $6^{\text {th }}$ grade students yields a positive relationship, when an inverse relationship is expected. Figure 38 presents the graphical display of the results for coping predicting changes in Total Strategy Beliefs.

As can be seen, the pattern obtained for $4^{\text {th }}$ grade students follows expectations, but for $6^{\text {th }}$ grade students, as the values for Problem-Solving increase, 
Total Strategy Beliefs also increase. The feedback loop still appears to be a reinforcing loop, just in a different direction than expected, but the strength of the amplification effect over time is weak, due to the very small effect size. Nonetheless, this finding does give cause to suspect that the Problem-Solving relationship with control may operate differently by age.

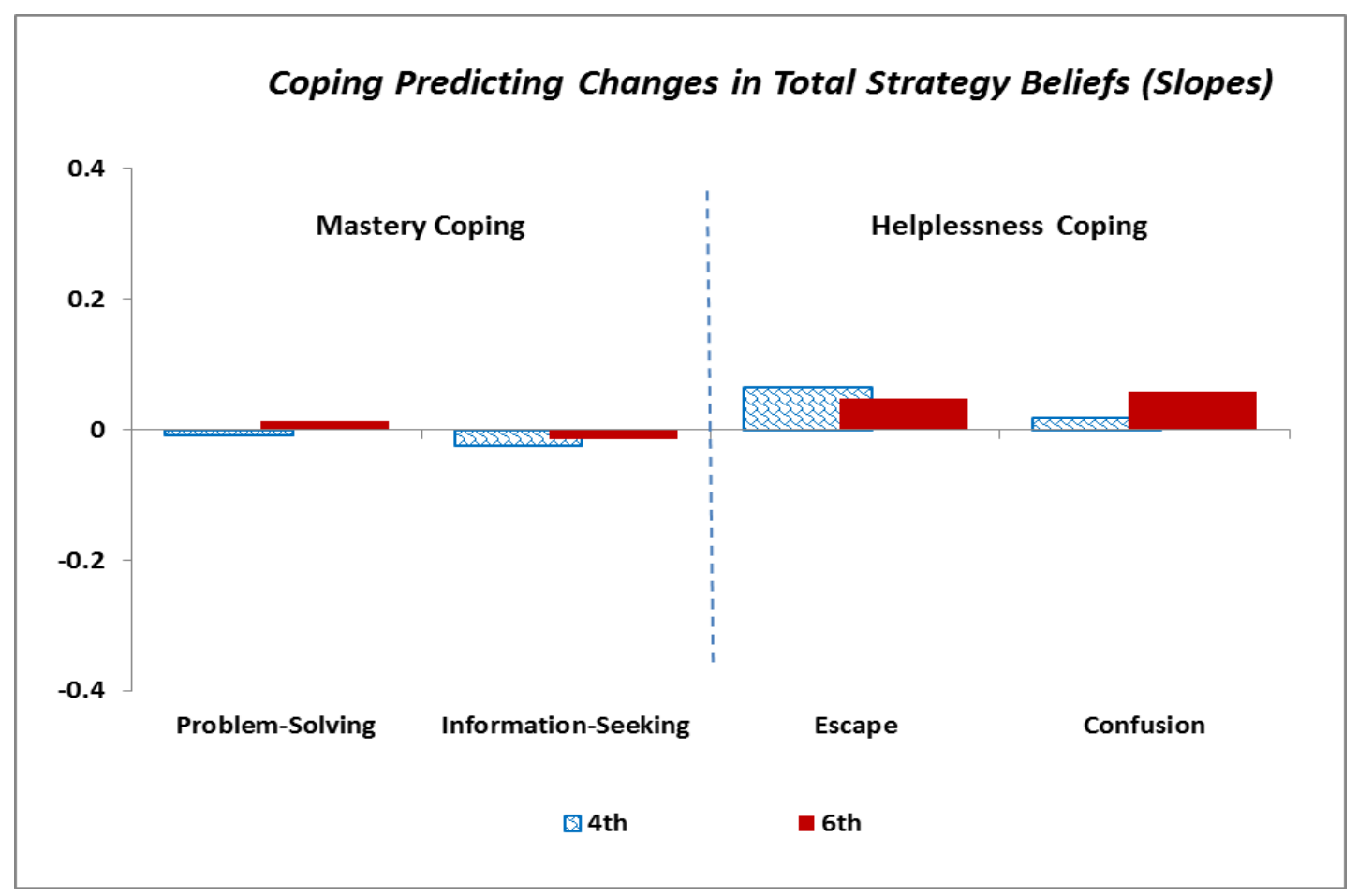

Figure 38: Coping predicting changes in Total Strategy Beliefs; PS positively predicts for $6^{\text {th }}$ graders.

Figure 34 (previously displayed, p. 312) showed the effect of the individual strategy beliefs on Problem-Solving. Recall that $6^{\text {th }}$ grade students showed a substantial increase in Problem-Solving due to increases in Strategy Beliefs for Effort, and that Strategy Beliefs for Effort was the only component that was significantly related to Problem-Solving (Table 41, p. 266). Also recall that the mean levels for 
uncontrollable strategy beliefs are increasing significantly for the $6^{\text {th }}$ grade students, while the fall-to-spring change in Effort and Ability are not significant (Table 10, pp. 228-229). The most likely explanation then for the finding of an inverse relationship in the feedback effect for Problem-Solving on Total Strategy Beliefs is that the change that is occurring in the control construct as Problem-Solving increases is in the Effort component only, and is of a sufficient magnitude to overcome the negative effects of the significant increase in the other components of the construct, thus producing an increasing trend in the overall construct rather than a decreasing one.

Summary and integration of findings. The present study examined a process model of the relationships between the internal resources of perceived control and coping, and academic engagement and achievement, and the role of contextual support from teachers in facilitating those relationships. Normative developmental trends for a sample of students in the $4^{\text {th }}$ and $6^{\text {th }}$ grades were noted and compared to describe how each of these constructs changes over time. A series of hypotheses regarding the structural relationships of the process model, and specific areas where age differences were thought to be significant, were also tested. Results indicate that in spite of the developmental shifts described by changes in the mean levels of the constructs, no significant differences by age were found, with only one exception.

In general, younger students are found to have high, stable control beliefs, moving towards more detailed differentiation of causes for success, confidence in their own efficacy to enact strategies that lead to success, with stable trajectories of mastery 
coping and high levels of engagement. When uncertain about how to proceed, younger children tend to cope by exhibiting confusion, rather than attempting to escape the situation. Their teachers are very supportive, providing increasing autonomy support across the year, while decreasing the amount of structure imposed.

Older children, in contrast, have less stable control beliefs, particularly with regard to uncontrollable strategies. While they have achieved a sharper differentiation between the strategies of Effort and Ability, they are less certain about the roles that uncontrollable strategies play, as beliefs for these strategies are increasing. At the same time, their confidence in their capacity to enact strategies for success is decreasing, and they tend to cope by escaping the situation when they are uncertain. Mastery coping in both forms is utilized, but less than as seen for younger children. Teachers are initially less supportive of older children, but tend to increase support in all areas, particularly provision of structure, as the year progresses.

While none of the hypotheses for this study regarding age differences were supported, many of the structural relationships of the model were confirmed. Closer examination of the trends detected in the process relationships provide clues to the dynamics of change that are occurring and provide valuable insight to developmental processes. The mean levels of each way of coping showed a pattern of endorsement where younger children were higher on their endorsement of mastery coping and lower on their endorsement of helplessness coping than the older children, but the pattern of changes noted in the process relationships illustrates that the direction of 
changes in coping are actually the opposite. A synthesis of these insights by way of coping, detailing the differences noted by grade, follows.

Problem-Solving. Problem-Solving appears to be more important to on-going Engagement for older children than younger children as increases in Problem-Solving for $6^{\text {th }}$ graders was greater than for $4^{\text {th }}$ graders. The more differentiated view of the $6^{\text {th }}$ grade students of Effort as a strategy for success is most likely responsible for this change, as Total Strategy Beliefs was the only construct in the model for which a significant age difference was found, with a stronger effect for $6^{\text {th }}$ grade students, and it was the Effort strategy belief component that was the only significant predictor of Problem-Solving when the Total Strategy Belief construct was disaggregated. The accompanying increases in Engagement did feed back into coping more strongly for older children as well, to reinforce increased use of this way of coping.

It should be noted that Total Strategy Beliefs as an aggregated construct has an overall negative effect on Problem-Solving for both grades, but more so for the $6^{\text {th }}$ grade students. It is only when the construct is disaggregated that it can be seen that it is the uncontrollable strategies that are causing that effect, as the strategies for Effort and Ability are positively related to Problem-Solving, and significantly so for Effort, with effects stronger for the older children.

Beliefs of $6^{\text {th }}$ grade students in their capacity to enact strategies for success translate into increases in Problem-Solving, which does feed back into strengthening Capacity Beliefs for older students more so than younger students, but may also be 
tempered by their growing uncertainty about uncontrollable strategies for success. Support from teachers, which is not particularly high at the beginning of the year for $6^{\text {th }}$ graders, does seem to have a slightly stronger impact on Problem-Solving for these students than it does for $4^{\text {th }}$ graders. The reciprocal effect of increased ProblemSolving does appear to have a stronger impact on teachers for $6^{\text {th }}$ graders, particularly with respect to provision of structure, which increases for older students across the year.

Information-Seeking. Even though Information-Seeking appears to be more closely linked to on-going Engagement for $6^{\text {th }}$ grade students, most other relationships with Information-Seeking in the model seem to favor the $4^{\text {th }}$ grade students. Both mastery ways of coping are more strongly related to changes in Engagement for $4^{\text {th }}$ grades students, suggesting that mastery coping is more influential for building Engagement rather than maintaining it for the younger students. As younger students develop more refinement in their ability to distinguish features of beliefs about causes of success, Information-Seeking appears to be a coping strategy they rely on more heavily than do older students, which feeds back into the positive aspects of their strategy beliefs. Overall high profiles of control in younger students lead to increases in Information-Seeking, more so for younger students than for older students, and support from their teachers appears to encourage this way of coping.

Escape. Escape coping did not evidence many differences in trends between the two grade groups. One important difference that did surface, however, was that 
Escape coping appears to be particularly damaging for $4^{\text {th }}$ graders with respect to changes in Engagement. The relative stability of strategy beliefs of $4^{\text {th }}$ graders does offer some protection against falling into this way of coping; however, this stability appears to erode more quickly for younger students who do use Escape coping than for older students, which sets up conditions for a reinforcing feedback loop between increasing negative strategy beliefs and increased use of Escape coping, which will ultimately have devastating effects on a child's Engagement.

A high level of support from teachers appears to guard against this pitfall somewhat, and might mitigate the potential of early adoption of Escape strategies by younger children. There is some evidence to suggest that for those younger students who do begin using Escape as a means of coping with challenges, the impact on provision of teacher support is more damaging than for older children, and may cause teachers to withdraw from students who exhibit this behavior.

Confusion. Use of Confusion coping by older children appears to be more damaging than for younger children in terms of impact on Engagement, but not as much as the use of Escape coping. The feedback effect that reinforces increased use of Confusion is much stronger than for Escape coping, but does not impact older students as much as it does younger students.

These tendencies may be reflective of the increased instability of uncontrollable strategy beliefs, and decreased capacity beliefs in older children. In fact, Confusion coping reinforces changes more for older children than for younger 
children in both belief sets in undesirable ways, with further increases in strategy beliefs and decreases in capacity beliefs. Younger students with a high control profile are less vulnerable to Confusion coping than older students with a similar control profile. Teachers appear to respond to Confusion coping equally between the grades, with little change in their provision of support.

Conclusion for summary of findings. These descriptions attempted to illustrate for which group the consequences of using a particular way of coping was more impactful, and how coping patterns are in flux over the course of a year. Mean level endorsement of the constructs seems to indicate that overall, younger students tend to use more mastery coping and less helplessness coping, while older children do just the opposite. As a consequence, Engagement is higher for younger children than older children, and control beliefs are more stable as well. Teachers are generally supportive, but more so for the younger children.

However, the dynamics of the process links tell a different story, or at least hint at the changes that are occurring that cannot be detected by looking at the mean levels. Older students are using more Problem-Solving, are experiencing more changes in their control beliefs as they reconsider their options for success and their capacities to access those options. These changing beliefs likely lead to increases in Confusion coping in addition to already high levels of Escape, but with bigger negative impacts, as Engagement continues to decline and teachers do not respond with the necessary levels of increased support. 
Younger students, on the other hand, are increasing their use of InformationSeeking, continue to believe in their capacity to enact strategies for success, even though their understanding of those strategies is changing. They tend to remain engaged unless they slip into patterns of Escape, which teachers do not respond to as well to as they do when students use Confusion coping.

Since there were no true age differences found in this study, though, these descriptions of the ways of coping and how they might operate most optimally for which grade is based solely on the pattern of trends indicated by very small effect sizes, as noted in the analyses. As such, they are subject to reinterpretation in the light of information provided by future studies and additional analyses. Further integration or attempts to overlay the findings of this study with the original four models obtained from this researcher's unpublished Master's thesis study are not possible due to methodological and design differences between the studies that impede comparison of the findings from each study.

There are a few strategies that could have been added to the present study to increase the potential for detecting significant age differences. Perhaps personcentered analyses that identify and compare groups of students whose characteristics on different combinations of the study constructs fit extreme profiles would have magnified the differences between the groups, making them detectable at statistically significant levels. 
One set of analyses that was included in the original study for this researcher's unpublished Master's thesis that was omitted from the present study was an investigation of the interaction of strategy and capacity beliefs. Applying the personcentered analysis model to this particular scenario would result in looking at differences between the grades for students who believed certain strategies would lead to success and believed they could enact those strategies, compared to students who did not know what strategies might lead to success, and did not feel they had access to any strategy to be successful. This analysis in particular may also have revealed particular points where significant impacts are occurring.

Relaxing the criteria for significance to include results at a p-value of less than .1 would have allowed for a few more results to be considered significant differences. There were only four other results that met this criterion; Total Strategy Beliefs predicting Escape coping (effect stronger for $6^{\text {th }}$ grade students), Strategy Beliefs for Ability predicting Problem-Solving (effect stronger for $4^{\text {th }}$ grade students), and maximum control predicting changes in Confusion coping (effect stronger for $4^{\text {th }}$ grades students) all were significant at $p<.07$. Feedback effects of Engagement on Confusion coping was significant at $\mathrm{p}<.08$, with effects stronger for $4^{\text {th }}$ graders. These nearly significant trends were included in the summarized descriptions of each way of coping provided earlier.

These are strategies that could have been implemented with the data available for the present study, as they were collected. Other strategies to address the lack of 
significant findings that involve things that could not be done, given the design features of the study, are offered in the next section in the discussion of the study strengths and limitations.

\section{Strengths and Limitations}

This study exhibits strengths and limitations in both theoretical and methodological areas. The discussion in this section highlights several of these in each area.

\section{Theoretical Strengths}

Theoretical bases. This study is founded on a set of established theories that tie together systems concepts into a process-oriented examination of motivational dynamics. The present study is particularly interested in those subsystems involved in the regulation of action, as defined by action theory, comprising behavior, emotion, and orientation (thought). Self-determination theory provides a conceptual framework to define the particular processes of interest within each of the three components of action - the self-system processes of relatedness, competence, and autonomy, the fulfillment of which provides both the energy and the impetus for goal pursuit. These three areas of regulation and three systems of related psychological needs interact with each other and with other chemically based regulatory systems in the body (e.g., the endocrine system, which includes the adrenal subsystem that controls stress-related hormone production) to coordinate the energy expenditure of mind and body towards 
desired goals. The quality of the interactions between these systems is a determining factor of the psychological state of the person as a whole at any given moment.

Focus on motivational dynamics. Underlying this framework is a systemsoriented perspective that hones in on the notion of motivational dynamics within regulatory systems, and emphasizes control processes, particularly feedforward and feedback loops and the amplification or dampening cycles they foster. Engagement, or motivation, is conceptualized as a dynamic process comprised of iterative behavioral episodes involving coping with challenges in the presence or absence of support from social partners in the academic context; the iterative nature of these episodes creates the system dynamics that ultimately drive the development of the constructs of interest, namely coping, control, and subsequent engagement.

Treatment of time. This study also draws on a conceptualization of time as a variable that represents both a progression of days, weeks, or months (chronological time), and a metric for normative shifts in development (developmental time). A distinction of this type is not commonly incorporated in studies that claim to be process-oriented. By looking at changes in the mean-levels of the constructs both within and between grades (average developmental change), while simultaneously examining the age-related changes in the patterns of influence between the constructs over time, both aspects of time are preserved. When both perspectives on time are considered, separately and in connection to each other, it becomes much easier to track the process of transformation of behaviors from one functional form to another. 
A systems-perspective. The present study draws on theories and concepts within the systems perspective in an attempt to untangle the complex interplay of the psychological aspects of regulation processes in order to understand how components of the system change and develop over time. The concepts of control systems, adaptive systems, feedback and feedforward effects, command functions, and machine programming all provide analogue models of components and processes within living systems. These are concepts drawn from the field of cybernetics, and understanding how the processes that operate in a cybernetic context compare and are different in humans can open the door to the formulation of new models of development. The systems perspective is particularly valuable in this regard in that it promotes a dynamic viewpoint on what sustains behavior, what limits it, and what causes it to increase with the passage of time on multiple scales, i.e., chronologically, and in the context of maturation. Understanding concepts like positive and negative feedback cycles and how they contribute to children's changing behavior patterns over time will ultimately guide concerned adults in knowing where and how to intervene to stop the "poor from getting poorer," and help children build new tendencies that create pathways to academic success.

\section{Methodological Strengths}

Study design. A longitudinal, cohort-sequential design was used for this study and is considered a strength, in spite of the difficulties usually associated with longitudinal investigations. The use of longitudinal data is a necessary feature of any 
examination of change over time, as there must be a baseline data point and at least one point collected later to use as a comparator. The cohort-sequential design strengthens any longitudinal investigation by allowing for the possibility of disentangling true age effects from cohort effects and other artifacts of the data collection process. A further strength of the design of the study is the use of multiple reporters. Both teachers and students provided data for this study, and the inclusion of different groups of participants helps mitigate the effects of self-report and commonmethod bias.

Treatment of missing data. Advances in methods and utility programs used to deal with the inevitable drawback in longitudinal studies of missing data have made it far easier than in years past to properly and accurately adjust study data for missing data patterns. Great care was taken in this study to first analyze the nature of the missing data patterns, and then select an appropriate means of treatment for it. Multiple imputation is the current gold standard in the field when hypothesis testing is planned, and there are many rules that govern the specification of the analysis set and the imputation set, which variables are included, which are excluded, which are combined, and myriad other considerations that must be made all along the process to arrive at a dataset ready for analysis. An extensive discussion is included in the Methods section of this dissertation that details the carefully thought-out decisions at each step of the process and the advice of missing data experts that support those decisions. The result was an analysis dataset that yielded minimal standard errors, and 
accurate estimates, which provides a high level of confidence in the accuracy of the results.

Treatment of mediation analysis. This study sought to test not only mediated relationships, but to also test for moderation of the mediated effect. In the literature this has been termed the conditional indirect effect, and a handful of experts in this area have debated over several years the best way to analyze complex models such as the one the current study proposed. The traditional model for testing mediation put forth by Baron \& Kenny (1986), has been dissected and looked at from a mathematical angle and found to be lacking. Leading researchers in the field of methodology today have advanced models and methods for analyzing these complex relationships that correct the shortcomings of Baron \& Kenny's recommendations (1986), including actual programs and macros for use with common statistical packages. This study made use of one such macro to test the conditional indirect effect of the relationships between coping, engagement, and achievement, to make a determination of whether age-graded differences exist in the mediated effect. No differences were found, contrary to expectations, although the general mediational effects were confirmed. Just as was done with the treatment of missing data, great care was taken in this study to understand and explain the details of this process, so as to provide an example of correct methods for analyzing these types of problems, and is considered a methodological point of strength. 
Normative development and individual differences. The present study addresses both intra-individual changes and inter-individual differences by combining an individual differences approach with an examination of normative development over time. A survey of the literature reveals a seeming lack of studies that utilize an analytic design that goes beyond the individual differences approach to describe both the influences that shape development as well as the process mechanisms of change as they operate together, in conjunction with normative change, leading some students to develop patterns different from the norm. Many studies that claim to be developmental or process-oriented are actually individual differences studies and do not incorporate an examination of normative development. By looking at the simultaneous progression of both these views on development, not only does it become more clear how contextual factors influence developmental processes, causing shifts in the normative trajectory of development, but information about what developmental processes are most active during what timeframes and in what sequences can also be noted. If shifts in the contextual factors cause delays in normative developmental process, or speed them up, the impact this deviation has on the pattern and sequencing of subsequent developmental episodes can be charted. This perspective is not often promoted in the literature, and the present study offers an example of the insights that can be gained from such an approach. 


\section{Theoretical Limitations}

Developmental constructs. While the collection of theories that provide the foundation for this study are impressive and substantial in their contributions to the underlying models being tested, the emerging theoretical formulation of motivational dynamics lacks some components that might be considered key. Goal orientations, both at the classroom level and the student level, have been included in discussions of academic motivation and achievement for decades (Ames \& Archer, 1988; Eccles \& Wigfield, 2002; Grant \& Dweck, 2003; Linnenbrink, 2005; Roeser, Midgley, \& Urdan, 1996). Further, goal orientations can be likened to the command function in a cybernetic control system. There is no direct human analog in the model posited in the current study, yet the command function is essential for the operation of a control system. Other motivational constructs that operate between perceived control and achievement may be at work that are not included in the theoretical planning for this study.

An additional theoretical shortcoming may be in the conceptualization of the construct of Engagement. While the construct as measured for the present study has been psychometrically validated (Furrer \& Skinner, 2003; Skinner et.al., 2009), and is adequate, it is a two-component construct comprising behavior and emotion, whereas other conceptualizations in the field suggest three or four components of engagement, and include the components of cognitive or academic engagement as well. The 
present study may have benefited from a conceptualization of engagement that included at least one additional component to tap the cognitive aspect of the construct. Methodological Limitations

Design issues. Even though the design of the present study is novel, and attempts a more micro-level analysis than is commonly seen in previous research, there are at least two notable limitations of the design itself. First, when considering developmental time, the span of months between the fall and spring measurement points represents a huge window of time during which children in this age group are experiencing significant normative changes. By collecting data at only two time points during the year, a good deal of information about the incremental progression of normative development is lost, making the results of within-subject analyses more global, and potentially less meaningful. It is possible that the processes of interest, especially ones involving feedback processes, are best captured on a smaller time scale, such as within weeks or days, or even hour to hour in the case of some types of interactions.

The second design limitation lies in having only one year of data to rely on for these analyses. The primary goal of this study was to examine age-related changes in the constructs of interest and the processes that relate them, and while a cohortsequential design was achieved, having only two data-points over the course of one year precludes a thorough investigation of inter-individual differences in conjunction with intra-individual changes. A design using a repeated measures approach with 
several measurement points across multiple years would allow for better comparison of intra-individual change over time between grades. Unfortunately, data are not available for multiple years for some of the constructs of interest, and this analysis is not possible.

Developmental window. The present study found no significant age differences in the process links relating the constructs of interest when looking for differences between $4^{\text {th }}$ and $6^{\text {th }}$ graders. It is possible that $4^{\text {th }}$ graders do not look different enough from $6^{\text {th }}$ graders to detect developmental differences. As someone once said, $4^{\text {th }}$ graders look a lot like $5^{\text {th }}$ graders, and $6^{\text {th }}$ graders look a lot like $5^{\text {th }}$ graders too. A wider developmental window may provide a large enough contrast between groups to allow for the identification of developmental differences. Future studies should consider comparing $3^{\text {rd }}$ to $7^{\text {th }}$ graders, or $4^{\text {th }}$ to $9^{\text {th }}$ graders. Moreover, with the shifting emphasis on statistical significance moving from the importance of a cut-off value for the p-value to deeper consideration of effect sizes and trends that act as markers for emerging significance, extended analyses in situations where significance is not obtained should be considered in order to understand the trends that are unfolding to produce change that will eventually be significantly different from past observations.

Data collection methods. In spite of the use of multiple reporters to address issues of bias, the data used in this study are self-report data, which is subject to a variety of biasing factors (e.g., social desirability, positive illusory). However, 
previous research has documented the reliability of the scales, and studies have been conducted to compare teacher and student reports and found sufficient congruence between the two reporters to confidently proceed. Had the self-report data gathered via survey instrument been augmented with other means of data collection, such as an observational protocol, the study may have gained a richer, more textured look at the constructs of interest, such as provision of support from the context. Observations of what teachers actually do in the classroom would provide information not only about the type of support provided by teachers, but also the nuances of how the support is provided, which could have an impact on how it is perceived by the student. Observations of student coping and student engagement might also provide a more nuanced view of these important processes.

Generalizability factors. The sample from which the data for the current study were collected was a predominantly Caucasian, upper middle-class sample, and this limits the ability to generalize the conclusions to other populations of greater ethnic diversity or differing SES levels. Having only one measurement point for the achievement measure, and then for only a subset of the total sample, further limits the generalizability of the results of the achievement analyses. The fact that the participants lived in a small town where there was only one school for their grades, and everyone in the town attended that school, further limits the generalizability of conclusions drawn based on the data collected from these participants. Many students do not attend school in a small-town setting. 
Age of the data. The data used for the present study were collected nearly twenty years ago. Changes in macro-influences (societal factors), such as communications technology, advances in personal computing, and access to the Internet, as well as changes in more proximal influences such as education policy and pedagogies, has likely shaped the generation of students currently moving through the school system in ways that were not present for the students from whom these data were obtained. However, the question here is not whether the students are different, but whether the changing world is able to influence the process structure that relates the constructs of interest in ways that would cause significant changes in the relationships between those constructs. An educated guestimate would be no, the process structure relationships are probably not influenced by the changing times such that engagement is no longer related to achievement, and coping no longer allows a student to deal effectively with challenges, reengage with schoolwork, or cause him to give up. The argument that the data are 20 years old, while compelling on the face of it, is simply not enough in and of itself to warrant new data collection efforts.

That said, what is more likely is that it is the teachers rather than the students who are different. Students most certainly are dealing with stressors of probably greater intensity in the new millennium than the later part of the $20^{\text {th }}$ century; but the patterns by which they respond to those stressors are likely to be the same. It is the teachers who have been subjected to new expectations (e.g., performance requirements for standardized testing), new evaluative models (e.g., changes in teacher evaluation 
practice and use), and new methods of teaching (e.g., Common Core Standards), who are now different in ways that may influence the outcome of these analyses. Not that changes in teachers would be enough to alter the process structure of the model either; however, if the goal is to understand how development proceeds and what factors influence it in the classroom context with teachers as social partners, then changes that have occurred for teachers would provide a solid rationale for conducting a new data collection so that a comparative study over the decades could be done to identify how the influence of teacher support has evolved. This effort would be most instructive if additional variables were collected from teachers on their own context, so an understanding of not only how teacher support has changed, but why it has changed, could be advanced as well.

If a new data collection were undertaken, it would be advantageous to include additional variables as previously discussed, expand the sample size to include multiple schools and districts, work to ensure a sample of greater diversity, and collect more of the study variables over more than one year, and more frequently within the year. Also, a careful consideration of the issue of developmental measurement equivalence, as described below, should be taken into consideration in any new data collection effort, and corrected with the addition of variables that measure the same constructs as they are expected to look at differing ages. This would allow for personcentered analyses and other comparative analyses to be conducted that would throw a brighter light on the change processes that are occurring. Further, it would provide the 
necessary comparison for commentary on the impact of changes in the educational landscape over the past 20 years.

Analytic methodologies. While the framing of this study from a systems perspective is a strength, the study falls short of capitalizing on all the systems perspective has to offer. A notable limitation in the methodology of this study is the linear treatment of the relationships of interest. The processes this study attempts to capture are largely considered to be dynamic and changing over time; however the analyses used to test the process mechanisms yield static information because they assume linearity in all relationships. The incorporation of an analysis of feedback relationships attempts to address this issue, but does not provide as much information as could be gained from non-linear consideration and analysis of the relationships in the model.

The field of systems dynamics offers a variety of non-linear trajectories to describe behavior and the interactions between variables. To identify these types of non-linear relationships, more sophisticated analytic techniques must be used, such as piece-wise growth curve modeling, or dynamical systems modeling using specialized modeling software. Valuable information may be lost by adhering to a strictly linear conception of the relationships of interest.

The selection of analytic strategies can also be critiqued. That is not to say that any of the analytic techniques used in this study were inappropriate. To detect the types of changes this study hoped to find, and then to identify developmental 
differences in those changes, may require additional analyses at a more micro-level. A look at how the factors that are believed to be causing the change are changing over time, and differentially by groups, may be necessary to understand how the change that is not being detected with typical hypothesis testing procedures is actually occurring. For example, if it is believed that differentiation between effort and ability is responsible for a change in the influence of control on coping, but control is not found to have the expected influence, then an investigation of the process of differentiation between effort and ability to see how it is progressing towards that point where it will cause the expected change may be warranted.

Another analytic technique that is gaining favor to address problems like those encountered in the present study in identifying change over time is person-centered analysis. With this technique, clusters of students who all display the same constellation of attributes are identified, and then those students are examined on markers of change. These techniques that differ from the traditional approaches are designed to magnify change processes that may be present, but that may have very small effect sizes that are difficult to detect at the point in time of observation.

Developmental measurement equivalence. According to the theories and body of literature detailing the findings of previous studies, certain patterns of changes were expected in the variables that did not materialize. For example, as children get older, Problem-Solving coping is expected to move from instrumental based problemsolving, to more cognitive forms. In addition, it was expected that there would be 
significant differences in the level of endorsement of Problem-Solving coping, with endorsement stronger for $6^{\text {th }}$ grade students due to advances in cognitive development as they approach the transition to middle school, resulting in greater use of cognitivebased Problem-Solving coping. This expectation was not confirmed, and it is possible that the way Problem-Solving was measured is to blame. All items comprising the measure of Problem-Solving are geared towards instrumental problem-solving and do not necessarily measure the cognitive bases for problem-solving. Thus, the expected finding was not detected - not so much because it wasn't there as perhaps because it wasn't measured. Future attempts to measure coping behaviors would benefit from preparatory work to formulate developmentally equivalent measures for a range of ages so that shifts in behaviors of the same function, but different form, at different ages can be more easily detected.

\section{Implications}

The combined wealth of information generated and translated from this study has both theoretical and practical implications. Theoretical considerations encompass the formulation of models of development and models of regulation, particularly through the lens of systems principles, and the impact this perspective may have on the study of regulatory processes. Coping as regulation, the study of regulatory processes in the academic domain, and teachers as not only social partners, but coregulators with students is discussed. Finally, practical considerations for the 
classroom gleaned from the insights of this study are offered, covering the continuum from K-12 through post-secondary study.

\section{Models of Development}

The literature is replete with studies investigating some aspect or another of development. A common and traditional tendency for programs of research is to consider different aspects of development as discrete sub-systems, modeled either as separate and independent encapsulated systems, or only at a single point in time. Longitudinal studies that integrate sub-units into larger interdependent systems that operate across developmental time-periods are not often undertaken, due to the financial and human resource costs involved in such studies.

Further, the investigation of differences in outcomes according to personal characteristics such as age, gender, or ethnicity is often limited to a description of mean-level differences and does not attempt to describe the shape of inter-individual trajectories of development over time. This could be due to the historical and strong reliance on established cutoff values for statistical significance that tends to stop an investigation short when those cutoffs are not met, thus precluding further investigation of differences over time. This emphasis has been changing in recent years to place more credence in effect sizes and trends rather than absolute criterion values, and this study provides a good illustration of the utility of adopting this new emphasis. Few statistically significant age differences were found in the present 
study, yet ample evidence for the strength and direction of developmental trends that provides insight into the process leading to emergent age differences was noted.

The present study also offers the advantage of advancing a line of questioning based on an integrated model of developmental constructs. Perceived Control and Engagement are two constructs that have been widely studied and have become firmly established in the literature as to their composition and expected influences. Perceived Control is understood to comprise beliefs about effort, ability, external causes of success, and the extent of controllability and agency in a variety of contexts, with the pathways to positive or negative outcomes well predicted and understood (Compas, 1991; Musher-Eizenman, Nesselroade, \& Schmitz, 2002; Skinner, 1995; Skinner et al., 1990; Skinner et al., 1998). Engagement has been documented extensively as a protective resource in the academic domain that leads a student to the experience of achievement (Christenson, Reschly \& Wiley, 2012). In spite of this wealth of research, the usual direction of influence that is tested, either on or from Engagement or control, is a feedforward effect, moving in only one direction. Reciprocal effects are rarely considered, and even more infrequent are discussions of multiplicative effects between these subsystems and other subsystems.

The present study was purposeful in calling upon a theoretical model that accounts for reciprocal effects and does not assume a unidirectional pathway of influence. Coping, one of the primary constructs of the study is conceptualized as an episodic process, implying that an interaction with the environment will occur and 
produce a measurable effect on other parts of the regulatory system that have consequences for the next interaction with the environment. Accounting for the ways in which each encounter shapes the resources available to effectively meet the challenges of the next encounter is essential to mapping the dynamical evolution of the component parts of the system and their subsequent effect on other parts of the system. The analyses conducted in the present study sought to chart these outcomes and subsequent influences, and indeed was able to track the progression of influence from one episode to the next. Confirmation of the operation of both feedforward and feedback effects was obtained, providing insight to the direction of developmental trends as children move through childhood towards the transition to middle school.

Incorporating a systems perspective. Reciprocal effects models allow for the investigation of change over time that includes the reinforcing or dampening effects of iterative feedback. The concept of feedback cycles is inherent to a systems perspective, and can offer deep insights into the progression of development, normative or otherwise. Humans conceptualized as dynamic systems, comprised of smaller sub-systems that are tied together by fluid, dynamical interactions is at the heart of a systems perspective on development. Systems, in general, are comprised of sub-components; the human organism is comprised of inextricably intertwined regulatory systems that operate on multiple levels and function according to a symphony of feedback loops. This impossibly complex network of feedback signals works together to maintain a homeostatic balance, within often narrow margins of 
viability, which allows for the continuance of life, and when optimized, promotes productive growth at both the physiological and psychological levels. The concept of feedback loops alone, however, is insufficient by itself to describe the complexity of human development.

When considered against this landscape, development becomes a process that suddenly must certainly exhibit other characteristics of complex systems - nonlinearity, sensitivity to initial conditions, boundaries that separate system from environment and define the person-environment context, and of particular importance to the present study, autopoieses (self-construction). Models of reciprocity, feedback loops, and the normal linear methodologies used to analyze them suddenly become woefully inadequate to address the necessity of investigating the simultaneous action of multiple components when exposed to patterns of multiple influences that in fact affect the interactions of interest themselves in a variety of ways. The methodological implications, then, of a truly systems-oriented, dynamical view of development requires the formulation of ever-more complex mathematics and simulation techniques in order to make sense of the ordered chaos one finds, along with the computer technology to make those methodologies accessible and useable.

Finally, the process of autopoiesis, or self-construction, in which function becomes subordinate to the self-organizing needs of the organism, describes the notion that humans as living systems are the producers of their own development (Lerner \& Busch-Rossnagel, 1981). To be self-constructing means an organism is equipped with 
the processes and components necessary to continuously and recursively produce the same types of components necessary to feed the involved processes. The present study highlighted a confusing set of circumstances wherein no age differences were detected in the process structure of relationships among the constructs, and yet, clearly there were significant differences in the mean-levels of the constructs over time, and notable developmental trends in the construct relationships that differed by grade. This begs the question then, if there are no age differences, where do these mean-level differences come from?

The systems perspective offers the possibility that they are the result of an autopoietic process, and the children themselves are the producers of their own development. That is not to say that this process occurs in a social vacuum. Human beings are necessarily embedded in multiple social contexts from which they draw the energy and materials to fuel the constructive process. Social interactions provide the raw materials that feed back into the system to activate the flexible nature of the operating rules so the person can adapt to changing environmental conditions. The interactions of the person with their environment, in conjunction with the influence of a person's particular genetic predispositions, are what produce development; thereby people are the producers of their own development.

Think of regulatory systems as the components and the processes they coordinate as the output, or development. The external and internal inputs of motivational resources and cognitions feed the regulatory process and produce 
changes in the regulatory structures and consequent behavior (development). If the inputs are of the right type and quality, the regulatory system is bolstered and strengthened; if the inputs are not there or are of an inferior or unknown quality, the regulatory system is weakened, a reorganization of the regulatory processes is required to adapt, and failures in regulation may result. It is this ability to self-organize and self-construct that distinguishes living systems from mechanical ones, and highlights the differences in the mechanistic versus contextual metatheories that guide individual researchers in their investigative process.

Summary of models of development. Models of development have followed a pattern of investigation whereby constituent subsystems have traditionally not been tested as an interactive component in a complex dynamical system. Additionally, individual differences studies have been modeled from the viewpoint of mean level differences, not an integrated perspective that could account for the interaction of multiple influences in integrated subsystems operating in an episodic manner across developmental time periods. The current study took a small first step in laying out an example of how this might be accomplished by bringing together related developmental constructs representing interactive systems and searching for the dynamics of the larger system as a whole. A systems perspective is instrumental in achieving this, and the incorporation of an examination of reciprocal effects and the identification of iterative feedback cycles illustrates the utility the systems perspective brings to the study of development. In the current study, the mere fact that most 
reciprocal relationships were found to be amplification cycles implies that indeed development is happening. Development implies change, and a key type of change is the amplification of an effect. Negative feedback loops describe dampening effects that serve to maintain equilibrium or bring a system back to a state of homeostasis, which can also be thought of as a type of change, a return to or motion towards a previous state, rather than a change of a developmental or transformational type. In the absence of the identification of negative feedback effects in the present study, one must assume that in spite of the lack of statistical age differences, development is in fact occurring.

\section{Models of coping and regulation}

The emphasis placed on the identification of reciprocal processes and the conceptualization of coping as "regulation under stress" used in the present study (Skinner \& Wellborn, 1994) has implications for wider exploration of regulatory processes from the perspective of developmental systems theory. From this vantage point, regulatory systems are conceptualized as networks of feedforward and feedback cycles. The contribution of the action-theoretic perspective defines the purpose of regulation as providing the coordination of behavior, emotion and orientation. Consider the case of Escape coping in this framework. It is the only way of coping found in the present study to be directly associated (negatively) with Achievement. Escape as regulation of action under stress might look something like this: behavior not participating; emotion - high distress; orientation - catastrophic thinking. The 
primary function of Escape as regulation then appears to be as regulator of emotion and attendant thought, in order to allow the person to return to a state of engagement. The next question this raises then from a regulatory perspective is one of identifying the process by which the transition back to a positive state of engagement is made.

The present study looked at feedback relationships between pairs of variables. What was found were reinforcing relationships - each variable is seen to be endlessly moving in the same direction in response to a unidirectional change in the other. However, nothing can increase or decrease infinitely; everything has a limit. In these particular loops, a third variable would have to be introduced in order to activate the limiting function. In the case of Escape as regulation, where increases in Escape promote increases in disaffection, what is the intervening variable that limits the increase in disaffection, and how does the student return from their Escape episode?

In the model used in the present study, that intervening variable is the teacher. As a socially embedded process, coping occurs in the context of social interactions (Aldwin, Skinner, Zimmer-Gembeck, \& Taylor, 2011). The limiting influence then to an episode of Escape and disengagement is some action from the teacher that brings the student's Escape behavior to an end and encourages them to pick up again with task participation, helps them soothe their emotions, and counters their worst-casescenario line of thinking, preferably with input that builds their capacity beliefs and focuses them on controllable strategy beliefs. The present study did not test the impact of teacher support on control. This would be an interesting avenue to explore 
from a perspective of regulation, and in response to the question of how a student is able to reengage after an episode of extreme coping such as Escape. Other factors that serve as intervening variables that introduce a limiting function to amplification cycles need to be identified to give a more complete picture of coping as regulation.

There are two additional questions of importance to consider: 1) how is a student able to bring themselves out of an extreme coping episode before the development of formal operations; and 2) can a regulation episode be deconstructed into a sequence of multiple coping episodes?

With respect to formal operations, the ability to think in terms of cause and effect, to anticipate outcomes, make alternate plans of action, and conceptualize multiple aspects of a problem or challenge are all tools that can be used to great effect in conscious regulatory processes, and are particularly important to efforts to bring oneself back to a positive place after a negative experience. Pre-adolescent children do not typically exhibit these advanced cognitive abilities, and are at a disadvantage when it comes to efforts to self-regulate in conditions of extreme distress. What the present study is unable to show is how a child who habitually uses Escape coping and is highly disaffected in the classroom can return to task participation and reengagement with the classroom context, with or without external assistance. Measures of cognitive development would be most interesting to correlate with ways of coping, especially across the transition to high school, or from early high school to late high school, when formal operations is typically emerging. This might provide 
insight to teachers on how to assist their students in developing the skills they need to boost motivation for academics through building self-regulatory capacity.

The second question regarding sequences of coping episodes highlights another important implication for how the question of what facilitates transition out of extreme coping episodes is approached. In considering the problem, one must assume a sequence of events that involves multiple coping responses. In simple language, if a student is disaffected in the present, the implication is that there was a time in the recent past when they were not disaffected, and the goal for the future is to create the conditions that allow them to return to that state. The present study considered conditions on a variety of variables on two different occasions, separated in time by several months. This methodology does not allow for the type of analysis required to identify and track transitions from one coping episode to another. It is this type of investigation, however, that would provide rich insight into regulatory episodes that very likely encompass multiple coping episodes.

A further implication for coping and regulation that can be drawn from the present study involves the surprising and somewhat troubling findings that teacher support was not strongly related to coping. It is possible that teacher support, measured as provision of structure, autonomy support, and involvement reflects better the supports needed for the development of the control beliefs, as the control beliefs are the proxy measure for the competence system. Again, teacher impact on control was not investigated in this study. As a socially embedded regulation process, coping 
should be impacted by interactions with the teacher, but the results of this study imply that there may be other contributions from teachers, from the perspective of regulation, that are more influential in the development of coping as action regulation under stress.

Teachers conceptualized as co-regulators with students may provide new insight to the relationship between students' regulatory processes and their context. Teachers are individuals acting in their own school context, with their own set of control beliefs, who, like students, also experience challenges and setbacks. A particularly salient challenge to a teacher's motivational system is dealing with students who may be poor regulators themselves and are chronically disaffected. Inability to positively impact these students can be very detrimental to a teacher's sense of efficacy and can result in what is reported as "burn-out."

Coping repertoires of teachers may serve as models, intended or otherwise, to students for their own coping behavior. Support may be offered in the form of soothing assistance when emotions are distressed. The measures of teacher support, as structure, involvement, and autonomy support, if reflective of behaviors that are consistent with co-regulatory behavior, coupled with an analysis plan that seeks to understand the interaction of the different feedback processes operating on both sides of the interaction instead of just one side, may advance understanding of regulation within the parameters of the underlying model used for the present study. 
Summary of models of coping and regulation. Coping as regulation under stress, in light of the findings of this study, or lack thereof, give rise to a set of questions that point to new perspectives on the underlying model of the present study and what it can contribute to the study of regulation. Before developmental differences can be tracked, several additional considerations can be made from the perspective of regulation. First, a fuller understanding of what regulation processes look like and how they operate in the academic context so they can be operationalized and measured should be incorporated into the underlying model. Next, a plan for exploring what the role of coping as regulation is and how it unfolds to define a regulation episode, including measuring and analyzing multiple coping episodes should be outlined. Then, how teachers, as partners in social interaction and coregulators with students, impact control processes, develop in their own right, and provide regulation assistance to students in transitioning between coping episodes should be added to the processes already measured by the current teacher support measures. These added features to a reconceptualization of the current study would provide a rich and nuanced view of development of a variety of internal resources.

\section{Educational Contexts}

Teachers as social partners provide information to students' regulatory feedback processes in a somewhat different way than do family members or peers. Parents or caregivers are a child's first social partners, providing protection from or buffering against the effects of stressors, modeling of adaptive coping strategies, and 
instrumental coping aid to build a sense of self-efficacy in subsequent stress encounters (Aldwin, et al., 2011). Peers and siblings provide additional examples of both what to do and what not to do to be effective in interactions with their environment and how to build social competence, as well as opportunities for a reflection of their developing sense of self. The gap left for teachers to fill, in addition to acting as surrogate to provide some or all of what a child's other social partners do, is that of mentor or guide in students' experience of their emerging self-reliance, sense of responsibility for one's own outcomes, and identity as an individual.

Elementary school teachers are tasked with creating an environment that encourages children to develop an enjoyment of the learning process, which is reinforced across the year so it becomes an enduring attribute. Teachers in the middle grades must find a way to encourage in students an acceptance of responsibility for their own learning without creating the experience of abandonment or helplessness. High school teachers face the challenge of developing confidence and autonomous decision-making for the future in their students, while providing a safety net in case they falter that is invisible until they need it, and does not promote the damaging effects of failure feedback. Post-secondary teachers are increasingly faced with the same types of challenges with adult learners of all ages, particularly at open-access institutions, where so many students arrive ill-prepared to meet the challenges of college level study and fall victim to a crushing sense of inadequacy and failure. 
These are difficult tasks laid at the feet of the nation's educators. The present study and others like it that deal with the notion of motivational resources, perceptions of control, and strategies for coping with academic challenges provide insights to the psychology of success, and identify the points of leverage that can build a student's motivation for learning and lead them to increased levels of achievement. The lack of association in the present study between teacher support and ways of coping and the absence of feedback effects on teacher support from any construct suggests that it is not well-understood how teachers can best partner with students to increase motivation and academic success. It should be noted, however, as was stated earlier, the data used for this study were collected nearly 20 years ago, and while age of the data may not impact the way the process links of the model function, it does raise the question about what teachers today know now that they may not have known then.

Teacher preparation programs typically include some instruction regarding the psychological processes whereby effort and ability attributions become differentiated, and the topic of differentiated instruction according to ability levels within the same classroom is addressed. But for new teachers, who may or may not have witnessed this developmental process first-hand via their own children, or through some other experiential learning opportunity, this concept is difficult to grasp, and is probably not recognized while it is occurring, nor overtly addressed by intentional action on the part of the teacher. This is not the fault of the teacher, and it is not the fault of the teacher's preparation program. It is simply indicative of the fact that until recently, 
there has not been a wide circulation of evidence, such as what is translated by studies conducted in the academic domain similar to the present study, to support professional development activities for teachers that give them the skills needed to proactively and positively influence these developmental processes in students.

Post-secondary teachers also can benefit from an exposure to psychological factors that influence success. Some research has been done showing the positive impact of perceived control on academic outcomes in the college setting (Doron, Yannick, Boiché, \& Le Scanff, 2009; Fishman, 2014; Perry \& Magnusson, 1989; Perry, Hladkyj, Pekrun, Clifton, \& Chipperfield, 2005). Recent reconceptualization of attribution retraining models as "mind-set" or "growth" interventions that aim to change students' thinking about the causes of success to more adaptive patterns have proven successful with high school students and have begun to surface as interventions in community college settings (Paunesku, et al., 2015; Yeager \& Dweck, 2012). Formalization of these types of cognitive interventions would be a simple, yet effective, method of addressing student performance gaps in community college settings where money, time, and expertise for implementing student success interventions is in short supply. Training for post-secondary teachers in the use of cognitive strategies such as fostering a growth mindset, encouraging adaptive coping, and providing targeted personal support should be provided, including the theoretical background that underlies the present study regarding what impacts positively on the motivational system. Not only would this kind of training benefit them as teachers 
practicing a craft, the success of which is measured largely by student performance, but they would accrue benefits personally as they were given the opportunity to reflect on how these processes impact their own sense of control and competence as they work in partnership with their institution, their students, and other individuals to transform the lives of adult learners.

Summary of implications. The present study provided an example of how the investigation of developmental processes can be advanced through application of a systems perspective. The present study offered an integration of developmental constructs based on a theoretically grounded model of interaction that is able to describe intra-individual changes and inter-individual differences, as well as account for reciprocal relationships among variables. Feedforward and feedback relationships are the foundation of the reciprocal relationships tested in this study, and allow for the consideration of regulation as analogous to cybernetic control systems. In addition, the systems perspective highlights how humans as living systems are dynamical, nonlinear, self-constructing, and self-organizing. These concepts, along with the notion that humans are the authors of their own development, open the doors to a rich field of inquiry that holds promise for untangling complex dynamical interactions that drive development, such as the myriad processes of regulation.

Coping, as a socially embedded process, is conceptualized as action regulation under stress, and as such is seen as a regulatory process in its own right. This implies that in addition to the feedforward and feedback cycles identified by the present study, 
there are additional intervening variables that come into play that serve to limit amplification of effects, and make true regulation possible. This leads to the idea that coping episodes, when analyzed as sequences of regulation, are the building blocks of various regulation processes. Several questions arise from these ideas regarding the capacities of children for self-regulation, the role of teachers as co-regulators, and the impact of teachers' own coping and control profiles on their interactions with students.

Practical applications in the classroom include wider recognition of how training and understanding of the principles and outcomes highlighted in this study can assist teachers in the often difficult tasks they are presented with to balance personal needs of students with performance expectations, as well as their own needs for experience of mastery of control. Teacher preparation programs, while they do cover some related concepts, could be more intentional about including detailed coursework on the psychological theories that describe developmental issues for children from a motivational resource perspective. Post-secondary institutions, particularly open-access institutions such as community colleges could also benefit from interventions to promote student success based on the principles and theories of perceived control and coping as motivational resources, especially those aimed at retraining students' conceptions of the relationship between effort and ability.

\section{Future Studies}

The findings of this study, and the implications they highlight, provide a foundation for a productive line of inquiry that could extend the fields of coping, 
regulation, control, and academic motivation. Suggestions for each of these areas are discussed in this section.

Coping

Additional coping families. The field of coping could benefit from more research that demonstrates the utility of the families model of ways of coping. The ways of coping used in this study have been identified as the markers of the family that is most closely associated with the competence system. Other families of ways of coping, and other constellations of ways of coping that cross families could be introduced to the models tested in this study to start unwrapping the nuanced ways in which children deal with the challenges they face in school. For example, the specific way of coping that is help-seeking was not included in this study, as it is a way of coping associated with the relatedness system. This study did not find a strong relationship between teacher support and coping; since the forms of teacher support associated with the other self-systems are included in the aggregate measure, including ways of coping from the other families associated with the other self-systems, such as help-seeking, may help detect a more influential role for teachers.

In addition, as it is likely that children differ in which self-system is most salient at a given time, and therefore more closely related to their potential for success, it may be very informative to explore other families of coping, and interactions between different ways of coping within families to identify coping profiles that promote resilience, and those that damage it. Just as control profiles for promoting 
and undermining control have been formulated and explored, there may be certain combinations of ways of coping that function in a similar manner with respect to motivation and engagement. An investigation into this possibility is warranted.

Developmentally appropriate measures. A limitation of this study was the lack of measures for coping that could differentiate between age appropriate behaviors that are functionally equivalent. This concept is at the heart of the coping families framework (Skinner et al., 2003), but was not used in the formulation or validation process of the coping measures used for this study. New studies should be undertaken to understand what each of the ways of coping in each family look like during different developmental periods, and measures constructed and validated that can capture those behaviors. Ideally, when studies of age differences like the present study are conducted in the future, measures for a series of four adjacent developmental periods should be included, to capture the whole progression of changes in coping as children move into, through, and out of the developmental periods of interest. Had the measures used in the present study been more nuanced in this way, for example, including items that aligned with cognitive problem-solving, significant findings may have been detected.

\section{Regulation}

Dynamic models. As a process that inherently encompasses iterative feedforward and feedback loops involving interactions between a person and their contexts, regulation is a dynamic, multilevel, interactive process (McClelland \& 
Cameron, 2011). Yet, the study designs and methodologies used to investigate regulatory systems are largely based on a linear perspective. Even the present study, with its emphasis on a systems perspective and attempt to analyze feedback loops, was constrained in its ability to detect significant findings by the use of linear statistical procedures. More complex modeling techniques, such as dynamical systems modeling using specialized software packages for this purpose (e.g., STELLA, Vensim) can be applied to the investigation of phenomena where multiple variables or levels of variables are acting iteratively and simultaneously on other variables. Complex modeling techniques that take into account the shapes of a variety of trajectories can better describe the network of influences at play.

Cybernetic control systems. As an analogue to human systems, the field of cybernetic control systems can offer a generic template for how regulatory processes proceed. From very simple systems to highly complex ones, the components of cybernetic systems can be translated into human factors in any regulatory process, and thus may offer an organizing framework for conceptualizing any human regulatory system that can guide future explorations of their functioning. This could make possible a means of specifying the mechanisms by which a person's internal scripting is modified through the iterative interactions with the environment that send information through the feedback loops to be stored or encoded somehow and utilized in subsequent regulatory episodes. With so many different types of regulatory 
systems, a unifying framework such as this may be key to understanding the overlap and interaction between systems that shape developmental outcomes.

Perceived Control.

Control as consequence. The current study positioned control resources as an antecedent to coping and engagement. Control may, however, also be a consequence of feedback processes. To understand the development over time of any construct, both perspectives of how the construct is an antecedent to other outcomes (the present study), and is a consequence of other processes (future studies) should be considered. The construct of perceived control has been widely studied and is well documented; however, the field of control could benefit from additional studies that attempt to locate where in the regulation feedback loop control resources change, and which take a developmental view of control in the process of systems regulation.

Control as mediator. The process model underlying the current study contains multiple possibilities for mediated relationships. Only the relationships between coping, engagement, and achievement were tested in the present study for mediational effects. Mediation models involving aspects of perceived control could also be tested to better understand the role that control plays in promoting motivated behavior in the classroom. For example, perceived control as a mediator between coping and engagement, or teacher support and coping may provide additional information about how coping influences engagement, or how interactions with teachers translate into adaptive or maladaptive behaviors. 


\section{Academic Motivation}

Different learning contexts. The present study used a composite score of grades across time in math and reading to arrive at an index of achievement as the distal outcome of interest. It is possible that motivation differs widely for different areas of study. For example, many children express dislike for the study of mathematics, but may be more enthusiastic about social studies. Exploring differential motivation by subject matter may provide insights into the antecedents, consequences and leverage points for building motivational resources. Investigation of the ebb and flow of motivational energy during the transition from one subject to the next may also provide clues as to how the feedback processes operate and on what time scale feedback is incorporated into the next cycle of feedforward effects.

As teaching methodologies and philosophies continue to evolve, scenarios that include approaches such as differentiated instruction, blended classrooms, and peer mentoring models should be considered as landscapes for further investigation. The unique environmental features of situations like these provide a rich opportunity to gain a more nuanced understanding of how external factors shape the development of a child's internal resources. By comparing the changes in the components of the model between these different types of contexts, a deeper understanding of a child's vulnerabilities and the opportunities for maximum effect of intervening actions might be identified. 
Social partners. Engagement, the equivalent to academic motivation in the present study, is conceptualized as having two components - behavioral and emotional. Regulation of behavior and emotion may be individual, yet deeply intertwined processes, and may be highly sensitive to the influences of social input. Coping, as a socially embedded process, involves processes of co-regulation as children engage in metacognitive activities with their social partners such as questioning, brainstorming, and prompting. Peer group processes likely encompass much of this activity and as such present peers as co-regulators of motivated action. Future studies should be conducted looking at the individual and combined contributions that different social partners, such as peers and parents, bring to the development of regulation processes within the child to produce active engagement. Metacognition. These types of social interactions and the metacognitive activities they encompass imply a cognitive component to engagement that is missing in the conceptualization of the construct used in the present study. More research is needed to understand the role of cognition and metacognitive processes in building and maintaining motivational energy. No measures of cognitive development were included in the present study; where children are in relation to the emergence of formal operations may also influence metacognitive abilities. Collaborative learning is a form of co-regulation, and most certainly activates the metacognitive processes that contribute to children's ability to be conscious producers of their own development. Socially based metacognitive activity and co-regulation processes are 
also prominent contributors to the process of emotion regulation, and warrant attention in future studies that seek to untangle the interaction of regulation of behavioral and emotion engagement with cognitive engagement. Through this line of inquiry a richer picture of academic motivation may emerge.

\section{Other considerations}

Group differences. The current study sought to identify group differences based on age as the developmental driver. The findings of this study, or lack thereof, could be extended by research that applies the process model to student characteristics that can be used to define group membership such as gender and ethnicity. Additionally, these same analyses should be conducted with other age groups, both younger and older, to form a more comprehensive picture of how coping develops over the entire spectrum of childhood. Moreover, these models should also be applied to less homogeneous samples of students to increase the utility and generalizability of the understandings gleaned about how personal resources develop, as well as the models and methodologies used to conduct such inquiries.

Expanded data collections. Finally, longitudinal studies of longer duration, with more frequent measurements over the course of a year or more should be conducted to better capture the micro-processes that likely occur during a coping episode. Studies encompassing a wider variety of variables and relationships, as has been discussed, should be conducted in order to identify the critical developmental periods and sources of influence that prompt old coping behaviors to fade and new, 
functionally equivalent ones to emerge. Also, comparisons of nested populations should be explored, such as differences between classes of students within a school, or between students in different schools within a district to further identify differential impacts of varying patterns of influence.

\section{Conclusion}

This study sought to bring to bear a new perspective on the question of how personal resources, particularly coping strategies and perceived control, develop over time. The results of this study provide confirmation of a general model that reflects the processes at work to facilitate adaptive coping and positive student outcomes. Only partial confirmation was obtained for the individual models of coping previously identified by this researcher; this is most likely due to methodological differences between studies rather than actual differences in developmental processes. Developmental differences were not found for any of the hypothesized relationships; however, developmental trends in both the mean level comparisons and in the trends identified by the predicted values of the regression equations did result, providing a preliminary glimpse of how coping develops and operates for students in middle childhood. It is with great hope that this inquiry is committed to the public domain so that others may build on its efforts, fostering new life and energy in the field of developmental science, and an initiation of an exciting new age of enlightenment with respect to children's coping, regulation, and motivation, particularly in the academic domain. 


\section{REFERENCES}

Aldwin, C.M., Skinner, E.A., Zimmer-Gembeck, M.J., \& Taylor, A.L. (2011). Coping and self-regulation across the life-span. In Fingerman, K.L., Berg, C.A., Smith, J., Antonucci, T.C. (Eds), Handbook of life-span development, (pp. 561-587). New York, NY, US:Springer Publishing Co.

Ames, C., \& Archer, J. (1988). Achievement goals in the classroom: Students' learning strategies and motivation processes. Journal of Educational Psychology, $80(3), 260-267$.

Andrews, G.R., \& Debus, R.L. (1978). Persistence and the causal perception of failure: Modifying cognitive attributions. Journal of Educational Psychology, 70(2), 154-166.

Barling, J. (1982). Self-determined performance standards and locus of control beliefs in children's academic performance. British Journal of Educational Psychology, $52,100-103$.

Baron, R.M., \& Kenny, D.A. (1986). The moderator-mediator variable distinction in social psychological research: Conceptual, strategic, and statistical considerations. Journal of Personality and Social Psychology, 51(6), 1173-1182.

Boggiano, A.K., Main, D.S., \& Katz, P.A. (1988). Children's preference for challenge: The role of perceived competence and control. Journal of Personality and Social Psychology, 54(1), 134-141. 
Bong, M. (2001). Between and within domain relations of academic motivation among middle and high school students: Self-efficacy, task value, and achievement goals. Journal of Educational Psychology, 93(1), 23-34.

Bouffard, T., \& Vezeau, C. (1998). The developing self-system and self-regulation of primary school children. In Ferrari, M.D., \& Sternberg, R.J. (Eds.), Selfawareness: Its nature and development (pp. 246-272). New York, NY: Guilford Press.

Bouffard, T., Boileau, L., \& Vezeau, C. (2001). Student's transition from elementary to high school and changes of the relationship between motivation and academic performance. European Journal of Psychology of Education, 16(4), 589-604.

Bouffard, T., Boisvert, M., \& Vezeau, C. (2003). The illusion of incompetence and its correlates among elementary school children and their parents. Learning and Individual Differences, 14, 31-46.

Bouffard-Bouchard, T., Parent, S., \& Larivee, S. (1991). Influence of self-efficacy on self-regulation and performance among junior and senior high-school age students. International Journal of Behavioral Development, 14(2), 153-164.

Butkowsky, I.S., \& Willows, D.M. (1980). Cognitive-motivational characteristics of children varying in reading ability: Evidence for learned helplessness in poor readers. Journal of Educational Psychology,72(3), 408-422. 
Cain, K.M., \& Dweck, C.S. (1995). The relation between motivational patterns and achievement cognitions through the elementary school years. Merrill Palmer Quarterly, 41(1), 25-52.

Causey, D.L., \& Dubow, E.F. (1992). Development of a self-report coping measure for elementary school children. Journal of Clinical Child Psychology, 21(1), 4759.

Chapin, M., \& Dyck, D.G. (1976). Persistence in children's reading behavior as a function of $\mathrm{N}$ length and attribution retraining. Journal of Abnormal Psychology, $85(5), 511-515$.

Christenson, S. L., Reschly, A. L., \& Wylie, C. (2012). Handbook of research on student engagement. New York: Springer Science.

Collins, L.M., Schafer, J.L., \& Kam, C.M. (2001). A comparison of inclusive and restrictive strategies in modern missing data procedures. Psychological Methods, $6,330-351$.

Compas, B.E. (1987). Coping with stress during childhood and adolescence. Psychological Bulletin, 101(3), 393-403.

Compas, B.E. (1991). Perceived control and coping with stress: A developmental perspective. Journal of Social Issues, 47(4), 23-34. 
Compas, B.E., Connor-Smith, J.K., Saltzman, H., Thompsen, A.H., \& Wadsworth, M.E. (2001). Coping with stress during childhood and adolescence: Problems, progress, and potential in theory and research. Psychological Bulletin, 127(1), 87127.

Connell, J.P. (1990). Context, self, and action: A motivational analysis of self-system processes across the life-span. In Cichetti, D., \& Beeghly, M. (Eds.), The self in transition: Infancy to childhood (pp.61-97). Chicago, IL: University of Chicago Press.

Connell, J.P., \& Wellborn, J.G. (1991). Competence, autonomy, and relatedness: A motivational analysis of self-system processes. In Gunnar, M.R., \& Sroufe, L.A. (Eds.), Self processes and development: The Minnesota symposia on child development, Vol. 23 (pp. 43-77).

Connell, J.P., Spencer, M.B., \& Aber, J.L. (1994). Educational risk and resilience in African-American youth: Context, self, action, and outcomes in school. Child Development, 65, 493-506.

Craske, M.L. (1985). Improving persistence through observational learning and attribution retraining. British Journal of Educational Psychology, 55, 138-147.

Deci, E.L., \& Ryan, R. (1985). Intrinsic motivation and self-determination in human behavior. New York, NY: Plenum Press. 
Demirtas, H., Freels, S.A. \& Yucel, R.M. (2008). Plausibility of multivariate normality assumption when multiply imputing non-Gaussian continuous outcomes: A simulation assessment. Journal of Statistical Computation and Simulation, 78(1), 69-84.

Diener, C.I., \& Dweck, C.S. (1978). An analysis of learned helplessness: Continuous changes in performance, strategy, and achievement cognitions following failure. Journal of Personality and Social Psychology, 36(5), 451-462.

Diener, C.I., \& Dweck, C.S. (1980). An analysis of learned helplessness: II. The process of success. Journal of Personality and Social Psychology, 39(5), 940-952.

Doron, J., Yannick, S., Boiché, J., \& Le Scanff, C. (2009). Coping with examinations: Exploring relationships between students' coping strategies, implicit theories of ability, and perceived control. British Journal of Educational Psychology, 79, $575-528$.

Dweck, C.S. (1975). The role of expectations and attributions in the alleviation of learned helplessness. Journal of Personality and Social Psychology, 31(4), 674685.

Dweck, C.S., \& Reppucci, N.D. (1973). Learned helplessness and reinforcement responsibility in children. Journal of Personality and Social Psychology, 25(1), 109-116. 
Eccles, J.S., \& Midgley, C. (1989). Stage/environment fit: Developmentally appropriate classrooms for early adolescents. In Ames, R. \& Ames, C. (Eds.), Research on motivation in education, Vol. 3 (pp. 139-181). New York, NY: Academic Press.

Eccles, J.S., Midgley, C., Wigfield, A., Buchanan, C.M., Reuman, D., Flanagan, C, \& MacIver, D. (1993). Development during adolescence: The impact of stageenvironment fit on young adolescents' experiences in schools and in families. American Psychologist, 48(2), 90-101.

Eccles, J.S., \& Wigfield, A. (2002). Motivational Beliefs, values, and goals. Annual Review of Psychology, 53(1), 109-132.

Edwards, J.R., \& Lambert, L.S. (2007). Methods for integrating moderation and mediation: A general analytical framework using moderated path analysis. Psychological Methods, 12(1), 1-22.

Enders, C.K., Baraldi, A.N., \& Cham, H. (2014). Estimating interaction effects with incomplete predictor variables. Psychological Methods, 19(1), 39-55.

Fields, L., \& Prinz, R.J. (1997). Coping and adjustment during childhood adolescence. Clinical Psychology Review, 17(8), 937-976.

Findley, M., \& Cooper, H. (1983). Locus of control and academic achievement: A literature review. Journal of Personality and Social Psychology, 44(2), 419-427. 
Fishman, E.J. (2014). With great control comes great responsibility: The relationship between perceived academic control, student responsibility, and self-regulation. British Journal of Educational Psychology, 84, 685-702.

Fowler, J.W., \& Peterson, P.L. (1981). Increasing reading persistence and altering attributional style of learned helplessness children. Journal of Educational Psychology, 73(2), 251-260.

Fredericks, J.A., Blumenfeld, P.C., \& Paris, A.H. (2004). School engagement: Potential of the concept, state of the evidence. Review of Educational Research, 74(1), 59-109.

Furrer, C.J. \& Skinner, E.A. (2003). Sense of relatedness as a factor in children's academic engagement and performance. Journal of Educational Psychology, 95(1), 148-162.

Graham, J.W. (2009). Missing data analysis: Making it work in the real world. Annual Review of Psychology, 60, 549-576.

Graham, J.W. (2012). Missing data analysis and design. New York:Springer.

Graham, J.W., \& Schafer, J.L. (1999). On the performance of multiple imputation for multivariate data with small sample size. In Hoyle, R. (Ed.), Statistical strategies for small sample research, (pp. 1-29). Thousand Oaks, CA:Sage. 
Graham, J.W., Cumsille, P.E., \& Elek-Fisk, E. (2003). Methods for handling missing data. In Schinka, J.A., \& Velicer, W.F. (Eds), Research Methods in Psychology, (pp. 87-114), Vol 2, Handbook of Psychology, Weiner, I.B., (Ed.). New York:Wiley.

Graham, J.W., Olchowski, A.E., \& Gilreath, T.D. (2007). How many imputations are really needed? Some practical clarifications of multiple imputation theory. Prevention Science, 8, 206-213.

Grant, H., \& Dweck, C. (2003). Clarifying achievement goals and their impact. Journal of Personality and Social Psychology, 85(3), 541-553.

Greene, B.A., Miller, R.B., Crowson, M., Duke, B.L., \& Akey, K.L. (2004). Predicting high school student's cognitive engagement and achievement: Contributions of classroom perceptions and motivation. Contemporary Educational Psychology, 29, 462-482.

Greene, J. (1985). Relationships among learning and attribution theory motivational variables. American Educational Research Journal, 22(1), 65-78.

Greene, T.M. (2007). Perceived control and coping: Personal resources as pathways to academic engagement. Unpublished master's thesis. Portland State University, Portland, Oregon.

Hayes, A. (2009). Beyond Baron and Kenny: Statistical mediation analysis in the new millennium. Communication Monographs, 76, 408-420. 
Hayes, A. (2013). Introduction to mediation, moderation, and conditional process analysis: A regression-based approach. New York, NY. The Guilford Press.

Hayes, A. (2015). An index and test of linear moderated mediation. Multivariate Behavioral Research, 50(1), 1-22.

James, L.R., \& Brett, J.M. (1984). Mediators, moderators, and tests for mediation. Journal of Applied Psychology, 69, 307-321.

Kalechstein, A.D, \& Nowicki Jr., S. (1997). A meta-analytic examination of the relationship between control expectancies and academic achievement: An 11-year follow-up to Findley and Cooper. Genetic, Social \& General Psychology Monographs, 123(1), 29-57.

Lerner, R. M., \& Busch-Rossnagel, N. (Eds.). (1981). Individuals as producers of their own development: A lifespan perspective. New York, Academic Press.

Lopez, D. (1999). Social cognitive influences on self-regulated learning: The impact of action-control beliefs and academic goals on achievement-related outcomes. Learning \& Individual Differences, 11(3), 301-320.

Linnenbrink E.A. (2005). The dilemma of performance-approach goals: The use of multiple goal contexts to promote students' motivation and learning. Journal of Educational Psychology, 97(2), 197-213.

MacIver, D.J., Stipek, D.J., \& Daniels, D.H. (1991). Explaining within-semester changes in student effort in junior high school and senior high school courses. Journal of Educational Psychology, 83(2), 201-211. 
MacKinnon, D.P., Lockwood, C.M., Hoffman, J.M., West, S.G., \& Sheets, V. (2002). A comparison of methods to test mediation and other intervening variable effects. Psychological Methods, 7, 83-104.

Mantzicopoulos, P. (1990). Coping with failure: Characteristics of students employing successful and unsuccessful coping strategies. Psychology in the Schools, 27(2), $138-143$.

Mantzicopoulos, P. (1997). How do children cope with school failure? A study of social/emotional factors related to children's coping strategies. Psychology in the Schools, 34(3), 229-237.

McClelland, M.M., \& Cameron, C.E. (2011). Self-regulation and academic achievement in elementary school children. New Directions in Child and Adolescent Development, 133, 29-44.

Medway, F.J., \& Venino, G.R. (1982). The effects of effort feedback and performance patterns on children's attributions and task persistence. Contemporary Educational Psychology, 7, 26-34.

Midgley, C., \& Urdan, T. (1995). Predictors of middle school students' use of selfhandicapping strategies. Journal of Early Adolescence, 15(4), 389-411.

Miller, R.B., Greene, B.A., Montalvo, G.P., Ravindran, B., \& Nichols, J.D. (1996). Engagement in academic work: The role of learning goals, future consequences, pleasing others, and perceived ability. Contemporary Educational Psychology, 21, $388-422$. 
Muller, D., Judd, C.M., Yzerbyt, V.Y. (2005). When moderation is mediated and mediation is moderated. Journal of Personality and Social Psychology, 89(6), 852863.

Musher-Eizenman, D. R., Nesselroade, J.R., \& Schmitz, B. (2002). Perceived control and academic performance: A comparison of high- and low-performing children on within-person change patterns. International Journal of Behavioral Development, 26(6), 540-547.

Nolen-Hoksema, S., Girgus, J.S., \& Seligman, M.E.P. (1986). Learned helplessness in children: A longitudinal study of depression, achievement, and explanatory style. Journal of Personality and Social Psychology, 51(2), 435-442.

Nowicki, Jr., S., \& Strickland, B. (1973). A locus of control scale for children. Journal of Consulting and Clinical Psychology, 40(1), 148-154.

Obach, M. (2003). A longitudinal-sequential study of perceived academic competence and motivational beliefs for learning among children in middle school. Educational Psychology, 23(3), 323-338.

Oettingen, G., Little, T.D., Lindenberger, U., \& Baltes, P.B. (1994). Causality, agency, and control beliefs in East versus West Berlin children: A natural experiment on the role of context. Journal of Personality and Social Psychology, 66(3), 579-595.

Patrick, B.C., Skinner, E.A., \& Connell, J.P. (1993). What motivates children's behavior and emotion? Joint effects of perceived control and autonomy in the academic domain. Journal of Personality and Social Psychology, 65(4), 781-791. 
Paunesku, D., Walton, G.M., Romero, C., Smith, E.N., Yeager, D.S., \& Dweck, C.S. (2015). Mind-set interventions are a scalable treatment for academic underachievement. Psychological Science, 26(6), 784-793.

Perry, R., Hladkyj, S., Pekrun, R., Clifton, R., \& Chipperfield, J. (2005). Perceived academic control and failure in college students: A three-year study of scholastic attainment. Research in Higher Education, 46(5), 535-569.

Perry, R.P., \& Magnusson, J. (1988). Causal attributions and perceived performance: Consequences for college students' achievement and perceived control in different instructional conditions. Journal of Educational Psychology, 81(2), 164-172.

Peugh, J.L., \& Enders, C.K (2004). Missing data in educational research: A review of reporting practices and suggestions for improvement. Review of Educational Research, 74(4), 525-556.

Phillips, D. (1984). The illusion of incompetence among academically competent children. Child Development, 55, 2000-2016.

Pokay, P., \& Blumenfeld, P.C. (1990). Predicting achievement early and late in the semester: The role of motivation and use of learning strategies. Journal of Educational Psychology,82(1), 41-50.

Pomeranzt, E.M., \& Saxon, J.L. (2001). Conceptions of ability as stable and selfevaluative processes: A longitudinal examination. Child Development, 72(1), 152173. 
Prawat, R. (1976). Mapping the affective domain in young adolescents. Journal of Educational Psychology,68(5), 566-572.

Prawat, R.S., Grissom, S., \& Parish, T. (1979). Affective development in children, grades 3 through 12. The Journal of Genetic Psychology, 135, 37-49.

Preacher, K. J., Rucker, D. D., \& Hayes, A. F. (2007). Assessing moderated mediation hypotheses: Theory, methods, and prescriptions. Multivariate Behavioral Research, 42, 185-227.

Roeser, R. W., Midgley, C., \& Urdan, T. C. (1996). Perceptions of the school psychological environment and early adolescents' psychological and behavioral functioning in school: The mediating role of goals and belonging. Journal of Educational Psychology, 88(3), 408-422.

Rubin, D.B. (1987). Multiple imputation for nonresponse in surveys. New York:Wiley.

Schafer, J.L., \& Graham, J.W. (2002). Missing data: Our view of the state of the art. Psychological Methods, 7(2), 147-177.

Schneewind, K. A. (1995). Impact of family processes on control beliefs. In Bandura, A. (Ed.), Self-efficacy in changing societies (pp. 114-148). New York, NY, US: Cambridge University Press.

Shrout, P.E., \& Bolger, N. (2002). Mediation in experimental and non-experimental studies: New procedures and recommendations. Psychological Methods, 7, 422445. 
Sink, C.A., Barnett, J.E., \& Hixon, J. (1991). Self-regulated learning and achievement by middle-school children. Psychological Reports, 69, 979-989.

Skinner, E. A. (1990). Age differences in the dimensions of perceived control during middle childhood: Implications for developmental conceptualizations and research. Child Development, 61, 1882-1890.

Skinner, E.A. (1992). Perceived control: Motivation, coping, and development. In Schwarzer, R. (Ed.), Self-efficacy: Thought control of action. (pp.91-106). Washington, D.C., US: Hemisphere Publishing Corp.

Skinner, E.A., (1996). A guide to constructs of control. Journal of Personality and Social Psychology, 71(3), 549-570.

Skinner, E.A., (1999). Action regulation, coping, and development. In Brandtstädter, J., \& Lerner, R.M., (Eds.), Action \& self-development: Theory and research through the life-span, (pp.465-503). Thousand Oaks, CA, US: Sage Publications, Inc.

Skinner, E.A., \& Belmont, M.J. (1993). Motivation in the classroom: Reciprocal effects of teacher behavior and student engagement across the school year. Journal of Educational Psychology, 85(4), 571-581.

Skinner, E. A., \& Pitzer, J. (2012). Developmental dynamics of engagement, coping, and everyday resilience. In S. Christenson, A. Reschly, \& C. Wylie (Eds.), Handbook of research on student engagement (pp. 21-45). New York: Springer Science. 
Skinner, E.A., \& Wellborn, J.G. (1994). Coping during childhood and adolescence: A motivational perspective. In Featherman, D.L., Lerner, R.M., \& Perlmutter, M. (Eds.), Life-span development and behavior (pp. 91-133). Hillsdale, NJ: Lawrence Erlbaum Associates.

Skinner, E.A., \& Wellborn, J.G. (1997). Children's coping in the academic domain. In Wolchik, S., \& Sandler, I.N. (Eds.), Handbook of children's coping: Linking theory and intervention (pp. 387-422). New York, NY: Plenum Press.

Skinner, E.A., \& Zimmer-Gembeck, M.J. (2007). The development of coping. Annual Review of Psychology, 58, 119-144.

Skinner, E.A., \& Zimmer-Gembeck, M.J. (2009). Challenges to the developmental study of coping. New Directions for Child and Adolescent Development, 124, 517.

Skinner, E.A., \& Zimmer-Gembeck, M.J. (2011). Perceived control and the development of coping. In Folkman, S (Ed.), The Oxford handbook of stress, health, and coping (pp. 35-59). New York, NY: Oxford University Press.

Skinner, E.A., Chapman, M., \& Baltes, P.B. (1988a). Control, means-ends, and agency beliefs: A new conceptualization and its measurement during childhood. Journal of Personality and Social Psychology, 54(1), 117-133.

Skinner, E.A., Edge, K., Altman, J., \& Sherwood, H. (2003). Searching for the structure of coping: A review and critique of category systems for classifying ways of coping. Psychological Bulletin, 129(2), 216-269. 
Skinner, E., Furrer, C., Marchand, G., \& Kindermann, T. (2008). Engagement and disaffection in the classroom: Part of a larger motivational dynamic. Journal of Educational Psychology, 100(4), 765-781.

Skinner, E.A., Kindermann, T.A., \& Furrer, C.J. (2009). A motivational perspective on engagement and disaffection. Educational and Psychological Measurement, $69(3), 493-525$.

Skinner, E.A., Wellborn, J.G., \& Connell, J.P. (1990). What it takes to do well in school and whether I've got it: A process model of perceived control and children's engagement and achievement in school. Journal of Educational Psychology, 82(1), 22-32.

Skinner, E.A., Zimmer-Gembeck, M. J., \& Connell, J.P. (1998). Individual differences and the development of perceived control. Monographs of the Society for Research in Child Development, 254(63), Nos. 2-3.

Spinath, B., \& Spinath, F.M. (2005). Longitudinal analysis of the link between learning motivation and competence beliefs among elementary school children. Learning and Instruction, 15, 87-102.

Stipek, D.J., \& Mason, T.C. (1987). Attributions, emotions, and behavior in the elementary school classroom, 22(2), 1-5.

Stipek, D.J., \& Weisz, J.R. (1981). Perceived control and academic achievement. Review of Educational Research, 51(1), 101-137. 
Sukkyung You, Sehee Hong \& Hsiu-zu Ho (2011). Longitudinal effects of perceived control on academic achievement. The Journal of Educational Research, 104(4), 253-266.

Tabachnick, B.G., \& Fidell, L.S. (1996). Using multivariate statistics. New York: NY: HarperCollins College Publishers.

Tanner, M.A., \& Wong, W.H. (1987). The calculation of posterior distributions by data augmentation (with discussion). Journal of the American Statistical Association, 82, 528-550.

Urdan, T., Midgley, C., \& Anderman, E.M. (1998). The role of classroom goal structure in students' use of self-handicapping strategies. American Educational Research Journal, 35(1), 101-122.

Von Hippel, P.T. (2009). How to impute interactions, squares, and other transformed variables. Sociological Methodology, 39, 265-291.

Walls, T.A., \& Little, T.D. (2005). Relations among personal agency, motivation, and school adjustment in early adolescence. Journal of Educational Psychology, 97(1), 23-31.

Weiner, B. (1985a). An attributional theory of achievement motivation and emotion. Psychological Review, 92(4), 548-573.

Wigfield, A., \& Eccles, J.S. (2002). Motivational beliefs, values, and goals. Annual Review of Psychology, 53, 109-132. 
Wigfield, A., Eccles, J, Schiefele, U., Roeser, R. \& Davis-Kean, P. (2006). Development of achievement motivation. In W. Damon (Series Ed.) \& N. Eisenberg (Volume Ed.), Handbook of Child Psychology, $6^{\text {th }}$ Ed. Vol. 3, Social, Emotional, and Personality Development (pp. 933-1002). New York: John Wiley. Wigfield, A., Eccles, J.S., Fredricks, J.A., Simpkins, S., Roeser, R.W., \& Schiefele, U. (2015). Development of achievement motivation and engagement. In M. E. Lamb \& R.E. Lerner (Eds.), Handbook of Child Psychology and Developmental Science, $7^{\text {th }}$ Ed. Vol. 3, Socioemotional Processes (pp. 657-700). Hoboken, NJ: John Wiley \& Sons, Inc.

Wolchik, S. \& Sandler, I.N. (1997). Handbook of children's coping: Linking theory and intervention. New York, NY: Plenum Press.

Wolters, C. (2004). Advancing achievement goal theory: Using goal structures and goal orientations to predict students' motivation, cognition, and achievement. Journal of Educational Psychology, 96(2), 236-250.

Wong, E.H., Weist, D.J., \& Cusick, L.B. (2002). Perceptions of autonomy support, parent attachment, competence and self-worth as predictors of motivational orientation and academic achievement: An examination of sixth- and ninth-grade regular education students. Adolescence, 37(146), 255-266. 
Yeager, D.S. \& Dweck, C.S. (2012). Mindsets that promote resilience: When students believe that personal characteristics can be developed. Educational Psychologist, $47,1-13$.

Zimmer-Gembeck, M. J., \& Skinner, E. A. (2011). The development of coping across childhood and adolescence: An integrative review and critique of research. International Journal of Behavioral Development 35(1), 1-17. 


\section{APPENDIX A}

\section{Questionnaire items and item numbers}

Student Perceived Control Questionnaire

\begin{tabular}{|c|c|}
\hline $\begin{array}{l}\text { Control Beliefs } \\
\text { Items }\end{array}$ & Item Number \\
\hline $\begin{array}{l}\text { 1. If I decide to learn something hard, I can. } \\
\text { 2. I can do well in school if I want to. } \\
\text { 3. I can get good grades in school. } \\
\text { 4. I can't get good grades, no matter what I do. } \\
\text { 5. I can't stop myself from doing poorly in school. } \\
\text { 6. I can't do well in school, even if I want to. }\end{array}$ & $\begin{array}{l}\text { ASCNP01x } \\
\text { ASCNP02x } \\
\text { ASCNP03x } \\
\text { ASCNN01x } \\
\text { ASCNN02x } \\
\text { ASCNN03x }\end{array}$ \\
\hline \multicolumn{2}{|l|}{$\begin{array}{l}\text { Strategy Beliefs-Effort } \\
\text { Items }\end{array}$} \\
\hline $\begin{array}{l}\text { 1. If I want to do well on my schoolwork, I just need to try hard. } \\
\text { 2. The best way for me to get good grades is to work hard. } \\
\text { 3. If I don't do well in school, it's because I didn't work hard } \\
\text { enough. } \\
\text { 4. If I get bad grades, it's because I didn't try hard enough. } \\
\text { 5. If I don't do well on my schoolwork, it's because I didn't try } \\
\text { hard enough. }\end{array}$ & $\begin{array}{l}\text { ASMEP02 } x \\
\text { ASMEP03x } \\
\text { ASMEN01x } \\
\text { ASMEN02x } \\
\text { ASMEN03x }\end{array}$ \\
\hline \multicolumn{2}{|l|}{$\begin{array}{l}\text { Strategy Beliefs-Ability } \\
\text { Items }\end{array}$} \\
\hline $\begin{array}{l}\text { 1. I have to be smart to get good grades in school. } \\
\text { 2. If I want to do well in school, I have to be smart. } \\
\text { 3. If I'm not smart, I won't get good grades. } \\
\text { 4. If I'm not smart in a school subject, I won't do well at it. }\end{array}$ & $\begin{array}{l}\text { ASMAP01x } \\
\text { ASMAP03x } \\
\text { ASMAN01x } \\
\text { ASMAN03x }\end{array}$ \\
\hline \multicolumn{2}{|l|}{$\begin{array}{l}\text { Strategy Beliefs-Powerful Others } \\
\text { Items }\end{array}$} \\
\hline $\begin{array}{l}\text { 1. To well in school, I just have to get the teacher to like me. } \\
\text { 2. If I want to get good grades in a subject, I have to get along with } \\
\text { my teacher. } \\
\text { 3. I won't do well in school if my teacher doesn't like me. } \\
\text { 4. If I get bad grades, it's because I don't get along with my } \\
\text { teacher. }\end{array}$ & $\begin{array}{l}\text { ASMOP01x } \\
\text { ASMOP03x } \\
\text { ASMON01x } \\
\text { ASMON03x }\end{array}$ \\
\hline
\end{tabular}




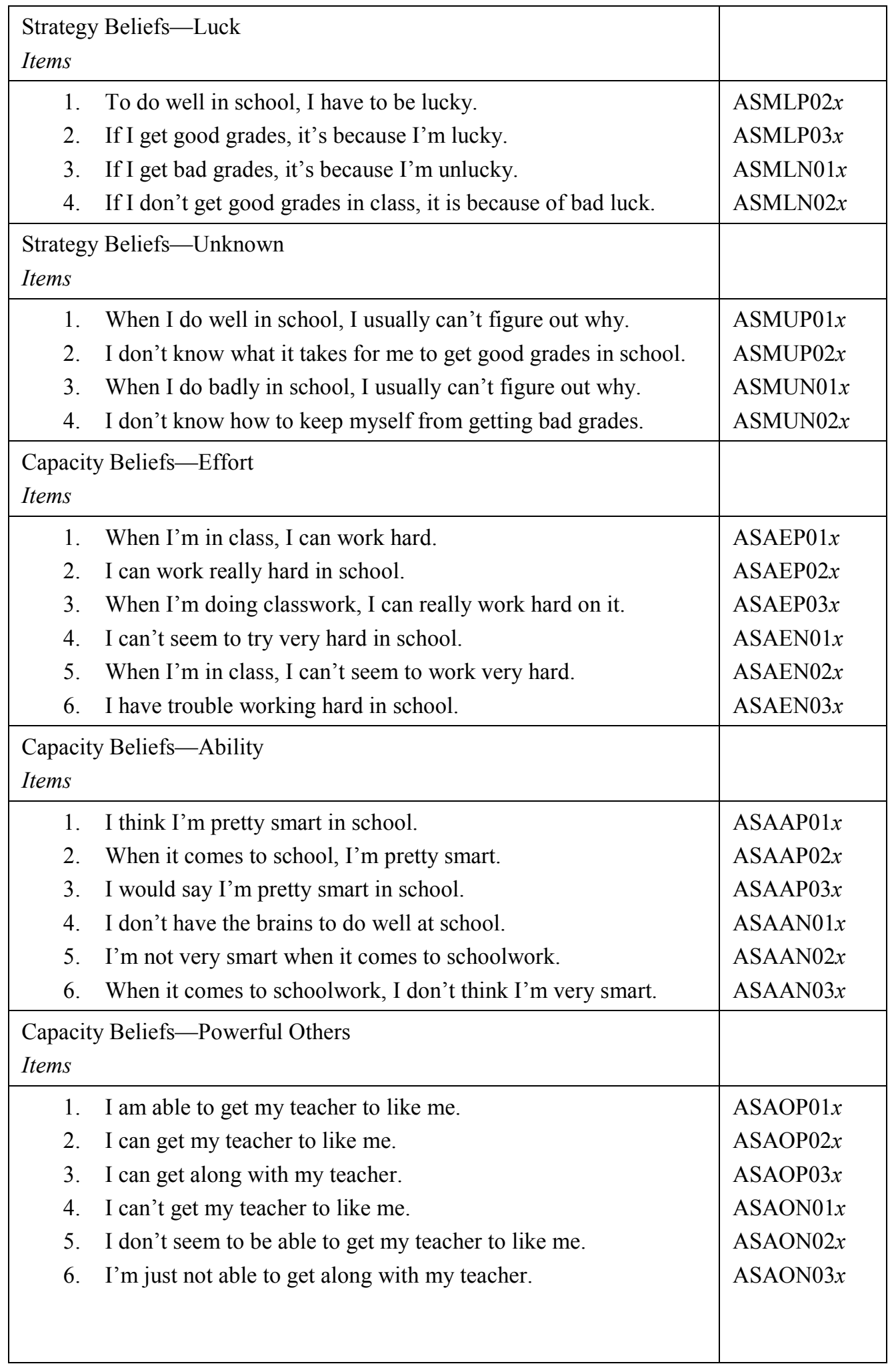




\begin{tabular}{|c|l|}
\hline $\begin{array}{l}\text { Capacity Beliefs-Luck } \\
\text { Items }\end{array}$ & \\
\hline 1. I am lucky in school. & ASALP01x \\
$2 . \quad$ I'm pretty lucky when it comes to getting grades. & ASALP02x \\
3. As far as doing well in school goes, I'm pretty lucky. & ASALP03x \\
4. I am unlucky when it comes to schoolwork. & ASALN01x \\
$5 . \quad$ When it comes to grades, I'm unlucky. & ASALN02x \\
$6 . \quad$ I am unlucky at my schoolwork. & ASALN03x \\
\hline
\end{tabular}

Student Coping Questionnaire

Problem-Solving
Items
When something bad happens to me in school, like not doing well on a
test or not being able to answer an important question,

1. I try to figure out what I did wrong so that it won't happen again.

2. I try to see what I did wrong.

$\operatorname{ASCST} 52 x$

3. I think about some way to keep this from happening again.

$\operatorname{ASCST} 53 x$

4. I try to figure out how to do better next time.

5. I think of some things that will help me next time. $\operatorname{ASCST} 54 x$ $\operatorname{ASCST} 55 x$

Information-Seeking

Items

When I have trouble with a subject in school,

1. I ask for some help with understanding the material.

ASCHS51x

2. I get some help to understand the material better.

ASCHS $52 x$

3. I ask the teacher to go over it with me.

ASCHS $53 x$

4. I ask the teacher to explain what I didn't understand.

ASCHS $54 x$

5. I get some help on the parts I didn't understand.

ASCHS55x 


\begin{tabular}{|c|c|}
\hline $\begin{array}{l}\text { Escape } \\
\text { Items }\end{array}$ & \\
\hline $\begin{array}{l}\text { 1. When I have a hard question or problem in class, I don't even } \\
\text { try. } \\
\text { 2. When I come to a problem that I can't solve right away, I just } \\
\text { give up. } \\
\text { 3. If a problem is really hard, I just quit working on it. } \\
\text { 4. If I don't understand something right away, I stop trying. }\end{array}$ & $\begin{array}{l}\text { ASRAN01x } \\
\text { ASRAN02x } \\
\text { ASRAN03x } \\
\text { ASRAN53x }\end{array}$ \\
\hline $\begin{array}{l}\text { Confusion } \\
\text { Items }\end{array}$ & \\
\hline $\begin{array}{l}\text { When I run into a problem on an important test, } \\
\text { 1. I'm not sure what to do next. } \\
\text { 2. I can't remember what to do. } \\
\text { 3. My mind goes blank. } \\
\text { 4. I get all confused. } \\
\text { 5. It's difficult for me to think. }\end{array}$ & $\begin{array}{l}\text { ASCCF51x } \\
\text { ASCCF52x } \\
\text { ASCCF53x } \\
\text { ASCCF54x } \\
\text { ASCCF55x }\end{array}$ \\
\hline
\end{tabular}

Student Engagement Questionnaire

\begin{tabular}{|c|l|}
\hline $\begin{array}{l}\text { Behavioral Engagement } \\
\text { Items }\end{array}$ & Item Number \\
\hline 1. I participate when we discuss new material. & ASIAP32x \\
2. I work hard when we start something new in class. \\
3. The first time my teacher talks about a new topic, I listen very \\
$\quad$ carefully. & ASIAP33x \\
A. When we start something new, I practically fall asleep. & \\
5. My mind wanders when my teacher starts a new topic. \\
6. I never seem to pay attention when we begin a new subject. & ASIAN31x \\
7. I try very hard in school. & ASIAN36x $x$ \\
8. I participate in class discussions. & ASOAP31x \\
9. When I am in class, I just act like I'm working. & ASOAP35x \\
10. In class, I try to do just enough to get by. & ASOAN03x \\
& ASOAN33x \\
\hline
\end{tabular}




\begin{tabular}{|l|l|}
\hline Emotional Engagement & \\
Items & \\
\hline $\begin{array}{l}\text { When we start something new in school, } \\
\text { 1. I feel interested. }\end{array}$ & ASIEP04x \\
2. I feel worried. & ASIEN04x \\
When my teacher first explains new material, & \\
3. I feel relaxed. & ASIEP32 $x$ \\
4. I feel bored. & ASIEN05x \\
When I'm working on my classwork, & \\
5. I feel relaxed. & ASOEP31x \\
6. I feel involved. & ASOEP32x \\
7. I feel nervous. & ASOEN31x \\
8. I feel mad. & ASOEN51x \\
When I'm doing my work in class, & \\
9. I feel worried. & ASOEN04x \\
10. I feel bored. & ASOEN32x \\
When I'm in class, & \\
11. I feel good. & ASEEP02x \\
12. I feel sad. & ASEEN31x \\
When I'm in school, & \\
13. I feel happy. & ASEEP32x \\
14. I feel bad. & ASEEN33x \\
15. I feel terrible. & ASEEN34x \\
\hline
\end{tabular}

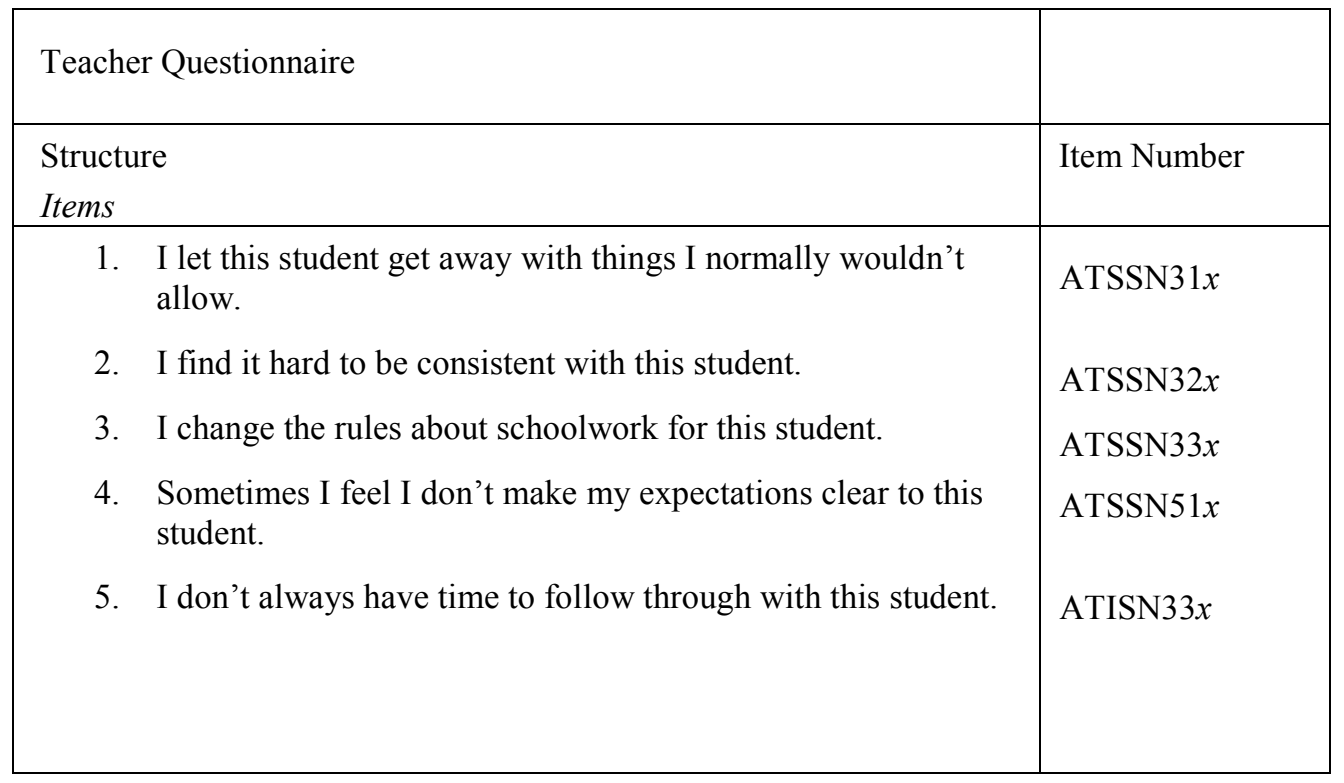




\begin{tabular}{|c|c|c|}
\hline \multirow{2}{*}{\multicolumn{2}{|c|}{$\begin{array}{l}\text { 6. I consistently apply consequences if this student doesn't meet } \\
\text { the expectations. } \\
\text { 7. I tell this student of the consequences if the expectations are } \\
\text { not met }\end{array}$}} & \multirow{2}{*}{$\begin{array}{l}\text { ATSSP } 31 x \\
\text { ATSSP } 33 x\end{array}$} \\
\hline & & \\
\hline 8. & $\begin{array}{l}\text { I try to be clear with this student about what I expect of } \\
\mathrm{him} / \text { her in class. }\end{array}$ & $\operatorname{ATSSP} 34 x$ \\
\hline \multicolumn{2}{|c|}{$\begin{array}{l}\text { Involvement } \\
\text { Items }\end{array}$} & \\
\hline & I enjoy the time I spend with this student. & ATISP31x \\
\hline & $\begin{array}{l}\text { When this student does not do as well as s/he can, I can make } \\
\text { time to help him/her find ways to do better. }\end{array}$ & $\operatorname{ATISP} 32 x$ \\
\hline & I know a lot about what goes on for this student. & ATISP $33 x$ \\
\hline & This student is easy to like. & $\operatorname{ATISP} 34 x$ \\
\hline & I know this student well. & $\operatorname{ATISP} 51 x$ \\
\hline & I spend time with this student. & ATISP $52 x$ \\
\hline 7. & I talk with this student. & $\operatorname{ATISP} 53 x$ \\
\hline & This student can count on me to be there for him/her. & ATISP54x \\
\hline & I can always find time for this student & ATISP55x \\
\hline & Teaching this student isn't very enjoyable for me. & ATISN31 $x$ \\
\hline & $\begin{array}{l}\text { This student seems to need more time than I have to give } \\
\text { him/her. }\end{array}$ & ATISN34x \\
\hline & This student is difficult to like. & ATISN $35 x$ \\
\hline & $\begin{array}{l}\text { I don't know very much about what goes on for this student } \\
\text { outside of school. }\end{array}$ & ATISN36x \\
\hline & I don't understand this student very well. & ATISN51x \\
\hline & $\begin{array}{l}\text { Sometimes I feel like I can't be there for this student when } \\
\text { s/he needs me. }\end{array}$ & ATISN $52 x$ \\
\hline & I can't always be available for this student. & ATISN53x \\
\hline
\end{tabular}




\begin{tabular}{|c|c|}
\hline $\begin{array}{l}\text { Autonomy Support } \\
\text { Items }\end{array}$ & \\
\hline $\begin{array}{l}\text { 1. I try to give this student a lot of choices about classroom } \\
\text { assignments. }\end{array}$ & ATYSP $31 x$ \\
\hline $\begin{array}{l}\text { 2. I let this student make a lot of his/her own decisions } \\
\text { regarding schoolwork. }\end{array}$ & ATYSP33x \\
\hline $\begin{array}{l}\text { 3. I encourage this student to work out problems in his / her own } \\
\text { way. }\end{array}$ & \\
\hline $\begin{array}{l}\text { 4. I review this student's work before he / she goes on to a new } \\
\text { assignment. }\end{array}$ & ATYSP51x \\
\hline 5. I let this student do classwork at his or her / own pace. & $\operatorname{ATYSP} 52 x$ \\
\hline $\begin{array}{l}\text { 6. My general approach with this student is to give him/her as } \\
\text { few choices as possible. }\end{array}$ & ATYSP53x \\
\hline $\begin{array}{l}\text { 7. I can't afford to let this student decide too many things about } \\
\text { schoolwork for him/herself. }\end{array}$ & ATYSN31x \\
\hline $\begin{array}{l}\text { 8. I have to lead this student through his/her schoolwork step by } \\
\text { step. }\end{array}$ & $\operatorname{ATYSN} 32 x$ \\
\hline $\begin{array}{l}\text { 9. When it comes to assignments, I'm always having to tell this } \\
\text { student what to do. }\end{array}$ & $\operatorname{ATYSN} 34 x$ \\
\hline $\begin{array}{l}\text { 10. I find myself telling this student every step to make when it } \\
\text { comes to schoolwork. }\end{array}$ & ATYSN36x \\
\hline 11. It's better not to give too many choices to this student. & ATYSN37x \\
\hline 12. I can't let this student do things his / her own way. & ATYSN51x \\
\hline 13. I check this student's work before teaching new material. & ATYSN $52 x$ \\
\hline 14. It's hard to know when this student is ready to move on. & $\operatorname{ATYSN} 53 x$ \\
\hline & ATYSN54x \\
\hline
\end{tabular}


Appendix B 396

\section{APPENDIX B}

Formulas for computing aggregate control constructs

\section{Control beliefs}

positive events negative events total

\section{Strategy beliefs}

Effort positive events negative events total

Ability positive events negative events total

Powerful Others positive events negative events total

Luck

positive events negative events total

Unknown positive events negative events total

\section{Capacity beliefs}

Effort positive events negative events total

Ability positive events negative events total

Powerful Others positive events negative events total

Luck

positive events negative events total

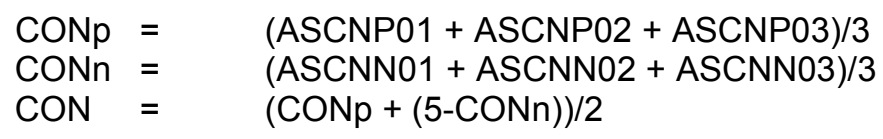

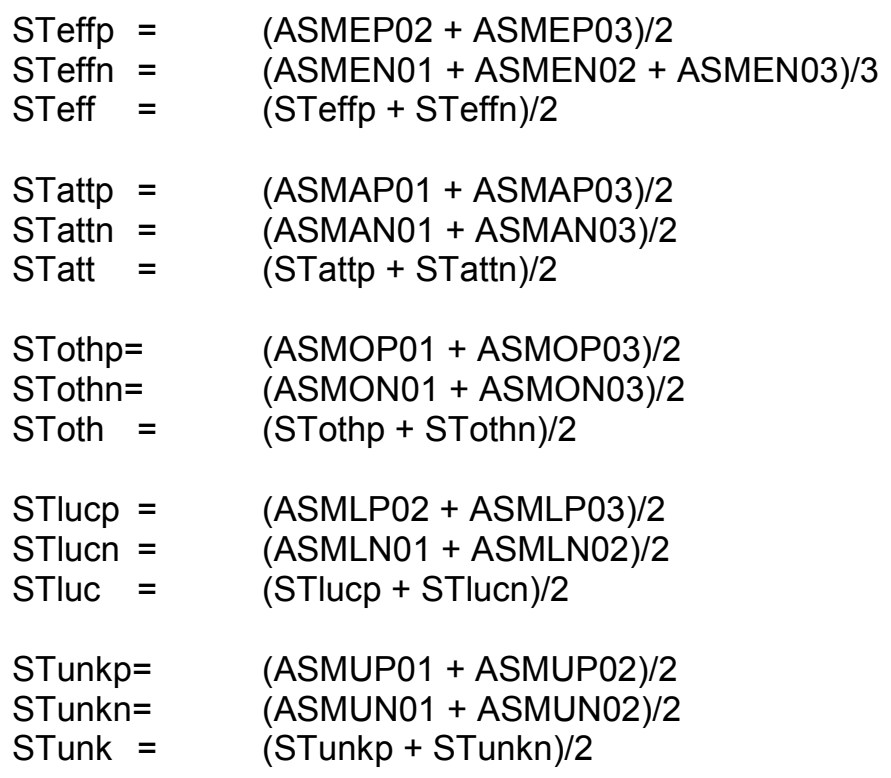

STeffp $=\quad($ ASMEP02 + ASMEP03 $) / 2$

STeffn $=\quad($ ASMEN01 + ASMEN02 + ASMEN03 $) / 3$

STattp $=\quad($ ASMAP01 + ASMAP03 $) / 2$

STattn $=\quad($ ASMAN01 + ASMAN03 $) / 2$

STatt $=($ STattp + STattn $) / 2$

STothp $=\quad($ ASMOP01 + ASMOP03 $) / 2$

STothn=

$($ ASMON01 + ASMON03)/2

STlucp $=$

(ASMLP02 + ASMLP03)/2

$($ ASMLN01 + ASMLN02)/2

STlucn $=\quad($ STlucp + STlucn $) / 2$

STunkp $=$

STunk =

ASMUN01 + ASMUN02)/2

(STunkp + STunkn)/2

$$
\begin{aligned}
& \text { CPeffp }=\quad(\text { ASAEP01 }+ \text { ASAEP02 }+ \text { ASAEP03 }) / 3 \\
& \text { CPeffn }=\quad(\text { ASAEN01 }+ \text { ASAEN02 }+ \text { ASAEN03 }) / 3 \\
& \text { CPeff }=(\text { CPeffp }+(5-\text { CPeffn }) / 2 \\
& \text { CPattp }=\quad(\text { ASAAP01 }+ \text { ASAAP02 }+ \text { ASAAP03 }) / 3 \\
& \text { CPattn }=\quad(\text { ASAAN01 }+ \text { ASAAN02 }+ \text { ASAAN03 }) / 3 \\
& \text { CPatt }=(\text { CPattp }+(5-\text { CPattn })) / 2 \\
& \text { CPothp }=\quad(\text { ASAOP01 }+ \text { ASAOP02 }+ \text { ASAOP03 }) / 3 \\
& \text { CPothn }=\quad(\text { ASAON01 }+ \text { ASAON02 }+ \text { ASAON03 }) / 3 \\
& \text { CPoth }=\quad(\text { CPothp }+(5-\text { CPothn })) / 2 \\
& \text { CPlucp }=\quad(\text { ASALP01 }+ \text { ASALP02 }+ \text { ASALP03 }) / 3 \\
& \text { CPlucn }=\quad(\text { ASALN01 }+ \text { ASALN02 }+ \text { ASALN03 }) / 3 \\
& \text { CPluc }=(\text { CPlucp }+(5-\text { CPlucn })) / 2
\end{aligned}
$$




\section{$\underline{\text { Resultant Scales }}$}

$\begin{array}{llll}\text { Scale Name } & \text { Scale Label } & \text { Range } & \text { Higher Scores Indicate: } \\ \begin{array}{llll}\text { 1. CON } & \text { Control beliefs } & (1-4) & \text { more control } \\ \text { 2. STeff } & \begin{array}{c}\text { Strategy beliefs } \\ \text { for effort }\end{array} & (1-4) & \text { effort is more effective } \\ \text { 3. STatt } & \begin{array}{c}\text { Strategy beliefs } \\ \text { for ability }\end{array} & (1-4) & \text { ability is more effective } \\ \text { 4. SToth } & \begin{array}{c}\text { Strategy beliefs } \\ \text { for powerful others }\end{array} & (1-4) & \text { powerful others are more effective } \\ \text { 5. STluc } & \begin{array}{c}\text { Strategy beliefs } \\ \text { for luck }\end{array} & (1-4) & \text { luck is more effective } \\ \text { 6. STunk } & \begin{array}{c}\text { Strategy beliefs } \\ \text { for unknown factors }\end{array} & (1-4) & \text { less is known about causes } \\ \text { 7. CPeff } & \begin{array}{c}\text { Capacity beliefs } \\ \text { for effort }\end{array} & (1-4) & \text { effort is more accessible } \\ \text { 8. CPatt } & \begin{array}{c}\text { Capacity beliefs } \\ \text { for ability }\end{array} & (1-4) & \text { ability is more accessible } \\ \text { 9. CPoth } & \begin{array}{c}\text { Capacity beliefs } \\ \text { for powerful others }\end{array} & (1-4) & \text { powerful others are more accessible } \\ \text { 10. CPluc } & \begin{array}{c}\text { Capacity beliefs } \\ \text { for luck }\end{array} & (1-4) & \text { luck is more accessible }\end{array}\end{array}$

\section{Interaction Scores for the Combination of Strategy and Capacity Beliefs}

$\begin{array}{lll}\text { Effort } & \text { INTeff }= & \text { STeff } \times \text { CPeff } \\ \text { Attributes (Ability) } & \text { INTatt }= & \text { (5-STatt) } \times \text { CPatt } \\ \text { Powerful Others } & \text { INToth }= & \text { SToth } \times \text { (5-CPoth) } \\ \text { Luck } & \text { INTluc }= & \text { STluc } \times \text { (5-CPluc) }\end{array}$

\section{Cumulative Effects on Motivation and Performance}

Total

$$
\begin{aligned}
& \text { Strategy Beliefs } \quad \text { Totstrat }=((5-S T e f f)+(5-S T a t t)+\text { SToth }+ \text { STluc }+ \text { STunk }) / 5 \\
& \text { Total } \\
& \text { Capacity Beliefs } \quad \text { Totcap }=(\text { CPeff }+ \text { CPatt }+ \text { CPoth }+ \text { CPluc }) / 5 \\
& \text { Promote } \quad \text { Promote }=(\text { CON x 4) }+(\text { STeff } \times \text { CPeffp })+((5-\text { STatt }) \times \text { CPattp })+ \\
& (\text { CPothp } \times 4)+(\text { CPlucp } \times 4) \\
& \text { Undermine } \quad \text { Undermine }=(\text { STunk } \times 4)+(\text { CPeffn } \times 4)+(\text { CPattn } \times 4)+ \\
& \text { (SToth } x \text { CPothn })+(\text { STluc } \times \text { CPlucn) }
\end{aligned}
$$




\section{APPENDIX C}

Results Graphs for All Regression Equations, by Research Question

Research Question 1: Coping - Engagement - Achievement

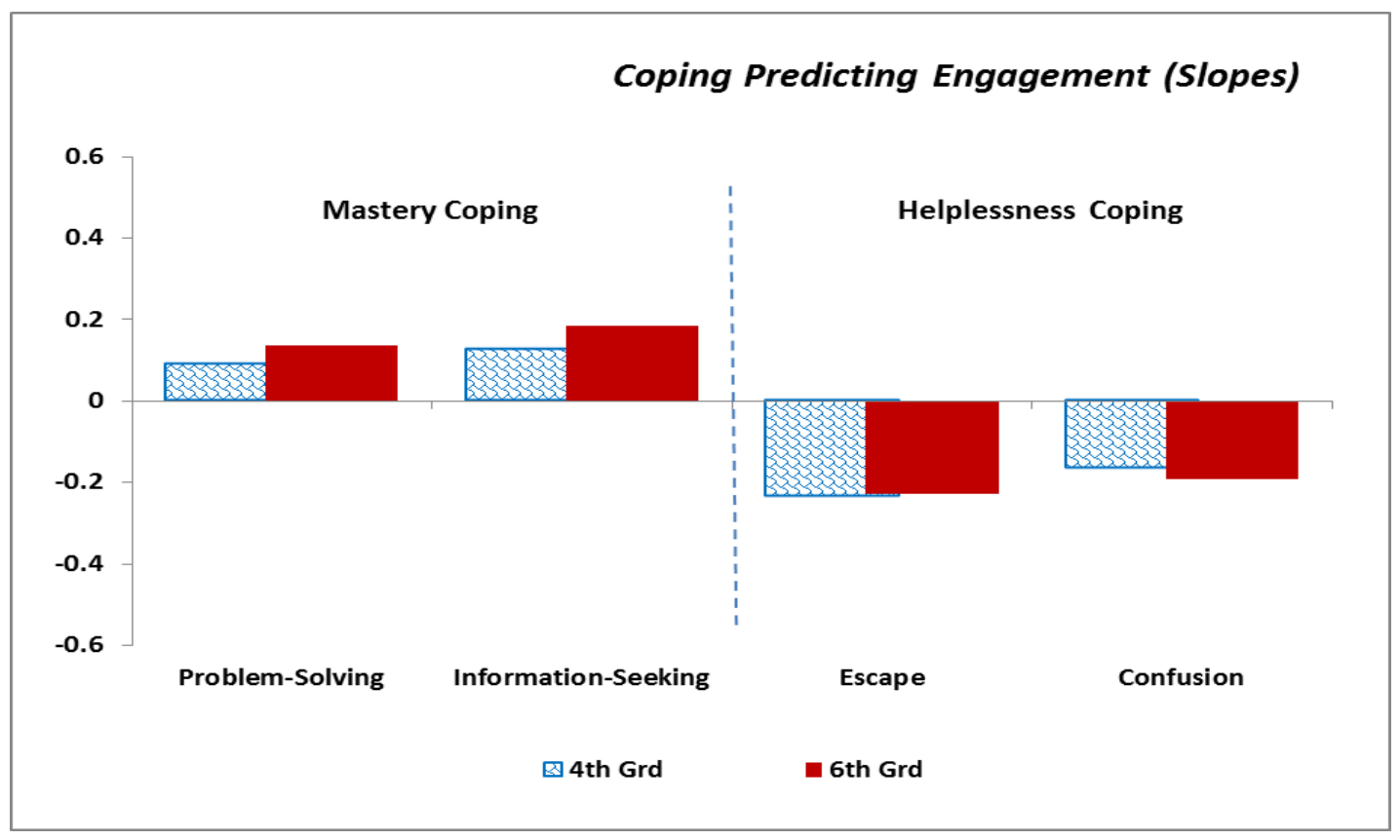

Figure 1.1 Coping predicting Engagement

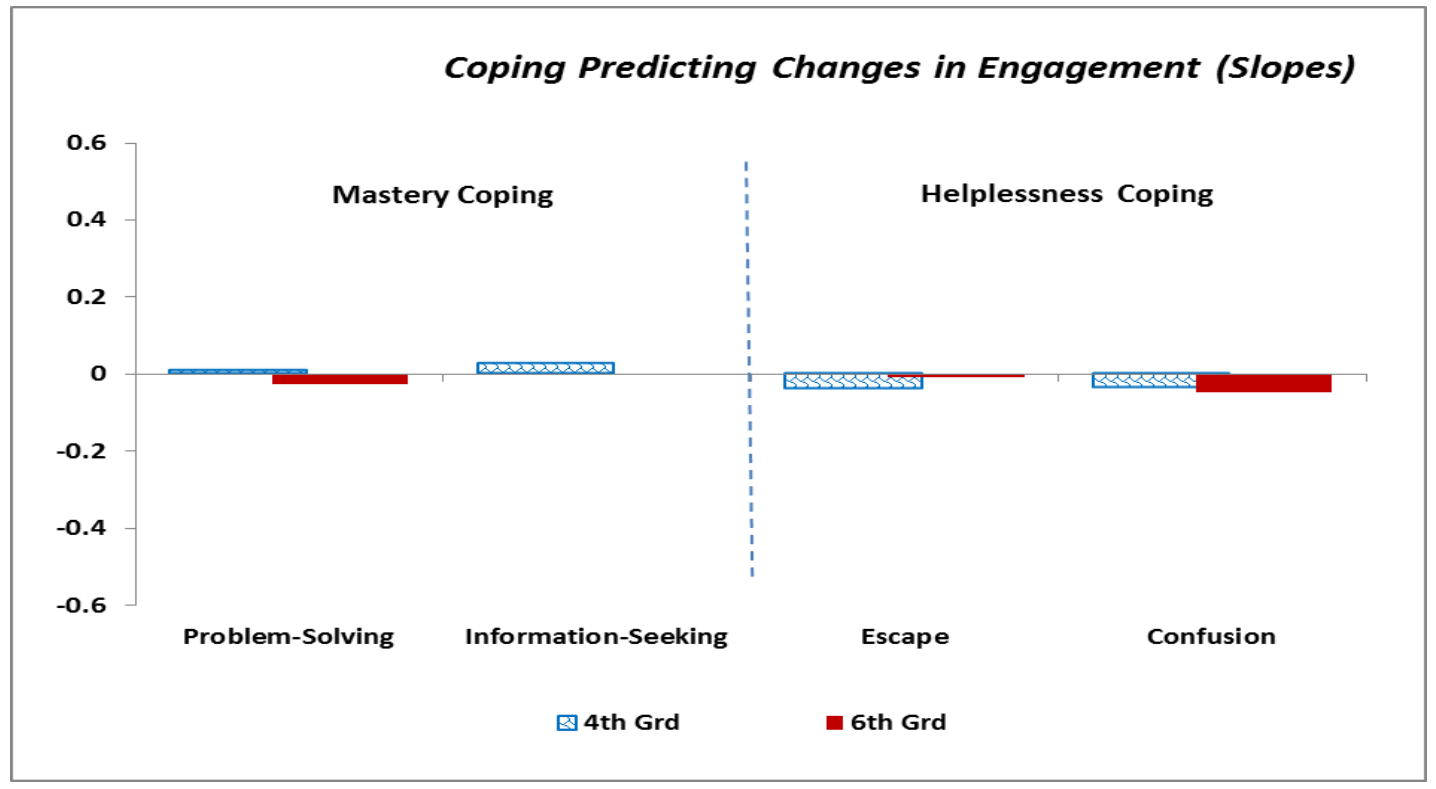

Figure 1.2 Coping predicting Changes in Engagement 
Appendix C 399

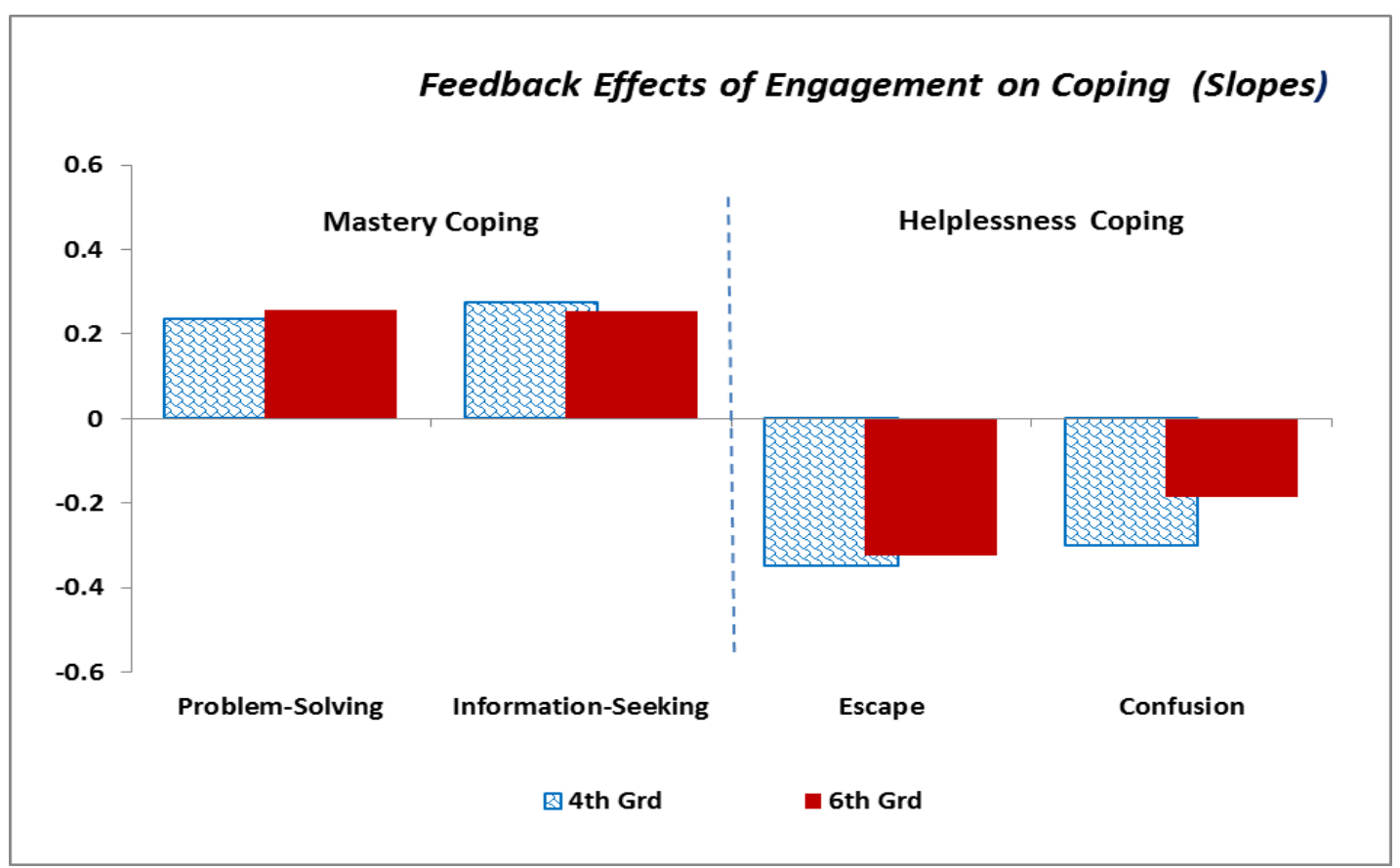

Figure 1.3 Feedback effects of Engagement on Coping

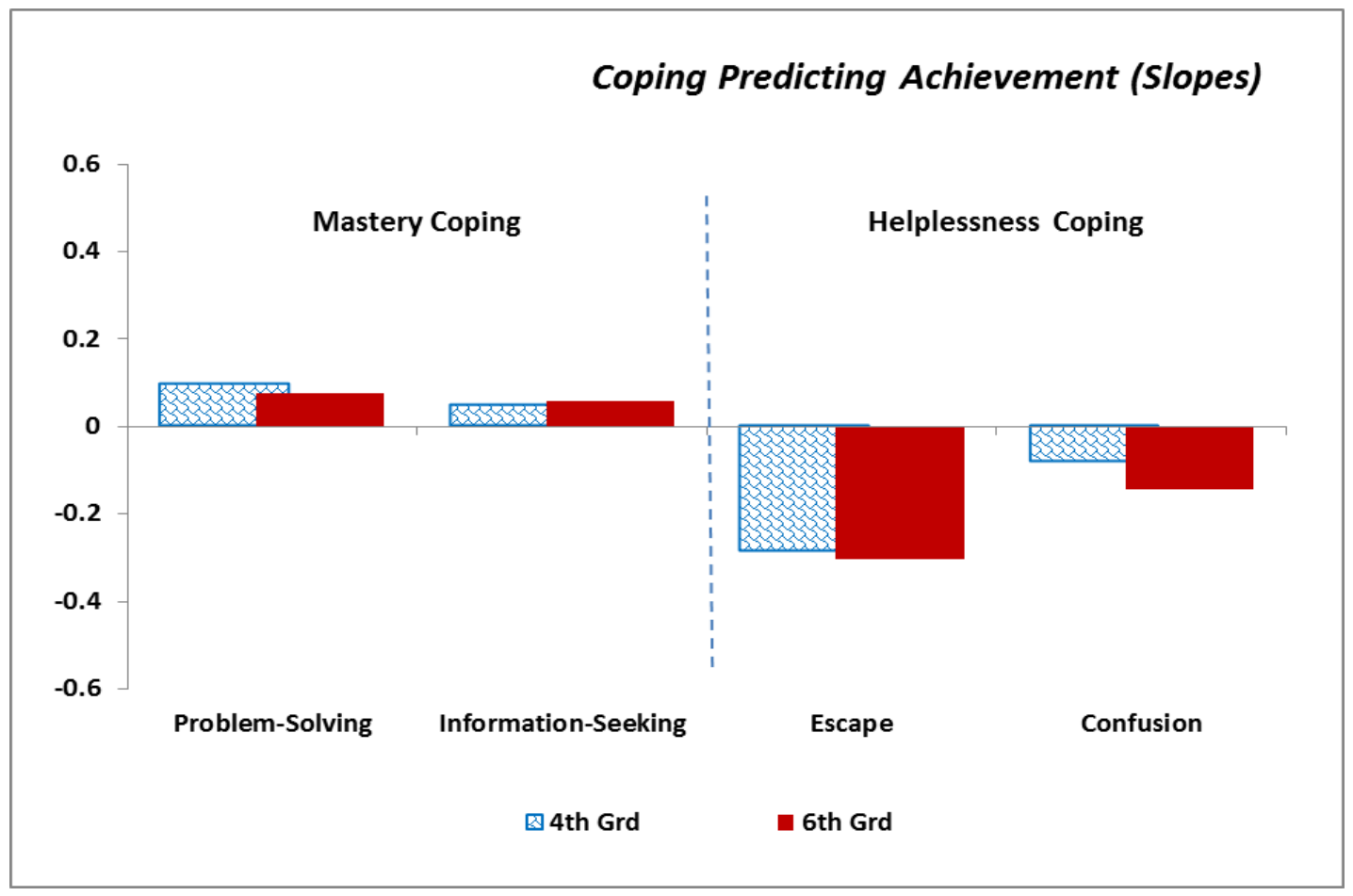

Figure 1.4 Coping predicting Achievement 
Appendix C 400

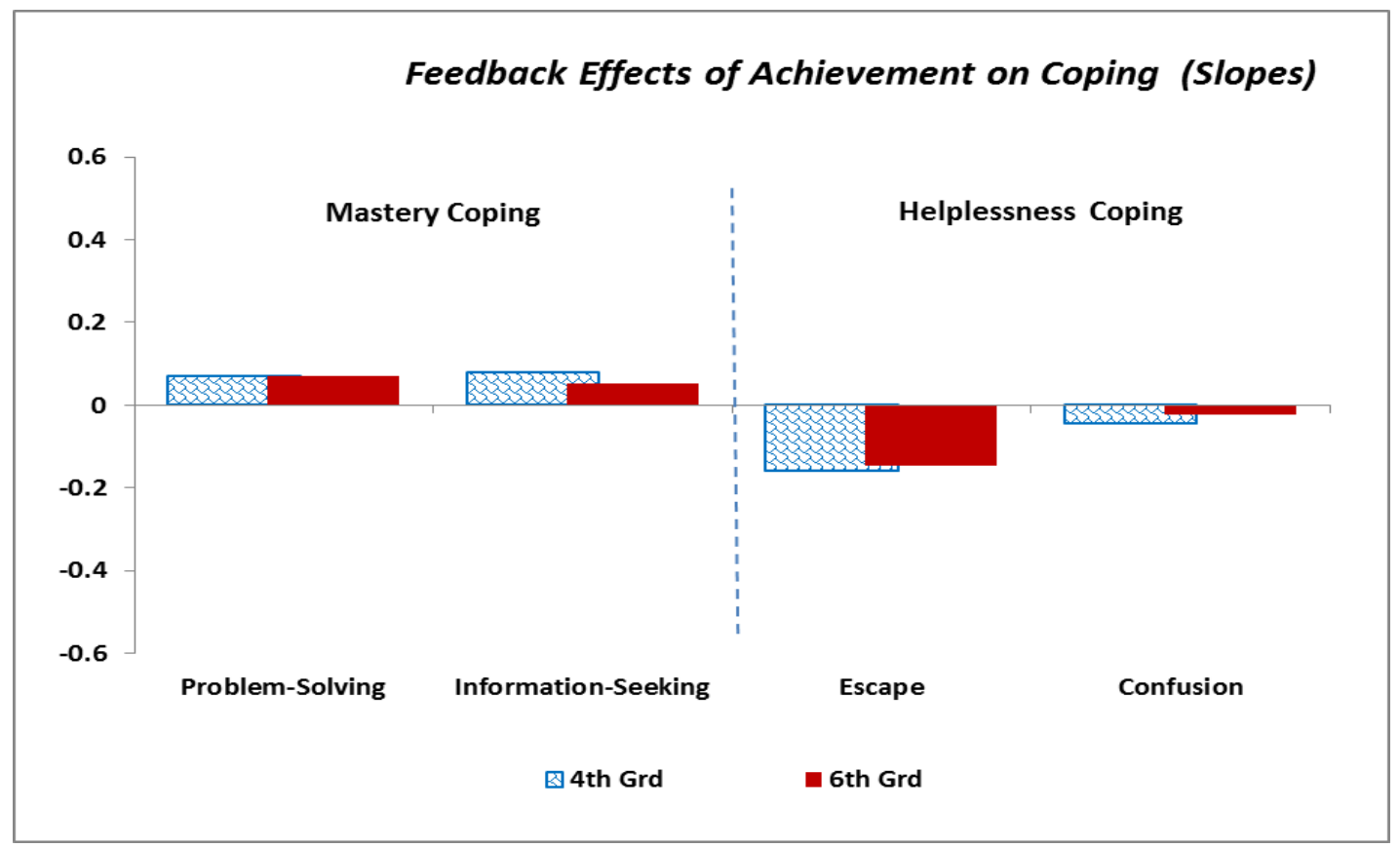

Figure 1.5 Feedback effects of Achievement on coping

Research Question 2: Control - Coping

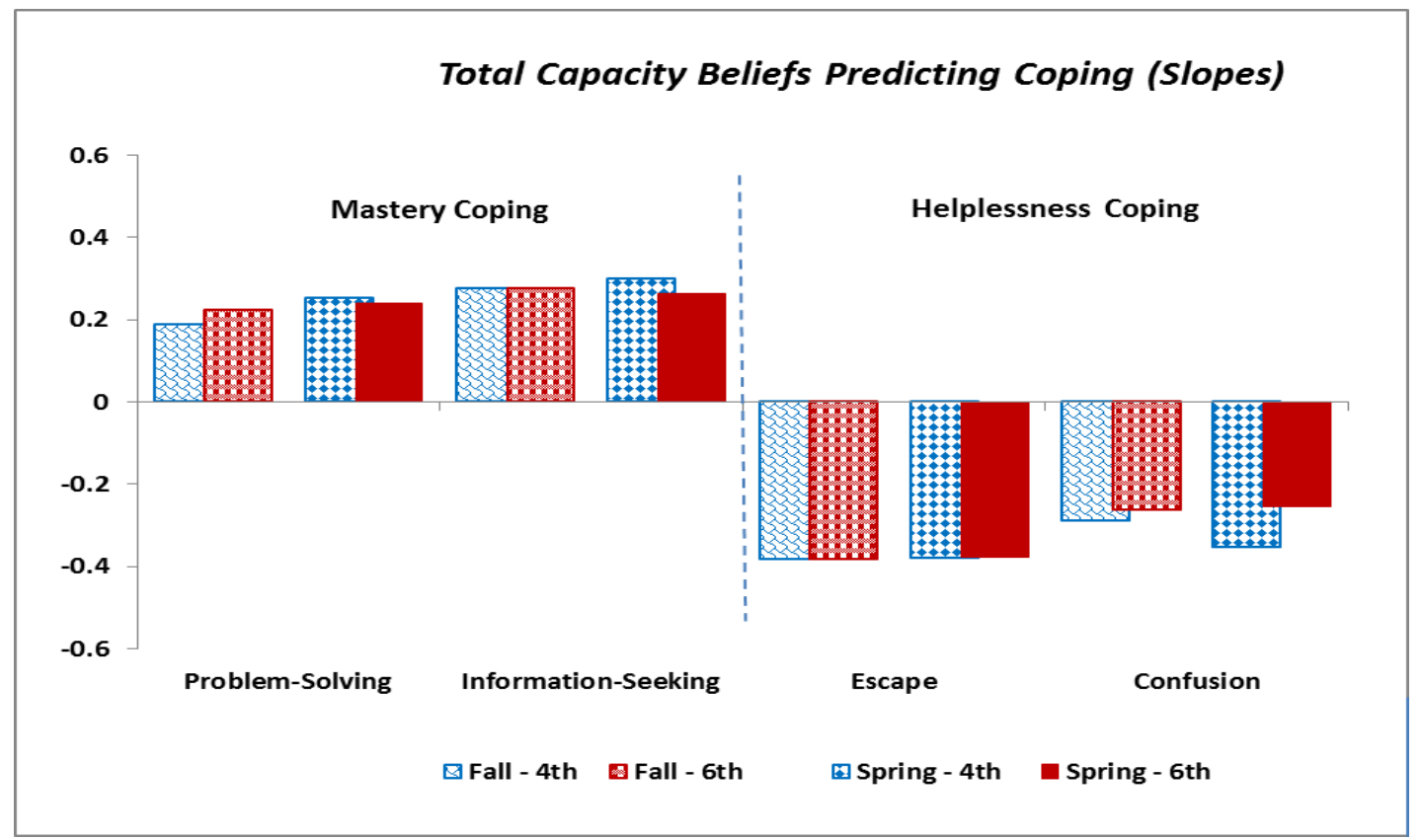

Figure 2.1 Total Capacity Beliefs predicting coping 
Appendix C 401

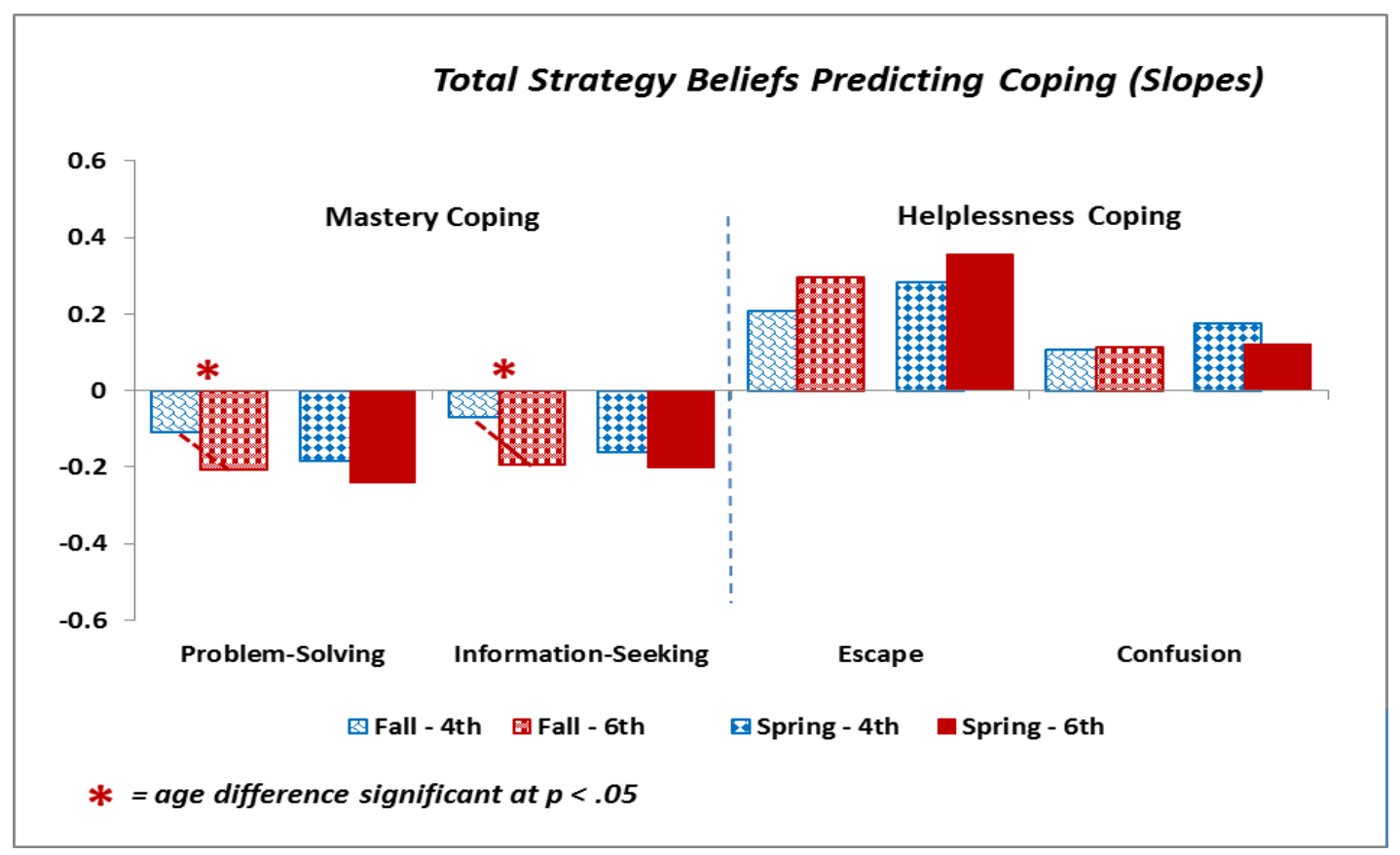

Figure 2.2 Total Strategy Beliefs predicting coping

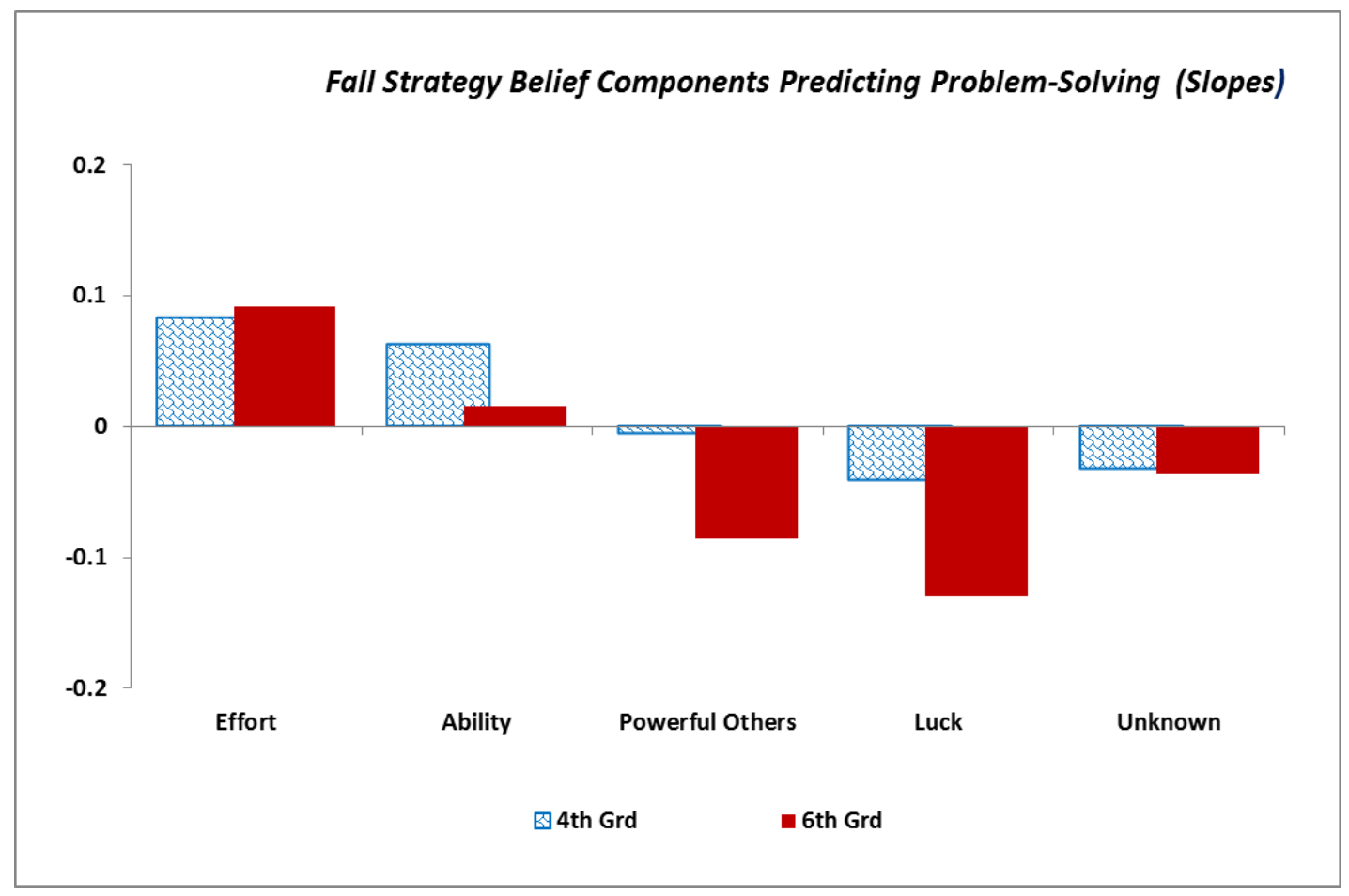

Figure 2.3 Fall strategy belief components predicting Problem-Solving 
Appendix C 402

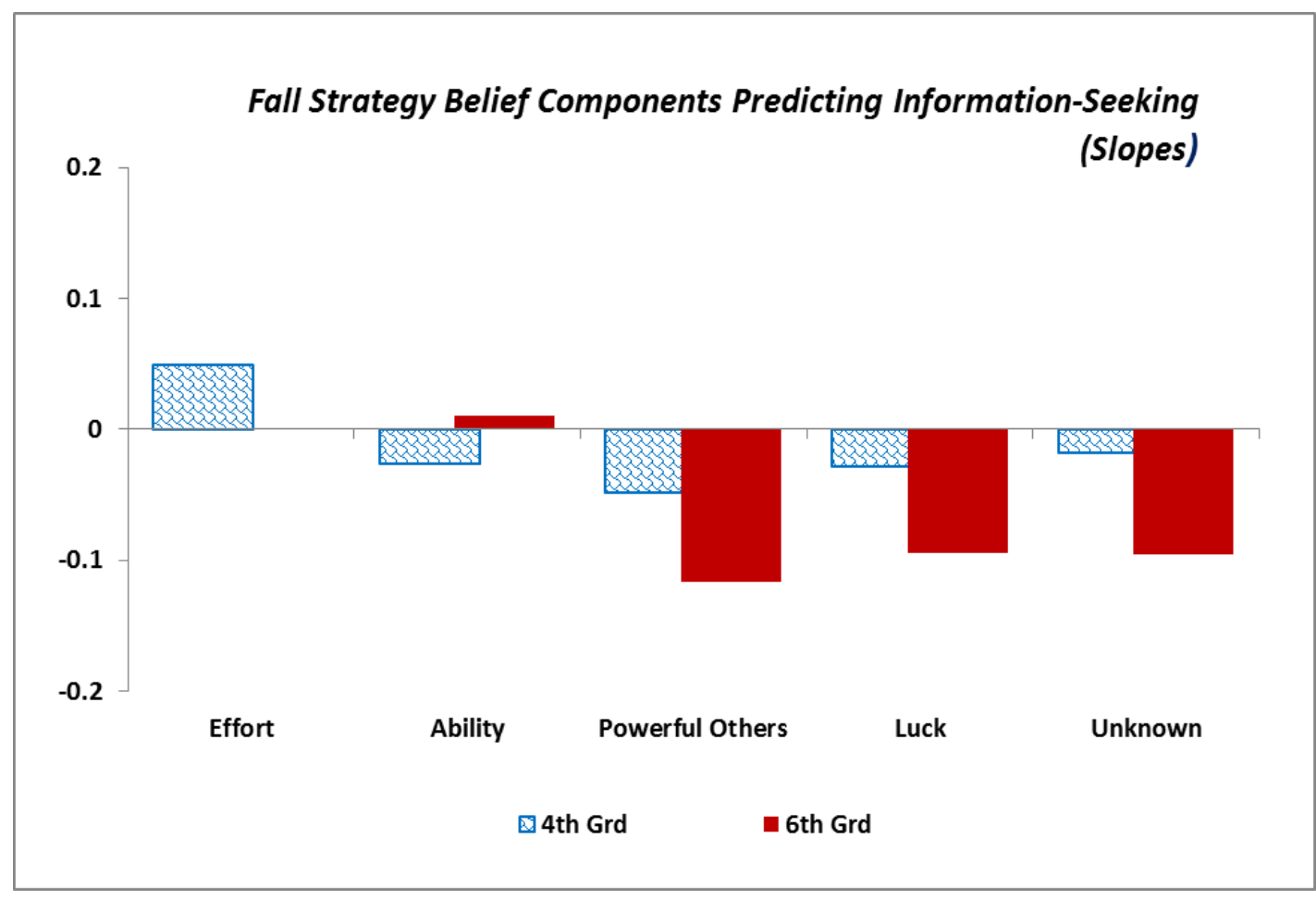

Figure 2.4 Fall strategy belief components predicting Information-Seeking

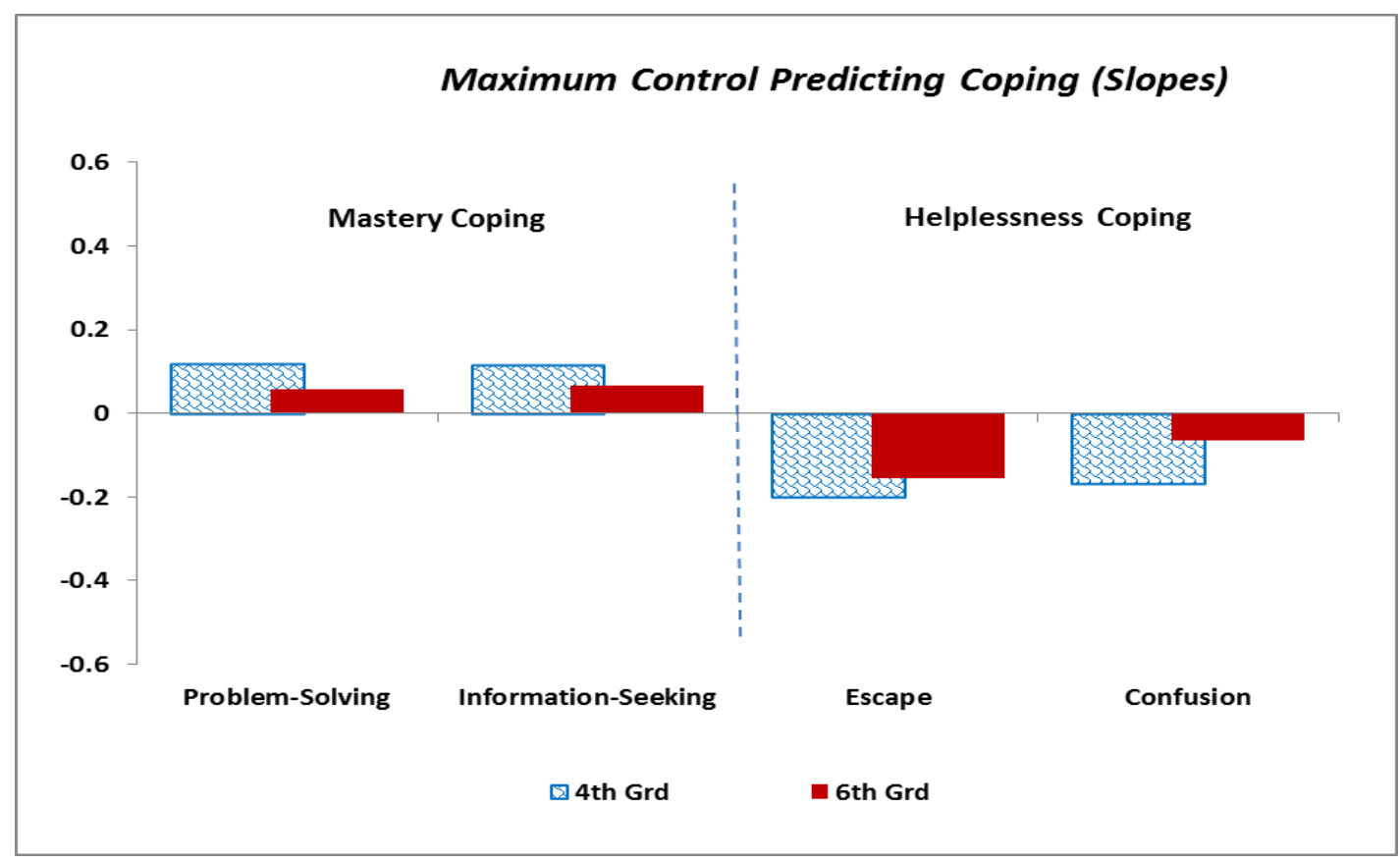

Figure 2.5 Maximum control predicting coping 
Research Question 3: Teacher Support - Coping

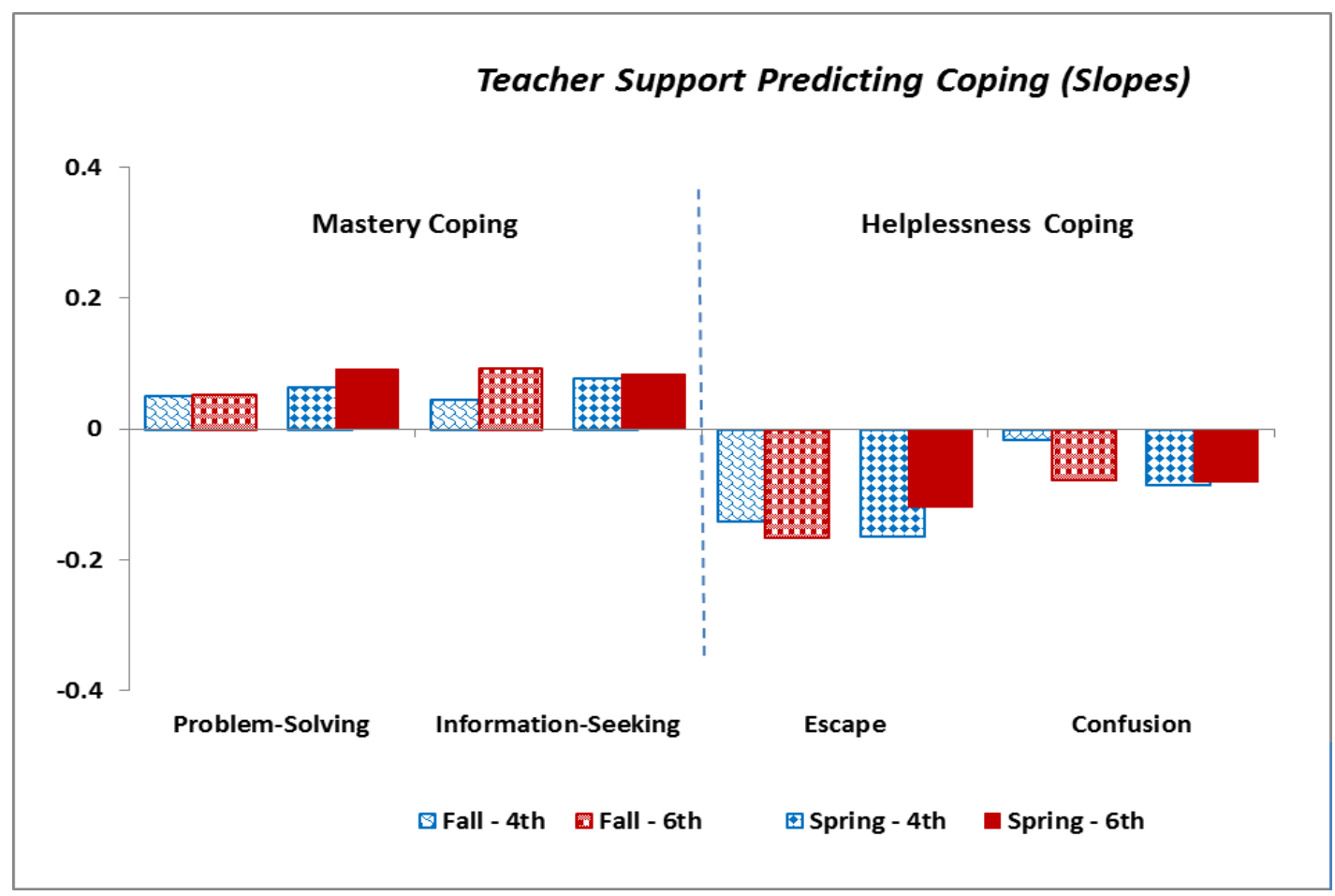

Figure 3.1 Teacher Support predicting coping, concurenntly

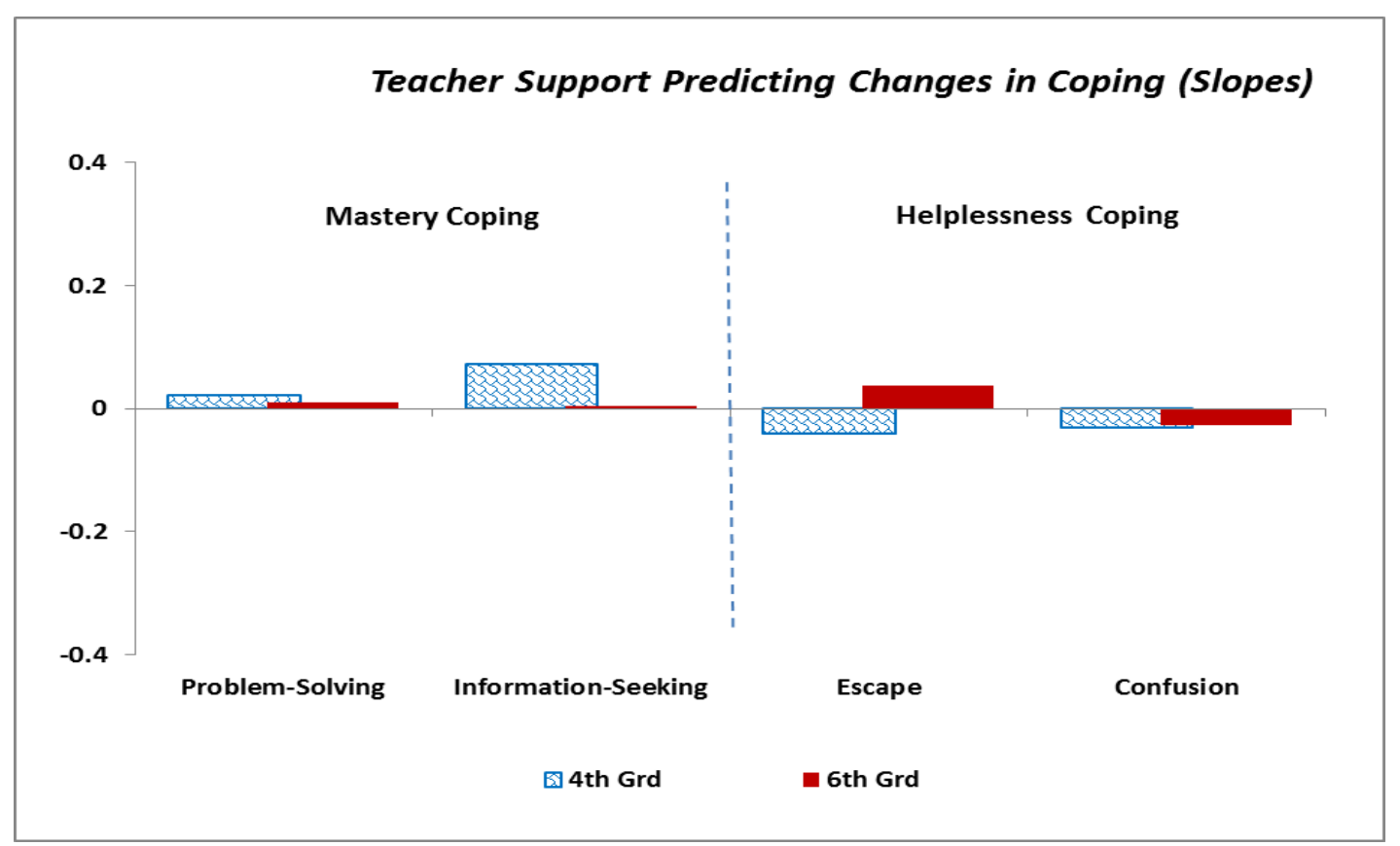

Figure 3.2 Teacher Support predictingchanges in coping 
Research Question 4: Feedback effects of coping and Engagement

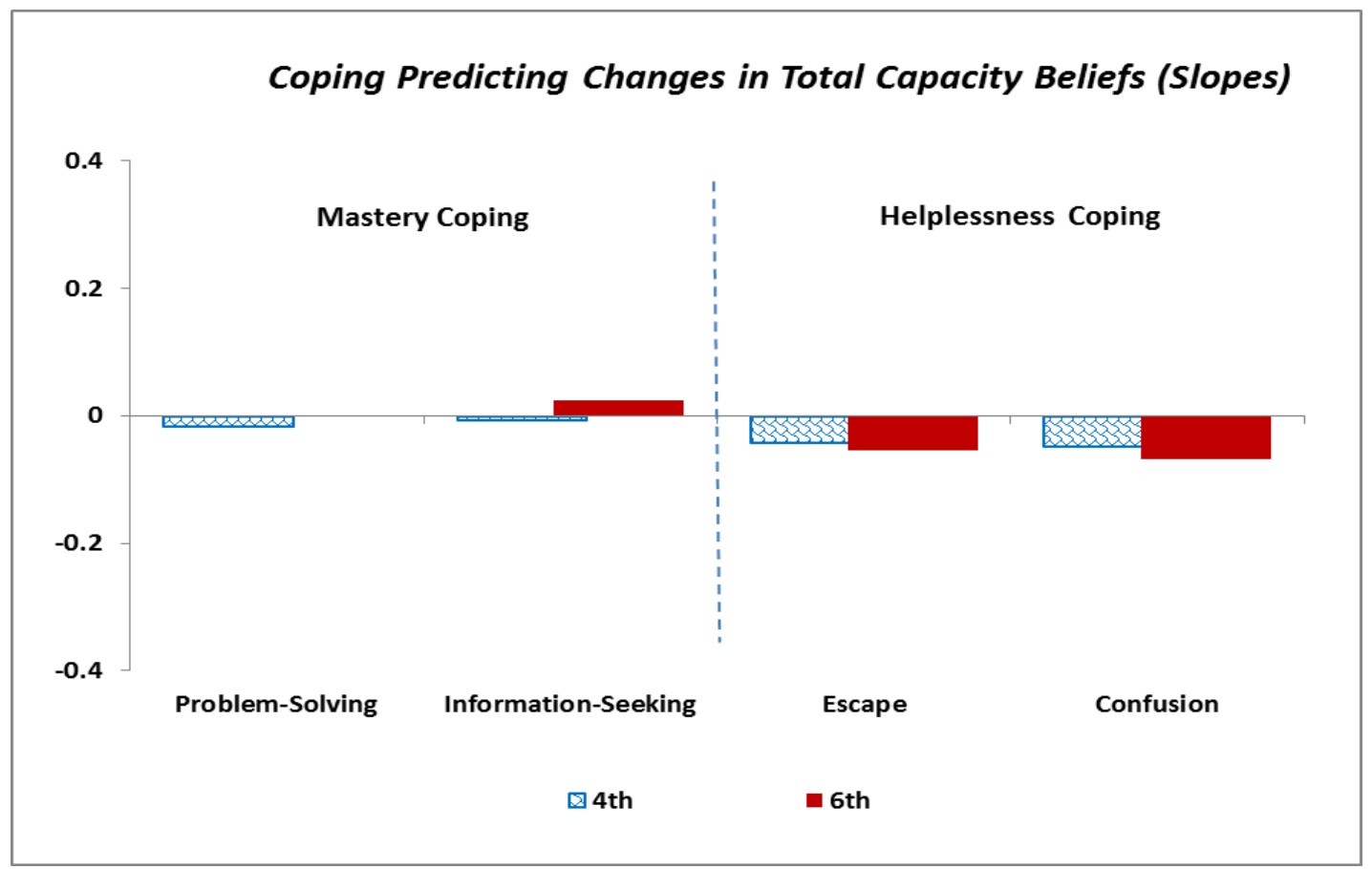

Figure 4.1 Coping predicting changes in Total Capacity Beliefs

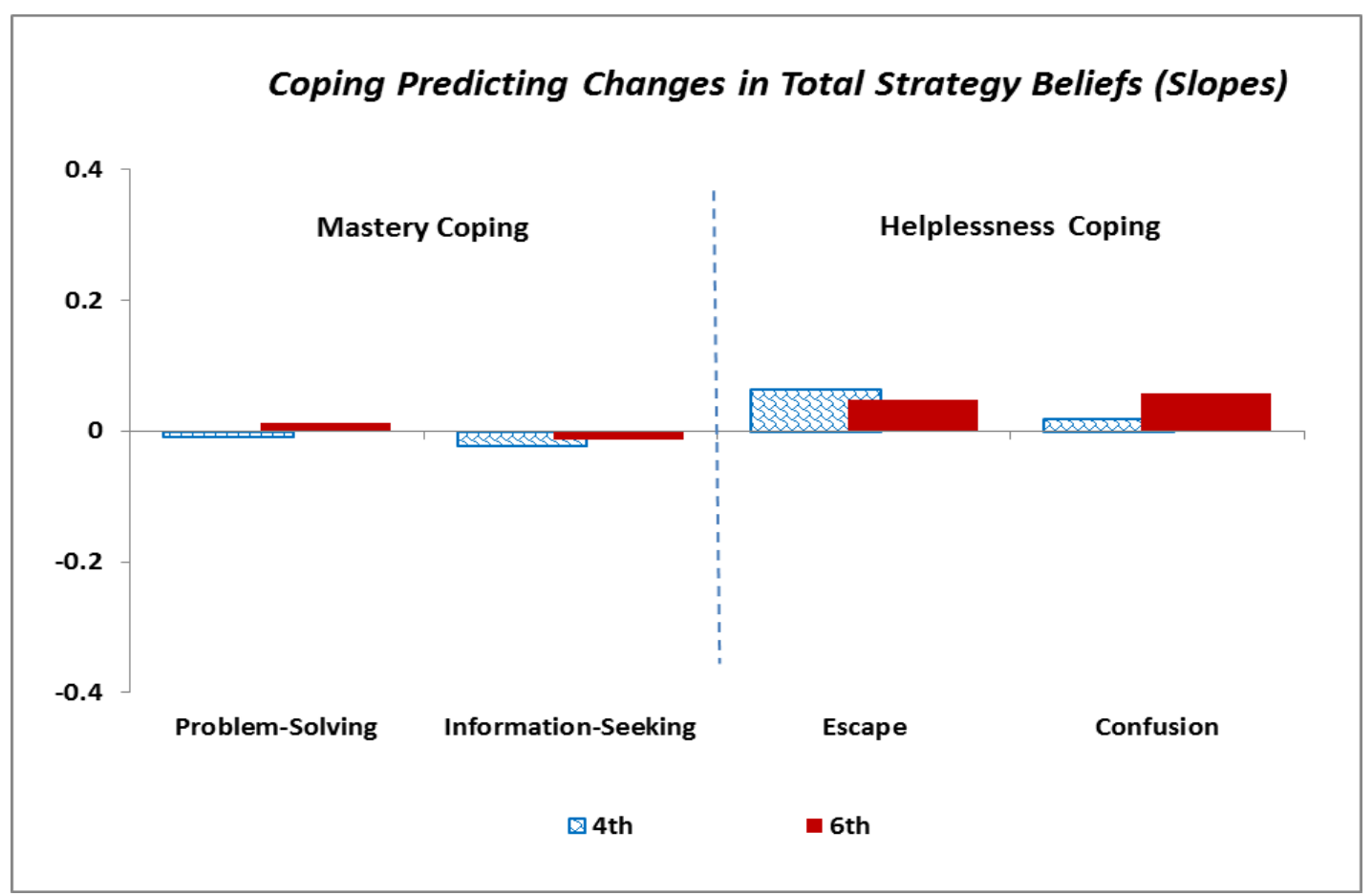

Figure 4.2 Coping predicting changes in Total Strategy Beliefs 


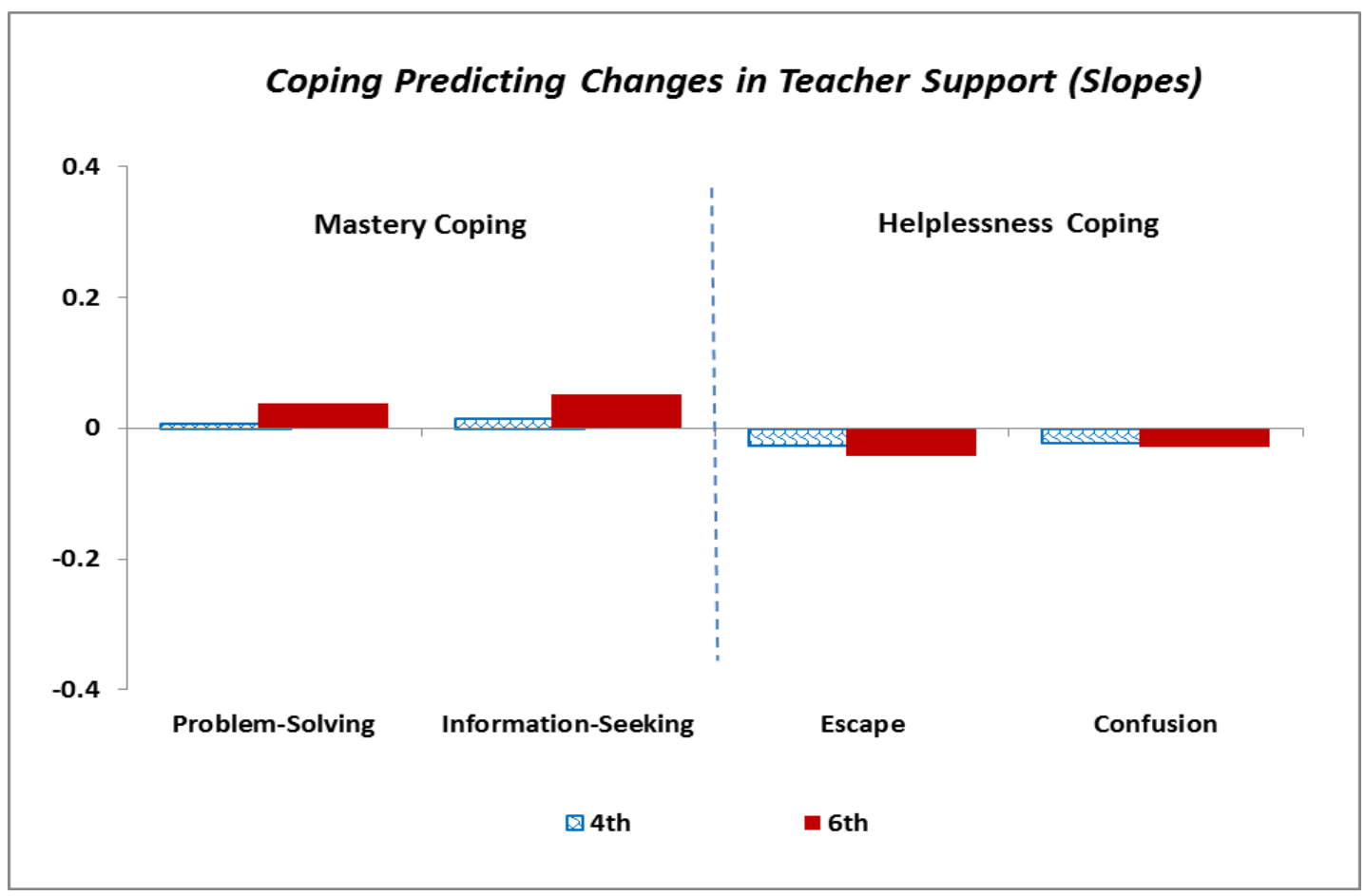

Figure 4.3 Coping predicting changes in Teacher Support

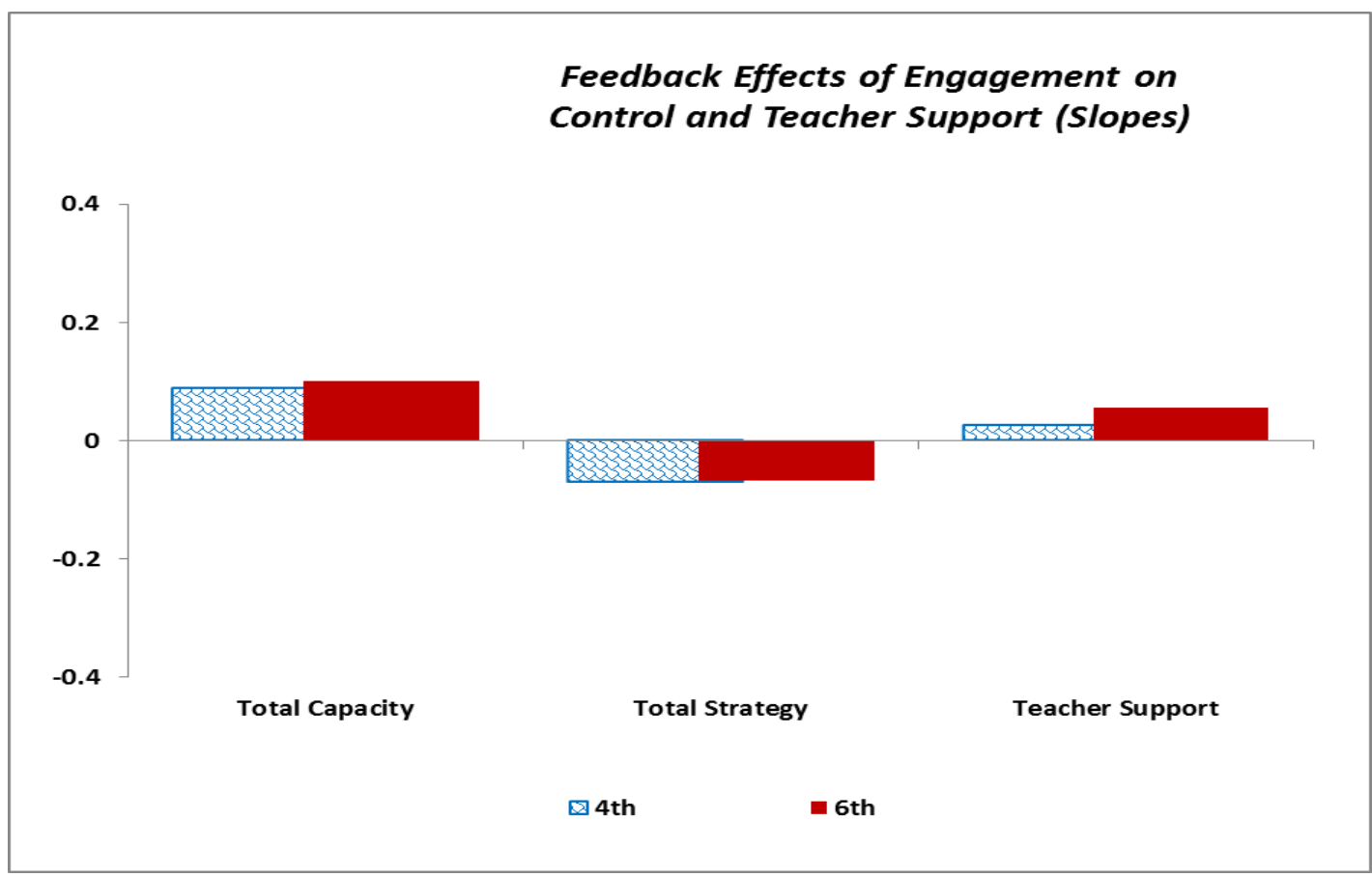

Figure 4.4 Feedback effects of Engagement on control and Teacher Support 